\title{
Automatic and Explicit Parallelization Approaches for Equation Based Mathematical Modeling and Simulation
}

Mahder Gebremedhin 


\title{
Automatic and Explicit Parallelization Approaches for Equation Based Mathematical Modeling and Simulation
}

\author{
Mahder Gebremedhin
}

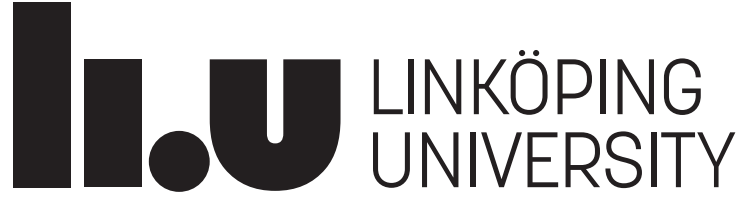

Linköping University

Department of Computer and Information Science

Division for Software and Systems

SE-581 83 Linköping, Sweden

Linköping 2018 


\section{Edition 1:1}

(C) Mahder Gebremedhin, 2018

ISBN 978-91-7685-163-0

ISSN 0345-7524

URL http://urn.kb.se/resolve?urn=urn:nbn:se: liu:diva-152789

Published articles have been reprinted with permission from the respective copyright holder.

Typeset using $\mathrm{X}_{\mathrm{G}} \mathrm{T}_{\mathrm{E}} \mathrm{X}$

Printed by LiU-Tryck, Linköping 2018 


\section{POPULÄRVETENSKAPLIG SAMMANFATTNING}

Övergången från datorer med en processor till datorer med flera processorkärnor ställer krav på att implementera beräkningar på ett sådant sätt att dessa multipla beräkningsenheter kan användas effektivt. Skrivande av effektiva parallella algoritmer är mycket arbetskrävande och en stor källa till fel om inte programmeringsspråk och tillhörande kompilatorer kan förbättras till att erbjuda bättre stödmekanismer. Datorstödd matematisk modellering och simulering är ett av de mest beräkningsintensiva områdena inom datavetenskap. Även simuleringar av förenklade modeller av fysikaliska system kan vara mycket beräkningstungt med användning av standardprocessorer. Att kunna dra nytta av den beräkningskraft som erbjuds av moderna flerkärniga arkitekturer är mycket viktigt inom detta tillämpningsområde. Denna avhandling syftar till att ge bidrag till hur beräkningskraften hos moderna flerkärniga processor kan utnyttja för att öka prestanda för simuleringar, speciellt för modeller uttryckta i det ekvationsbaserade högnivåmodelleringsspråket Modelica, kompilerade och simulerade med användning av OpenModelica's modellkompilator och beräkningsmiljö.

Denna avhandling presenterar två metoder för att simulera matematiska modeller på ett sådant sätt att beräkningskraften hos moderna flerkärniga datorer kan utnyttjas: automatisk respektive explicit parallellisering. Den automatiska metoden utför automatiskt processen att extrahera och använda potentiell parallelism i ekvationssystem från den matematiska modellen utan att programmeraren eller modelleraren behöver göra någon extra ansträngning. I denna avhandling presenteras nya och förbättrade metoder tillsammans med förbättringar i OpenModelicakompilatorn samt ett nytt programbibliotek som stödjer effektiv representation, gruppering, planering, prestandamätning och exekvering av komplexa system av ekvationer och beräkningar, där dessa ofta är beroende av varandra. Den explicita parallelliseringsmetoden utnyttjar parallellism som uttrycks explicit med hjälp av programmeraren eller modelleraren. Nya språkkonstruktioner i Modelicaspråket har introduceras för att göra det möjligt för modellerare att på ett bekvämt sätt uttrycka parallelliserad algoritmer som kan utnyttja beräkningskraften som erbjuds av moderna flerkärniga standardprocessorer och grafikprocessorer. OpenModelicakompilatorn har utökats för att kunna hantera och utnyttja informationen från dessa nya språkkonstruktioner samt att generera parallell kod med ökad beräkningsprestanda. Den genererade koden är portabel till ett antal parallella datorarkitekturer genom OpenCL standarden. Dessutom presenteras prestandamätningar av testmodeller med användning av båda metoderna. 


\begin{abstract}
The move from single-core processor systems to multi-core and manyprocessor systems comes with the requirement of implementing computations in a way that can utilize these multiple computational units efficiently. This task of writing efficient parallel algorithms will not be possible without improving programming languages and compilers to provide the supporting mechanisms. Computer aided mathematical modelling and simulation is one of the most computationally intensive areas of computer science. Even simplified models of physical systems can impose a considerable computational load on the processors at hand. Being able to take advantage of the potential computational power provided by multi-core systems is vital in this area of application. This thesis tries to address how to take advantage of the potential computational power provided by these modern processors in order to improve the performance of simulations, especially for models in the Modelica modelling language compiled and simulated using the OpenModelica compiler and run-time environment.
\end{abstract}

Two approaches of utilizing the computational power provided by modern multi-core architectures for simulation of Mathematical models are presented in this thesis: Automatic and Explicit parallelization respectively. The Automatic approach presents the process of extracting and utilizing potential parallelism from equation systems in an automatic way without any need for extra effort from the modellers/programmers. This thesis explains new and improved methods together with improvements made to the OpenModelica compiler and a new accompanying task systems library for efficient representation, clustering, scheduling, profiling, and executing complex equation/task systems with heavy dependencies. The Explicit parallelization approach allows utilizing parallelism with the help of the modeller or programmer. New programming constructs have been introduced to the Modelica language in order to enable modellers to express parallelized algorithms to take advantage of the computational capabilities provided by modern multicore CPUs and GPUs. The OpenModelica compiler has been improved accordingly to recognize and utilize the information from these new algorithmic constructs and to generate parallel code for enhanced computational performance, portable to a range of parallel architectures through the OpenCL standard.

This work has been supported by Vinnova in the ITEA MODRIO, OPENCPS, and EMPHYSIS projects, and in the Vinnova RTISIM project. Support from the Swedish Government has also been received from the ELLIIT project, as well as from the European Union in the H2020 INTO-CPS project. The OpenModelica development is supported by the Open Source Modelica Consortium. 


\section{ACKNOWLEDGMENTS}

A couple of years ago, in the winter, I missed a bus headed for home. I decided to head back to the university building to avoid the cold. I was roaming the corridors when I came across a Master's thesis project post outside an office that I had seen before but thought it would be too difficult. I decided, since I had nothing to do until the next bus, to go in and ask for more information and see if I can take it. I talked to Peter Fritzson, he asked me a few questions and told me to send him my transcript. A few days later I was working on OpenModelica and just like that here I am finishing up my PhD with Peter after all these years. Since that first day in the winter, Peter has been guiding me and supporting me through all of it. Thank you for giving me the chance and believing in me even when I had my doubts. I would not have started this PhD if it was not for the encouragement from you and I would not have finished it without your support. Thank you for providing a creative and collaborative environment, not just for me, but for all the people working in and around OpenModelica project as well.

PhD life is not easy. It has its fair share of ups and downs, moments where you feel like you are the best at what you do followed by the feeling of being completely lost amid all of it. The research, all the papers, the courses, the supervisions, and all the expectations can be overwhelming at times. I have also had my fair share of missed deliveries and deadlines. Thank you Anne Moe for being patient through all of it. Thank you for all the support, we do really appreciate you. I would also like to thank Eva Pelayo Danils, Åsa Kärrman, Lene Rosell, Inger Norén and all the administration people for making our lives easier.

I would also like to thank all my former and current PELAB colleagues. Christoph Kessler, Kristian Sandahl and everyone else, thank you for providing a collaborative and open working environment. All the bakers for the scrums we had, thank you for the cake, kanelbulle, and cupcakes. Thank you and for all the fika time discussions.

All the OpenModelica developers: Adrian Pop, Arunkumar Palanisamy, Adeel Asghar, Kristain Stavåker, Bernhard Amadeus Thiele, Martin Sjölund, Alachew Shitahun, Lennart Ochel, Per Ostlund, Volker Waurich, Willi Brown, and all others. This work would not have been possible without your constant effort to maintain and improve the OpenModelica ecosystem. I have learned a lot while working with you all and I am thankful for that. Fransceco Casella, thank you for all the pointers and thoughts on the OpenModelica development and for providing some of the libraries used in testing this work.

To all my Ethiopian friends in Linköping, you have been a huge source of encouragement and provided me with outlets to let off some steam from all 
the work. You have provided a home away from home. Thank you for all the Injera and Wot.

My family, if it was not for you, I would not be the man I am today. All the years of education and support has led me to this. I cannot thank you enough. You are my strength and my reliance. We have gone through so much together and I hope this makes most of it worth something.

Last but definitely not least, I would like to thank the Swedish Society as a whole. A civilized, respectful and peaceful society in a civilized country is a rare sight. Perhaps the most important thing I learned over the years I have been here is the Swedish way: tolerance and respect for everyone. It has been a privilege. Thanks for all the fish.

Mahder Gebremedhin

December 2018

Linköping 


\section{Contents}

Abstract

Acknowledgments

Contents $\quad$ ix

List of Figures xiv

List of Tables $\quad$ xvi

$\begin{array}{ll}\text { Listings } & \text { xvii }\end{array}$

1 Introduction $\quad 1$

1.1 Motivation . . . . . . . . . . . . . . . . . . . 1

1.2 Research Problem . . . . . . . . . . . . . . . . . . 3

1.3 Main Contributions ... . . . . . . . . . . . . . 4

1.3.1 Automatic Parallelization .............. 5

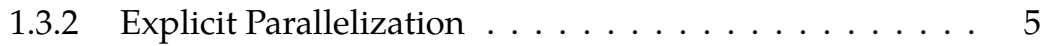

1.4 Practical Considerations . . . . . . . . . . . . . . 6

1.5 Thesis Structure . . . . . . . . . . . . . . . . . . 8

2 Parallel Programming 11

2.1 Introduction . . . . . . . . . . . . . . . . 11

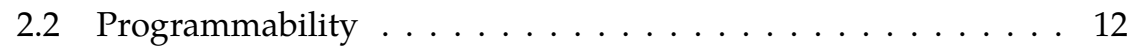

2.2.1 Automatic Parallelization . . . . . . . . . . . . . 12

2.2.2 Explicit Parallelization . . . . . . . . . . . . . . 13

2.3 Memory Model . . . . . . . . . . . . . . . . . . . . . . 13

2.3 .1 Shared Memory . . . . . . . . . . . . . . . 13

2.3.2 Distributed Memory . . . . . . . . . . . . . . . 14

2.4 Threading Model . . . . . . . . . . . . . . . . . . . . . . 15

2.4.1 Data Parallelism . . . . . . . . . . . . . . . 15

2.4 .2 Task Parallelism . . . . . . . . . . . . . . . . . . 16

2.5 Combined Shared Parallelism: Programmability with Threading Model ....................... 16 
3 Mathematical Modeling 19

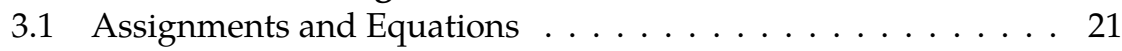

3.1 .1 Notations . . . . . . . . . . . . . . . . 21

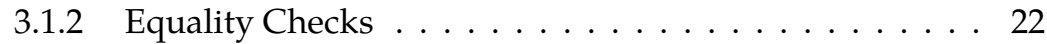

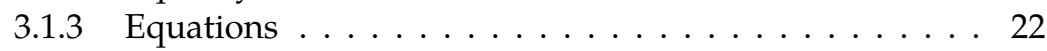

3.1 .4 Inputs and Outputs . . . . . . . . . . . . . 26

3.1 .5 Solving Equation Systems . . . . . . . . . . . . . . 27

3.2 Dynamic Systems: Time . . . . . . . . . . . . . . . . . . . . . . 28

3.3 Rate Of Change: Derivatives . . . . . . . . . . . . . . . . . . . . . . . . . . . . . 39

3.4 Discrete Behaviour . . . . . . . . . . . . . . . . . . 32

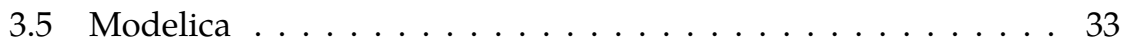

3.5.1 Modelica for Mathematical Modeling . . . . . . . . . . . . . 33

3.6 Modelica Standard Library (MSL) . . . . . . . . . . . . . . . . . . . . . 36

3.7 OpenModelica . . . . . . . . . . . . . . . 36

I Automatic Parallelization $\quad 39$

4 Introduction $\mathbf{4 1}$

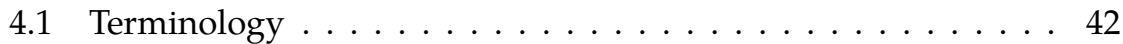

4.1 .1 Graphs ..................... 42

4.1 .2 Directed Graphs . . . . . . . . . . . . . . . . . . . 43

4.1 .3 Bipartite Graphs . . . . . . . . . . . . . . . . . . . 44

4.2 Causalization of Equation Systems . . . . . . . . . . . . . . . . . . . . . . . 44

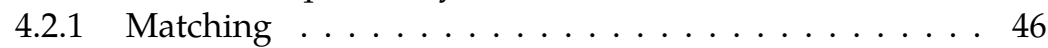

4.2 .2 Sorting .................... 47

4.3 Automatic Parallelization Approaches . . . . . . . . . . . . . . . . . . . . 49

4.3.1 Parallelization Over Method . . . . . . . . . . . . . . . . . . . 49

4.3.2 Parallelization Over Time . . . . . . . . . . . . . . 51

4.3.3 Parallelization Over Equation System . . . . . . . . . . . . 51

5 Connected Component Parallelization $\mathbf{5 5}$

5.1 Integrated Approach . . . . . . . . . . . . . . . . 56

5.2 Cost Estimation and Load Balancing . . . . . . . . . . . . . . . . . 56

5.3 Memory Management . . . . . . . . . . . . . . . . . . 58

5.3 .1 Shared Global Memory Pool . . . . . . . . . . . . . . 59

5.3 .2 Thread Local Memory Pool . . . . . . . . . . . . . . . . . 60

5.4 Thread Management . . . . . . . . . . . . . . . . 61

5.4 .1 Complexity and Portability Issues . . . . . . . . . . . 62

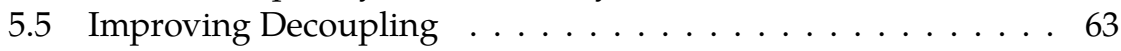

5.6 Case Study . . . . . . . . . . . . . . . . . . . . . . . . . . . . . . . . . 66

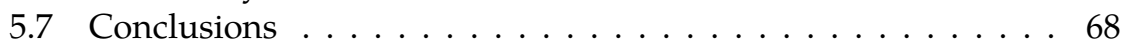

6 Strongly Connected Components Parallelization 71 
6.1 Equation System Structure $\ldots \ldots \ldots \ldots$. . . . . . . . . . 72

6.2 The Need for Scheduling . . . . . . . . . . . . . . 73

6.3 Data Dependencies . . . . . . . . . . . . . . . . . . . . 74

6.4 The Need for Clustering . . . . . . . . . . . . . . . . . 76

6.5 Stand Alone Implementation . . . . . . . . . . . . . . . 76

6.6 Memory and Thread Management . . . . . . . . . . . 77

7 Clustering and Scheduling $\quad 81$

7.1 Task Clustering: Reducing Overhead, Improving Locality, and

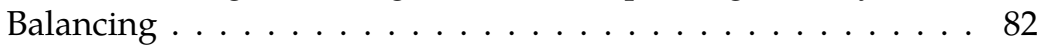

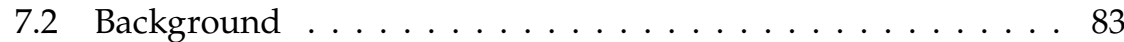

7.2.1 The Bin Packing Problem . . . . . . . . . . . . 83

7.2.1.1 Polynomial Time Bin Packing Approximations 84

$7.2 .2 k$-way Integer Partitioning . . . . . . . . . . . . . . 86

7.2.3 Makespan Scheduling Approximation Algorithms . . . 87

7.3 Clustering Heuristics . . . . . . . . . . . . . . . . . . . 88

7.3.1 Merge Single Parent (MSP) _ . . . . . . . . . . . . 88

7.3.2 Merge Level Parents (MLP) . . . . . . . . . . . . . . 89

7.3.3 Merge Level for Bins (MLB) . . . . . . . . . . . . . . . 90

7.3.4 Merge Level for Cost (MLC) . . . . . . . . . . . . . 92

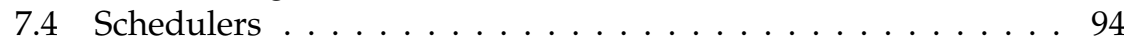

7.4.1 The Level Scheduler . . . . . . . . . . . . . . . . . . . 95

7.4 .2 Flow Graph Scheduler . . . . . . . . . . . . . . . 98

8 ParModAuto $\quad 99$

8.1 Motivation . . . . . . . . . . . . . . . . . . . 99

8.2 Design Principles . . . . . . . . . . . . . . . . . . 100

8.2.1 High Level Operation . . . . . . . . . . . . . . . . . 100

8.2 .2 Runtime Processing . . . . . . . . . . . . . . . . . 100

8.2.3 Portability Considerations . . . . . . . . . . . . . . 101

8.2.4 Extensibility Considerations . . . . . . . . . . . . 102

8.2.5 Independence: Minimal Assumptions . . . . . . . . . 102

8.3 Implementation . . . . . . . . . . . . . . . . . . 103

8.3.1 Task Abstraction . . . . . . . . . . . . . . . . . . . . 103

8.3.2 Clusters . . . . . . . . . . . . . . . . . . . . . 104

8.3.3 Dependency Specification and Task System Construction105

8.3.4 Task System Representation . . . . . . . . . . . . . . . . . 108

8.3 .5 Schedulers . . . . . . . . . . . . . . . . . . . . 109

8.3.6 Equation System Representation . . . . . . . . . . . . 110

8.3.7 Extra Functionalities . . . . . . . . . . . . . . . . . 112

9 Performance Evaluation 113

9.1 Overview . . . . . . . . . . . . . . . . . . . . 113

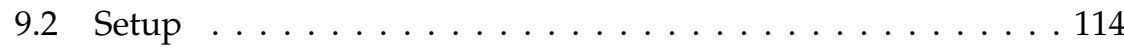


9.3 Test Cases and Results . . . . . . . . . . . . . . . . . . . . . . 115

9.3.1 CauerLowPassSC . . . . . . . . . . . . . . 116

9.3.2 BranchingDynamicPipes . . . . . . . . . . . . . 117

9.3.3 Spice3BenchmarkFourBitBinaryAdder . . . . . . . . . 118

9.3 .4 EngineV6 . . . . . . . . . . . . . . . . . . . . . . . 119

9.3 .5 SteamPipe . . . . . . . . . . . . . . . . . 121

10 Conclusions on Automatic Parallelization 127

II Explicit Parallelization $\quad 129$

11 Introduction 131

11.1 General Purpose Graphic Processing Unit (GPGPU) program-

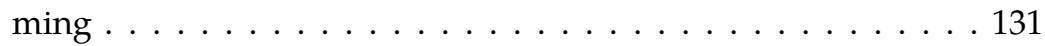

11.2 OpenCL . . . . . . . . . . . . . . . . . . . 132

11.2.1 The OpenCL Architecture . . . . . . . . . . . . . . 132

11.2.2 Platform Model . . . . . . . . . . . . . . . . . . . 132

11.2.3 Execution Model . . . . . . . . . . . . . . . . . . . . . . 132

11.2.4 Memory Model . . . . . . . . . . . . . . . . . . . 133

11.2.5 Programming Model . . . . . . . . . . . . . . . . . 134

11.3 Modelica for Scientific Computing . . . . . . . . . . . . . 135

11.4 Related work . . . . . . . . . . . . . . . . . . . 138

12 ParModelica Extensions 141

12.1 Parallel Variables . . . . . . . . . . . . . . . . . . . . . . . . 143

12.2 Parallel Functions . . . . . . . . . . . . . . . . . . . . . 144

12.3 Kernel Functions . . . . . . . . . . . . . . . . . . . . . 145

12.4 Parallel For Loop: parfor . . . . . . . . . . . . . . . . . . 146

12.5 Built-in Functions . . . . . . . . . . . . . . . . . . . . . . 147

12.5.1 Synchronization and Thread Management . . . . . . . . 148

12.5.2 Extra OpenCL Functionalities . . . . . . . . . . . . . . . 149

13 ParModExp 153

13.1 ParModelica OpenCL-C Runtime Library . . . . . . . . . . . . . 153

13.2 ParModelica OpenCL Utility Headers . . . . . . . . . . . . . 154

14 Performance Evaluations 157

14.1 The MPAR Benchmark Suite . . . . . . . . . . . . . . . . . 157

14.1.1 Results . . . . . . . . . . . . . . . . . 158 
III Appendix 163

15 Numerical Methods 165

15.1 Non-Linear Systems: Root Finding and Newton's method . . . . 165

15.2 Numerical Integration . . . . . . . . . . . . . . . . . . 167

15.2.1 Euler's Methods . . . . . . . . . . . . . . . . . . . 168

15.2.2 Adam-Bashforth Methods . . . . . . . . . . . . . . . . . 169

15.2.3 Adam-Moulton Methods . . . . . . . . . . . . . . 170

15.2.4 Backward Differentiation Formulae (BDF) Methods . . . 172

15.2.5 DASSL . . . . . . . . . . . . . . . . . . . . . . . . 173

16 ParModelica (Extended Modelica) Concrete Syntax 175

16.1 Lexical conventions . . . . . . . . . . . . . . . . . . . . . 175

16.2 Grammar . . . . . . . . . . . . . . . . . . . . . 177

16.2.1 Stored Definition - Within . . . . . . . . . . . . 177

16.2.2 Class Definition . . . . . . . . . . . . . . . . . . . 177

16.2.3 Extends . . . . . . . . . . . . . . . . . . . . . . 179

16.2.4 Component Clause . . . . . . . . . . . . . . . . . 179

16.2 .5 Modification . . . . . . . . . . . . . . . . . . 179

16.2.6 Equations . . . . . . . . . . . . . . . . 180

16.2 .7 Expressions . . . . . . . . . . . . . . . . . 182

17 Selected ParModExp OpenCL Library API definitions 185

$\begin{array}{ll}\text { Bibliography } & 187\end{array}$ 


\section{List of Figures}

3.1 A simple pendulum . . . . . . . . . . . . . . . . . 34

3.2 OpenModelica compiler's compilation phases . . . . . . . 37

4.1 A simple electrical circuit . . . . . . . . . . . . . . . 46

4.2 Bipartite graphs RLC circuit equations . . . . . . . . . . . 48

4.3 Directed Graph of matched RLC circuit equations . . . . . . 48

4.4 Parallelization Opportunities . . . . . . . . . . . . . . . . 50

5.1 Simple Connected Component Balancing . . . . . . . . . . . 56

5.2 Simplified thread guidance through runtime system. . . . . . 63

5.3 Delayed variable dependencies . . . . . . . . . . . . . 65

5.4 Delayed variable solution trajectories . . . . . . . . . . . . 66

5.5 A volume with a pressure relief valve . . . . . . . . 67

5.6 Speed-up for different number of segments . . . . . . . . . 68

6.1 A simple electrical circuit . . . . . . . . . . . . . . . . 72

6.2 Directed Graph of matched RLC circuit equations . . . . . . 74

7.1 Merge Single Parent . . . . . . . . . . . . . . . . . . 88

7.3 Merge Level Parents . . . . . . . . . . . . . . . . . . . . . . . 89

7.5 Cycles in parent merging . . . . . . . . . . . . . . . 91

7.6 FourBitBinaryAdder model equation structure after symbolic processing . . . . . . . . . . . . . . . . 94

7.7 FourBitBinaryAdder model equation after applying MSP, MLP and MLB . . . . . . . . . . . . . . . . . . . . . . . . 95

7.8 Level Scheduler operation flow. . . . . . . . . . . . . . . 97

9.1 Speed-up for CauerLowPassSC model . . . . . . . . . . . . 117

9.2 Speed-up for BranchingDynamicPipes model . . . . . . . . . 118

9.3 Speed-up for Spice3BenchmarkFourBitBinaryAdder model . . 119

9.4 Speed-up for EngineV6 model . . . . . . . . . . . . . . . . 120

9.5 Speed-up for SteamPipe320 model . . . . . . . . . . . . . . . . 122

9.6 Speed-up for SteamPipe640 model . . . . . . . . . . . . . 123 
9.7 Speed-up for SteamPipe1280 model . . . . . . . . . . . . . 124

9.8 Speed-up for SteamPipe2560 model . . . . . . . . . . . . 125

11.1 OpenCL Memory Model. . . . . . . . . . . . . . . . . . . . . 134

12.1 Pre-ParModelica vs ParModelica parallel programming in Modelica . . . . . . . . . . . . . . . . . . . . . . . . . . . 142

14.1 Speedups for matrix multiplication . . . . . . . . . . . . 158

14.2 Speedups for Eigenvalue computations . . . . . . . . . . . 159

14.3 Equidistant computation grid . . . . . . . . . . . . . . . . 159

14.4 Speedups for $2 \mathrm{~d}$ heat plate computations . . . . . . . . 160

15.1 Euler's Methods . . . . . . . . . . . . . . . . . . . . . . . . . 168

$15.43^{\text {rd }}$ order Adam-Bashforth $\ldots . . . \ldots 169$

$15.63^{\text {rd }}$ order Adam-Moulton . . . . . . . . . . . . . . . 171

$15.82^{\text {nd }}$ order Backward Differentiation Formulae $\ldots \ldots \ldots \ldots$ 


\section{List of Tables}

2.1 Supported Parallelism . . . . . . . . . . . . . . . . 17

9.1 Summary of the CauerLowPassSC model . . . . . . . . . . . 117

9.2 Summary of the BranchingDynamicPipes model . . . . . . . . 118

9.3 Summary of the Spice3BenchmarkFourBitBinaryAdder model . . . 119

9.4 Summary of the EngineV6 model . . . . . . . . . . . . . . 120

9.5 Summary of the SteamPipe320 model . . . . . . . . . . . . 122

9.6 Summary of the SteamPipe640 model . . . . . . . . . . . . . . 123

9.7 Summary of the SteamPipe1280 model . . . . . . . . . . . . . . 124

9.8 Summary of the SteamPipe2560 model . . . . . . . . . . . . 125

11.1 OpenCL allocation and memory access capabilities . . . . . . . 134

12.1 Parallel Variable Assignment Operation . . . . . . . . . . . . 143 


\section{Listings}

3.1 Algorithm for Equation $3.3 \ldots \ldots \ldots \ldots$

3.2 Equation-based program for Equation $3.10 \ldots \ldots \ldots$

3.3 A simple simulation . . . . . . . . . . . . . . . . . . . . . . . 29

3.4 Second Law . . . . . . . . . . . . . . . . 30

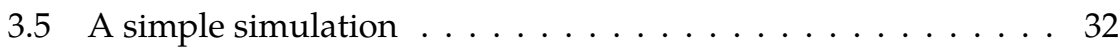

3.6 A Modelica pendulum model . . . . . . . . . . . . . . 35

5.1 Shared global pool allocations . . . . . . . . . . . . . . . 59

5.2 Delays in Modelica model . . . . . . . . . . . . . . . . . . 64

7.1 Next Fit Algorithm . . . . . . . . . . . . . . . . 85

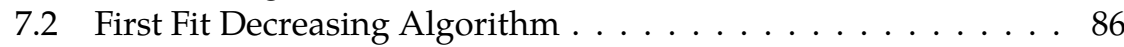

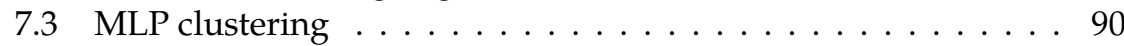

7.4 Merge Level for Bins . . . . . . . . . . . . . . . . . 92

7.5 Merge Level for Cost . . . . . . . . . . . . . . . . . . . 93

8.1 Simplified Equation Task . . . . . . . . . . . . . . . . . . 103

8.2 execute Function definition for Equation functions . . . . . . . 103

8.3 A ParModAuto Cluster Class . . . . . . . . . . . . . . . . . . . 104

8.4 Dependency JSON file . . . . . . . . . . . . . . . . . . . . . . 105

8.5 A ParModAuto TaskSystem Class . . . . . . . . . . . . . . . 108

8.6 The ParModelica Level Scheduler class . . . . . . . . . . . . 110

8.7 A Model class representing an OpenModelic model . . . . . . 110

11.1 Unknow size arrays . . . . . . . . . . . . . . . . 135

11.2 Array slicing . . . . . . . . . . . . . . . . . 136

11.3 Reduction operations . . . . . . . . . . . . . . . . 138

12.1 ParModelica vs MATLAB GPU variables . . . . . . . . . 142

12.2 ParModelica device variables . . . . . . . . . . . . . . . 143

12.3 ParModelica parallel functions . . . . . . . . . . . . . . . . 144

12.4 ParModelica kernel functions . . . . . . . . . . . . . 145

12.5 ParModelica parallel for loops . . . . . . . . . . . . . . . 146

12.6 Loading and executing external OpenCL kernels . . . . . . 150

13.1 ParModelica device array . . . . . . . . . . . . . . . . 153 



\section{Introduction}

\subsection{Motivation}

Build faster processors. This used to be the way to get computations done faster in the past. You get the latest, fastest processor in the market and that was all you needed to do. Lately, however, with the power requirements of yet faster single processors becoming highly uneconomical, the trend is instead towards building many smaller processors and then distributing heavy computations over these processors. The move from single-core and singleprocessor systems to multi-core and many-processors systems comes with the extra requirement of implementing computations in a way that can utilize these multiple computational units efficiently. This task of writing efficient parallel algorithms will not be possible without improving programming languages and compilers to provide the mechanisms to do so. In recent years substantial research effort is being spent on providing such mechanisms. This thesis work is one of these efforts. In this work we investigate how the available potential parallelism in Mathematical models can be used for efficient parallel computation.

Computer aided mathematical modeling and simulation is one of the most computationally intensive areas of computer science. Even simplified models of physical systems can impose a considerable computational load on the processors at hand. Being able to take advantage of the potential computation power provided by modern multi-core and many-processor systems is vital in this application area. 
Equation-based Object Oriented languages like Modelica [9] provide a very convenient way of modeling real world cyber-physical systems. Object orientation gives these languages the power to hierarchically model physical systems. This allows reuse and flexible modification of existing models and enables users to provide increasingly complex models by building on existing components or libraries. Complex models in turn require a lot of computational power to be conveniently usable. This is why parallelization in modeling and simulation is an area that needs extensive investigation. Simulation of ever more complex models will become too time consuming without efficient automatic and explicit methods of harnessing the power of multi-core processors.

In this thesis work we have studied the problem of extracting and utilizing parallelism from large task systems with heavy dependencies. This has been investigated, specifically, in the context of the Modelica equationbased object-oriented language and the OpenModelica [38] modelling and simulation environment. Some parallelization approaches have been studied in the past by the Programming Environments Laboratory (PELAB) here at Linköping University where the OpenModelica compiler is being actively developed. Most of these past parallelization approaches were concerned with automatic extraction of parallelism from Modelica models. There have been different prototype implementations that tried to provide automatic parallelization of simulations on multi-core CPUs as well as GPUs. Some of them were capable of simulating full physical models with no restrictions while others had certain restrictions on the system e.g. restrictions on the Modelica source code, restrictions on the solvers that can be used, etc. Unfortunately some of these implementations were rather obsolete by the time this thesis work started due to lack of maintenance or just simply because they were not relevant any more due to continuous changes to the OpenModelica compiler and recent improvements in the parallel programming arena. Other recent parallelization attempts are operational but differ in several ways from the work presented in the thesis. More information on these parallelization implementations and methods is given in Section 4.3 and Section 11.4.

We have also investigated an explicit parallelization approach by extending and improving the Modelica language and the OpenModelica compiler with support for explicit parallel programming constructs. There was virtually no effort on explicit parallel programming in Modelica prior to this work. The only such parallelization attempt is from 2006 [61]. That was extremely limited regarding the provided parallelization capabilities. Not to mention that the parallel programming world have advanced very much since that attempt. In this work we have tackled the problem of bringing modern parallel programming methods and approaches into modelling languages in general, and Modelica in particular, to take advantage of modern multi-core and multi-processor CPU and GPU architectures. 
This thesis work presents two independent but inter-operable approaches to parallelization of Modelica models evaluated on implementations in the OpenModelica compiler. Many of these results are valid for Equation-based Object Oriented languages and environments in general. The two approaches are:

- Automatic parallelization of equation-based models

- Explicit parallelization of algorithmic models

The first parallelization approach is a task-graph based method concerned with automatically extracting and utilizing parallelism from complex equation systems. This is a very compelling approach due to the fact that it can handle existing models and libraries without any modification. The method and implementation mainly consists of two different parts: a dependency analysis and parallelization extraction phase and a run-time task system handling and parallelization phase. The dependency analysis phase is rather specific to the compiler and simulation environment at hand, in this case OpenModelica. The runtime task-system handling and parallelization part, on the other hand, is implemented as an independent C++ library and can be used in any other simulation environment as long as the dependency information is readily available.

The explicit parallelization approach is more language and compiler specific. This approach introduces new explicit parallel programming constructs, for example parallel for-loops, to the Modelica language, implemented as extensions of the OpenModelica compiler. Using these extensions, users can write explicitly parallel algorithmic Modelica code to run on multi-core GPUs, CPUs, accelerators and so on. Even though this approach requires users to write their algorithmic Modelica code in specific ways, the effort is usually worthwhile since they can achieve much higher performance improvements for suitable algorithms. Moreover explicit parallel programming means that users are expected to have some knowledge of parallel programming. This might be an issue for modeling language users who are usually experts in fields other than computer science. However, with the increasing prevalence of multi-core processors, some knowledge of parallel programming is bound to be a necessity for anyone working with any programming language. The explicit parallel programming extensions are not yet standard Modelica and are currently only available for users of the OpenModelica compiler. To our knowledge there is no other Modelica tool that provides similar features at the moment of this writing.

\subsection{Research Problem}

Parallel or multi-threaded computation has become mainstream in the past decade. Specially the widespread availability of shared memory multi-core 
personal computers meant that quite a bit of the software, applications and algorithm that a normal end-user utilizes daily are multi-threaded. Many computer science application areas have adapted to this trend quickly and improved their implementations to take advantage the available computational power of personal computers to the full extent. Computationally intensive application areas such as image processing, video editing, video games and so on have been at the forefront of this development. Traditional scientific and mathematical computational software and frameworks have also started, albeit more slowly that the former ones, to widely adapt and provide methods for harnessing this processing power.

Mathematical Modelling and Simulation environments on the other hand have not been as quick to adapt to the changing trend. Of course Mathematical Modelling and Simulation is a relatively newer area compared to the veterans of computer science such as image processing. However, it is also one of the areas of computer science that can considerably benefit from adapting multi-threaded computations. Since multi-core computers have become such a common part of every computer users arsenal it is high time that Mathematical Modelling tools take advantage of their additional power.

This work has tried to investigate the feasibility, adaptability and usability of multiple parallel programming paradigms into the modern Mathematica Modelling and Simulation ecosystem. The work has tried to close the gap between the modelling tools and languages and modern multi-core and multiprocessor architectures. Adapting and incorporating multi-threaded capabilities into state-of-the-art Mathematical Modelling and simulation tools is a hot area of research in the corresponding community at the moment. This work is one of those efforts. At the start of this work none of the most popular Modelica Mathematical Modelling and Simulation tools provided neither automatic nor explicit parallelization capability. This means that there is an urgent and apparent need for such capabilities in order that users can take advantage of the computational power that they have at their disposal.

\subsection{Main Contributions}

The main contributions in this thesis are aimed at reducing the prevalent gap between Mathematical Modeling tools and approaches vs parallel programming approaches, frameworks and tools. Achieving this goal should allow for a more effective and efficient modelling and simulation of complex multidomain mathematical models.

This thesis investigates two independent yet inter-operable parallelization approaches, Automatic parallelization and Explicit parallelization. The contributions to each approach are briefly summarized below. 


\subsubsection{Automatic Parallelization}

The main contributions of the automatic parallelization effort can be summarized as:

- Evaluate the feasibility of Automatic Parallelization of the solution mathematical equation systems on modern shared-memory multi-core and multi-processor designs.

- Evaluate the feasibility of an automatic parallelization approach based on Transmission Line Modelling.

- Design of an automated dependency analysis of mathematical equation systems for automatic parallelization.

- Investigative adaptive, load balanced scheduling and execution of large and complex equation systems based on runtime profiling and cost analysis.

- Evaluation of basic clustering and scheduling routines for effective partitioning of equation systems for balanced multi-threaded computation.

- Evaluate the feasibility of adaptive runtime scheduling and loadbalancing of large highly dependent task systems of mathematical simulation computations.

In addition to answering the above theoretical contributions, the automatic parallelization part of this thesis work has contributed the following:

- Early design and implementation of an automatic parallelization approach based on Transmission Line Modelling.

- Design and implementation of the ParModAuto task system parallelization library for parallelization support in the OpenModelica compiler. The implementation provided:

- A high level, portable, flexible, extendible library for representation and manipulation of large task systems with heavy dependencies.

- Multiple clustering and scheduling heuristics for partitioning and executing task systems with variable cost tasks.

- Runtime profiling and rescheduling capabilities.

\subsubsection{Explicit Parallelization}

The main contributions of the explicit parallelization effort can be summarized as follows: 
- Investigation of the feasibility of adapting data parallel explicit parallelization paradigms of traditional languages to Equation-based Modelling and Simulation languages, specifically in the Modelica Language.

- Evaluation of the usability and flexibility of explicit parallel programming extensions for Mathematical Modeling Languages, specifically in Modelica,

- Evaluation of the performance of data parallel explicit parallelization over multiple application areas and implementations.

In addition to answering the above theoretical contributions, the explicit parallelization part this thesis work contributed:

- Design and implementation of the ParModelica Language extensions: A set of language extensions enabling integrated parallel programming in the Modelica language. This provides modern GPGPU programming inspired extensions such as:

- Parallel for-loops capable of evaluating loops using multiple threads.

- Parallel and kernel functions resembling OpenCL and CUDA counterparts.

- Routines and functions for complete control over thread management.

- Design, implementation, and evaluation of a runtime system supporting the ParModelica parallel extensions for parallel execution on multicore CPUs as well as GPUs.

\subsection{Practical Considerations}

Parallel programming is significantly more complicated and demanding than sequential programming. Incorporating parallelization, whether it is explicit or automatic, into a compiler makes things further complicated. The work done for this thesis is closely coupled to and mostly intended for the OpenModelica Modeling and Simulation Environment. As a result it is dependent on a number of features provided by the OpenModelica ecosystem. The OpenModelica compiler and all the other accompanying tools are constantly under active development. There are a number of developers actively working on different parts of the environment. This means that the implementations and changes we have to do to incorporate parallelization have to consider these developers. Initial implementations of parallelization in this work tended to change many of the existing features of the compiler. Specially in the back-end and the runtime system. However, this kind of changes, while 
relatively easier and quicker to implement, made the development process a bit more complicated for other developers. It has to be taken into account that these developers are not usually concerned with parallelization and probably are not familiar with parallel programming. As a result changes they make either breaks operations or they would have to wait and ask for what exactly new things are supposed to do and how to go about changing without affecting the expected parallelization operations.

It is easier to do an integrated implementation where everything is done as part of the compiler. Explicit parallelization is inherently language and complier specific. If extensions are to be added to the language then there is no way around modifying the compiler extensively. For extensions that are involved in parallelization, which requires runtime support as well, the whole compiler - from the lexer to the runtime system - needs to be modified or extended. There is no way around this. Automatic parallelization on the other hand, at least in general, requires a lot less direct modification to the core compiler in practice. Most of the heavy lifting can be done by a stand-alone implementation that provides the necessary functionality when it is needed. This approach, while increasing the amount of implementation effort needed, can considerably simplify the whole development process for developers who are not concerned with parallelization at all.

The implementations done are also not intended to be prototypes. These parallelization efforts are to be part of the complete OpenModelica ecosystem for regular usage. This means the implementation has to consider everything supported by the OpenModelica compiler and runtime. Of course not every functionality will be available or usable immediately. However, the work does have to consider eventually being able to compile and simulate any and every model - in addition to other features such as optimization and so on - that the normal OpenModelica work flow supports. This means simplifications that can be done by restricting what users can parallelize are not considered. One such example would be solver selection. The implementation should not make restrictions on what solvers the user can chose to simulate a given model. It has to support all solver options that the compiled model offers at runtime.

Most of the core OpenModelica compiler is implemented in a language called MetaModelica [89] [42] which an extended version of Modelica. However, the default runtime system of OpenModelica is written in the C language $^{1} . C$ is, of course, the preferred language of implementing runtime systems since it is a low level - these days $C$ is considered low level language - and runs everywhere. Despite this appeal, the runtime systems for both the explicit and automatic parallelization approaches of this work are written in $\mathrm{C}++$. Of course there might be systems or architectures that only support

\footnotetext{
${ }^{1}$ There is also a $\mathrm{C}++$ runtime system that has been in development. This runtime system is starting to become more complete and has started to provide near-complete support of the required functionalities.
} 
C. However, that was not enough of a motivation to implement features in C when $\mathrm{C}++$ has well tested and widely used libraries that can make the implementation process simpler, cleaner and more efficient. Any decent processor architecture that expects to utilize parallelization should have a $\mathrm{C}++$ compiler which is what we expect. However, we have not taken $\mathrm{C}++$ too far. The implementation uses very few $\mathrm{C}++11$ features. This extra $\mathrm{C}++11$ features have been available in most compilers for quite some time now and can be fairly expected to be supported. We have considered that as reasonable assumption since not all processor architectures get their compilers updated constantly.

There were some considerations and technical limitations that affected the Explicit parallel programming approach done in this thesis work. One of the biggest limitations for the explicit parallelization approach is that the compilers and tools used to compile the generated OpenCL [62] code are very restrictive. The OpenCL standard 1.2 used in this work is based on the C99 standard (ISO/IEC 9899:1999) [57] with many restrictions. For example only a few headers from the standard C library can be used in an OpenCL program. The OpenModelica compiler runtime requires many complex operations to be fully operational. This means that it makes quite heavy use of the $C$ header files as well as other utility libraries. In order to make sure that generated parallel OpenCL code is compilable while maintaining inter-operability with the normal sequential runtime environment has required many compromises. However, there have been some $\mathrm{C}++$ construct extensions of the core OpenCL language provided by some hardware vendors e.g. the OpenCL Static C++ Kernel Language Extension from AMD [2]. However, being vendor specific means that they are only available on certain architectures and are not really fully portable.

Furthermore, more recent versions of the OpenCL standard have considerably improved the language support by introducing the OpenCL C++ support into the core language [10]. These extensions can be used to improve the current implementation and can improve several issues. However, the default runtime system for the OpenModelica compiler still remains in C. This means in order to take advantage of the $\mathrm{C}++$ features the code generation for sequential and parallel portions would have to diverge considerably. This can lead to fragmented implementations where we will have to maintain two different code generation paths.

\subsection{Thesis Structure}

The thesis starts by providing a very brief background presentation regarding parallel programming in general in Chapter 2. Chapter 3 presents a short overview of Mathematical modelling and Simulation as well as related topics and methods. The rest of the thesis is divided into two main parts. Part I 
consists of chapters dedicated to Automatic parallelization and Part II consists chapters related to the Explicit parallelization approaches.

The first part presents the methods and approaches used to extract and implement automatic parallelization in equation-based task systems. A brief explanation of dependency analysis and extraction of parallelism from highly connected equation systems is presented. Then the features and implementation of the Task System Library used for parallelization of the resulting task systems are explained in detail. These include the clustering algorithms, schedulers, profiling and cost estimations methods and so on. This part of the thesis is partly based on the following two papers:

- Mahder Gebremedhin and Peter Fritzson

Parallelizing Simulations with Runtime Profiling and Scheduling

Proceedings of the 8th International Workshop on Equation-Based Object-Oriented Modeling Languages and Tools, (EOOLT'2017), Weßling, Germany, December 01, 2017.

- Mahder Gebremedhin and Peter Fritzson

Automatic Task Based Analysis and Parallelization in the Context of Equation Based Languages

Proceedings of the 6th International Workshop on Equation-Based Object-Oriented Modeling Languages and Tools, (EOOLT'2014), Berlin, Germany, October 9, 2014.

- Martin Sjólund, Mahder Gebremedhin and Peter Fritzson

Parallelizing Equation-Based Models for Simulation on Multi-Core Platforms by Utilizing Model Structure

17th International Workshop on Compilers for Parallel Computing (CPC 2013), Lyon, France, July 3-5, 2013, 2013.

The second part presents and explains the ParModelica algorithmic language extensions. The design of these constructs is inspired by OpenCL and is implemented as an extension of the Modelica language supported by the OpenModelica compiler. The extensions and the available mechanisms for runtime support of this explicit parallelization approach are explained in this part of the thesis and are based partly on these two papers:

- Gustaf Thorslund, Mahder Gebremedhin, Peter Fritzson and Adrian Pop

Parallel Simulation of PDE-based Modelica Models Using ParModelica

Proceedings of 9th EUROSIM Congress on Modelling and Simulation, Oulu, Finland. 2016.

- Mahder Gebremedhin, Afshin Hemmati Moghadam, Kristian Stavåker and Peter Fritzson

A Data-Parallel Algorithmic Modelica Extension for Efficient Execution on 
Multi-Core Platforms

Proceedings of the 9th International Modelica Conference (Modelica 2012), Munich, Germany. 2012.

- Afshin Hemmati Moghadam, Mahder Gebremedhin, Kristian Stavåker and Peter Fritzson

Simulation and benchmarking of Modelica models on multi-core architectures with explicit parallel algorithmic language extensions

Fourth Swedish Workshop on Multi-Core Computing MCC-2011, 2011.

Corresponding introductions, background information and previous work are presented in each part of the thesis.

Other publications by the author not used in this thesis work but are related to modeling and parallelization are:

- Alachew Shitahun, Vitalij Ruge, Mahder Gebremedhin, Bernhard Bachmann, Lars Eriksson, Joel Andersson, Moritz Diehl and Peter Fritzson Model-Based Dynamic Optimization with OpenModelica and CasADi IFAC-AAC 2013, 2013.

- Bachmann, Bernhard, Lennart Ochel, Vitalij Ruge, Mahder Gebremedhin, Peter Fritzson, Vaheed Nezhadali, Lars Eriksson, and Martin Sivertsson.

Parallel multiple-shooting and collocation optimization with OpenModelica. In Proceedings of the 9th International Modelica Conference (Modelica 2012), Munich, Germany. 2012 


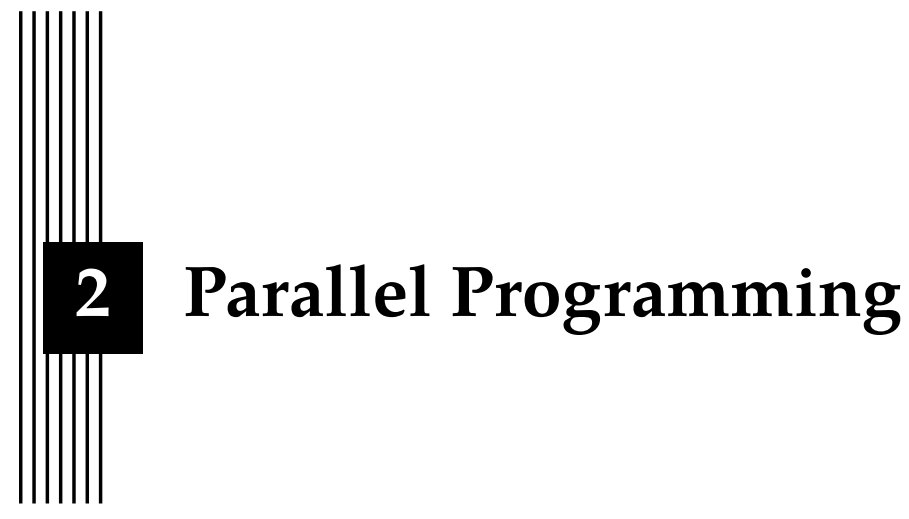

\subsection{Introduction}

Parallel programming is concerned with the simultaneous or parallel use of multiple computational units or resources to solve a given computational problem. A given computational problem can be broken down into smaller less computationally intensive problems and computed on different processing units with a system wide control of problem structures and coordination.

There are many different paradigms and flavors of parallel programming in existence today. Especially in recent years, with the advent of widespread availability of multi-core and multi-processor architectures, researchers are in a rush to provide and utilize even more efficient and powerful paradigms and implementations.

Of course there is no universally best solution to all the computational problems that exist in the physical world. Different kinds of applications require different approaches and implementations to take full advantage of the computing power of the available resources. Moreover, different processor architectures are suited for different paradigms and approaches.

User preferences and programmability are other important characteristics that are influencing the development of these parallel programming paradigms. Some approaches are intended for advanced users with a good knowledge of the problems of parallel programming who are looking to take the last drop of performance out of the computational resources available to them. Others approaches are intended for less experienced users looking for 
a quick and efficient way of improving the performance of their computations.

For the sake of classifying the work done in the scope of this thesis, we can categorize parallel programming methods/approaches in three ways. The first categorization is concerned with the programmability of the approach from a user's perspective. How will users be able to take advantage of the potential parallelization? Do they need to write their programs in a specific way? Will they have to modify existing code to take advantage of the method? The second categorization will be concerned with the memory model used in the parallelization approach. In this regard parallelization can be classified as shared memory or distributed memory approaches. The third and final categorization is based on the type of threading model or the types computations the approach is suitable for. Some parallelization paradigms are geared towards performing the same operations on a large amount of shared data sets - data parallelism while others are intended for performing possibly different tasks on possibly distributed data sets — task parallelism.

\subsection{Programmability}

With regard to programmability, we can classify parallelization approaches as automatic parallelization and explicit parallelization. These approaches are briefly explained in the next sections.

\subsubsection{Automatic Parallelization}

Automatic parallelization is the process of automatically detecting, optimizing, and parallelizing a computation. This parallelization method involves enhancements to compilers while the language stays the same. It imposes no or a quite small amount of work from the user's perspective. Users would not have to write their model or code in any different way than they would with no parallelization in mind. The compiler has full responsibility of finding and utilizing any potential parallelism from the user's model or algorithm.

Improving existing compilers for supporting automatic parallelization requires a considerable effort. However, it is naturally the most preferred way for end-users since it enables them to use models and algorithms without having to learn the details of complicated parallel programming languages. This is especially useful for communities like Modelica where most users working with the language/compiler are experts in a different field than Computer Science.

Another advantage of automatic parallelization approaches is that they allow the parallelization of existing code. The possibility of parallelized execution of existing libraries and implementation without any need for changes is quite appealing. Of course some sort of consideration might need to be made 
when writing code in order to assist the compiler with better extraction of parallelism.

\subsubsection{Explicit Parallelization}

Explicit parallelization, unlike automatic parallelization, is based on users explicitly stating where, when, and how their code should be parallelized. Explicit parallelization requires modifications to the compiler as well as to the language itself, i.e., if it doesn't have support for explicit parallelization yet, which currently is the case for the standard Modelica language.

To utilize this kind parallelization users have to write programs that uses these constructs explicitly where parallelism is needed. This means that users need to have some knowledge and expertise about how to write efficient parallel code.

Despite the fact that users have to spend extra effort in developing their programs to utilize explicit parallelism, this can result in huge performance improvements for many kinds of algorithms. Humans, at least for now, usually have a better understanding of their algorithms than the compilers. By implementing their programs or models in an optimized explicit way, they can achieve higher performance gains than the compiler would have done automatically.

\subsection{Memory Model}

One of the most common classifications of parallel programming has to do with the memory model in use by the computational machine. Generally speaking we can divide most computational systems capable of parallel processing into two different categories: shared memory systems and distributed memory systems.

\subsubsection{Shared Memory}

In traditional shared memory systems, all processors or any individual computational units can access the same main memory address space. This memory space is used for shared data storage. Most modern personal desktop and laptop computers from the biggest manufacturers such as Intel and AMD are based on shared memory multi-core CPU architectures. These multi-core machines have become a common household item. Due to this widespread availability parallel programming research focusing on shared memory multicore and multi-processor architectures is a very active field of research in the computer science community at the moment. This thesis work is one of those research efforts and focuses mainly on such architectures.

In addition to traditional shared memory CPU architectures, lately, it has become quite common to have so called General Purpose Graphic Processing 
Units (GPGPU). While graphical processing units were dedicated only to image and video processing operations in the past, recently it has become quite common to employ these architectures in the field of high performance scientific computations. In this architecture it is possible to have local and/or private memory spaces for data that is not needed to be shared. For example modern GPGPU based architectures have these hierarchical memory spaces to improve the overall performance of computations. Such GPGPU based architectures are one of the main focuses of explicit parallelization approach of this work together with more traditional CPU architectures. This is discussed in detail in Part II.

There are a number of parallel programming frameworks and platforms geared towards shared memory architectures. Frameworks and/or language extensions such as OpenMP [12] and POSIX threads (pthreads) [13] have been quite popular in parallel programming for shared memory CPUs. More recently with the widespread availability and increasing power of GPUs hierarchical memory heterogeneous frameworks such as the Open Computing Language (OpenCL) [64] and CUDA [82] have become popular. OpenCL is specially of interest in this work because it is the framework chosen to support the runtime implementation of the explicit parallelization approach presented in Part II. OpenCL is appealing since it is not vendor specific - as opposed to CUDA, its closest relative - and is capable of targeting a multitude of parallel processing devices such as CPUs, GPUs, Digital Signal Processors and so on.

All the work done as part of this thesis is aimed solely at shared memory architectures. While it is possible to replace the runtime systems, for both the automatic and explicit parallelization approaches, to one that uses a distributed memory framework this is currently not available and is not in near future plan.

\subsubsection{Distributed Memory}

In Distributed Memory multiprocessor systems, as the name suggests, individual processing units do not share access to the same memory space. Each processing unit has its own private memory and will perform computations using what is available in its own memory space. This means these individual independent processing units need to communicate required changes or updates to their memory spaces with other units. This requires that there should be a dedicated communication framework or protocol to make sure that required data available across these processing units is consistent at all times.

Message Passing protocols can be used to facilitate the communication process in distributed memory computations. These communication protocols make sure that data can be communicated in a consistent and reliable fashion. In addition, distributed memory systems need some sort of dedicated 
communication physical network to exchange data. The de-facto distributed memory parallel programming framework is the Message Passing Interface (MPI) library standard [95]. The Open MPI project [46] provides an opensource implementation of the MPI standard.

Distributed memory parallel programming has its advantages and disadvantages over the Shared memory variant. One of the biggest advantages being that it is relatively much more scalable. A Distributed Memory system can be extended by adding additional processing units and updating the network accordingly. This is why most very High Performance machines such as Super Computers and clusters are Distributed memory systems. Shared memory systems, on the other hand, are usually implemented in a single physical unit and are not as easily scalable.

\subsection{Threading Model}

Regarding threading models, we can classify parallelization approaches into data parallel and task parallel methods. These are briefly presented in the next sections. There is no clear-cut distinction between these two models of parallel computation. Computations are rather loosely attributed to each parallelization model based on how closely they resemble the corresponding pure model.

\subsubsection{Data Parallelism}

Data parallelism involves multiple computational units simultaneously performing the same or very similar operations to different items/parts of a given data set. Ideally this would involve every processing unit involved in the computation performing exactly the same operation on different data elements. A good example of data parallelism would be a simple element-wise addition of two vectors where each processing unit performs addition on the corresponding single elements from each of the two arrays. Of course not all processing units can perform exactly the same operation on different data for all physical problems. There are many cases where a few selected units might be doing additional operations or fewer operations based on the specifics of the problem at hand.

Data parallel programming usually involves operations on data structured into arrays and different processing units operating on these arrays. Since these operations are mostly similar, there are not as many parallel control structures (barriers, synchronizations) needed compared to task parallelism.

Most parallel architectures are designed with heavy data parallelism requirements in mind. This is especially true in recent years with the everincreasing power and complexity of multi-core CPU and GPGPU architec- 
tures. Data parallel computational model is the main focus of the Explicit parallelization work done as part of this thesis discussed in detail in Part II.

\subsubsection{Task Parallelism}

Task parallelism lies at the other end of the spectrum compared to data parallelism. In an ideal task parallel operation each involved computational unit performs a potentially completely different operation on the same or different data compared to other units. This is in contrast to data parallelism where all units perform the same or very similar operation on different parts of the data set.

Task parallel operations are commonly represented by a task graph which is usually a directed acyclic graph. This task graph represents the different operations to be performed as the nodes of the graph. Furthermore, the edges of the graph represent data and control dependencies between the individual tasks of the system. These dependences need to be obeyed to have a correct execution of the system. Large task systems with heavy dependencies are considerably more complicated and demanding than data parallel operations. There needs to be a specific set of mechanisms to ensure not just that the operation is performed correctly, but also to make sure performance does not suffer.

Task parallel programming model is used in this work to represent, extract and utilize parallelism in large complex equation systems resulting from physical systems modelled in equation-based mathematical modelling languages. This is discussed in detail in Part I of this thesis.

\subsection{Combined Shared Parallelism: Programmability with Threading Model}

The two classifications of parallelization models explained above can be combined and used to take advantage of different algorithms. For example, it is possible and common to have compilers extract data parallelism automatically or to have users write explicit task parallel programs.

In this work the explicit parallel programming extensions were designed mainly for data parallel operations. This is a direct consequence of the programming model they mimic, which is OpenCL. However, users can of course use some of these extensions to implement their task parallel algorithms.

On the other hand, the automatic parallelization methods and implementation presented here are, currently, limited to extracting task parallelism from complex equation systems. This does not mean that parallelization is always done as task parallelism. It simply means that right now the compiler doesn't look for and does not extract possible data parallel operations from 


\begin{tabular}{l|l|l} 
& Automatic & Explicit \\
\hline Data Parallel & & \\
\hline \hline Task Parallel & & \\
\hline
\end{tabular}

Table 2.1: Supported Parallelism

a given algorithm in a Modelica model. It is only concerned about finding dependencies at an equation level which can affect the dependency relationship for parallelization purposes. How the extracted parallelism is utilized is a different matter. Depending on the kind of scheduler and executor this task parallelism can be converted to a data parallel approach with tasks as data. The Level Scheduler implementation presented in Section 7.4.1 is a good example. Here clustered tasks within each level are represented as arrays of tasks and a simple data parallel iteration loop is used to execute this task array.

Table 2.1 shows what kinds of parallelism can be used with or are extracted by OpenModelica compiler for Modelica models at the moment of this writing only based on this work. To summarize:

- Users can write explicitly data parallel or task parallel algorithms.

- The compiler can currently extract task parallelism automatically from equation systems.

It is rather straight-forward to implement the missing parallelization which is automatically extracting data parallelism. For example it should be rather easy to locate arithmetic expressions like element-wise multiplication of two arrays which can benefit from data parallelism. Once these operations have been extracted the runtime functionality already available for the explicit data parallel implementation can be used to perform the rest of the work. 



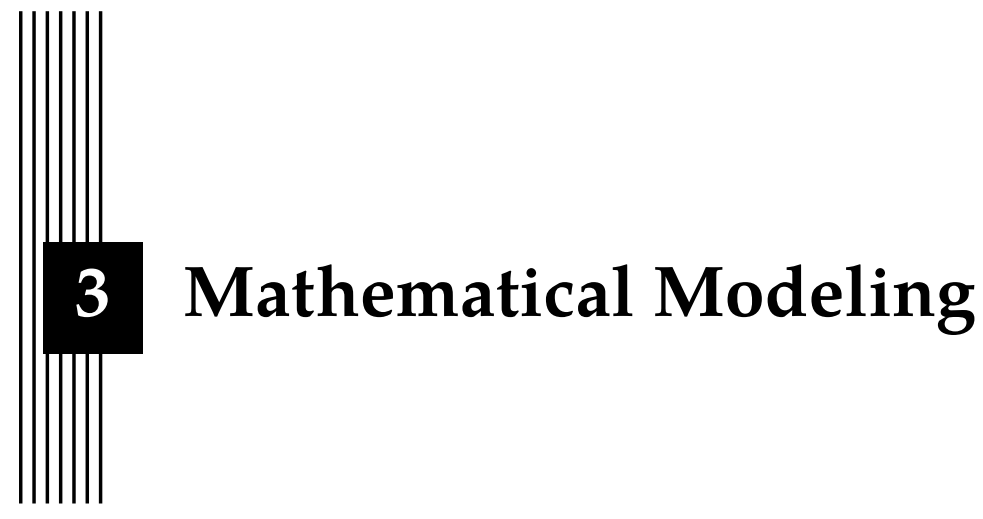

Computer aided Mathematical Modelling and Simulation is becoming more and more popular lately. The core concept of performing simulations of physical phenomena captured in the form of mathematical equations is by no means a new concept. However, in the past decade or so we have started to see wider adaptions of what are called Mathematical Modelling languages and corresponding tools. Yet the idea of modelling languages might still be an area that most programmers have never heard of or are not familiar with. Suppose you have to create computer models of some physical system, say an electrical circuit. The first thing that needs to be done would of course be to represent the problem mathematically using equations that represent the laws of physics governing the system. For an electric circuit these would be, for example, the rules of serial or parallel connections, the equations relating voltage, current and resistance in a resistor, Kirchhoff's laws and so on. For a translational mechanical system we have Newton's laws of motion. These equation systems will define the expected behaviour of the system and are what we consider a Mathematical Model.

If equation systems are to be solved, let's say by pen and paper, then we will use the equation manipulation techniques that the rules of Mathematics provides us to solve this equation system. We will first state what quantities/variables are known and which ones are to be computed or solved for. These manipulations can involve something as simple as the basic rules of elementary level algebra such as: subtracting the same quantity from both sides of an equality leaves us with a valid equality. It might also involve 
quite complex linear algebra operations such as using root finding methods to solve a system of coupled set of equations. What if we can use a computer to solve such equation systems? Surprisingly with most traditional programming languages or tools we will still have to do most of this preprocessing to be able to represent our problem algorithmically. This is needed mostly because of one simple fact. Traditional programming languages do not understand equations. Rather, they provide assignment statements. This is quite restrictive. For example assignments state what is known and what is unknown explicitly specified in the format. Any expressions in the right hand side are known quantities and the left hand side is the unknown, i.e assignments can be viewed as a restricted and special form of equations. If you change what is known in the system then you will have to re-write the algorithm. Your model is not reusable.

Of course there are modelling tools that alleviate the user from writing these basic equations systems for every physical system she or he wants to model. These tools usually provide pre-written or pre-modelled components that can be combined to form more complex systems there by reducing the amount of work needed. However, these are not programming languages. Users are always limited to the components already provided by the tool. This is usually the reason why most modelling and simulation tools are restricted to a specific field. They only provide components that are common in the specific field the tool is intended for. In contrast, with a programming language such as $\mathrm{C}++$ or Java, you could virtually model any physical mathematical phenomena albeit it would require a vast amount of additional effort.

Equation-based modelling languages try to reduce this gap between mathematical modelling and computer programming. Computers have become powerful, as have the algorithms that we run on them. If we can manipulate a given system of equations to represent it algorithmically to be solved with a given programming language, why shouldn't we let the computers do it for us? Our machines can manipulate equation systems much more quickly and more accurately than we can if we device the "meta-algorithms" for them to do so. This will mean that we can model perpetually bigger and more complex models with a relatively much less effort and error. Equation-based modelling languages and the accompanying compilers achieve exactly that and more.

How exactly does a modelling language capture equation systems? Of course an equation-based language will need to provide more constructs and concepts compared to other programming languages. It should be able to capture a physical phenomenon in a complete, reusable, maintainable and readable way to be advantageous over other languages. To achieve these properties a modern equation-based language should provide well known programming concepts such as Object-Orientation and add more mathematical concepts such as representation of equations, the concept of propagating time, rates of changes (derivatives), discrete behaviour and so on. The compilers 
and runtime systems for modelling languages also need to be able to process these equations and provide mathematical methods such as linear and nonlinear equation solvers, differential equation solvers and so on.

This chapter tries to cover the concept of mathematical modelling and related methods in a brief and concise way. This is meant to just give a quick overview of the concept and is by no means enough for a complete understanding. However, it tries to present the basic concepts that the reader needs to become familiar with the mathematical modelling approach and with the tools and methods used/involved in this document. We assume that the reader has basic understanding of any normal programming languages such as $\mathrm{C} / \mathrm{C}++$, Java, Python and similar as well as a basic familiarity with differential equations and linear algebra.

\subsection{Assignments and Equations}

Before going into discussions about mathematical modelling and modelling languages in detail it might be important to address one aspect of mathematics and programming notations, specifically the meaning of the equalitys sign $=$ and the relationship between mathematical systems of equations and computer programs based on assignments. Consider this simple formulation

$$
a=a+5
$$

Does this equation/statement specify that $a$ is equal to 2.5 or does it say that we need to increment $a$ by 5 ?

\subsubsection{Notations}

$$
\begin{array}{r}
x=1, \\
y=2, \\
y=x+3
\end{array}
$$

Equation (3.2) should appear inconsistent, even at first glance, for most people past elementary grades. $y$ is clearly not equal to $x+3$ which happens to be 4 . Now consider the listing below which should look fairly familiar for most people who have some experience with computer programming.

$$
\begin{aligned}
& \mathrm{x}=1 ; \\
& \mathrm{y}=2 ; \\
& \mathrm{y}=\mathrm{x}+3
\end{aligned}
$$

What is important is that most people will not find anything wrong with the above "code". This code is almost exactly the same as Equation (3.2) except for the formatting and the semicolons ${ }^{1}$. The set of equations/assignments

\footnotetext{
${ }^{1}$ Note that even with commas or without commas at all, the code will still be valid in some languages. However that is besides the point since they are extra here.
} 
here is interpreted differently depending on whether we consider it a Mathematical Equation System/Computer Program.

If you have ever written a computer program in one the most popular languages since the 1960s, the chances are you have used = sign as some sort of operator. In most languages $=$ is used as an assignment operator. Others have "correctly" used other symbols to represent assignment, e.g, ALGOL uses :=, LISP uses set ...

Most modern languages, specially those that are descendants of FORTRAN, seem to have settled on $=$ as an assignment operator thanks to the popularity of $\mathrm{C} / \mathrm{C}++/$ Java family of languages.

Languages are conventions. Including the constructs making up our programming languages. All words are made-up words. As are signs. There is nothing intrinsically special about = that makes it represent equality, say more than $\|, \$$ or $a$. It is just a convention made at some point in the past. So as long as there is an agreement on what it represents, any symbol can be used to represent equality, equally. However, it would be very convenient if the symbol chosen has the same meaning in mathematics as it does in programming languages considering the tight relationship between the two.

\subsubsection{Equality Checks}

It should be noted that checking for equality and stating equality are two different concepts in mathematics and programming. In $\mathrm{C} / \mathrm{C}++$, for example, two concatenated equality symbols " $=="$ are used to check for equality. This is not stating that the two expressions involved in the statement are equal. It is merely checking if the two expressions are equal as a result of any statements we have made or equations we have stated earlier.

In these languages " $=="$ is a query posing a question that could either be true or false while " $="$ is a statement saying that we should update the left hand side to be equal to the right hand at that point. On the hand in a mathematical equation " $=$ " states that the right hand side and the left hand side are equal at all times.

\subsubsection{Equations}

$$
\begin{aligned}
& a=2 \\
& b=4 \\
& c=a+3 \cdot b \\
& d=4 \cdot a+2 \cdot c
\end{aligned}
$$

Imagine if some physical phenomena presented itself mathematically as Equation (3.3). It is rather straightforward to translate this system, we can call it sys1, into a computer function/algorithm that computes the values of the 
unknowns in the system. The variables $a$ and $b$ can be initialized to their respective values and $c$ and $d$ are computed using their respective assignments.

\section{Listing 3.1: Algorithm for Equation 3.3}

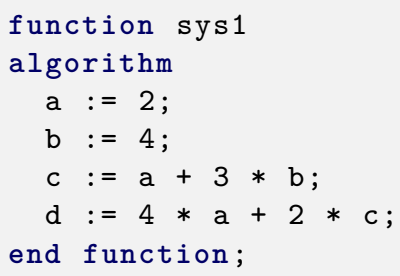

In this case the computer function/program was derived directly from the original system without any preprocessing. Now consider the alternative, but equivalent, representations of Equation (3.3) shown in Equation (3.4) and Equation (3.5)

$$
\begin{aligned}
& c=a+3 \cdot b \\
& a=2 \\
& b=4 \\
& d=4 \cdot a+2 \cdot c
\end{aligned}
$$

$$
\begin{aligned}
& a=2 \\
& b=4 \\
& a=c-3 \cdot b \\
& d=4 \cdot a+2 \cdot c
\end{aligned}
$$

Although logically wrong, both of these representations are semantically valid algorithms. The only problem with these two representations is that they do not provide a consistent solution to the equation system. That is, the values obtained after evaluating the sequence of assignments do not satisfy every equation in the original system if they are to be evaluated in the specified sequence. For example, assuming uninitialized variables are set to $0^{2}$, Equation (3.4) results in $[0,2,4,8]$ for $[c, a, b, d]$ respectively. This solution does not satisfy the first equation in Equation (3.4), i.e., $0 \neq 2+3 \cdot 4$.

We can formulate an interpretation for these two invalid algorithms in terms of immutable variables. An immutable variable cannot be modified once it is created, i.e., it can only be initialized. This form of formulation is also usually refereed to as Static Single Assignment form. We can now say that every assignment creates a new variable. A variable can take a name that has

\footnotetext{
${ }^{2}$ This might be considered undefined behaviour in some languages. For the discussion here we can assume that the UB resulted in value 0 for these cases.
} 
previously been taken by another variable, making the previous variable no longer accessible, or even lie in the same memory address overwriting it. It is nonetheless a new variable. With this formulation, Equation (3.4) and Equation (3.5) can for example be rewritten as Equation (3.6) and Equation (3.7) respectively. ${ }^{3}$

$$
\begin{aligned}
\underline{i} & =0 \\
\underline{j} & =0 \\
c & =\underline{i}+3 \cdot \underline{j} \\
a & =2 \\
b & =4 \\
d & =4 \cdot a+2 \cdot c
\end{aligned}
$$

$$
\begin{aligned}
a & =2 \\
b & =4 \\
c & =0 \\
\underline{i} & =c-3 \cdot b \\
d & =4 \cdot \underline{i}+2 \cdot c
\end{aligned}
$$

Therefore, there is nothing intrinsically invalid with interpreting/translating Equation (3.4) and Equation (3.5) as algorithms. When fully interpreted as algorithms, they just represent different equation systems than they do when treated as equations. Algorithms written in most traditional programming languages have certain properties that are interesting when compared to equation forms, such as:

- They are written in one specific form. The assignment form defines each variable.

- They are usually optimized for memory/space. Variables that are not referenced past the current assignment can have their names (hence the memory space) reused.

- Variables referenced before their defining equations are initialized with random values, i.e., new assignments are introduced defining them with random values.

Thus the question becomes how to transform a given system of equations to a valid algorithmic representation.

Translating these two systems to an algorithm that results in a valid solution for the equation system they actually represent requires some preprocessing or transformation. For example Equation (3.4) needs to reorder the equations to bring Equation (3.4a) to the third place so that it would not use uninitialized variables $a$ and $b$. As for the second representation, we need to solve for $c$ by transforming Equation (3.5c).

\footnotetext{
${ }^{3}$ Again assuming uninitialized variables get the value 0 by default. We can also use a function $U B()$ to defined these assignments. It would not matter here since the equations will still be structurally consistent.
} 
Such transformations of mathematical equations for solving with the help of traditional (read as assignment-based) programming languages is quite common. At least for small systems. It is also usually performed by a human.

We can formulate Listing 3.1 as a set of ordered functions whose outputs are the set of all variables assigned to by each assignment.

$$
\mathbf{y}=\left[\begin{array}{l}
a \\
b \\
c \\
d
\end{array}\right]=\mathcal{S}()=\left[\begin{array}{l}
f_{1} \\
f_{2} \\
f_{3} \\
f_{4}
\end{array}\right]=\left[\begin{array}{c}
2 \\
4 \\
a+3 \cdot b \\
4 \cdot a+2 \cdot c
\end{array}\right]
$$

In this specific example $\mathcal{S}$ has no inputs (See Section 3.1.4) since the function is completely self contained. Accounting for inputs we can generalize Equation (3.8) as the explicit function $\mathcal{S}: \mathbb{R}^{n} \rightarrow \mathbb{R}^{m}$ given by

$$
\mathbf{y}=\mathcal{S}(\mathbf{u})
$$

where $\mathbf{y}$ is the vector of outputs, i.e., all the variables assigned to by all the statements in the function. $\mathbf{u}$ is a vector of inputs, i.e., any variable whose value is provided outside the function. Equivalently, with immutable variables, an input is a variable that used but not assigned to by the statements of the function.

All the different representations (Equation (3.3), Equation (3.4) and Equation (3.5)) of the original equation system can be normalized to a canonical representation of equations

$$
\begin{aligned}
a-2 & =0 \\
b-4 & =0 \\
c-a-3 \cdot b & =0 \\
d-4 \cdot a-2 \cdot c & =0
\end{aligned}
$$

We can now formulate Equation (3.10) as a set of ordered functions over $\mathbf{y}$ as

$$
\begin{gathered}
0=\mathcal{Q}(\mathbf{y})=\left[\begin{array}{l}
f_{1} \\
f_{2} \\
f_{3} \\
f_{4}
\end{array}\right]=\left[\begin{array}{c}
a-2 \\
b-4 \\
c-a-3 \cdot b \\
d-4 \cdot a-2 \cdot c
\end{array}\right] \\
\mathcal{F}(X)=\left[\begin{array}{c}
f_{1}(X) \\
f_{2}(X) \\
\vdots \\
f_{n}(X)
\end{array}\right]= \begin{cases}f_{1}\left(x_{1}, x_{2}, \ldots, x_{n}\right) & =0 \\
f_{2}\left(x_{1}, x_{2}, \ldots, x_{n}\right) & =0 \\
\vdots & \\
f_{n}\left(x_{1}, x_{2}, \ldots, x_{n}\right) & =0\end{cases}
\end{gathered}
$$


In the general form, accounting for inputs, we can generalize such systems of equations with the implicit function given by

$$
0=\mathcal{Q}(\mathbf{y}, \mathbf{u})
$$

An equation-based language is a language that can capture a system of equations in implicit form given by Equation (3.13) or any other variations of representations such as those shown in Equation (3.4) or Equation (3.5). By contrast, a traditional assignment based language is only able to capture an explicit equation system of Equation (3.9). Listing 3.2 shows one of the many possible equation-based representations of Equation (3.10). This equationbased formulation needs to be transformed to the correct assignment-based form of Listing 3.1 to be solved.

Listing 3.2: Equation-based program for Equation

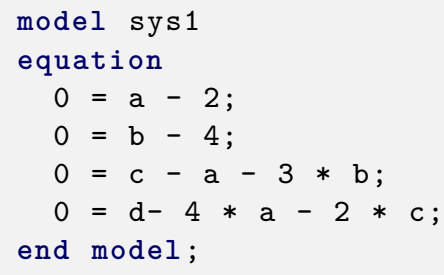

\subsubsection{Inputs and Outputs}

A normal algorithmic function, say in $\mathrm{C} / \mathrm{C}++$, can optionally have inputs and outputs. One way to interoperate these inputs and outputs into our formulation of equations based languages is as follows. Inputs to a traditional function can be seen quite simply as initializations of the input variables as local variables by the quantity the input holds at the start of evaluation. Inputs are nothing but additional equations with an equal number of additional variables in our equation system. The system remains consistent. This means, if we are inclined, we can actually write Equation (3.13) as

$$
0=\mathcal{Q}(\mathbf{y})
$$

where y now includes all the input variables in addition to local ones and $\mathcal{Q}$ includes all the corresponding equations.

As for outputs, every variable of an equation system is an output. It just happens that we filter out some of those variables and make them available for other parts of the computation. In other words traditional assignment based function is just a wrapper around the statements (algorithm) or the 
body. It takes all the outputs of the assignment (all assigned variables) and filters out some of the variables making only a subset of them available to the outside.

\subsubsection{Solving Equation Systems}

So far we have discussed an equation-based formulation that is able to capture mathematical models in a flexible form. However, the question of solving such equation-based formulations remains unaddressed. We cannot use our computers directly to solve formulations of Equation (3.13) such as Listing 3.2 for example.

The most general approach to solving such systems of equations would be to employ iterative root finding methods. Perhaps one of the most popular and fairly efficient of such methods is Newton's Method (See Section 15.1). While such root finding methods are generally applicable for solving complex systems of equations, they can be quite inefficient specially as the number of equations grows larger. However, if we are able to symbolically analyse the system of equations we can usually find certain mathematical properties that can make solving it a lot more efficient and consistent.

For example one of the most important characteristics such a system can have is Linearity. If the system is linear, i.e, if all the individual equations are linear, such as the one shown in Equation (3.10), it can be written in matrix form as follows:

$$
0=\mathbf{A} \cdot \mathbf{y}+\mathbf{b}
$$

Equation (3.10) can for example be written as shown in Equation (3.16). Such systems of linear equations can in general be solved with standard numerical algorithms such as Gaussian Elimination. Such specialized algorithms for specific systems are generally more efficient and consistent than root finding algorithms.

$$
\left[\begin{array}{l}
0 \\
0 \\
0 \\
0
\end{array}\right]=\left[\begin{array}{cccc}
1 & 0 & 0 & 0 \\
0 & 1 & 0 & 0 \\
-1 & -3 & 1 & 0 \\
-4 & -2 & 0 & 1
\end{array}\right] \cdot\left[\begin{array}{l}
a \\
b \\
c \\
d
\end{array}\right]+\left[\begin{array}{c}
-2 \\
-4 \\
0 \\
0
\end{array}\right]
$$

The system of equations shown in Equation (3.16) actually shows another vital property of equation systems that can make the solving process quite straight forward. In addition to being a linear system, the coefficient matrix (a.k.a. incidence matrix), $\mathbf{A}$, is Lower Triangular, i.e., all entries above the diagonal elements are 0 . In other words if we solve for the diagonal elements in each corresponding row without changing the order of rows, no variable 
will be used before it is defined or computed. All the diagonal elements can computed using a procedure called Forward Substitution as follows

$$
\begin{aligned}
y_{1} & =-b_{1} / a_{11} \\
y_{2} & =-\left(b_{2}+a_{21} \cdot y_{1}\right) / a_{22} \\
y_{3} & =-\left(b_{3}+a_{31} \cdot y_{1}+a_{32} \cdot y_{2}\right) / a_{33} \\
\vdots & \\
y_{n} & =-\left(b_{n}+a_{n 1} \cdot y_{1}+a_{n 2} \cdot y_{2} \cdots+a_{n, n-1} \cdot y_{n-1}\right) / a_{n n}
\end{aligned}
$$

This procedure can be generalized as

$$
y_{i}=\left(b_{i}-\sum_{j=1}^{i-1} a_{i j} \cdot y_{j}\right) / a_{i i}
$$

Lower Triangular forms are very important structures in symbolic manipulation techniques for solving equation systems. Advanced symbolic manipulation methods essentially try to structure the system into a Lower Triangular form. Of course, it is not always possible to do so. Either the whole system or parts of it can be a coupled Linear or Non-Linear System of equations where it is not possible to solve for a variable using previously computed values alone. These blocks need to be solved using linear or non-linear methods as a whole. In that case they try to transform the matrix into Block Lower Triangular form. In practice equation-based modelling tools such as OpenModelica usually employ graph theory based approaches instead of matrix transformations to find matchings and sort equation systems. These methods are discussed briefly in Section 4.2.

The details of transforming or finding a mapping of a generalized equation system to an equivalent algorithmic procedure is, not surprisingly, fairly complicated. However, for now all we need to know is that it is a mechanical operation that can be performed by a computer function. For the sake of discussion we will just use a generalized ideal root finding function, $\mathcal{R}$, that can take a system of equations in the form of Equation (3.13) and solves for $\mathbf{y}$.

\subsection{Dynamic Systems: Time}

The equation-based language we have so far can actually model a real-world physical phenomena described by its equations. However, we can only model so called static systems. The formulation of Equation (3.13) is static because it will always result in the same values of the variables for the same set of inputs no matter when we evaluate it. More specifically we can say that it has no mechanism to represent progress.

Many of the real-life phenomena we usually want to model mathematically involve change over time. If we take one arbitrary evaluation of the system, we would like to see differences in the values of the variables between 
this initial evaluation and the next one. Of course, we can change the inputs given to the system on each distinct evaluation. That means we can emulate time in our system by changing one real valued input, $t$, by a finite amount, $\delta t$ every time we evaluate or solve the system since that initial evaluation. With time in consideration we now generalize Equation (3.13) for dynamic systems as

$$
\mathcal{Q}(\mathbf{y}, \mathbf{u}, t)=0
$$

where $t$ is the special independent value representing relative time. A mathematical time-dependent simulation is a successive evaluation/solution of a mathematical equation system at successive time points.

For our equation-based language model, $\mathcal{Q}$ representing some mathematical model, a root finding function $\mathcal{R}$, we can now formulate a simple simulation as shown in Listing 3.3.

\section{Listing 3.3: A simple simulation}

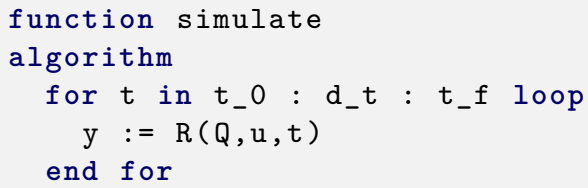

\subsection{Rate Of Change: Derivatives}

A lot of real life problems usually involve the rates of changes of some physical quantity. This can happen into forms. First we can have the quantities of some variable over time and we would like to compute the rate of change of this quantity over time as well. Secondly nature (or our measurements) provides us with the rate of change of some quantity and we would like to know the cumulative effect of this quantity over some interval, that is we want to compute the values of the variable from its rate of change. For example Newton's Second Law of motion is stated as

$$
\begin{aligned}
F & =m \cdot a \\
a & =\dot{v} \\
v & =\dot{s}
\end{aligned}
$$

where $m$ is the mass of a given object, $F, a, v$, and $s$ are the force applied to, the acceleration, the current velocity, and the distance travelled by the object respectively. Given the velocity, we might want to compute the acceleration 
(the rate of change) or the displacement (the cumulative effect) of this variable. Such a ubiquitous law of simple translational motion involves rates of change of quantities that are of interest. Therefore, it is essential that our mathematical modelling formulation is able to comfortably represent and handle such physical systems involving rates of changes or derivatives of variables. How do we represent a derivative? We can simply add a new operator. For example, we can add an operator with a function syntax $\operatorname{der}(v)$ signifying the derivative of variable $v$. A mathematical model of the system can now be described as shown in Listing 3.4.

\section{Listing 3.4: Second Law}

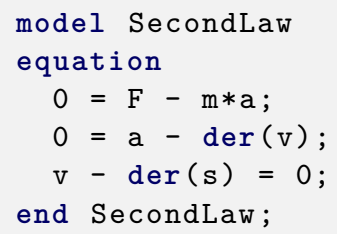

Accommodating for derivatives of variables we can extend Equation (3.19) to

$$
\mathcal{Q}(\dot{\mathbf{x}}, \mathbf{y}, \mathbf{u}, t)=0
$$

where $\dot{x}$ is a vector of all the derivatives in the system. The quantity of a variable and its derivative are tightly related. The derivative of a function $x=f(t)$ at a point $a$ can be approximated by 4

$$
\left.\dot{x}\right|_{a}=f^{\prime}(a)=\lim _{h \rightarrow 0} \frac{f(a+h)-f(a)}{h}=\lim _{h \rightarrow 0} \frac{x_{a+h}-x_{a}}{h}
$$

We can use this relationship to compute $\dot{x}$ from $x$ numerically using successive evaluations of Equation (3.19), i.e., numerical differentiation. The smaller $h$, the more accurate our approximations become.

However, often we are interested in computing $x$ from $\dot{x}$ instead. In many physical systems the rate of change of some quantity is readily available and we are interested in computing the cumulative value of the quantity from this rate of change. Such a formulation can be derived from the Fundamental

\footnotetext{
${ }^{4}$ For simplicity assuming a continuous function $f(x)$.
} 
Theorems of Calculus. More specifically we have

$$
\begin{aligned}
\int_{a}^{b} \dot{x} d t & =x_{b}-x_{a} \\
x_{b} & =x_{a}+\int_{a}^{b} \dot{x} d t
\end{aligned}
$$

If we have an initial estimate $x_{a}$ for the value of $x$ at $t_{a}$ then we can approximate the value $x_{b}$ at $t_{b}$ using Equation (3.23) with the help of either symbolic or numerical integration methods to compute the second operand of the right hand side. There are a number of well studied and implemented numerical integration methods. Some of these methods are discussed in Section 15.2.

The relationship between the derivative variables and the differentiated variables allows us to compute one from the other. Therefore, we consider either $\dot{x}$ (the derivatives) or $x$ (the differentiated variables) as knowns depending on whether we are employing differentiation or integration respectively. Considering the relationships between the derivatives and the differentiated variables, i.e, those whose derivatives appear in the system, we can write Equation (3.21) as

$$
\mathcal{Q}(\dot{\mathbf{x}}, \mathbf{x}, \mathbf{y}, \mathbf{u}, t)=0
$$

where

- $\mathrm{x}$ : State variables or states: A vector of all ${ }^{5}$ the variables whose derivatives appear in the system.

- $\dot{x}$ : State derivatives: A vector of the derivatives of the state variables in the system.

- $\mathrm{y}$ : Algebraic variables: A vector of all the variables of the system that are not states.

- $\mathbf{u}$ : Input variables: A vector of all the variables not defined/computed by the system. The values for these variables are provided from outside. They can be dynamic inputs, parameters or constants.

- $t$ : time: Is the independent variable.

For our equation-based language model, $\mathcal{Q}$ of Equation (3.24) representing some mathematical model, a root finding function $\mathcal{R}$, and a numerical integration method $\mathcal{I}$ of the form Equation (3.23), our simple simulation scheme now involves solving Equation (3.25) at every time step.

$$
\begin{aligned}
0 & =\mathcal{Q}(\dot{\mathbf{x}}, \mathbf{x}, \mathbf{y}, \mathbf{u}, t) \\
\mathbf{x} & =\mathcal{I}(\dot{\mathbf{x}}, \mathbf{x})
\end{aligned}
$$

\footnotetext{
${ }^{5}$ In practice, some variables might not be picked as states even though their derivatives appear in the system. This is beyond the discussion here.
} 
That is, we use the system of equation to solve for the algebraic variables, $\mathbf{y}$, and state derivatives, $\dot{\mathbf{x}}$, using the known values $\mathbf{x}, \mathbf{u}$ and $t$. Once the state derivatives, $\dot{\mathbf{x}}$, are computed we can then plug them into our integration method to get the next values of $\mathbf{x}$.

Employing a root finding function $\mathcal{R}$ to solve Equation (3.25a), our simple simulation program of Listing 3.3 can now be extended accordingly as shown in Listing 3.5

\section{Listing 3.5: A simple simulation}

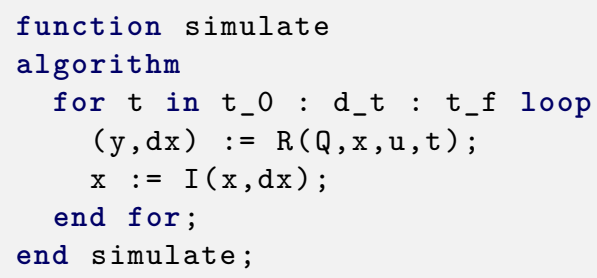

One important property that can be noticed from our simple formulation of a continuous simulation shown in Listing 3.5 is that successive evaluations of the simulation loop are not independent any more. This was the case when we did not incorporate differential equations into our formulation.

\subsection{Discrete Behaviour}

So far we have assumed that all variables are continuous. The systems have a well defined and expected behaviour governed by the equations that define them and the laws of physics. The quantities involved and the system does not change its behaviour suddenly. However, real life systems are not always isolated. There are usually disturbances that can cause the system to behave in a way that does not follow the expected behaviour. This sort of systems that are prone to sudden change are known as discrete Systems.

This does not mean that these changes are undesired. They are usually expected and accounted for in the formulation of mathematical models and are known in the modelling and simulation literature as Events. Some of these events might instantaneously change the quantity of one or more aspects of a system. For example a sudden increase in the voltage supply of an electrical circuit is one such discrete change. Other events might even affect the underlying structure of our mathematical model by instantaneously changing the system of equations governing the model. An electrical switch being turned 
on or an air conditioning unit changing from heating mode to cooling mode are examples of such sudden change.

In addition, some events are tied to a specific time in the simulation process. That is the exact time of such sudden changes is known a priori. For example a timed circuit switch turning on at a specific time is such an event. These are know as Time Events. On the other hand, there are events that are dependent on the quantities involved. An air conditioning system switching its operation mode due to the current ambient temperature in a room is one example. Such events are known as State Events.

The formulation of Equation (3.24) does not account for discrete behaviour of systems. We will not, however, go into more detailed discussion of events and discrete behaviour here. It should just be noted that a complete mathematical modelling language and associated tools should provide a well formed and consistent constructs and/or features to tackle and study such discrete behaviours.

\subsection{Modelica}

Modelica $^{6}$ is a non-proprietary, object-oriented ${ }^{7}$, equation-based, multi domain modelling language for component-oriented modelling of complex physical systems containing, e.g., mechanical, electrical, electronic, hydraulic, thermal, control, electric power or process oriented subcomponents. Its development is managed by the non-profit Modelica Association. The Modelica Association also overlooks the development of the open source Modelica Standard Library which contains model components and example models from different application domains.

\subsubsection{Modelica for Mathematical Modeling}

Modelica is quite well suited for modelling of complex physical systems. This is not surprising since the language was designed and continuously improved for that specific purpose. Like many other complex object oriented languages Modelica has classes, support for advanced features like inheritances (extends), operator overloading, generic programming (redeclaration) and so on. However, what makes Modelica especially well suited for modelling is its ability to capture physical systems in an intuitive way.

Probably the most important feature that makes Modelica so powerful is its support for equation-based acausal modelling. In addition to assignment statements which are common in most programming languages, Modelica allows equation-based formulations similar to what is discussed in the previous sections and so much more including support for discrete and mixed

\footnotetext{
${ }^{6}$ https://www.modelica.org/

${ }^{7}$ Object-oriented as in encapsulation/modularity, abstraction, inheritances, generics and so on. Who is to say what Object-oriented is: http://wiki.c2.com/?HeInventedTheTerm
} 


\section{Figure 3.1: A simple pendulum}

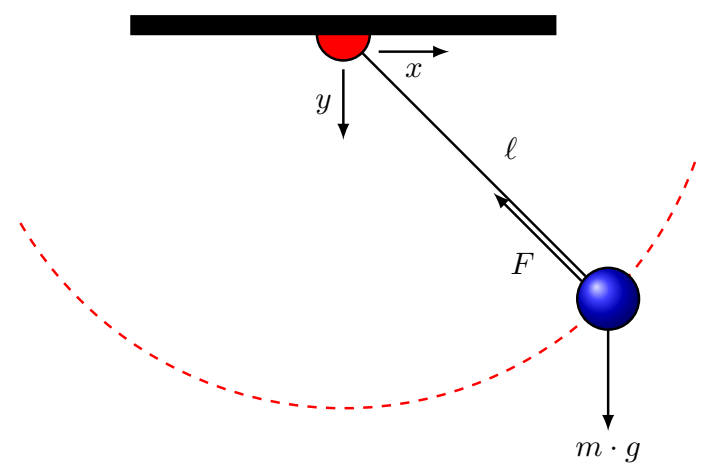

systems. It is the specific Modelica compiler's job to symbolically process the equation systems and transform the system into a causalized representation based on assignments (See Section 4.2 for brief discussion). This makes for a very flexible modelling environment where models can express physical systems in a natural way.

The simple ideal pendulum example of Figure 3.1 demonstrates how Modelica can be quite convenient in modeling physical systems. The equations governing the movement of the pendulum are shown in Equation (3.26). Comparing this the corresponding equation-based Modelica model shown in Listing 3.6. As we can see the Modelica model can be written in a way that resembles exactly the system of equations defining the physical system. The Modelica compilers take these representation and transform it to a formulation suitable for evaluating. In addition to symbolically transforming the equation system, a Modelica compiler/tool will also take care of numerical methods such as solving linear or non-linear systems of equations as well applying the corresponding integration mechanisms for finding a solution to the differential equations of the system.

$$
\begin{aligned}
m \cdot \dot{v_{x}} & =-\frac{x}{\ell} \cdot F \\
m \cdot \dot{v_{y}} & =m \cdot g-\frac{y}{\ell} \cdot F \\
\dot{x} & =v_{x} \\
\dot{y} & =v_{y} \\
x^{2}+y^{2} & =\ell^{2}
\end{aligned}
$$




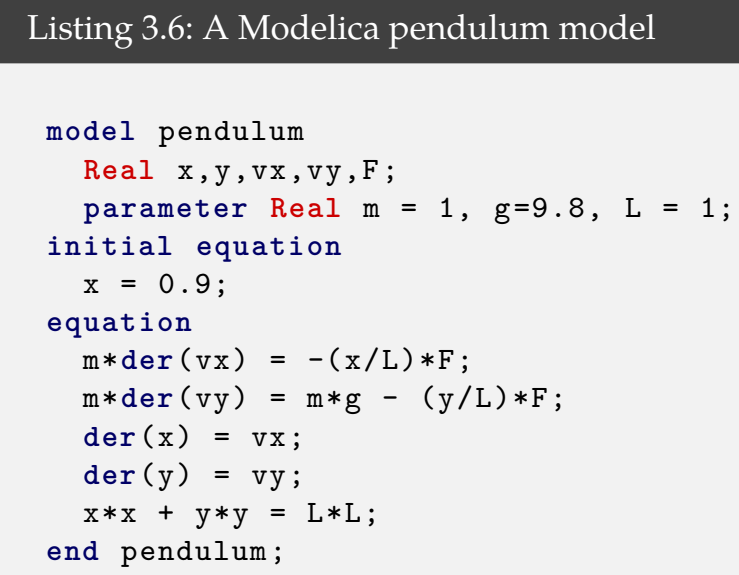

Modelica is an object-oriented language. This means that it can be used to create models in a hierarchical manner by combining and extending different model components. In addition, having different specialized classes such as models, records, blocks, connectors, etc., gives Modelica the ability to represent physical components in a way that resembles their real world attributes. For example record classes are used to represent a collection of data about a physical component for example A record class for a point in space contains the $\mathrm{x}, \mathrm{y}$, and $\mathrm{z}$ co-ordinates of the point. Model classes are used to represent components with dynamic behaviours. A model class for a rocket can contain the variables that represent its dynamic behaviour, e.g., its current mass, velocity, acceleration, etc. together with the equations that govern the behaviour of the rocket. Connector classes and connect equations enable modellers to create relationships and interactions between components in an intuitive way. Connector classes, connectors and connect equations enable modellers to create relationships and interactions between these components in an intuitive way.

There are many other Modelica features that are interesting for physical modelling [27] [29] [39]. Very detailed explanations and examples can be found in [36] [35] [100] and the Modelica Language Specification [9].

There are a number of modelling and simulation tools that allow physical modelling with Modelica. Some of these are commercial tools such as Dymola [17], Wolfram System Modeller [40] [75], Simulation X [97], MapleSim [74], IDA Simulation Environment [1] and others. There are also a few OpenSource tools such as JModelica.org [5] and OpenModelica [37] [43] [41]. 


\subsection{Modelica Standard Library (MSL)}

The Modelica Standard Library [8] provides model components that are based on standardized interface definitions. The library provides models in a variety of domains including Electrical (Analog, Digital and Machines), Mechanical, Thermal, Fluid, Magnetic and Media models as well as some common numerical functions. It is available freely and is usually bundled with most Modelica tool distributions. The library is quite extensively used and well tested. The development is overseen by the Modelica Association.

There are many more Modelica libraries from different vendors and users. Some of these are developed by the Modelica Association, some are free and others are commercial libraries. This extensive collection of libraries provides a very convenient and flexible modelling and simulation ecosystem where users can incorporate, extend and modify existing components to build bigger and more complex models. An extensive list of available Modelica libraries can be found at the Modelica Association website ${ }^{8}$.

Selected models from the Modelica Standard Library version 3.2 are used to test the performance of the implementations presented in this thesis.

\subsection{OpenModelica}

OpenModelica is an open-source Modelica-based modeling and simulation environment intended for industrial and academic usage. Its long-term development is supported by a non-profit organization The Open Source Modelica Consortium (OSMC) ${ }^{9}$. The Programming Environments Laboratory (PELAB) at Linköping University, together with OSMC, is developing the OpenModelica Compiler (OMC) for the Modelica language (including the MetaModelica [89], ParModelica [48] and Optimica [4] language extensions) and the accompanying simulation environment.

The research implementations developed in this thesis work are all done in and around the OpenModelica Compiler. The implementations and additions to the compiler presented here have been developed within several phases of the compiler. This is a direct consequence of the specific parallelization paradigm and programmability intended in each investigation. A rough depiction of the OpenModelica compiler's compilation phases is shown in Figure 3.2.

The Automatic parallelization approach presented in Part I required some modifications to the Back-end and Code Generator stages of the compiler. However, most of the work for this approach was put into the runtime support of parallel execution by providing the rather independent Task Systems Library presented in Chapter 8.

\footnotetext{
${ }^{8}$ https://www.modelica.org/libraries

${ }^{9}$ https://openmodelica.org/home/consortium
} 


\section{Figure 3.2: OpenModelica compiler's compilation phases}

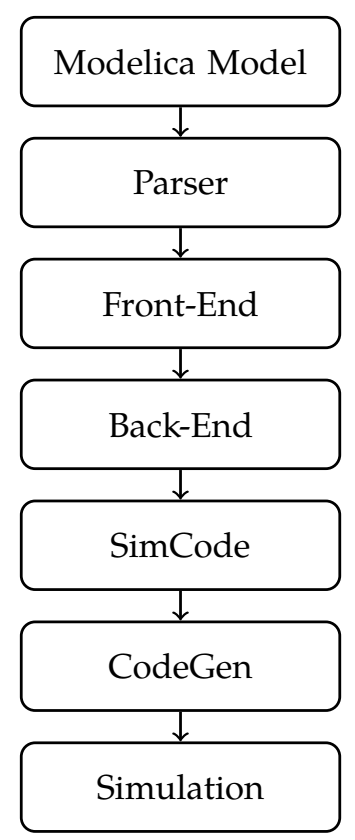

On the other hand, the explicit parallel programming extensions presented in Part II have required modification to almost all phases of the compiler starting from the parser all the way to the code generation and runtime support. Obviously adding new constructs to a language means that the compiler will have to recognize the new constructs, make sure that any syntactic and semantic rules for these constructs are obeyed, generate appropriate code for the usage, and finally provide runtime support for any new features required. The compiler has been modified for these changes.

Although the implementations of this work are in and around the OpenModelica compiler and accompanying tools, it should be noted that the ideas and methods developed can be applied to other tools, languages and modelling approaches. The Automatic parallelization approach and the accompanying task system parallelization implementation has taken the extra measures of making sure that it is not too tied with the OpenModelica even in the face of extra work and complexity. It is designed with minimal and reasonable assumptions about what is expected from tools that would want to utilize or incorporate it.

The Explicit parallelization approach is highly integrated into the OpenModelica compiler. Naturally extending a language with new constructs requires directly modifying the compilers. However, the technically speaking 
improvement is made to the language and not the compiler. The new constructs and approahces introduced for enabling parallelization in the Modelica language as a whole are not specific to the OpenModelica compiler. 


\section{Part I}

Automatic Parallelization 



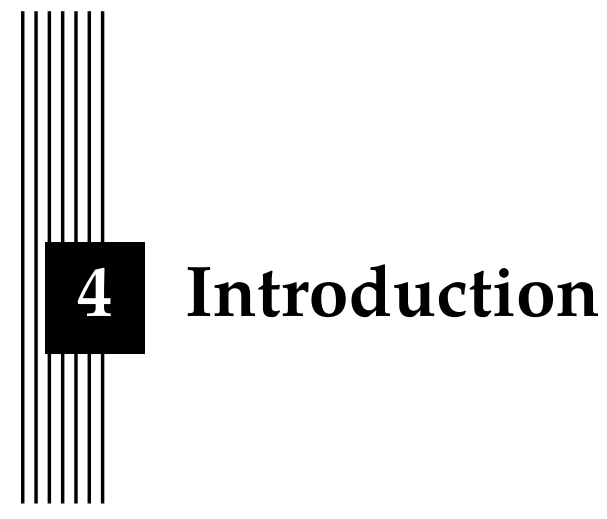

Automatic parallelization, in general, is a very appealing approach because it requires no additional effort from the user's/programmer's perspective. In addition, it requires little or no modification to existing code in most cases. Of course to take full advantage of the automatic parallelization capabilities of a compiler or tool it might sometimes be necessary to write computer programs in a specific way. However, compared to explicit parallelization this is almost always a much less time consuming and error prone procedure. Many programmers also are not well familiar with parallel programming methods and tools. Therefore, it's vital that more compilers and programming environments provide automatic parallelization capabilities.

Automatic parallelization support in mathematical modelling and simulation environments and tools is an even more appealing area. Specifically because most users of such tools are usually not computer science experts. Rather, most of them are experts in their modelling areas with little or no knowledge of advanced programming techniques let alone parallel programming methods. We can say this from first hand experience with such modellers. The explicit parallelization extensions of this work presented in Part II, for example, are not used as much as we had hoped. Modellers and engineers do not want to spend time formulating and debugging parallel implementations of their algorithms when they have the actual modelling and physical properties to worry about. This is probably the reason why most modelling and simulation tools are graphical drag-and-drop style or block based modelling approaches. 
This part of the thesis (Part I) presents automatic parallelization related concepts and methods investigated and implemented in this work. It is organized as follows. The current chapter presents a very brief overview of graph theory related approaches into the so called causalization of equation systems. The subsequent chapters present the two automatic parallelization approaches investigated. Chapter 5 presents a parallelization approach based on connected component analysis. Chapters 6 to 9 present a parallelization approach based on strongly connected component analysis.

The parallelization approaches taken in this work depend on multiple graph structures and related algorithms. Before going into a discussion of the causalization process and parallelization of equation systems, we will start by briefly describing basic graph structures and concepts for the sake of terminology consistency.

\subsection{Terminology}

For the sake of consistency we define some terminology around graph related aspects for the rest of the discussion.

\subsubsection{Graphs}

A Graph, $G$, is the pair $(V, E)$, where $V$ is a finite, non-empty set of vertices and $E$ is a set of pairs of vertices $(u, v)$ called an Edge. An Undirected Graph is a graph where the edges are symmetric, i.e.

$$
(u, v) \equiv(v, u)
$$

For any edge $(u, v) \in E, u$ and $v$ are called neighbours and they are adjacent to each other. For an edge $e=(u, v)$, vertices $u$ and $v$ are incident to the edge $e$.

A path $\tilde{\mathcal{P}}$ in $G$ is a sequence of vertices $\left\{v_{1}, v_{2}, \ldots, v_{k}\right\}$ such that $\left(v_{i}, v_{i+1}\right) \epsilon$ $E, \forall v \in \mathcal{P} / v_{k}$. A simple path $\mathcal{P}$ is a path where no vertex appears more than once.

A cycle $\tilde{\mathcal{C}}$ is a closed path, i.e., a path $\left\{v_{1}, v_{2}, \ldots, v_{k}\right\}$ where $\left(v_{k}, v_{1}\right) \in E$. A simple cycle $\mathcal{C}$ is a simple path $\left\{v_{1}, v_{2}, \ldots, v_{k}\right\}$ where $\left\{v_{k}, v_{1}\right\} \in E$.

For the rest of this document the terms Path and Cycle refer to simple paths and simple cycles respectively.

A graph, $\mathrm{G}$, is said to be disconnected if the set of vertices, $V$, can be split into at least two disjoint sets $V_{1}, V_{2} \ldots V_{n}$ with no adjacent vertices between each set. Otherwise, the graph is said to be connected.

The disjoint sets $V_{1}, V_{2} \ldots V_{n}$ of a disconnected graph are the connected components of the graph. If an undirected graph is connected then there is a path from every vertex to every other vertex. 
A connected undirected graph with no cycles, an acyclic undirected graph, is a Tree. If the acyclic graph is disconnected then it is a Forest.

\subsubsection{Directed Graphs}

A Directed Graph (digraph) is a graph where the $E$ is a set of ordered pairs of vertices $(u, v)$, i.e.,

$$
(u, v) \not \equiv(v, u)
$$

A Simple Directed Graph is a graph which has no self loops and no parallel edges, i.e.,

$$
\forall(u, v) \in E, \quad u \neq v \wedge|(u, v)|=1
$$

For the rest of this document the term Directed Graph refers to Simple Directed Graphs unless it is explicitly stated otherwise.

For any edge $(u, v) \in E, u$ is called the parent of $v$ and $v$ is a child of $u$. The set of child vertices of a vertex $u$ is denoted by $\mathcal{F}(u)$ and the set of parents of $u$ is denoted by $\mathcal{B}(u)$, that is,

$$
\begin{aligned}
& v \in \mathcal{F}(u) \Longleftrightarrow(u, v) \in E \\
& v \in \mathcal{B}(u) \Longleftrightarrow(v, u) \in E
\end{aligned}
$$

A vertex $v$ is said to be reachable from another vertex $u$ if there is a path from $u$ to $v$.

A Directed Acyclic Graph (DAG) is a directed graph with no cycles. A vertex $v$ of a directed graph, $\mathrm{G}$, is minimal if and only if $v$ is not reachable from any other vertex. A vertex $v$ is minimum if and only if every other vertex is reachable from $v$. A minimum vertex is also refereed to as the root vertex.

The distance between two vertices, $u$ and $v$, of directed graph is the number of edges on the path from $u$ to $v$. The level of a vertex $v$ is the maximum distance from the minimum or root vertex. In other words the level is the cardinality of the longest path out of all the paths from the minimum vertex to $v$.

A topological sort of a directed acyclic graph is a list of all the vertices such that each vertex $v$ appears earlier in the list than every other vertex reachable from $v$.

A path and cycle in a directed graph are defined similar to undirected graphs except for the fact that edges now have directions. Connected and disconnected directed graphs as well as connected components of a directed graph are also defined similar to undirected graph.

A Strongly Connected Component (SCC), $S$, of a directed graph is the set of vertices where every vertex $v \in S$ is reachable from every other vertex $u \in V$. A vertex that does not belong to any bigger SCC is considered an SCC by itself. All cycles are part of a strongly connected components. A strongly connected component has at least one cycle. 


\subsubsection{Bipartite Graphs}

A Bipartite Graph, $G=(V, E)$, is an undirected graph where the set of vertices $V$ can be partitioned into two disjoint sets $A$ and $B$ such that no edge in $\mathrm{E}$ has two vertices in the same partition.

$$
\forall(u, v) \in E, \quad u \in A \wedge v \in B
$$

A balanced bipartite graph is a bipartite graph where the two partitions $A$ and $B$ have the same cardinality, i.e., $|A|=|B|$.

A Matching, $M$, in a graph $G$ is the set of edges where no two edges share the same vertex. If an edge $(u, v)$ is in a matching $M$ then both $u$ and $v$ cannot be in any other edge in M. A bipartite matching $M$, subsequently, is the set of edges that connect the partitions $A$ and $B$ with no shared vertex.

A Maximal Matching is a matching where no more edges can be added without introducing a shared vertex. A Maximum Matching (Maximum Cardinality Matching) is a matching with the largest cardinality.

A Perfect Matching is a matching where every vertex of the graph is part of the matching. A perfect matching, therefore, is a Maximum Matching and is only possible in graphs with even number of vertices. That means perfect matching in a bipartite graph is possible only if the graph is balanced.

A bipartite graph with partitions $A$ and $B$ has a perfect matching if and only if it satisfies Hall's Theorem (also knows as Hall's Marriage Theorem). Let $S$ be a subset of vertices in $A$ and let $\Gamma(S)$ denote the set of all vertices in $B$ that are adjacent to any vertex in $S$, Hall's theorem states that

$$
|\Gamma(S)| \geq|S| \quad \forall S \subset A
$$

is a necessary and sufficient condition for the existence of a perfect matching in a bipartite graph. That is no subset, $\mathrm{S}$, of the either partitions has fewer neighbours than the cardinality of the set itself. Balanced bipartite graphs that do not satisfy Hall's theorem are usually referred to as Structurally Singular Systems in modelling and simulation literature.

A bipartite graph with no cycles has a unique perfect matching if it satisfies Hall's condition, otherwise it has no perfect matching. This follows from the fact that an acyclic bipartite graph is a tree. However, not all bipartite graphs that have cycles and also satisfy Hall's condition have multiple perfect matchings, e.g. half bipartite graphs have a unique perfect matching. In fact any graph that has a unique perfect matching is a subgraph of a half bipartite graph [49].

\subsection{Causalization of Equation Systems}

Causalization of equation systems is the process of transforming systems of equations given in implicit form to their fully explicit or semi-explicit forms 
for a more efficient and numerically stable solution formulation. As we have seen in Section 3.3 equation-based mathematical modelling languages such as Modelica allow the flexible implicit representation of differential algebraic equation systems governing our physical problems. We need to transform such equation-based formulations to their assignment based forms using th so called symbolic manipulation methods.

Recall our formulation of systems of differential algebraic equations as given in Section 3.2, i.e.,

$$
\begin{aligned}
0 & =\mathcal{Q}(\dot{\mathbf{x}}, \mathbf{x}, \mathbf{y}, \mathbf{u}, t) \\
\mathbf{x} & =\mathcal{I}(\dot{\mathbf{x}}, \mathbf{x})
\end{aligned}
$$

where $\mathbf{x}$ is a vector of state variables - variables whose derivatives appear in the system and are considered known since they are computed by the integrator,$- \dot{\mathbf{x}}$ is a vector of state derivatives, $\mathbf{u}$ is a vector of input variables - variables which are defined outside the system and are considered known - , and $t$ is an independent variable incremented by a finite amount every time the system is evaluated, in this case representing time. Generally speaking, we can solve such a system of possibly non-linear equations by using root finding methods such as Newton's method for multi-variable systems discussed in Section 15.1. However, this is not always the best approach. There are specific structures of these systems of equations that can make the solution process much stable and efficient. The aim of causalization is, essentially, to try and transform Equation (4.7a) to an explicit formulation such as

$$
(\mathbf{y}, \dot{\mathbf{x}})=\mathcal{F}(\mathbf{x}, \mathbf{u}, t)
$$

so that we can breakdown the system into assignment based algorithmic formulation to solve it instead of solving it as a whole.

Consider the simple RLC circuit shown in Figure 4.1. The circuit is made up of for elements namely a sinusoidal voltage source, $V_{0}$, an inductor with inductance $L$, a capacitor with capacitance $C$ and a resistor with resistance of $R$. We are interested in finding the voltage across and the current through each of these four elements. The circuit can be described by the system of eight equations shown in Equation (4.8) using the voltage-current rules for each element and Kirchhoff's circuit laws. ${ }^{1}$

\footnotetext{
${ }^{1}$ Of course, there are multiple ways of formulating the equations for the system. We have specifically tried to follow the automated manner in which drag-and-drop style modelling tools generate equations for such systems to demonstrate some concepts.
} 


\section{Figure 4.1: A simple electrical circuit}

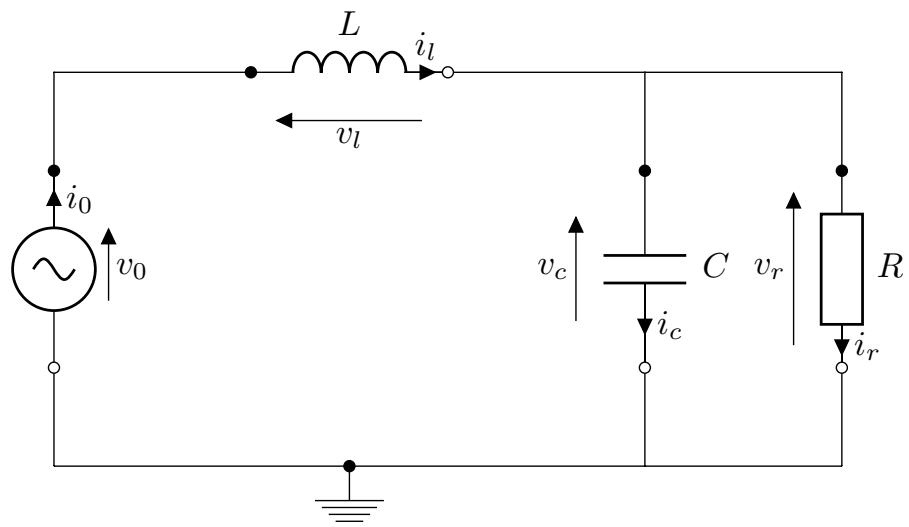

$$
\begin{aligned}
v_{0}=f(t) & \equiv & v_{0}-f(t)=0 \\
v_{l}=L \cdot \frac{\mathrm{d} i_{l}}{\mathrm{~d} t} & \equiv & v_{l}-L \cdot \frac{\mathrm{d} i_{l}}{\mathrm{~d} t}=0 \\
i_{c}=C \cdot \frac{\mathrm{d} v_{c}}{\mathrm{~d} t} & \equiv & i_{c}-C \cdot \frac{\mathrm{d} v_{c}}{\mathrm{~d} t}=0 \\
v_{r}=R \cdot i_{r} & \equiv & v_{r}-R \cdot i_{r}=0 \\
v_{r}=v_{c} & \equiv & v_{r}-v_{c}=0 \\
i_{0}=i_{l} & \equiv & i_{0}-i_{l}=0 \\
v_{0}=v_{l}+v_{c} & \equiv & v_{0}-v_{l}-v_{c}=0 \\
i_{l}=i_{c}+i_{r} & \equiv & i_{l}-i_{c}-i_{r}=0
\end{aligned}
$$

\subsubsection{Matching}

Causalization of such systems starts with a matching stage where we decide which equation should be used to solve for which variable. To do so, we first have to figure out which variables are considered known and which are not. Since we are using numerical integration methods to compute $x$ from $\dot{x}$, we consider all the variables whose derivatives appear as known. In this case $v_{c}$ and $i_{l}$ would be considered known and instead we would like to find equations to solve for $\frac{\mathrm{d} v_{c}}{\mathrm{~d} t}$ and $\frac{\mathrm{d} i_{l}}{\mathrm{~d} t}$. This means we have system of eight equations with the eight variables $\left\{v_{0}, v_{l}, \frac{\mathrm{d} v_{c}}{\mathrm{~d} t}, v_{r}, i_{0}, \frac{\mathrm{d} i_{l}}{\mathrm{~d} t}, i_{c}, i_{r}\right\}$. 
The system can now be represented by a bipartite graph where one set of vertices represents the equation while the other set represents the variables, and the edges signify the fact that a variable is used in the corresponding equation. This is shown in Figure 4.2a. The problem of finding equations to solve for a specific variable is now a bipartite matching problem. The bipartite graph satisfies Hall's condition which means it has a perfect matching, i.e., the system is not structurally singular. In addition, the graph happens to be acyclic which that means we have one, and only one, possible perfect matching.

The bipartite matching problem is a well studied problem. One of the most popular algorithms is the Hopcroft-Karp algorithm [54] which solves the problem in $\mathcal{O}(\sqrt{|V|},|E|)$ time in the worst case. An improved $\mathcal{O}\left(|V|^{1.5}, \sqrt{\frac{|E|}{\log |v|}}\right)$ implementation of the Hopcroft-Karp algorithm is also presented in [6]. There also algorithms proposed for finding all the perfect matchings of a bipartite graph [44] [45].

Just for the sake of completeness we would like to mention that the bipartite matching problem can be formulated as a special instance of the more general Minimum Cost Maximum Flow problem. This is a formulation that tries to find the maximum flow that can be sent from one node to another node in a general network graph where each edge has an associated capacity - the maximum flow that can traverse that edge - , and a cost - the cost of sending a unit flow through that edge. We can add a source node connected to all vertices on left hand side and a sink node connected to all the right hand vertices of the bipartite graph. The bipartite matching problem can now be formulated as a maximum flow problem where each node has a capacity and cost of 1 unit. There are number of algorithms for solving the maximum flow problem such as Edmonds-Karp's $\mathcal{O}\left(|V|,|E|^{2}\right)$ algorithm [26] and Dinic's $\mathcal{O}\left(|V|^{2},|E|\right)$ algorithm [23]. While it might appear unnecessary complicated at first glance, such a formulation can be quite helpful in solving some common issues in matching of mathematical equation system such as solvability issues (at least in cyclic graphs where we have multiple matchings) and state selection. We believe that the mathematical modelling and simulation researchers should look into possible adaptations of such formulation instead of pure bipartite formulations.

Getting back to our circuit system, the matching of the system is shown in Figure $4.2 \mathrm{~b}$ where the red lines signify the matched equations and variables, i.e., which equation will be used to solve for which variable.

\subsubsection{Sorting}

The next step in the causalization process is sorting. We can orient the graph into a directed graph where the equations form the vertices of the graphs and the variables make up the edges. If an equation uses a variable it is not 


\section{Figure 4.2: Bipartite graphs RLC circuit equations}

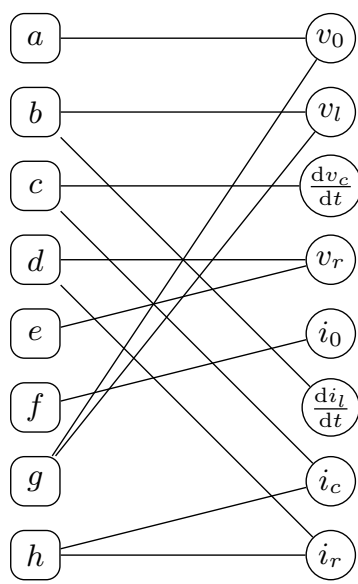

a. Original

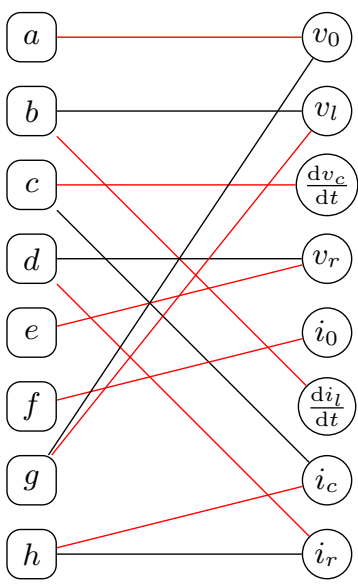

b. Matched

\section{Figure 4.3: Directed Graph of matched RLC circuit equations}
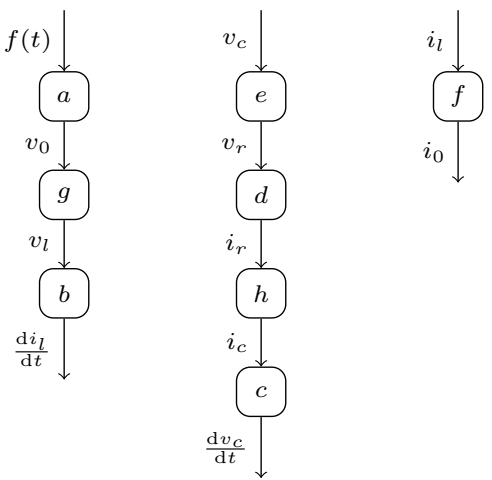

matched with, i.e. it has an edge to the variable that is not part of the matching, then it will have an incoming edge from the equation that defines that specific variable. The resulting directed graph following this procedure is shown in Figure 4.3.

We can now perform a topological sorting of the directed graph to obtain a sequence of executable assignment statements that can be used to solve the system within each time step. One possible topological sorting of the directed graph of Figure 4.3 is shown below in Equation (4.9). 


$$
\begin{aligned}
v_{0} & =f(t) \\
v_{l} & =v_{0}-v_{c} \\
\frac{\mathrm{d} i_{l}}{\mathrm{~d} t} & =\frac{v_{l}}{L} \\
v_{r} & =v_{c} \\
i_{r} & =\frac{v_{r}}{R} \\
i_{c} & =i_{l}-i_{r} \\
\frac{\mathrm{d} v_{c}}{\mathrm{~d} t} & =\frac{i_{c}}{C} \\
i_{0} & =i_{l}
\end{aligned}
$$

In this static single assignment form every variable now has a specific assignment used to compute it and no variable is used before it is defined assuming initial values are given for the state variables $v_{c}$ and $i_{l}$. The values of the derivatives $\frac{\mathrm{d} v_{c}}{\mathrm{~d} t}$ and $\frac{\mathrm{d} i_{l}}{\mathrm{~d} t}$ can be plugged into our numerical integration method to obtain the next values of $v_{c}$ and $i_{l}$ respectively.

\subsection{Automatic Parallelization Approaches}

Throughout our discussions of general mathematical modelling, simulation and symbolic processing of equation systems, we have come across a number of places where a tool can employ automatic parallelization. We can roughly classify automatic parallelization possibilities in mathematical equation-based modelling depending on where and how they utilize parallelism. A summary of the classifications is shown in Figure 4.4 (The shaded areas are the parallelization approaches investigated in this work). Each of these approaches will be discussed briefly.

\subsubsection{Parallelization Over Method}

One possible opportunity to utilize parallelization is at the integration/differentiation methods. The integration/differentiation methods used in assisting the solution process of differential algebraic systems can be implemented in a way that takes advantage of multiple processing units.

Consider the explicit $3^{\text {rd }}$ order Adam-Bashforth method discussed in Section 15.2.2.

$$
x_{n+1}=x_{n}+\frac{h}{12}\left(23 f\left(t_{n}, x_{n}\right)-16 f\left(t_{n-1}, x_{n-1}\right)+5 f\left(t_{n-2}, x_{n-2}\right)\right)
$$




\section{Figure 4.4: Parallelization Opportunities}

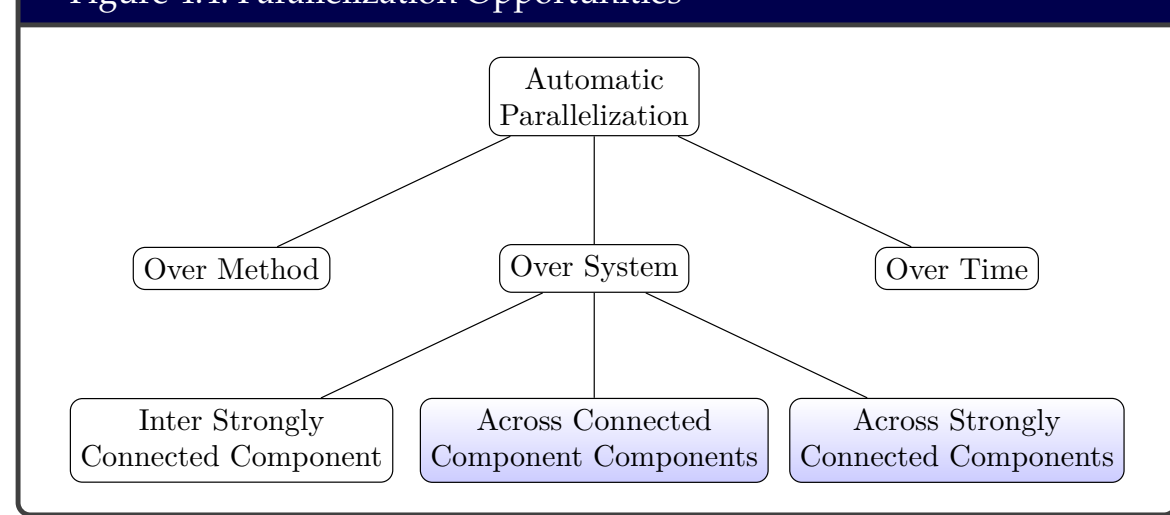

At first glance we can see that there are multiple evaluations of the function $f$, which represents our causalized equation system of equations such as Equation (4.9), on every step of this formulation. This evaluation can be done in parallel. Fortunately this happens to be unnecessary in general because the results of each previous evaluation, $f\left(t_{n-1}, x_{n-1}\right)$ and $f\left(t_{n-2}, x_{n-2}\right)$, could simply be memorized by the implementation removing the need for re-computation. Same applies for the current evaluation, $f\left(t_{n}, x_{n}\right)$, which can be memorized and used for the next step, as $f\left(t_{n-1}, x_{n-1}\right)$ that is. There are, however, other integration methods that compute intermediate values of $f(t, x)$ within each time step for a better approximation of the solution. These intermediate values are different in each time step and cannot be reused. One such method is the popular Runge-Kutta integration method. Higher order Runge-Kutta methods can be formulated in such a way that multiple stages of the computation are independent from each other leading to an opportunity to compute these stages in parallel [101] [91].

Another approach to parallelizing of integration methods involves the parallelization of linear algebra methods employed by the method. Consider the $3^{\text {rd }}$ order Adam-Moulton method discussed in Section 15.2.3

$$
x_{n+1}=x_{n}+\frac{h}{12}\left(5 f\left(t_{n+1}, x_{n+1}\right)+8 f\left(t_{n}, x_{n}\right)-f\left(t_{n-1}, x_{n-1}\right)\right)
$$

As noted in the discussion, this is an implicit method since $x_{n+1}$ is used in the right hand side of the formulation, i.e., it is not possible to solve for $x_{n+1}$ directly. Non-linear root finding methods such as Newton's method need to be employed in the solution of this formulation on every time step. It is possible to employ parallelized implementations of Newton's method in this case to improve the computation efficiency. For example the evaluation of the Jacobian matrix (See Section 15.1 and Equation (15.5)) as well as the linear solvers used in solving of Equation (15.7) can benefit greatly from parallelization. 
There are a number of parallelized solver implementation for ordinary as well as differential algebraic equation systems available. Such approaches are not the focus of this work and will not be discussed in any more detail.

\subsubsection{Parallelization Over Time}

Another parallelization approach in simulation of equation systems is the so called parallelization over time. This approach, essentially, involves computation of multiple $x$ values in parallel. This means evaluating an equation system, such as the one in Equation (4.9), for multiple time step simultaneously. Clearly this approach is not applicable for classical numerical integration methods including all those discussed in Section 15.2. All of theses methods depend explicitly on previous values of either $x$ or $\dot{x}$ to compute a solution for a give time step. Hence, they are intrinsically sequential in their formulation and are not suitable for parallelization over time.

A relatively new family of numerical integration methods known as Quantized State System (QSS) methods [105] [65] [21] [34] replace the time discretization of the classical integration methods with the quantization of the state variables. Further discussions and implementations of such methods can be found in [31] [11]. A parallelization approach of QSS methods for ordinary differential equations is presented in [32]. [33] provides an extensive study of parallelization approaches for QSS based simulation, which give additional possibilities for parallelization depending on the kind of model that is simulated.

\subsubsection{Parallelization Over Equation System}

Parallelization of the equation system is the focus of this work. This approach involves evaluating the computation of the equation system itself in parallel instead of parallelizing the integration methods. For example the assignments in the simple RLC circuit system of Equation (4.9) can be computed in parallel by utilizing the structure of the system. Parallelization approaches of the equation system can be further classified into three parts. These are discussed briefly below.

Parallelization within a Strongly Connected Component A strongly connected component of a directed graph is the set of components that are reachable from each other. In equation systems of mathematical models these SCCs (of at least two elements) represent a system of equations that are coupled together and cannot be transformed into a causalized assignment form.

Solving of these strongly connected components requires the use linear algebra methods. If the equations making up the strongly connected component are all linear equations then a linear solver method such as Gaussian Elimination needs to be applied to obtain a solution. If the system is made up 
of non-linear equations, instead, then there will be a need to use root finding methods such as Newton's method. Either way these bot of these methods can benefit from parallelization similar to the parallelization over method approach.

There are a number of tools, libraries or implementations that provide parallelized versions of these linear algebra methods, [53][22][94] and many more. Incorporating parallel evaluation support of each individual SCC is essentially incorporating such parallelized linear algebra implementations into the simulation process instead of their sequential implementation. Of course, the reality is that practical application is always complicated in large and complicated runtime systems such as the one for OpenModelica. Therefore, there is always a need for a proper adaptation of the runtime system to utilize such parallelized methods.

Parallelization across Connected Components Equation systems generated from models of physical systems sometimes contain decouplings. That is, some parts of the system might be numerically isolated from the rest. This can happen as a result of the actual model being made up multiple disconnected components, which is rare. It can also happen as a result of certain symbolic processing and causalization operations performed by the modelling tools. Decouplings in the equation system can also be introduced by specific modelling techniques to allow for better parallelization opportunities.

This approach provides a quick and, in some specific cases, flexible parallelization. This approach is one of the directions investigated in this work and is discussed in detail in Chapter 5.

Parallelization across Strongly Connected Component Another parallelization approach is one based on analysing and processing the strongly connected components of the equation system. This approach has been investigated by a few works. [7] has investigated a task graph based parallelization. It utilized task clustering techniques similar to those presented in this work to reduce the complexity of the graph. It was based on static cost estimation done by analysing the structure of the equation system at compile-time. Furthermore, that approach focused on the parallelizing the expressions within each equation (instead of the equations themselves) and was targeted for simulations using distributed memory architectures using MPI. The approach was later extended with pipelining support in [73].

[86] [87] investigated a prototype implementation of parallelization based on the work of [7] but targeting GPGPU architectures. That approach used a prototype Runge-Kutta solver to perform parallelized simulations on CUDA enabled NVIDIA GPUs. 
Casella[19] proposed an algorithm for extracting parallelism from equation systems based on the Block Lower Triangular transformations of the incidence matrices for equation systems. That approach was investigated further and implemented in [102] and then extended in [103]. They employed a semi-static form of cost estimation. While the scheduling operations are still performed at compile time, they utilize profiling results from previous simulations to make scheduling decisions for the current simulation. This can work acceptably for rather static simulations, however it provides no way of responding to dynamic behaviour within a single simulation and is not portable. Furthermore, they seem to have associated communication considerations even though the executions are performed on shared memory architectures. To our knowledge, at the moment of writing, this implementation is the only currently available and usable parallelization apart from this work.

[28] also investigated and implemented an approach based for extracting parallelism by partitioning the Block Lower Triangular structure matrices of equation systems similar to [19].

Parallelization based on analysis of strongly connected components is one of the main focuses of our work and is discussed in detail in Chapter 6. The aim here is to have a parallelization approach and implementation that has no restrictions on model structure or components, no restrictions or preferences on solvers to be used and no requirement for previous simulations. In addition, it has to be capable of adaptive runtime scheduling and should be a stand-alone, flexible and portable implementation to simplify the whole process of integrating parallelization capabilities into existing tools and compilers. 



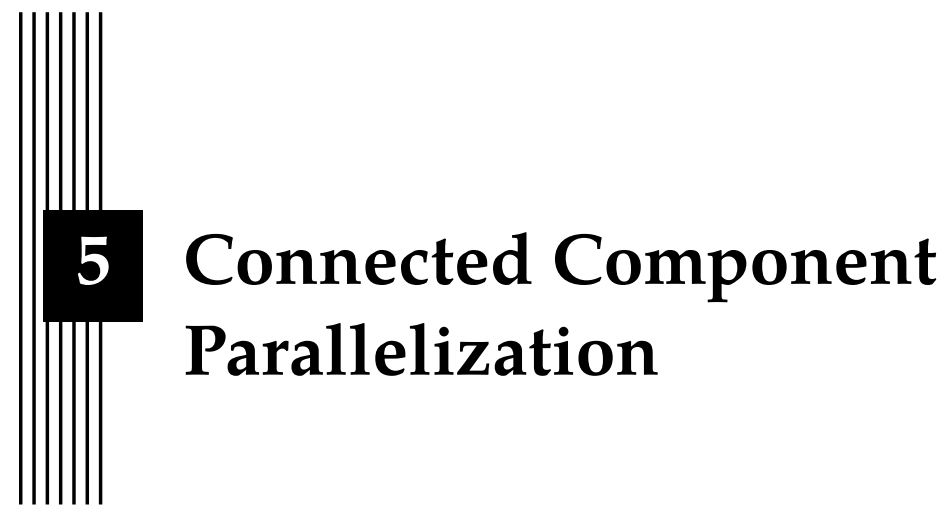

The first parallelization approach taken in this work is based on connected components. This is a relatively direct approach only concerned with the independent set of connected components resulting from the matching and sorting of equation systems. Each set of decoupled components can be executed independently form each other allowing for parallel evaluations. A simple example demonstrating this is shown in Figure 5.1. This is the matched and sorted equation system of the simple RLC circuit discussed in Section 4.2. There are three sets of connected components that can be freely moved and independently executed. These subsystems can be evaluated in the order shown in the right hand figure of Figure $5.1 \mathrm{~b}$ opening the possibility for a balanced parallel execution.

Implementing such parallelism, however, requires consideration in a number of aspects. First of all the complete system needs to be broken down to such decoupled systems. This information can be expected to be provided by the simulation tools that performed the symbolic processing. For example the OpenModelica compiler readily provides that information. Second, if it is to be an effective parallel evaluation, it requires some sort of load balancing mechanism. Third, and perhaps more complicated, is that it requires a thread-safe runtime system with proper memory and thread management. This was not available for the OpenModelica simulation runtime system.

This chapter discusses the approaches and methods taken to introduce a safe and efficient parallelization based on connected components. In addition, it presents approaches that can be taken to improve the availability of 
Figure 5.1: Simple Connected Component Balancing
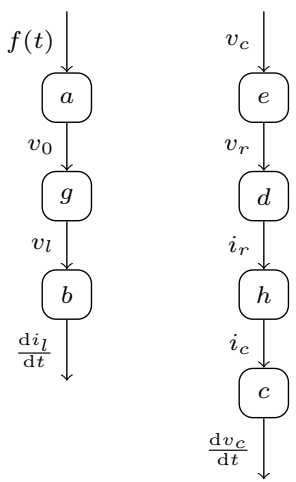

(a)

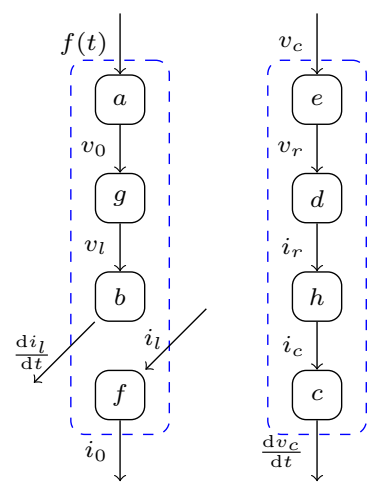

(b)

disconnected components and presents results from the approach and implementation.

\subsection{Integrated Approach}

Before going into the details of the approach it should be mentioned that this parallelization employed an integrated approach. That is, most of the processing and implementation is implemented directly inside the OpenModelica Compiler or in the OpenModelica simulation runtime system. Not all aspects of the parallelization needed to be so tightly integrated, but this was a lesson learned afterwards. Of course some things need to be part of the existing system. For example supporting a thread safe memory model, naturally, requires unavoidable modifications to the runtime system.

Most of the processing for this approach is done at compile time. In addition, all runtime operations are implemented as part of the default OpenModelica runtime system. Extra code needed for multi-threaded execution is generated directly into the model code as well. This meant the code-generator required a lot of modification. The generated code also becomes more obfuscated. Such consequences of a tightly integrated approach are discussed in detail in the subsequent relevant sections.

\subsection{Cost Estimation and Load Balancing}

The set of connected components resulting from symbolic manipulation of equations are usually of different sizes. For example the simple RLC circuit 
of Figure 5.1 has three connected components of sizes 3,4 and 1. Efficient parallelization of such imbalanced systems of course needs some sort of load balancing.

As mentioned earlier the connected component parallelization approach implemented here is tightly integrated with the compiler. This means that all the processing regarding parallelization is done in the compiler at compiletime. One of the consequences of such compile-time processing is static cost estimation. Balancing of a set of independent systems can be done in a straight forward manner. However, to generate an effective balanced system the execution cost of each component needs to be as accurate as possible. In a compile-time approach this cost information has to be estimated manually.

The majority of equations in a typical model Modelica model - as well as most in general equation systems - are the so called single-assignment equations of the form $\mathrm{x}=\mathrm{f}(\ldots)$. The execution cost of such equations can be estimated by analysing the expressions forming the right-hand-side, i.e., $f(\ldots)$. Since we are interested in balancing only, it is not necessary that the estimations are accurate with regard to actual execution time as long as they are consistently scaled from expression to expression.

It is possible for the compiler to traverse the right-hand-side of each simple equation and accumulate the cost by summing up the cost of each subexpression. However, we opted to skip this for performance reasons and instead decided to assign a constant cost for such single-assignment equations in general. That means such equations can be assigned a constant cost value, say $c$. A connected component made up of $n$ such equations will then have an estimated cost of $n \cdot c$.

Such static estimated cost approach was good enough for most cases. However, there are a number of aspects where it can be imprecise. Function calls, for example, cannot be estimated effectively without actually inspecting the statements making up the function body.

Even a complete estimation, based on analysing each statement and expression, cannot estimate all components of complicated systems. For example, external function calls or loops whose iterations depend on variables cannot be estimated properly. Even more importantly there is no satisfactory way of estimating the cost of linear and non-linear systems of equations. For example a linear-system of 10 simple equations will take considerably more computation time than a normal set of 10 equation systems. Non-linear systems - as well as iterative solvers for linear systems - can complicate things further since their execution cost depend on the actual values of the variables affects the convergence rate leading to varying execution times during simulation time.

Despite all of these issues, static-cost estimation and load balancing can be effective for equation systems that are mostly made up of single-assignment equations. It is very fast and has no overhead on the actual simulation of the model since it is all done at compile-time. The strongly-connected par- 
allelization approach discussed in Chapter 6, Chapter 8 and Chapter 7 used a dynamic cost estimation based on runtime measurements to address these limitations.

\subsection{Memory Management}

The OpenModelica runtime system is quite complicated. It has to provide support for simulations which can use different solvers, interactive simulations, external features like FMI etc. It has to support different mathematical operations, optimization features and so on. It has to provide different interfaces for specific purposes, for e.g. Java, FORTRAN interfaces. Support for MetaModelica is also part of the runtime system.

To perform or provide these functionalities efficiently the runtime system needs to have a flexible and efficient memory management system. Among many things this should include support for smart arrays. Smart, in the sense that, the array representations should be aware of the number of dimensions as well as the sizes of each dimension. Since the run-time system is mainly implemented using $C$ this is not available out of the box. This means that there needs to be a custom array container implementation that can work hand in hand with the memory management system. The OpenModelica runtime achieved this by representing Modelica arrays as $C$ structures with additional information about the array. The actual data of each array is located in a global memory pool. The global memory pool operates in a very similar way to a traditional stack implementation of last in first out. Each array structure has a pointer pointing to its own specific data.

Such kind of custom memory management have its disadvantages. For one thing it is adding extra complication to the whole system. Memory management becomes one thing that the development has to additionally consider and maintain. Secondly, custom memory management is a recipe for all kinds of elusive bugs and inconvenience. Therefore it is in some ways counter-productive to use such implementation when there is a widely used and almost guaranteed correct memory management provided by the $\mathrm{C}$ runtime system. However, there were two main motivations for using a global memory pool instead of raw allocations - using malloc, free and their variants - provided by the $\mathrm{C}$ runtime.

The first reason is that using a global memory pool the runtime system can avoid potential memory leaks which can be very troublesome for simulations which take relatively longer time. Simulations involve computations over a range of time steps. That means a small memory leak can build up over time to a considerable amount. By using a custom memory pool the run-time achieves a better and safer memory model.

The second reason is more related to the nature of physical models, specifically which are usually implemented with Modelica. Most Modelica models 
(at least so far) do not have large arrays. This means the amount of memory allocated per array is relatively small, in other words memory allocated per malloc call is relatively smaller. Now keeping in mind that a certain array allocation will most probably be done on every time step, it would be expensive to call malloc at each time step compared to the actual operations performed on the array elements.

This custom global pool based memory management has since been replaced, by default, with a garbage collected memory management that uses the Boehm-Demers-Weiser conservative mark-and-sweep garbage collector [14] [15]. Nonetheless, it was the memory management used at the time of this specific automatic parallelization approach. This meant that the memory model needed to be implemented with consideration for multiple threads. That is, it had to be thread safe. There were two alternatives considered in making the memory model thread safe. These approaches are described here.

\subsubsection{Shared Global Memory Pool}

The first alternative to achieving a thread safe memory structure is to keep the global memory pool and make all allocations atomic. All threads will have access to a common global memory pool that is once. If a thread needs to allocate some memory then it will use the pool's allocation interface which performs allocation protected by a mutex as shown in Listing 5.1.

\section{Listing 5.1: Shared global pool allocations}

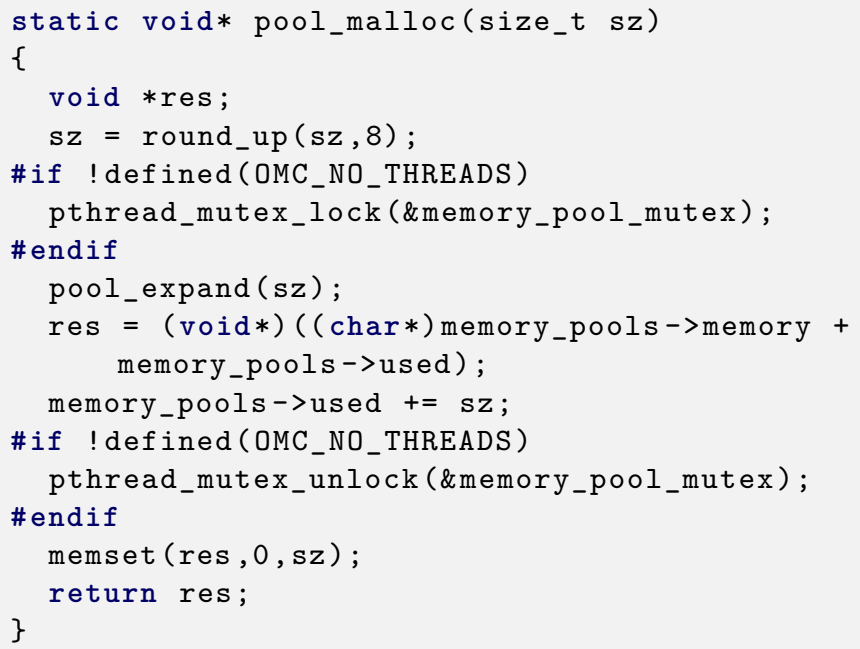


This shared global pool is allows for a quick and easy model that allows concurrent allocations. Of course having lock protected allocations means that there is a potential that multiple threads try to allocate the memory at the same instant and will have to block and wait. This can be a considerable performance bottleneck in multi-threaded executions where the threads have similar operations to perform. For example, when parallelizing a typical data parallel loop where threads more or less execute the same set of instructions reaching the same position at similar points in time. In this case, however, the operations performed by each individual thread are more diverse leading to a relatively non-uniform execution flow. This means, in general, the amount of blocking encountered when allocating memory is relatively less of a performance bottleneck compared to typical data parallel operations.

Although the shared global memory pool is quite convenient and easy to integrate, it has one major drawback. That is, freeing up of memory can only be done at specific execution boundaries. The original single memory pool intended for sequential executions was used similarly to a typical stack frame used by other compilers such as GCC. On every entry to a function the state of the global memory is fetched and saved. Just before the function returns it restores the saved memory state essentially freeing up any allocations done by itself or its callees (if for some reason they have not cleaned up everything themselves). In the multi-threaded operation this cannot be applied since allocations between multiple threads can be interleaved. If a function scope saving and restoring is done then one thread might inadvertently clean up memory used by another thread that performed allocations after the first thread entered its current function.

Memory freeing operations can only be done at points where it is guaranteed that all threads no longer need any memory that they have so far allocated. In simulations of equation systems this is guaranteed at the end of each time step, i.e., one complete evaluation of the equation system. The memory state can be restored at such points preventing any possible leaks from propagating and accumulating over multiple time-steps while still being thread-safe.

\subsubsection{Thread Local Memory Pool}

Note that the memory pool is used only in the code generated for the equations of model. The rest of the runtime system does not need to use the global memory pool. In addition, all variables - that is variables of the mathematical model - that are part of the equation system are put in a separate global array. Each equation directly accesses and updates this global array using updated variables and making its results available respectively. As a result the memory pool need only be used for local allocations and computations within each function representing an equation. 
If no variable residing in allocated memory has to be shared then it is possible to have multiple thread pools that are local to each thread involved in the execution. An array of thread local memory pools is kept in a global variable accessible to each thread. At start of simulation each thread initializes its own memory pool out of the available pools based on its identifier. All subsequent allocations and deallocations by the thread are directed to this specific memory pool. A thread will not be allowed to access any other thread's memory pool by the runtime system.

The thread local memory pool allows for per-function-call state saving and restoring just like the original single memory pool implementation. Each thread can freely allocate and deallocate memory on entry and exit from each function since the pools are mutually exclusive. This is the memory management used in this particular parallelization approach.

This approach is efficient and lock free. However, it comes at the cost of a more complicated runtime system compared to the shared global version. Extra considerations need to be made in creating, initializing and keeping track of the memory pool interface since the OpenModelica runtime system needs to be compiled for a wide range of systems. If a specific system does not support the specific thread framework used, e.g. posix threads, or support for parallelization is not needed then the runtime system needs to correctly fall back on a single memory pool use.

Although the thread local memory implementation was used for the parallelization described in this section, it has since been replaced by a shared global memory implementation. The OpenModelica runtime system now uses the Boehm garbage collector by default eliminating the need for internal memory management. For exceptional cases where the garbage collector cannot be used the shared global memory pool is deemed sufficient since it is easier to maintain.

\subsection{Thread Management}

Simulation of mathematical models is complicated. It is not just evaluating the equation systems. There are a number of checks and book-keepings involved in performing a complete, reliable and efficient simulation. Not all of these operations need to, or even can, be parallelized. In this work we are only interested in parallelizing the evaluation of the equation system as discussed in Section 4.3. Evaluation of the equation system is the most time consuming part of a typical simulation. However, it should be noted that this is mostly because a single simulation involves thousands and thousands of evaluations even for short duration simulations (duration as in the time we are interested in simulating the model from-to, not the time taken by the execution).

The simulation of an executable generated from a Modelica model compiled with OpenModelica can be seen as consisting of two components. The 
model specific code generated by the compiler and the simulation runtime system that is responsible for deriving the simulation among other things. The simulation runtime is the portion responsible for the actual "simulation". Whenever it needs to evaluate specific part the equation system it makes a call to a specific function that is registered as a callback function by the generated code. For example if the runtime needs to evaluate the initial equations of the system it calls functionInitialEquations in its callback structure which is bound by the generated code to ModelName_-_ functionInitialEquations.

The most straight forward approach in parallelizing portions of the generated simulation code is to create threads where needed and terminate them immediately after. For example, to parallelize the balanced system of equations such as shown in Figure 5.1, threads can be created in the corresponding callback function. This means the implementation can launch threads when needed, synchronize them as needed, and continue with the sequential execution when it is done. This has the advantage that the runtime system does not need to be concerned about whether parallelization is used or not. All parallelization issues are kept in the generated code. However, this means threads will be created on every callback from the simulation runtime system. Keeping in mind that such callbacks can be done thousands and thousands of times, launching and joining threads is such a manner will be a huge performance degradation.

An alternative taken in this implementation is to modify the runtime system instead to have a more persistent thread management. The simulation runtime creates threads once. These threads are guided through most of the call tree of the execution. Most of the operations are performed by a single thread while all other threads fall through until needed. When a portion of the generated code that requires multiple threads is reached, the execution assigns the corresponding parts to each threads. It waits for all threads to complete, synchronize, and then continue with a single thread execution while the other threads just fall back until needed. A simplified depiction of this guided execution is shown in Figure 5.2.

\subsubsection{Complexity and Portability Issues}

This approach of creating threads at the start of the main simulation loop and guiding them throughout the execution avoids the extra overhead of creating threads at every evaluation. However, it comes with a considerable cost. First of all the simulation runtime is now complicated by all kinds of multithreading related modifications which makes the code harder to understand and maintain. Every developer needs to make sure that any additional code added to the system is evaluated by a single thread. Which seems okay for someone who is concerned with parallelization but can be quite frustrating for developers who are continuously trying to improve the simulation runtime by adding, modifying or removing features. The fact that some develop- 


\section{Figure 5.2: Simplified thread guidance through runtime system.}

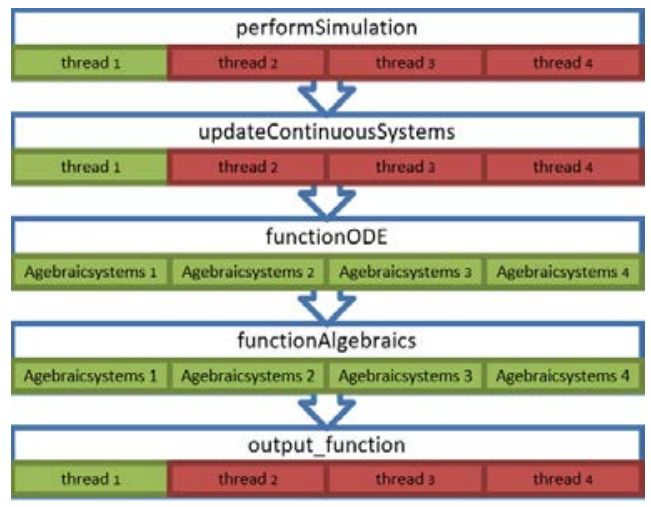

ers are not familiar with multi-threaded programming makes this even more of a concern.

In addition, there is now an extra consideration of portability. The runtime system needs to be compiled with parallelization support, e.g. OpenMP. Extra considerations need to be taken in order to make sure the runtime can be compiled on systems that do not have support for the specific parallelization framework. For example, parts of the simulation code needs to be enabled disabled by macros, some multi-threading related library function need to be over-ridden if there is no support for them and so on. Some of these issues were alleviated by moving parts of the simulation runtime code to the generated code. That is, even though the code generated for this part does not really differ from model to model, it is enabled or disabled depending on whether the multi-threading support is needed or not when the model is compiled.

These issues were some of the main motivations in deciding to design and implement a stand-alone library based approach (See Chapter 8) for the strongly connected parallelization discussed in Chapter 6.

\subsection{Improving Decoupling}

The simple circuit example of Figure 4.1 and the corresponding graph of sorted equation system shown in Figure 4.3 might have given the idea that it is common to have such decouplings. Unfortunately this is not the case in most models. Models of physical systems are usually highly connected. If different parts of a model have no relationship with each other, then modellers will not put them in the same model. They will just split them into multiple models. Of course such decouplings can arise from selection of some variables as known variables by the symbolic processing method used. This is exactly 
the case for the circuit model example of Figure 4.3. Sine some variables are selected as state variables, i.e., variables computed by the integration method using the values of their derivatives, they introduce some decoupling into the system. This decoupling is of course limited to one evaluation. Successive evaluations still need the updated values from the integrators for these state variables. So in a sense, all the variables are connected over the simulation but the system has some decoupling within each evaluation. Since the parallelization is concerned with each evaluation individually, we are able to evaluate the system using multiple threads.

It is not common to have a big enough set of decoupled components in a given model to have a balanced parallel execution. There are usually a few sets of connected components that are composed of a very small number of equations while the majority of the system remains connected. The decoupling of equation systems can be improved by using delayed systems. Instead of having equations depend on variables from the same time step, we can introduce delays to some equations essentially making them dependent on previous values of a given variable.

A given physical system can modelled in such a way that it is divided into multiple sections that model the propagation of physical quantities of interest over the system. This approach is used in the modelling technique called Transmission Line Modelling (TLM). The TLM approach is a widely used technique in modelling to allow for distributed simulation where different parts of a model can be evaluated using multiple solvers instead of one centralized solver [98]. Distributed solver approaches use multiple solvers to solve different parts of the equation system instead of using one centralized solver. This opens up the opportunity for a parallelization over time [91]. Techniques such as pipelining and inlining distributed solvers can also be used to improve performance [72] [lundvall2008automatic]. There are also modelling tools based on the TLM techniques [30] [69] [96]. Uses of TLM techniques in different physical domains are demonstrated in [18] [68]. There is also an ongoing work with the OpenModelica effort to provide a generalized simulation framework based on TLM-based and FMI co-simulation interfaces [84].

\section{Listing 5.2: Delays in Modelica model}

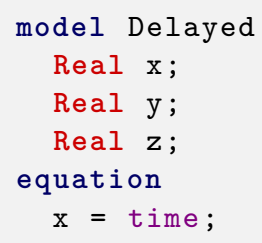




\section{Figure 5.3: Delayed variable dependencies}
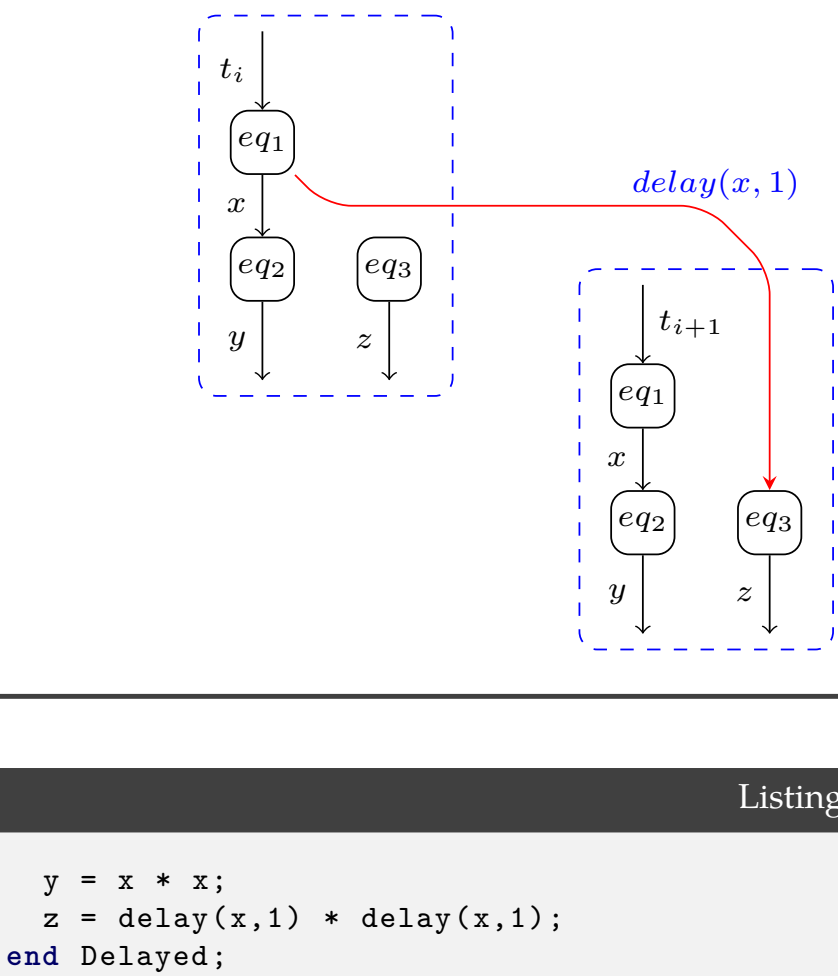

For models implemented in the Modelica language, the delay $(\mathrm{x}, \mathrm{T})$ operator can be used to represent such delays. This operator provides the value of the variable $\mathrm{x}$ at some previous time $\mathrm{t}-\mathrm{T}$ where $\mathrm{t}$ is the current simulation time instant. Listing 5.2 demonstrates a very simple use of delays in a Modelica model. The variable $y$, which is computed using the second equation, depends on the value of $\mathrm{x}$. That means the first equation needs to be computed before the second one within each time step. The variable $z$, on the other hand, does not have such a dependency. At each time step $z$ can be computed using the third equation simultaneously without depending on the first equation since $z$ only depends on previous values of $x$ which are already computed by preceding evaluations. These dependency relations can be seen better in the visualization of successive evaluations of the equation system shown in Figure 5.3.

Introducing delays has essentially replaced the in-step dependency of $z$ on $\mathrm{x}$ by an over-step dependency allowing the parallelized computation of part of the equation system within each evaluation. The results after simulation 


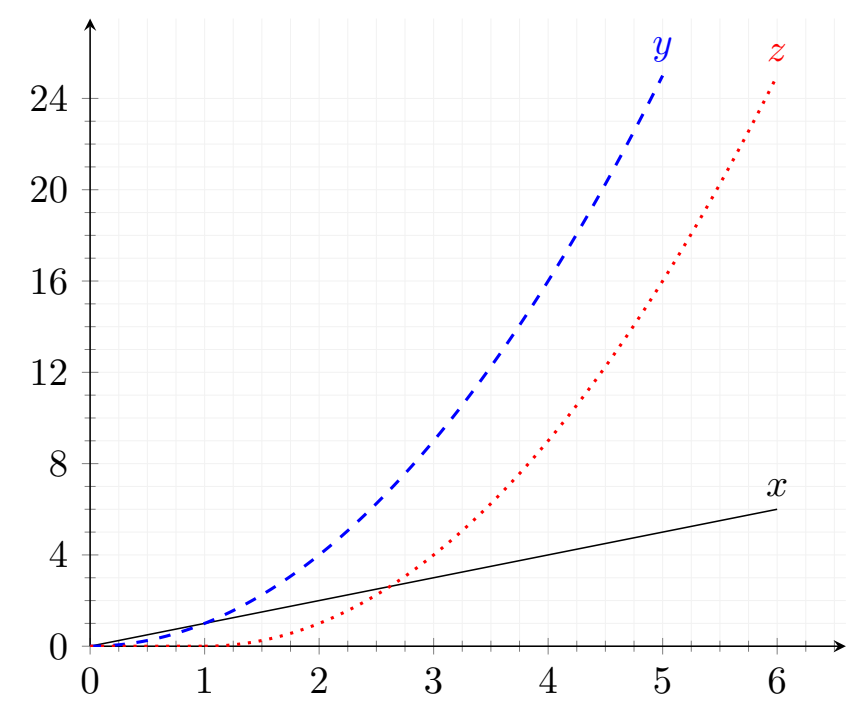

Figure 5.4: Delayed variable solution trajectories

of this model is shown in Figure 5.4. As expected the value of $z$ at time $t$ is the same as the value of $y$ at time $(t-1)$ since we have a one second delay. For time points less than the delay, i.e. 1 second here, the value of the variable $z$ is zero (which is the default start value given to the variable).

This approach of introducing decoupling by using modelling techniques such as TLM implemented with delayed values can work well for specific domains or problems. However, it is not generally applicable. Even when applicable or suitable, the approach requires, with regard to parallelization, pre-planning and specific form of modelling. It remains not applicable to the wide range of component models that are already available. Furthermore, the use of delays might require specific solver feature such as limiting the maximum step size. If the step sizes taken by the solver used is greater than the minimum delay time in the model, there might be a need to interpolate the value at that exact point from the points at the time steps taken by the integrator. This interpolation can also happen when solver step time points do not coincide with delay time points.

\subsection{Case Study}

To be able to evaluate the relative performance gains of the implementation we have used a simple hydraulic system model consisting of a volume with 


\section{Figure 5.5: A volume with a pressure relief valve}

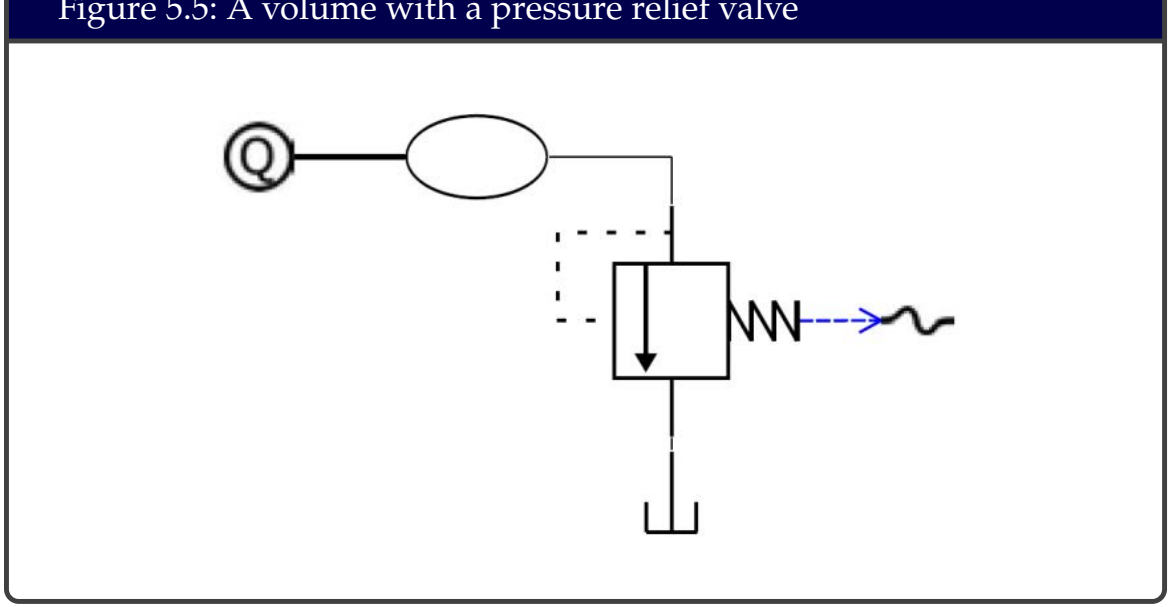

a pressure relief valve as shown in Figure 5.5. The volume is modelled as a transmission line. It is divided into a number of segments with orifices that are connected to each other. In practice this means that each individual volume segment will receive values for pressure and flow from its neighbouring components with some delays.

A pressure relief valve is a safety component, with a spring at one end of the spool and the upstream pressure, that is, the pressure at the side of the component where the flow is into the component, acting on the other end. The pre-load of the spring will make sure that the valve is closed until the upstream pressure reaches a certain level, when the force from the pressure exceeds that of the spring. The valve then opens, reducing the pressure to protect the system.

Simulations are performed on models where the volume is split into different number of segments. All simulations are performed with the OpenModelica compiler. However, instead of the default solver which is DASSL, the simulations use an explicit $4^{\text {th }}$ order Runge-Kutta solver. DASSL is a variable step solver (See Section 15.2.5) which means the step size taken is adaptive to the behaviour of the system around the current evaluation. The OpenModelica compiler, at the time, did not provide the option to limit the maximum step size that can be taken by the DASSL integrator. This means it might be necessary to interpolate some values if the step sizes are larger than or do not much the delay times in the model. Therefore, a $4^{\text {th }}$ order Runge-Kutta solver with step size of $5 * 10^{6}$ is used instead.

The results of performance measurements with the volume split into different number of segments Figure Figure 5.6. The measurement does not include the model instantiation, flattening and back-end specific operations. Only the computation time for the simulation executable to complete is mea- 


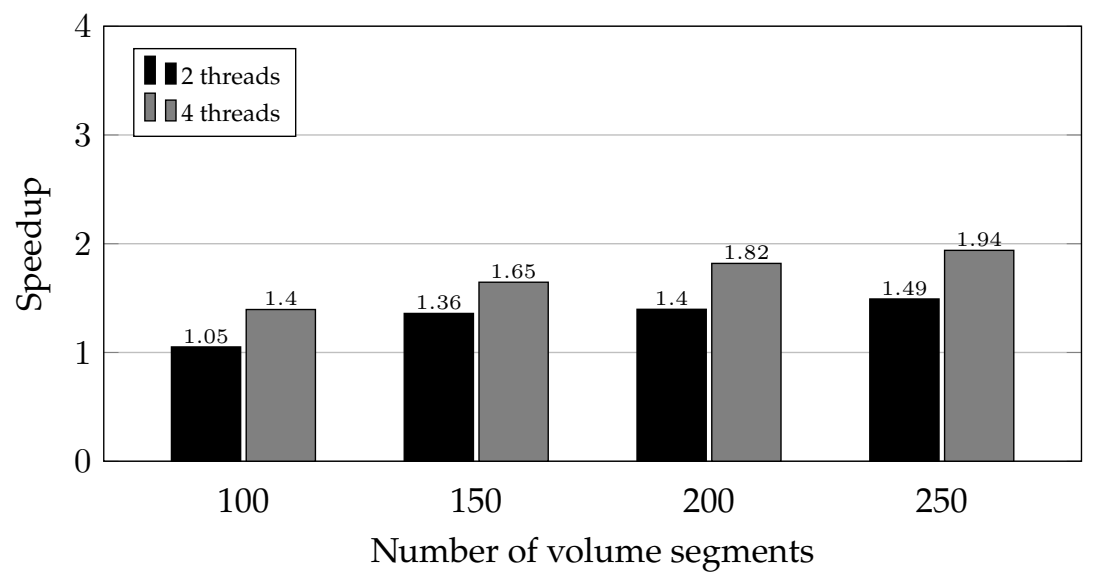

Figure 5.6: Speed-up for different number of segments

sured and compared. The simulation is performed on an Intel®Xeon W3565 quad-core CPU @3.2GHz.

\subsection{Conclusions}

The connected component based parallelization approach discussed in this chapter has its advantages. It can provide acceptable performance improvements as demonstrated by the hydraulic system test case presented. Most of the processing needed for the parallelization is done compile time, which meant that the actual simulation had less overhead and complication. Static cost estimations can be expected to be effective for models that have regular structures leading to a balanced parallel execution with little discrepancies.

The memory management considerations and improvements are perhaps the more complicated parts of this approach and implementation. However, those issues are not specific to the approach. The problem was that the target runtime system's memory management was not thread safe to begin with. The thread local memory design allowed for a lock free and flexible memory system that can be used. This applied not just for this approach, but for any parallelization approach in general, until the OpenModelica system eventually moved to a garbage collected memory system. In the end the thread local memory pool was replaced by the shared global lock based memory management discussed in Section 5.3.1 to reduce the overall complexity and to allow seamless switching between the garbage collected and shared global pool.

Regarding thread management, the approach managed to have low overhead by removing the need to launch and join threads multiple times. The 
launch-once and guide approach turned out to be effective in terms of efficiency although it led to increased complexity of the runtime system.

The approach is not free of shortcomings however. In fact, it has a number of serious limitations. First of all it is heavily dependent on the fact that there are enough set of decoupled systems in the model. A single model, naturally, seems to contain highly dependent structures. Modellers simply do not put multiple unrelated components in a single model. Although such decouplings can appear due to symbolic processing decisions such as state selection, they are limited in most typical cases.

Certain modelling techniques can be used to improve the decouplings as discussed in Section 5.5. However, this is unappealing for two reasons. One, the physical system the model represents should be suitable for such techniques. It is not a solution that can be applied to all systems in general. Second, such techniques, e.g. using the transmission line approach, require that the model be either originally written with such considerations in mind or they have to be modified later just for the sake of parallelization. This makes the models more obscure. Even if it is acceptable that the models become more obscure, it still needs extra considerations and modifications which defeats the purpose of automatic parallelization to some extent.

The fact that cost estimation is done at compile-time meant that there are a number of structures that it cannot satisfactorily estimate. Performing load balancing once at compile-time also means that the simulation has no way of responding to changes in system behaviour at simulation time.

The last problem is more with the implementation than the approach. Most of the implementation and processing was tightly integrated into the compiler and the runtime system. This added a certain amount of complexity to the environment. If more complicated parallelization related operations and features are added in a similar manner to the compiler, it will quickly become more difficult to maintain and improve. In an active development environment such as the OpenModelica system it is necessary that there is a modular implementation approach where features should not be implemented directly into the normal operation sequence. This will make life easier for the developers as well as simplifying the maintenance and improvement of the feature separately from the rest of the system.

The strongly connected component parallelization approach that is discussed in the next chapter has tried to address all of this problems. 



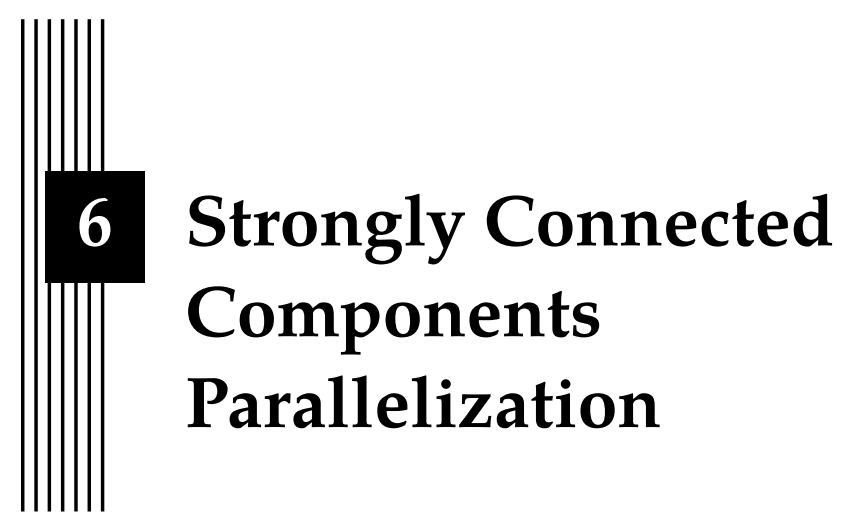

A parallelization approach based solely on connected components can have a number of disadvantages as we have seen. Real life physical problems do not usually provide us with enough sets of connected components to have enough parallelism. Modellers simply do not put disconnected components into their models. They will make two models of it instead. Even if such decoupled systems could arise due to mathematical properties of an otherwise connected system as we have in the examples of the previous chapter, they are usually limited. Explicitly introducing decoupled systems into the model formulation using methods such as Transmission Line Modelling based on delays is also not desirable since it would defeat the purpose of fully automatic parallelization be requiring modifications to models. In addition, it can make the models more obscure and unintuitive.

In this and the next few chapters we explore a parallelization approach based on manipulating strongly connected components. Any directed graph has at least the same number of strongly connected components as connected components. In reality, for equation systems resulting from complex models of equation systems, there are by far more SCC than $\mathrm{CCs}^{1}$. That means we can expect a much better detection and utilization of parallelism if we are working with SCCs. However, nothing comes for free. There are a number of complexities and issues that will arise when we move to SCC based paral-

\footnotetext{
${ }^{1}$ Note that strongly connected component is the maximal set of components that are reachable from each other. Since any vertex (that is not part of a bigger SCC) is reachable from itself it is an SCC by definition.
} 


\section{Figure 6.1: A simple electrical circuit}

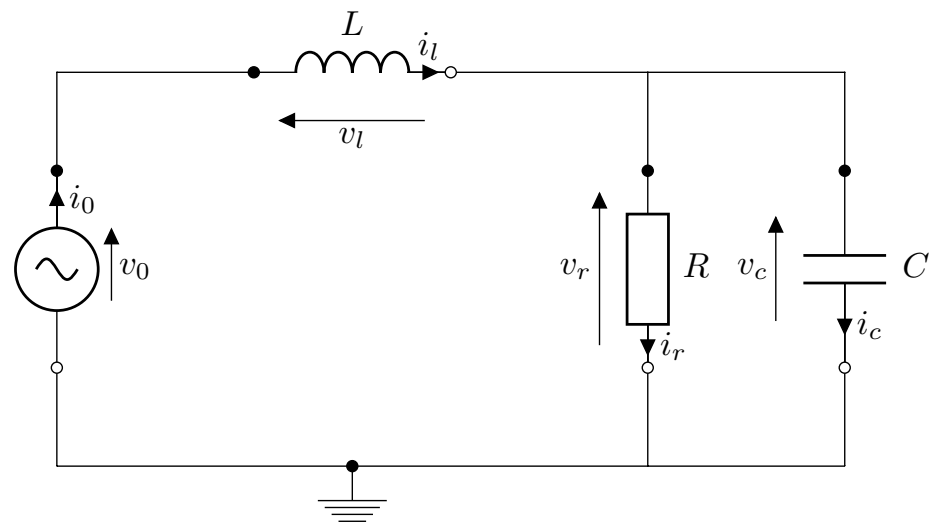

lelization. Considerations regarding what should be expected an accounted for a correct and efficient automatic parallelization of complex equation systems will be discussed here.

This chapter gives a general overview of the strongly connected parallelization approach. Aspects of the approach such as the need for proper scheduling and clustering, data dependency issues, memory and thread management related considerations are introduced here. The next chapter, Chapter 7 , presents the scheduling and clustering methods used in the work in more detail. Chapter 8 introduces the accompanying task system parallelization library whereas performance evaluations of the approach are presented in Chapter 9.

\subsection{Equation System Structure}

We will start with a simple example of RLC circuit to demonstrate how even the same model formulated in equivalent but different ways can have a considerable effect on the structure of the resulting system with regard to parallelization.

Consider the simple RLC circuit shown in Figure 6.1. This is exactly the same circuit as Figure 4.1 except we now have connected the resistor to the inductor and the capacitor to the resistor instead. As far as the laws of electrical circuits are concerned this is exactly the same circuit and should behave the same. If we list the equations of the circuit as we did previously we get the system of equations of Equation (6.1). 


$$
\begin{aligned}
v_{0}=f(t) & \equiv & v_{0}-f(t)=0 \\
v_{l}=L \cdot \frac{\mathrm{d} i_{l}}{\mathrm{~d} t} & \equiv & v_{l}-L \cdot \frac{\mathrm{d} i_{l}}{\mathrm{~d} t}=0 \\
i_{c}=C \cdot \frac{\mathrm{d} v_{c}}{\mathrm{~d} t} & \equiv & i_{c}-C \cdot \frac{\mathrm{d} v_{c}}{\mathrm{~d} t}=0 \\
v_{r}=R \cdot i_{r} & \equiv & v_{r}-R \cdot i_{r}=0 \\
v_{c}=v_{r} & \equiv & v_{c}-v_{r}=0 \\
i_{0}=i_{l} & \equiv & i_{0}-i_{l}=0 \\
v_{0}=v_{l}+v_{r} & \equiv & v_{0}-v_{l}-v_{r}=0 \\
i_{l}=i_{r}+i_{c} & \equiv & i_{l}-i_{r}-i_{c}=0
\end{aligned}
$$

This equation system is equivalent to that of Equation (4.8) but formulated in a slightly different way. That is Equation (6.1g) now uses $v_{0}, v_{l}$ and $v_{r}$ whereas Equation $(4.8 \mathrm{~g})$ used $v_{0}, v_{l}$ and $v_{c}$. the two formulations are mathematically equivalent since $v_{c}$ is equal to $v_{r}$ by Equation (6.1e). Modelling tools that generate equations based on a drag-and-drop and connect approach, such as graphical modelling with Modelica tools, generate equations based on the order of connections. Therefore, it is quite possible that they generate this system instead of Equation (4.8).

We can follow the same matching procedure as we did for the previous formulation to generate a causalized equation system for this circuit. If we represent the system as a bipartite graph, perform the matching process and then orient the graph as we did for Figure 6.1, we will arrive at the directed graph of Figure 6.2.

Although we started with a mathematically equivalent set of equations this time we ended up with two sets of connected components instead of three as was the case for Figure 4.3. A connected component parallelization approach can now only run these two CCs in parallel. Assuming that we have two processing units and each node takes a unit time to compute on any of these computational units, the effective ideal speedup that can be obtained is now reduced to 1.17. This a considerable reduction compared to the ideal speedup of 2 that can be achieved for Figure 4.3 for the same model.

\subsection{The Need for Scheduling}

In parallelization based on connected components, discussed in Chapter 5, we were dealing with independent (disconnected) sets of equations. This means there was no ordering or scheduling needed between each set of connected components. Of course, the equations forming a single connected 


\section{Figure 6.2: Directed Graph of matched RLC circuit equations}
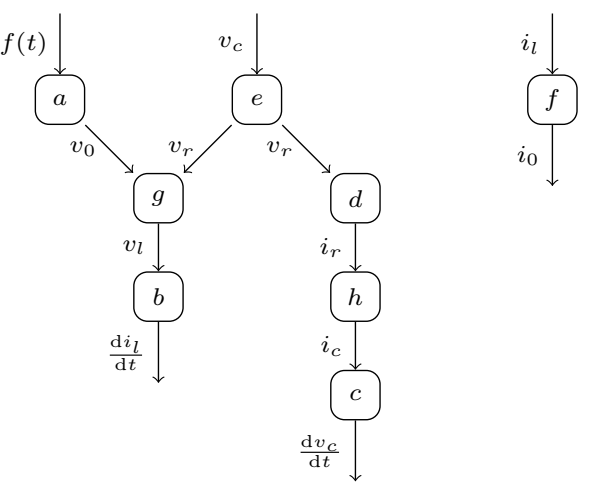

component needed to be evaluated in a specific order. This can be achieved with a simple topological sorting similar to sequential evaluation. Sets of equations grouped as strongly connected components, on the other hand, have the possibility of having connections or dependencies between each set. These dependencies need to be obeyed strictly to have a correct evaluation.

The simple RLC circuit example of Figure 6.2 has eight strongly connected components - each node is an SCC by itself if it is not part of a bigger SCC and two connected components. A connected component approach here will result in a severely unbalanced evaluation where one set takes seven times as much to complete compared to the second set, assuming each equation takes a unit amount of time to evaluate. If instead each SCC is considered by its own the evaluation can still be performed in 4 time units. However, it is necessary that the order of execution does not violate the dependencies between each equation. This means there is a need to have some form of scheduling and synchronization mechanisms in place.

Most real-life models are a lot bigger and much more complicated. They contain hundreds and thousands equations ranging from simple assignments to large linear and non-linear systems. They vary hugely in structure and behaviour. Therefore, it is vital that the scheduling and synchronization mechanism be efficient and flexible. The scheduling and synchronization mechanisms used and implemented in this work are discussed in detail in Chapter 7.

\subsection{Data Dependencies}

The edges in the directed graphs of matched and sorted equation systems such as Figure 6.1 signify data dependencies due to variables usage. If a variable defined/assigned to by equation $i$ is used in equation $j$ then there will 
be a directed edge from $i$ to $j$. All the parallelization approaches used in this work are aimed at shared memory architectures. This means there is no communication cost between evaluations of different equations or tasks. That is, the edges signify only dependencies or orders of execution.

In general, data dependencies in program statements are classified into three categories: Anti-dependencies, Output dependencies and True dependencies. Out of these three types only the true dependency is relevant for an equation system generated from a valid Modelica model. The reason for this behaviour is as follows. First of all the matching of equation systems (See Section 4.2.1) decides which equation is going to be used to solve for each variable. Since the matching is one-to-one, there is no possibility of two assignment forms of these equations solving for the same variable. This eliminates any possibility of output dependencies in the correctly matched systems. The fact that there is only a one-to-one dependency between variables and equations also means that, as long as true dependencies are detected and obeyed, there cannot be any anti-dependencies since that would mean there is a second equation defining a given variable. ${ }^{2}$

The Modelica language has numerous equation structures. They vary from the ubiquitous equality equations of the form $f(\mathbf{x})=g(\mathbf{y})$ to

- array equations

- if equations

- when equations.

- algorithm sections

- $\ldots$

These are integral parts of Modelica language and what makes it so suitable for modelling systems across many domains and applications. As they say one man's meat is another man's poison, this flexibility and ease of use for the modellers comes at increased complexity for the compilers/developers who need to extract each dependency correctly.

Dependency analysis of large equation systems is a difficult task due to these issues and also the added extra structures and representations that the compilers use internally to represent these systems of equations. Incorrect simulations results or downright terminated simulations due sanity check failures are an incredibly difficult task to debug, localize and identify. In fact, even at the moment of this writing there are a few models that fail to simulate with parallelization due to possible issues with missed dependencies. The dependency specification and analysis related implementation in the ParModAuto task system library are discussed in detail in Section 8.3.3.

\footnotetext{
${ }^{2}$ This discussion also holds for systems of linear and non-linear equations. Even though these coupled systems define/compute more than one variable as a whole, all the variables solved in that system are not defined by any other simple or system of equation.
} 


\subsection{The Need for Clustering}

Equation system representing large and complex physical systems contain hundreds and even tens of thousands of equations. For Modelica, for example, there are a whole range of these fairly large models from multiple libraries being used by modellers on a day to day basis. While the number of equations can be so large, each individual equation is relatively simple in most cases. Even with a very efficient scheduling approach, attempting to have a parallelized evaluation of such large models will require a disproportionate amount of book-keeping and tracking relative to the cost of evaluating each of the equations.

It is vital that the complexity of such large systems is reduced to an acceptable size to offset the overhead of having to keep track of a large number of small operations. Of course, these complexity reductions should be made in a manner that does not unnecessary limit the amount of potential parallelism that was available in the original system.

Equation system of most physical systems also contain a wide range of strongly connected components in terms of size. Most equations in a typical model are single assignment equations such as all those in the RLC circuit example of Figure 6.2. However, as the systems grow in size and complication, inevitably, they are bound to have a number of equation systems that cannot be strictly converted to single assignment forms due to cyclic dependencies - also known as algebraic loops in modeling and simulation literature - leading to linear or non-linear systems of equations. These linear and non-linear systems of equations vary in size from a handful of equations to hundreds. They need to be solved as a whole using linear algebra methods such as Gaussian Elimination or Newton's method. These linear algebra operations are relatively a lot more expensive than most other equation structures that can appear in the system. It is necessary that the overall execution is balanced around these large tasks to have an efficient evaluation.

There are number of clustering heuristics used in this work to reduce the overall complexity of the task graphs for such large equation system containing multiple linear and non-linear systems. In addition to reducing the overall complexity, these heuristics also try to produce a balanced system to assist with scheduling efficiency. In addition they also try to improve data locality. These are discussed in detail in Section 7.3.

\subsection{Stand Alone Implementation}

In addition to the limitations incurred by dealing with connected components, the previous approach also suffered from the integrated approach it took towards processing and parallelization. Implementing parallelization related mechanisms and improvements as an incorporated part of the compiler added to the complexity. This extra complication can be more prob- 
lematic in an actively developed software like OpenModelica. There are a number of developers who are simultaneously working on the compiler and related tools. Most of them are not concerned with parallelization.

The code generation and runtime system is complicated as is. Adding multi-threaded code generation into it means that changes that would otherwise be straightforward can sometimes be a lot more complicated to apply. In addition, it also meant that the correct operation of multi-threaded execution can be easily broken by changes that did not account for it.

In order to address these issues the parallelization approach discussed here opts for a stand-alone library based implementation. This approach considerably reduced the amount of extra code that is introduced to the compiler, code generator, and runtime system. In simple terms it is now as easy as just replacing a single function call in the generated code and linking to the parallelization library, ParModAuto. The stand-alone implementation, the motivations behind it, the considerations taken in designing it, and its overall operations are discussed in detail in Chapter 8.

\subsection{Memory and Thread Management}

The initial design and implementation of this SCC based parallelization used a shared global memory pool model discussed in Section 5.3.1. As mentioned in the discussion, cleaning up and reclaiming of pool memory can only be done at points where there is a guarantee that all threads no longer need their allocated portions. For large models that can perform a large amount of array allocations, this can be problematic since they can accumulate a large amount of memory before the next cleaning stage is reached.

Fortunately, the OpenModelica runtime system eventually moved to full fledged garbage collected memory system using the Boehm-Demers-Weiser (Boehm-GC) garbage collector [14] [15]. The garbage collector has support for multi-threaded operations across multiple platforms. This simplified the implementation to some extent.

As a side note we would like to mention some issues encountered during this approach to demonstrate a few of the many challenges faced when trying to provide a platform and performance portable parallelization framework for active development environments.

[I[

The implementation uses the Intel Threading Building library (TBB) for thread management, the Boost Graph Library (BGL) for graph related primitives, and the Boehm garbage collector since it has to work with the OpenModelica runtime. Most of the development was also mainly done on a Windows machine. The garbage collector needs to know about all threads that need to allocate memory in order to be able to clean-up properly. This can be achieved in two ways. The first and simplest option is to create 
threads using the interface provided by the garbage collector. Essentially, the collector overrides/redirects the necessary thread related functions, e.g. pthread_create() for POSIX and _beginthreadex() on Windows. The second option is to register each thread manually. This approach can be taken if threads cannot be created using the functions provided by the collector. In this case, since the thread creation is delegated to TBB, we had to explicitly register each thread. The TBB system is initialized with a specific number of threads and then they are given dummy tasks in which they register themselves. This worked out okay for relatively shorter and smaller simulations. However, as it happens, TBB actually does not guarantee that the same set of threads will be used for subsequently submitted operations. A new thread can be created at any point and can replace any of the original threads. The guarantee is no more than the number of threads specified at initialization will be used simultaneously. In most operations this is inconsequential. Not for our case, since the garbage collector will not clean from a thread that is not registered already. Unfortunately the error messages from the collector get lost through the chain of OpenModelica runtime functions eventually reporting a completely different error, e.g., dimension mismatch when copying an array. Furthermore, the errors appear at seemingly random points on successive simulations. The first assumption when such assertion failure errors appear is that there is some missed dependency in creation of the task graph. This specific problem lingered for quite some time until it was eventually fixed. The simplest fix was to check and register threads at every invocation of the task system. However, this will incur unnecessary overhead since only one or two extra threads are created in such a manner during a whole simulation. Instead, we decided to modify TBB to use the garbage collector's threading functions and provide this modified version of TBB. This should have been the end of it except that the same errors kept appearing in a similar manner.

TBB is built as a DLL on Windows systems. Since the custom version of the modified TBB used the Boehm GC (built as static library on Windows) it has to link to it. The actual model executable code generated by OpenModelica also uses the garbage collector. Therefore, the model executables also link to the Bohem-GC library. The Boehm-GC implementation uses a global array to keep track of threads that it knows about. This would work just fine if the library is built as a shared library. It also will be of no issues on Linux systems. However, Windows, has a different behaviour. Windows DLLs linked to a static library will incorporate all the static libraries methods and variables as a local instance without making them available to the outside. This means an executable linked to such a DLL while also explicitly linking to the static library will have two copies of the static library independent from each other. The version in the DLL is not visible to the linker so it will pull the explicitly linked static library as well. Since Boehm-GC maintained a list of known threads in a global table, there were two copies of this thread table in the final 
executable. This means threads created by TBB are tracked by its own copy of the GC while the rest of the executable used another copy of GC unaware of the threads created by TBB. In the meantime Linux operations are completely oblivious to this issue since shared libraries expose all definitions by default.

To summarize, once single overlooked extra linking of a static library on Windows systems resulted in a completely unrecognisable issue with symptoms that are normally associated with dependency analysis. While this is an extreme example caused by how the GC was designed and how Windows linking worked, it serves to enforce the fact that a lot of effort in portable parallel programming can be consumed by error detection and debugging instead of actually designing and implementing parallelization algorithms and methods.

1]] 



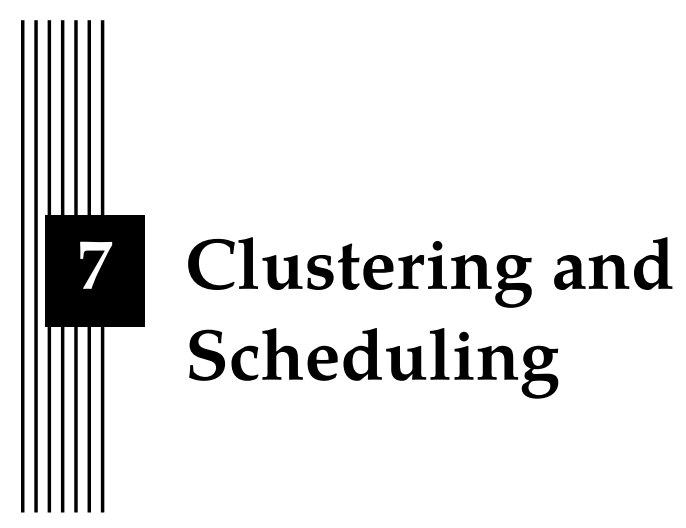

Equation systems generated from a physical system modelled with mathematical modelling languages such as Modelica can have thousands of components. Each strongly connected component is treated as an individual node in the task system representation. If we consider most equation systems models the majority of these SCCs are single component SCC containing simple assignment equations. These simple tasks take a tiny fraction of the simulation time individually compared to multi component SCCs. These multicomponent SCCs contain tens or even hundreds of simple equations forming Linear and Non-linear systems. These systems can be relatively the most time consuming parts of some equation systems and destroy the relative uniformity obtained from the common single assignment equations.

It is possible to directly schedule this large task systems with many small tasks. However, this is not recommended since these small tasks incur more overhead in management and execution than it is gained from running them in parallel. Thread switching and management to execute just one single assignment statements is not feasible. Therefore, it is usually advantageous to reduce the overall complexity of the graph even if that means there will be a loss of potential parallelism.

The implementation provides a few clustering heuristics to address, or in some sense, take advantage of the abundance of these simple tasks. This clustering heuristics aim to reduce the overhead of managing small tasks as well as attempting to improve locality as discussed in Section 7.1. 
The implementation also provides two basic schedulers. The first is the so called Level Scheduler or Wave-front Scheduler. This is an adaptive semi-static scheduler that is based on a step and synchronize mechanism. It is adaptive in the sense that it can potentially generate new schedule - by re-clustering the original task system - between successive evaluations depending on the behaviour of the system. It is also semi-static in the sense that it can dynamically schedule tasks at the same level if there are more tasks than processing units.

The second available scheduler is the so called Flow Graph Scheduler. This scheduler is designed as a wrapper to the Intel TBB Flow Graph (TBBFL) implementation. Intel TBB Flow Graph implements its own a message based work stealing algorithm to dynamically execute tasks in the graph.

Directly applying these schedulers to a task system such as those generated from Modelica models it will not be effective for reasons discussed above. For this reason the schedulers can be, and should be in most cases, associated with one or more of the clustering heuristics available.

\subsection{Task Clustering: Reducing Overhead, Improving Locality, and Balancing}

The task clustering heuristics presented here serve three main purposes.

Reducing Overhead One of the main benefits of clustering large task systems is the reduction in the overall overhead. As mentioned earlier individually treating thousands of tiny tasks is not feasible in terms of efficiency. In an ideal machine there would be no need to cluster the system and we can expect all the potential parallelism to translate to obtained parallelism. Of course real life uses have to deal with overheads in keeping track of all these tiny tasks. In most cases these small tasks cost more to manage than they take to execute. Some of the clustering heuristics presented here try to find a balance between the reduction in overhead and the apparent lose in potential parallelism.

Improving locality Tasks with direct dependency, i.e., parent and child, imply that data updated by one task is used by the other. This two tasks can be merged early to improve temporal data locality possibly improving cache efficiency. For example a node with a single parent can be merged with the parent node to make sure that a variable is used as it is updated. This exact clustering process is provided by the Merge Single Parent (MSP) heuristic in ParModAuto. In addition, these kinds of merging rules reduce the potential of a task being moved further away from its closest tasks due to other clustering rules being applied subsequently. However, care must be taken in 
decisions of merging tasks since these rules can, in some cases lead to cyclic dependencies in the task graph (See Figure 7.5 for example).

Balancing costs Some schedulers benefit from having tasks that take a proportionate amount of time to complete. For example the Level Scheduler mentioned above benefits needs a balanced system of tasks at each level to perform well. The abundance of small simple assignment equation tasks in most Modelica models is advantageous here. These small tasks can be moved around and merged to construct bigger tasks that match the execution cost of large linear or non-linear systems of equations or expensive function calls. These balanced merged tasks can then be assigned to different units and can avoid idling.

\subsection{Background}

The problem of clustering a task graph is not new. It is also not a single solution problem. They type and properties of the clustering to be performed depend on the expected use of the clustered graph. For our purposes, clustering is aimed at three main issues as discussed above, namely reducing general complexity, improving locality, and balancing of costs. These targets can be achieved by clustering heuristics based on simple classical optimization problems. Most of these are unfortunately "hard" problems. Fortunately the requirements of clustering for equation systems is not very strict on tightness. Some core classical problems that are the bases for the clustering heuristics used in this work are introduced below.

\subsubsection{The Bin Packing Problem}

The Bin Packing problem is a classical optimization problem that deals with partitioning a set of items into as few bins as possible while making sure that the total sum of some selected attribute of all items packed into the same bin does not exceed a specified value. The problem can be formally defined as:

Let $C$ be the multiset

$$
C=\left\{c_{1}, c_{2} \ldots, c_{n}\right\}, \quad c_{i} \in \mathbb{R}, 0<c_{i} \leq 1 \quad \forall c_{i} \in C
$$

partition $S$ into a minimum number of $k$ mutually disjoint multisets :

$$
B=\left\{B^{1}, B^{2}, \ldots, B^{k}\right\}, \quad B^{i} \subset C, B^{i} \cap B^{j}=\varnothing
$$

such that

$$
\sum_{c_{i} \in B^{j}} c_{i} \leq 1, \quad \forall B^{j} \in B
$$

The Bin Packing problem is interesting in clustering applications where there are cost considerations of tasks. We are often interested in collecting or 
merging tasks into clusters based on some proximity criteria while making sure that the overall cost of the resulting cluster does not exceed a given cost limit.

Online Bin Packing: Items to be packed arrive one at a time. A decision has to be made on where the current item should be put before considering the next item for any decision.

Offline Bin Packing: In this version of packing the items to be packed are known and available beforehand. This allows for preprocessing the items to be packed to improve the efficiency and accuracy of packing algorithms and heuristics.

Bin packing is a NP-hard problem [47] ${ }^{1}$ [58]. However, there are a number of polynomial time approximation schemes for the problem.

\subsubsection{Polynomial Time Bin Packing Approximations}

There are a number of approximation algorithms proposed, over the past 40 or so years, for the bin packing problem. These algorithms vary in their simplicity, approximation factors, and computational complexity. However, similar to most approximation algorithms for other NP-hard problems, these approximation algorithms are relatively straight forward to implement and analyse compared to exact algorithms.

The Next Fit (NF), First Fit (FF) and Best Fit (BF) are among the simplest approximation schemes for online bin packing. Consider the multiset

$$
C=\left\{c_{1}, c_{2} \ldots, c_{n}\right\}, \quad c_{i} \in \mathbb{R}, 0<c_{i} \leq 1 \quad \forall c_{i} \in C
$$

and an infinite set of bins $B=\left\{B^{1}, B^{2}, \ldots\right\}$ each with a capacity of 1 . Assume that all bins are initially empty:

Next Fit add the current item, $c_{i}$ (initially $c_{1}$ ), to the current bin $B^{j}$ (initially $B^{1}$ ). Then consider the next item $c_{i+1}$. If it fits in $B^{j}$ then add it. If it does not fit then open the next bin, $B^{i+1}$, and add it. Repeat while there are items left.

Next Fit runs in $\mathcal{O}(n)$ time but provides an approximation factor of $2 \cdot k$, i.e., if $k$ is the optimal number of bins then NF is guaranteed to not use more than $2 \cdot k$ bins.

First Fit add the current item, $c_{i}$ (initially $c_{1}$ ), to the current bin $B^{j}$ (initially $\left.B^{1}\right)$. Then consider the next item $c_{i+1}$. Find the first bin among all the current bins where the item can fit. If there is one such bin then add the item. If there is no such bin then open the next bin, $B^{i+1}$, and add the item. Repeat while there are items left.

\footnotetext{
${ }^{1}$ The decision version of Bin Packing is classified as SR1 in [47, p. 226]
} 


$$
\begin{aligned}
& \text { Listing 7.1: Next Fit Algorithm } \\
& \text { 1: procedure NEXT_FIT } \\
& \text { 2: } \quad k \leftarrow 1 \\
& \text { 3: } \quad B^{k} \leftarrow 1 \\
& \text { 4: } \quad \text { for } i \text { in } 1: n \text { do } \\
& \text { 5: } \quad \text { if } c_{i} \leq B^{k} \text { then } \\
& \text { 6: } \quad B^{k}=B^{k}-c_{i} \\
& \text { 7: } \quad \text { else } \\
& \text { 8: } \quad k=k+1 \\
& \text { 9: } \quad B^{k}=1-c_{i} \\
& \text { 10: } \quad \text { end if } \\
& \text { 11: end for } \\
& \text { 12: end procedure }
\end{aligned}
$$

It is straightforward to have a $\mathcal{O}\left(n^{2}\right)$ First Fit implementation. This can be improved to $\mathcal{O}(n \log n)$ with using appropriate data structures for the search of fitting bin, e.g., heap or priority queues. FF provides a $\frac{17}{10} \cdot k+\frac{7}{10}$ worst case approximation [104].

Best Fit Best fit operates the same way as First Fit. However instead of picking the first bin that has space for the current item during the search phase, Best Fit chooses the tightest fit, i.e., the bin with the smallest capacity left that can still accommodate the current item.

Best Fit has the same worst case complexity and approximation properties as First Fit.

First/Best Fit Decreasing All three algorithms described so far can handle online as well as offline bin packing. However, improvements can be made to these methods for offline packing, i.e., if the items to be packed are all known beforehand. Both First/Best Fit Decreasing algorithms start by sorting the items in non-decreasing order. Then they apply either First/Best Fit to the sorted items.

First/Best Fit Decreasing are some of the best polynomial time approximation algorithm for Bin Packing [59] [58] [99]. These algorithms can have the same time complexities as FF/BF if proper sorting algorithms are applied. However, FFD/BFD have a better worst-case approximation factor of $\frac{11}{9} \cdot k+\frac{6}{9}$ [25].

First Fit Decreasing is the algorithm used as part of some of clustering algorithms implemented in this work. An explanation of the packing problem in the context of task systems for mathematical equation systems and adaptation of the FFD algorithm is presented in Section 7.3. 


\section{Listing 7.2: First Fit Decreasing Algorithm}

1: procedure FFD

2: $\quad k \leftarrow 1$

3: $\quad B^{k} \leftarrow 1$

4: $\quad$ for $i$ in $1: n$ do

5: $\quad$ for $j$ in $1: k$ do

6: $\quad$ if $c_{i} \leq B^{j}$ then

7: break

8: $\quad$ end if

9: $\quad$ end for

10: $\quad B^{j} \leftarrow B^{j}-c_{i}$

11: $\quad$ if $j>k$ then

12: $\quad k \leftarrow j$

13: $\quad$ end if

14: end for

15: end procedure

\subsection{2 k-way Integer Partitioning}

k-way Integer Partitioning, also known as just Partitioning Problem in many Computer Science literature, can be defined as follows: Let $S$ be a multiset of integers :

$$
S=\left\{s_{1}, s_{2}, \ldots, s_{n}\right\}, \quad s_{i} \in \mathbb{N} \quad \forall s_{i} \in S
$$

partition $S$ into a set of $k$ mutually disjoint sub-multisets :

$$
B=\left\{B^{1}, B^{2}, \ldots, B^{k}\right\}, \quad B^{i} \subset S, B^{i} \cap B^{j}=\varnothing \quad \forall B^{i}, B^{j} \in B
$$

so that the sum of elements of each sub-multiset is equal :

$$
\sum_{\forall b_{n} \in B^{i}} b_{n}^{k}=\sum_{\forall b_{n} \in B^{j}} b_{n}^{k} \quad \forall B^{i}, B^{j} \in B
$$

$k$-way Integer Partitioning is NP-Complete. This holds even for 2-way partitioning, i.e., $\mathrm{k}=2$ [47] ${ }^{2}$. A summary of different algorithms for $k$-way petitioning problems can be found in [66].

Makespan Scheduling is a specialized optimization version of $k$-way Integer Partitioning usually presented in terms of Machines and Jobs as follows: p. 223]

${ }^{2}$ The 2-way partitioning problem is classified as SP12 with the name PARTITION in [47, 
Let $S$ be a set of Jobs

$$
S=\left\{s_{1}, s_{2}, \ldots, s_{n}\right\}
$$

Let $M$ be a set of Machines

$$
M=\left\{m_{1}, m_{2}, \ldots, m_{k}\right\}
$$

Let $B_{i}$ be the set of Jobs assigned to Machine $M_{i}$. Furthermore, assume that Job $S_{j}$ takes $t_{i j}$ time units to compute on Machine $M_{i}$. The load $L_{i}$ on Machine $M_{i}$ is defined as

$$
L_{i}=\sum_{\forall s_{j} \in B_{i}} t_{i j}
$$

The maximum load over all machines,

$$
L_{\max }=\max _{m_{i} \in M} L_{i}
$$

is called the Makespan of the job assignment or schedule. Minimum Makespan Schedule is a schedule with the minimum Makespan for a given set of Jobs and Machines.

If all Machines are identical, which means a given job takes the same amount of time units to compute on any machine, then this is an optimization version of $k$-way partitioning. The problem of Minimum Makespan Schedule is NP-hard even for two identical machines.

\subsubsection{Makespan Scheduling Approximation Algorithms}

The problem of finding an optimal Makespan Scheduling for identical processors can be fairly approximated. Similar to bin packing these algorithms can be on-line algorithms that operate without prior knowledge of the jobs to arrive or offline algorithms that require all jobs to be known beforehand.

Greedy List Scheduling This is the simplest approximation algorithm. Greedy List Scheduling considers each item one after the other and assigns it to the processor that currently has the least amount of load. This is quite easy to implement and is shown to have a $2 \cdot k$ approximation guarantee where $k$ is the optimal solution.

Sorted List Scheduling (Longest Processing Time) The greedy List Scheduling can be improved for off-line scheduling, i.e., it is possible to analyse or pre-process the jobs before starting scheduling. One such algorithm is the Sorted List Scheduling. It is a simple algorithm that sorts the jobs in non-decreasing order before applying the greedy List Scheduling. This preprocessing improves the approximation factor to $\frac{4}{3} \cdot k$ [50]. 


\section{Figure 7.1: Merge Single Parent}

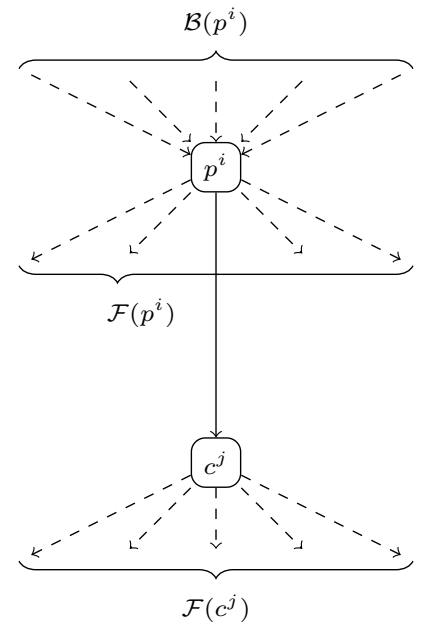

(a)

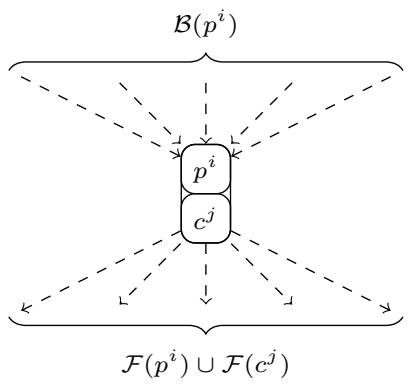

(b)

\subsection{Clustering Heuristics}

Multiple clustering heuristics have been investigated and implemented in this work. Some of these heuristics are intended for reducing the complexity and improving locality while others also try to perform some variant of load balancing. Some of these heuristics build up on the classic optimization problems mentioned above. All the currently available heuristics are discussed in the sections that follow.

\subsubsection{Merge Single Parent (MSP)}

The simplest clustering available is the merge single parent (MSP) rule demonstrated in Figure 7.1. This clustering algorithm traverses the system graph and merges all tasks that have only one parent task to their parents.

There are two main advantages with this simple rule. The first advantage is that it eliminates the need for treating cheap tasks, i.e., tasks with low execution time, separately by the schedulers thereby reducing overheads for schedulers without losing any potential for parallelism. Furthermore, since a dependency (parenthood/childhood) means sharing of some data between the two tasks, it improves temporal locality (cache for CPUs and local/shared memory spaces for GPUs) and makes sure data is used as soon as it is available for the next task. If a cheap child task is left unmerged then it might end up further away from the parent task in terms of execution order either 


\section{Figure 7.3: Merge Level Parents}

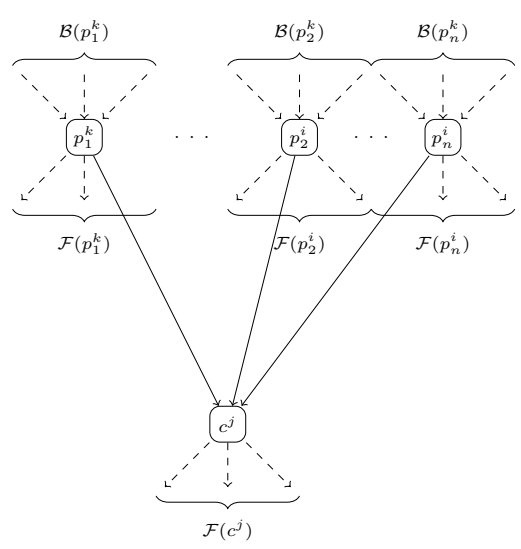

(a)

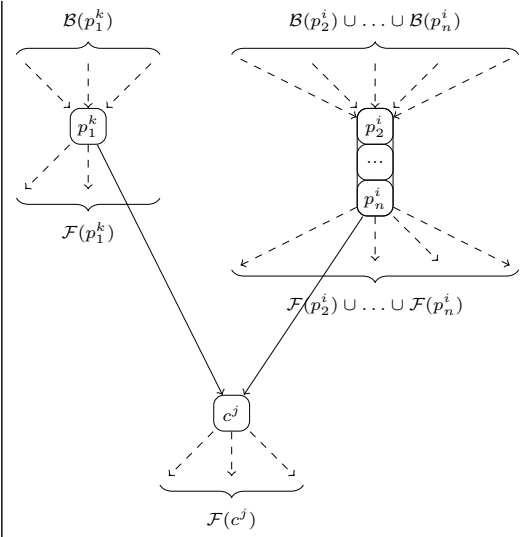

(b)

due to application of other clustering rules or scheduler task selections. This will result in a poor temporal locality for data without any gain in potential parallelism.

\subsubsection{Merge Level Parents (MLP)}

The second available algorithm is called Merge Level Parents (MLP). This clustering rule utilizes the level of a node and parenthood relationships between nodes to apply merging rules. It starts by finding the level of each node in the task graph. Then nodes are traversed in a breadth first manner starting from the third level (level 0 contains only the root node and nodes at level 1 have no parent apart from the root node). Then the merging rule is applied as shown in Figure 7.3.

The rule visits each non root node or task and merges its parents. If the node has only one parent then nothing is done. Otherwise parents of the node are merged depending on level and cost criteria. The parents of the node are collected based on their level, i.e., parents of the same level are put in the same group. Next it will go through each group and merge the parent nodes depending on the cost criteria. If cost is not relevant, signified by having a cut-off cost of zero, then all parents of the same level are merged. If however we have a specific cut-off cost then the grouped parents are merged with consideration of cost. This is achieved by the Merge Level for Cost heuristic that is discussed in Section 7.3.4. The motivation for having a cut-off cost is simple. Merging nodes in this form reduces the available potential parallelism. Therefore, sometimes it can be beneficial to not merge big-enough tasks even 
Listing 7.3: MLP clustering

1: procedure MLP_BFS

2: $\quad c_{0} \leftarrow$ cutoff_cost;

3: $\quad$ for $u \in$ BFS_VISIT() do

4: $\quad$ if $|\mathcal{B}(u)|>1$ then

5 :

$6:$

$L[m][n]=$ COLLECT_SAME_LEVEL $(\mathcal{B}(u))$;

for $i$ in $0: m$ do

if $c_{0}==0$ then

MERGE_ALL $(L[i])$;

else

end if

end for

end if

13: end for

15: end procedure

if it reduces the amount of tasks we have to deal with in the end. The pseudocode for MLP heuristic is shown in Listing 7.3.

Parents are not merged if they are different level tasks. This restriction is enforced in order to avoid creating paths that will potentially lead to cycles in the graph. For example consider the system shown in Figure 7.5. If the MLP rule is applied to node 5 of this task system without the level restriction on parent node merging the system will end up with a cycle between the merged nodes 1,3,4 and 2. Although it is possible to check if merging of nodes would introduce a cycle prior to performing a merge, we have opted not to do this for performance reasons. Path finding is an expensive operation and performing it for every single merge can severely impact the efficiency of the whole algorithm.

\subsubsection{Merge Level for Bins (MLB)}

The number of processing units used to evaluate a given task system is usually known beforehand and is fixed. For example if the parallelization is to be performed on a typical machine such as the desktops and laptops that we have, the number of cores is known beforehand. Certain schedulers can benefit from this information. For example for the level scheduler available in this implementation the number of processing units can be used to further reduce the number tasks in the final task graph. Since a machine with $m$ computing units can only evaluate at most $m$ tasks simultaneously we can further merge the graph to have only $m$ tasks per level. 


\section{Figure 7.5: Cycles in parent merging}
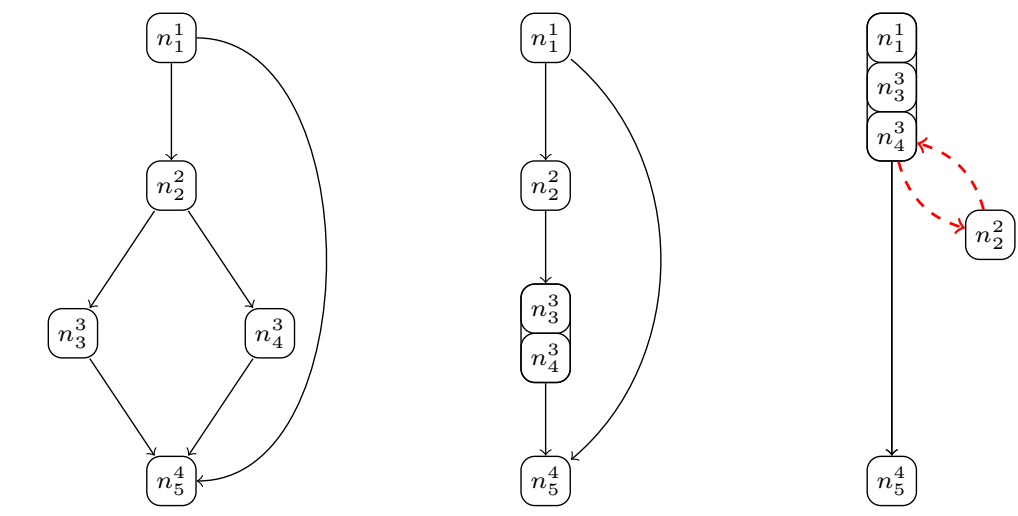

However, this $m$ tasks should have costs that are balanced, otherwise the evaluation can be dominated by the largest task increasing the effective cost. The Merge Level for Cost rule performs this clustering and tries to generate a balanced system of $m$ tasks from each level of the input task system.

The clustering starts by computing the levels of each node in the task graph. Then it traverses the graph collecting all nodes of the same level into the same group. Since each node in the same level group is guaranteed to be independent of each other we can further partition it into $m$ merged tasks without violating any dependencies. That is, given $m$ machines and a set of $n$ tasks with costs

$$
\mathbf{C}=\left\{c_{1}, c_{2} \ldots, c_{n}\right\}
$$

we would like to partition $C$ into $m$ mutually disjoint subsets of costs

$$
\mathbf{C}^{M}=\left\{C_{1}, C_{2} \ldots, C_{m}\right\}
$$

such that

$$
C_{\max }=\max _{i \in m} C_{i}
$$

is minimized. This is a make-span scheduling problem discussed in Section 7.2.2. Since all tasks and their costs are known beforehand we can apply the Longest Processing Time algorithm to achieve the clustering. Each group of the collected same level tasks is merged into a balanced set of a maximum of $m$ tasks.

Each level of the collected same level tasks is sorted by cost. Then each level or group is traversed as follows. The first $m$ tasks are picked as merged tasks. The next available task, $\operatorname{tas}_{m+1}$, is merged with the smallest cost merged task. This process is repeated until there are no tasks left. The pseudo-code for MLB heuristic is shown in Listing 7.4. 


\section{Listing 7.4: Merge Level for Bins}

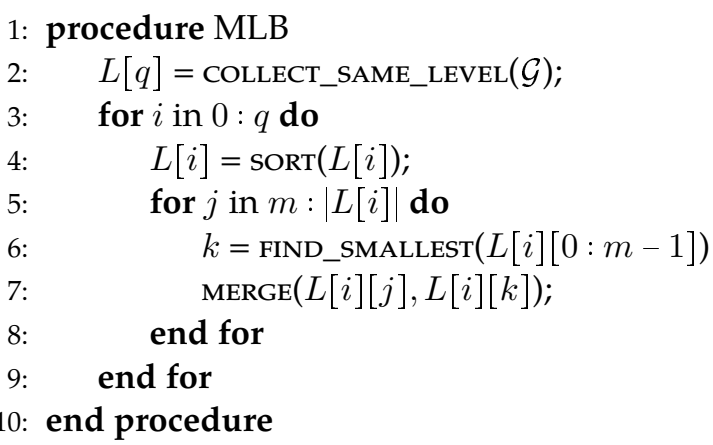

\subsubsection{Merge Level for Cost (MLC)}

If the number of processing units is pre-determined then the MLb heuristic presented above is usually what is needed. However, in some cases it might be beneficial to have more tasks than the processing units. This can allow, for example, to have a scalable task graph which can be used in a machine with more processing units. It might also be beneficial to have more tasks to lead to a possibly better runtime balancing. The higher number of tasks can for example be computed in a dynamic parallel loop. This means even if some tasks have increased costs after the original scheduling it might be possible to still have a balanced execution by dynamically assigning the task to the currently free processing unit.

However, we still have to make sure that we do not result in a graph with too many small tasks where the overhead of processing or dynamically assigning tasks is higher than the benefit of potential parallelism. This can be enforced to some extent by selecting a cut-off cost where we think that a task is worth keeping by itself. The heuristic starts by computing the levels of each node in task graph similar to MLB. However, in this case the target is to generate as many as possible merged tasks while making sure that there no task is left when it can be merged without violating the cut-off cost limit. That is, given a set of $n$ tasks with costs

$$
\mathbf{C}=\left\{c_{1}, c_{2} \ldots, c_{n}\right\}
$$

we would like to partition $C$ into a number mutually disjoint subsets

$$
\mathbf{B}=\left\{B_{1}, B_{2} \ldots, B_{m}\right\}
$$

such that

$$
\sum_{x \in B_{i}} x \leq \boldsymbol{c c}, \quad \forall B_{i} \in \boldsymbol{B}
$$

where $c c$ is the cut-off cost. 


\section{Listing 7.5: Merge Level for Cost}

1: procedure MLC

2: $\quad L[q]=$ COLLECT_SAME_LEVEL $(\mathcal{G})$;

$4:$

5

6:

7:

8:

9:

10:

11:

12:

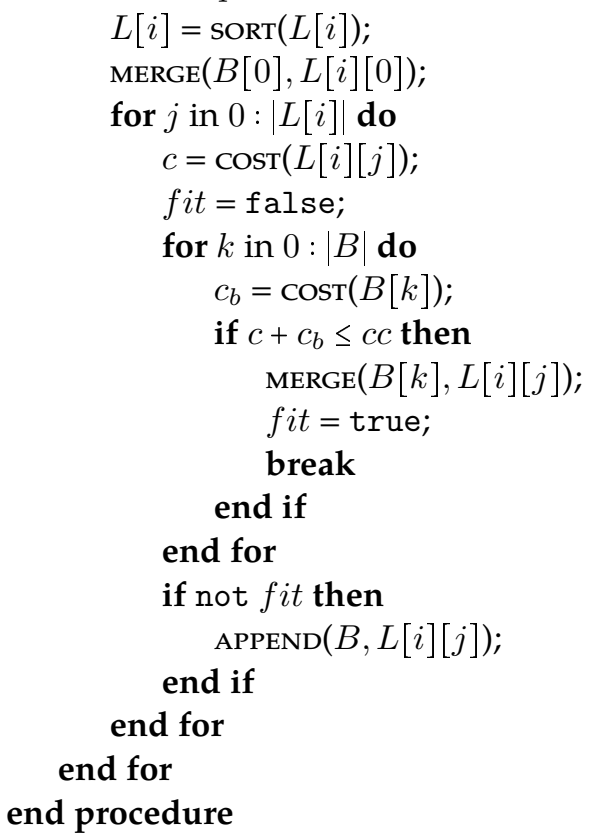

This is the bin-packing problem discussed in Section 7.2.1. Since all costs are known at scheduling time we can apply an off-line approximation algorithm in this case. The current implementation applies the First Fit Decreasing algorithm. Each level of the collected same level tasks is sorted by cost. Then each level or group is traversed as follows. The first tasks, that is the largest task, is picked as the first bin. Then the heuristic traverses each remaining tasks and searches for a task that can fit in the first bin. If such a task is found then it is merged with the current bin and searching starts again. If there is no task that can fit the current bin then the first task in the remaining un-merged tasks is picked as the new bin and the whole process is repeated. The pseudo-code for MLC heuristic is shown in Listing 7.5.

The selection of the cut-off cost should be considered carefully. Since it provides the trade-off between complexity and potential parallelism, it can have significant consequences in the overall performance of the parallelization. Selecting a too high cut-off cost can for example, in the worst case, result in a single task on each level of the graph, while a too small cut-off cost can leave around many small tasks that are not worth processing individu- 
Figure 7.6: FourBitBinaryAdder model equation structure after symbolic processing

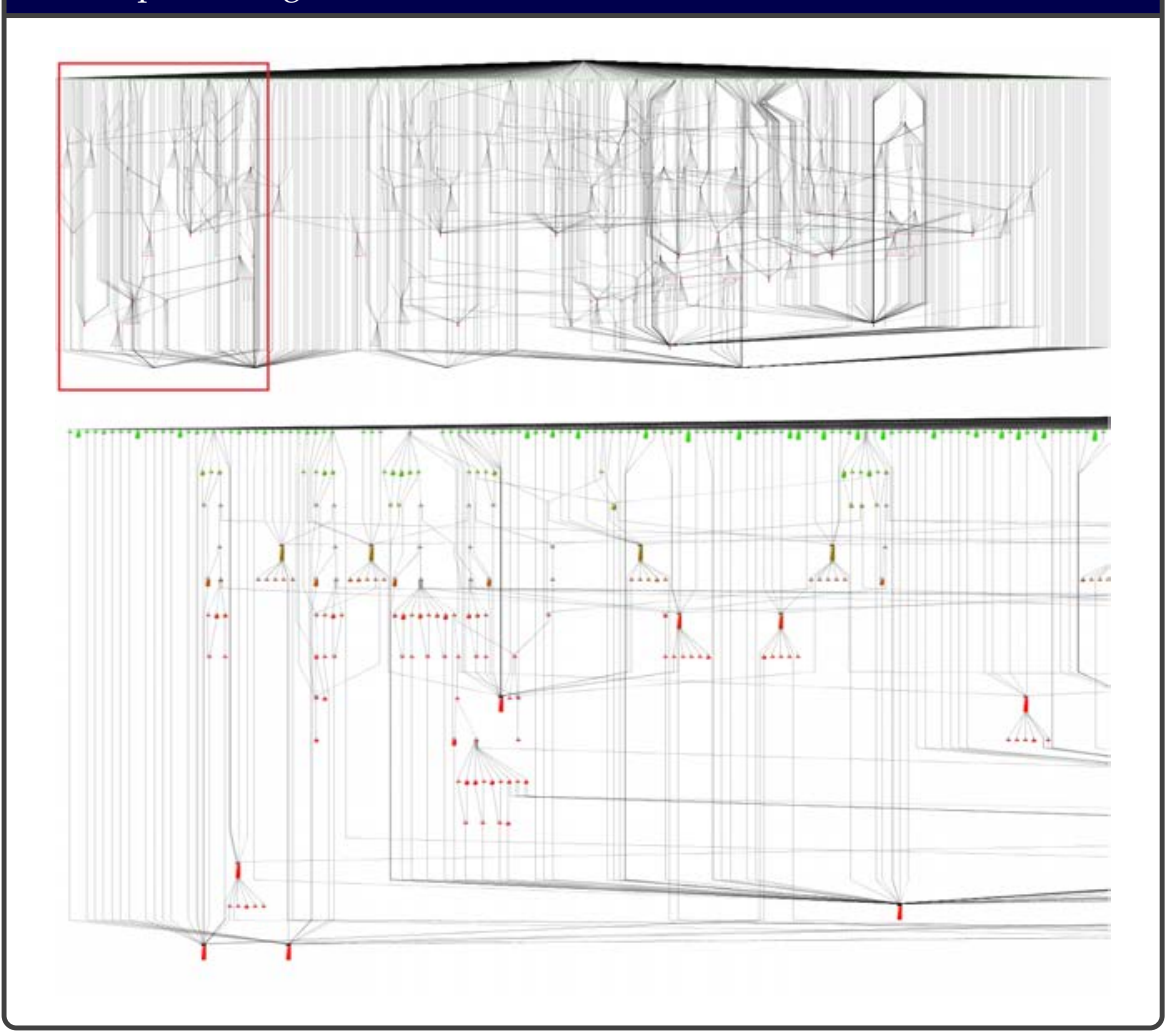

ally. However, obviously, there is no universally convenient cost to be used, since differences in architecture and intended use govern the decisions to be made. For example, a task can be considered too small on a fast architecture with a high thread context switching overhead. On the other hand it might be better to keep it separate on a slow architecture but with very efficient thread management such as modern GPU architectures.

\subsection{Schedulers}

The scheduler concept in this work is mostly concerned with the synchronizations and management of tasks. This might be a bit different from the usual view of schedulers that are responsible, to some extent, in specifically "assigning" a serious of tasks to a specific processing unit. That means the schedulers are dependent on the clustering heuristic discussed in the previous section for reducing the complexity of the system or generating balanced assignments wherever needed. Both schedulers can accept any of the cluster- 


\section{Figure 7.7: FourBitBinaryAdder model equation after applying MSP, MLP and MLB}
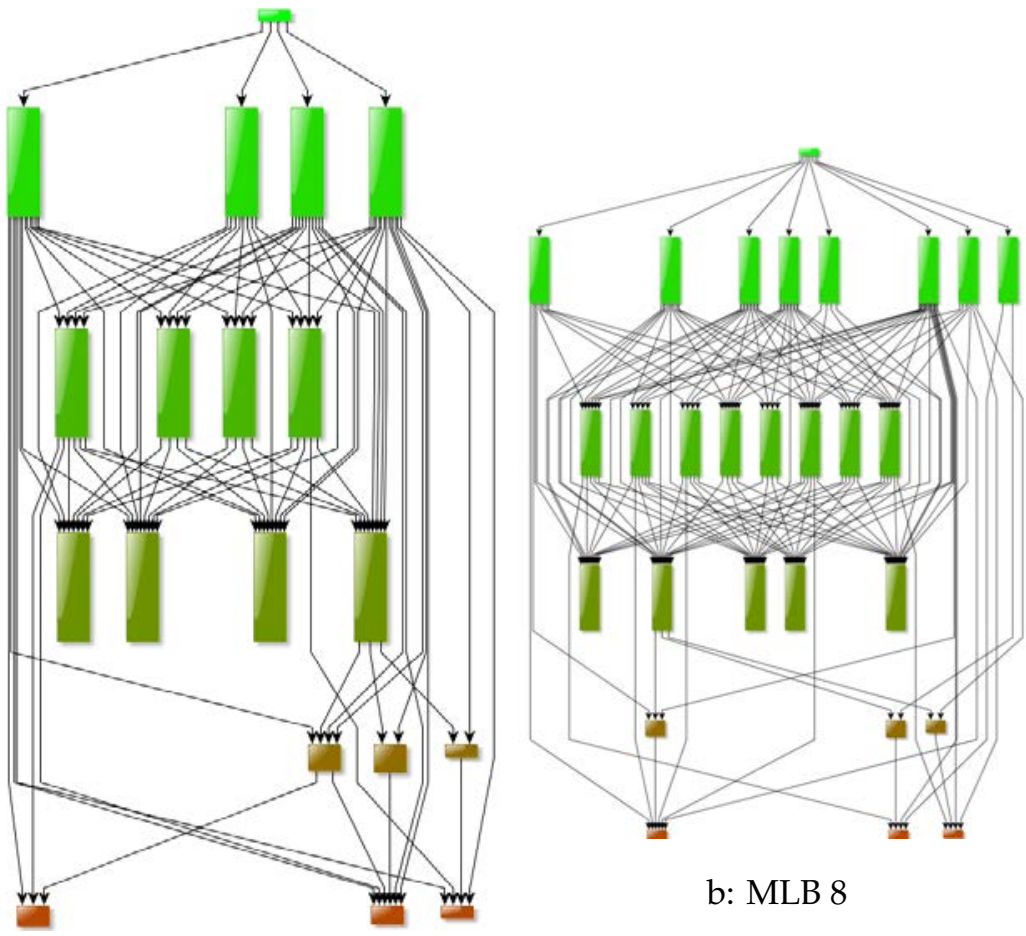

b: MLB 8

a: MLB 4

ing heuristic as pre-processing stages and apply them in order whenever a new schedule is requested. This allows for a flexible approach where a given scheduling approach, e.g. a level scheduler, can be easily configured for systems of different structure.

There are two scheduler implementations available at the moment. The first is the so called Level Scheduler or Wave-front Scheduler.

The second available scheduler is the so called Flow Graph Scheduler. This scheduler is designed as a wrapper to the Intel TBB [tbb] Flow Graph (TBBFL) implementation. Intel TBB Flow Graph implements its own a message based work stealing algorithm to dynamically execute tasks in the graph.

\subsubsection{The Level Scheduler}

This is an adaptive semi-static scheduler that is based on a step and synchronize mechanism. The scheduler starts by traversing the input graph and comput- 
ing the level of each node in the task graph. Then it collects all nodes of the same level together. Since all nodes of the same level are guaranteed to have no dependence on each other they can be directly executed in parallel. The scheduler launches each level of the graph in parallel and synchronizes in between successive levels.

Clustering This is a simple scheduler with very little management overhead. Due to this it is very appealing in most cases. However, if this scheduler is to be efficient, then each level of the graph should result in a balanced parallel execution. This balancing can be achieved by providing specific clustering heuristic to the scheduler. The clustering heuristic associated with the scheduler are applied to the task graph by the scheduler prior to execution.

Profiling Generating a balanced execution of a level requires knowledge of the costs of each task in the original task graph. The scheduler is responsible for profiling the input task graph to collect this cost information if requested. If the input graph has no information then the scheduler will always execute the task graph sequentially in topological order while profiling the cost of each individual task. Once the cost information is available the scheduler applies the requested clustering rules and generates the final graph. This resulting task graph is then executed in parallel for any subsequent requests for evaluation.

Rescheduling The scheduler is adaptive. That is, at any point during the successive evaluations, a rescheduling can be performed. This rescheduling can be explicit requested by the runtime system using the scheduler or they can be governed by specific rescheduling policies internally. In order to provide the rescheduling capability the scheduler internally maintains a copy of the structure of the original task graph. If a rescheduling is to be done at some point the scheduler switches to this original graph and performs a sequential profiling step recording new cost informations for each task in the system. This new cost information is then used to reapply the associated clustering rules to the graph resulting in a new task graph that will be used for the subsequent evaluations.

At the moment there are two possible internal rescheduling rules. The first one is a simple periodic rescheduling where a new schedule is generated every $n^{t h}$ evaluation. This rescheduling approach is capable of handling different execution behaviours. It can, however, be expensive if the period is too small since profiling sequential steps take considerably more execution time relative to normal evaluations. If the period is too large then it might be too slow in responding to changes. The second rescheduling policy used is a running average based rule. This policy is based on computing the average execution time of all previous parallel evaluations since the last scheduling 


\section{Figure 7.8: Level Scheduler operation flow.}

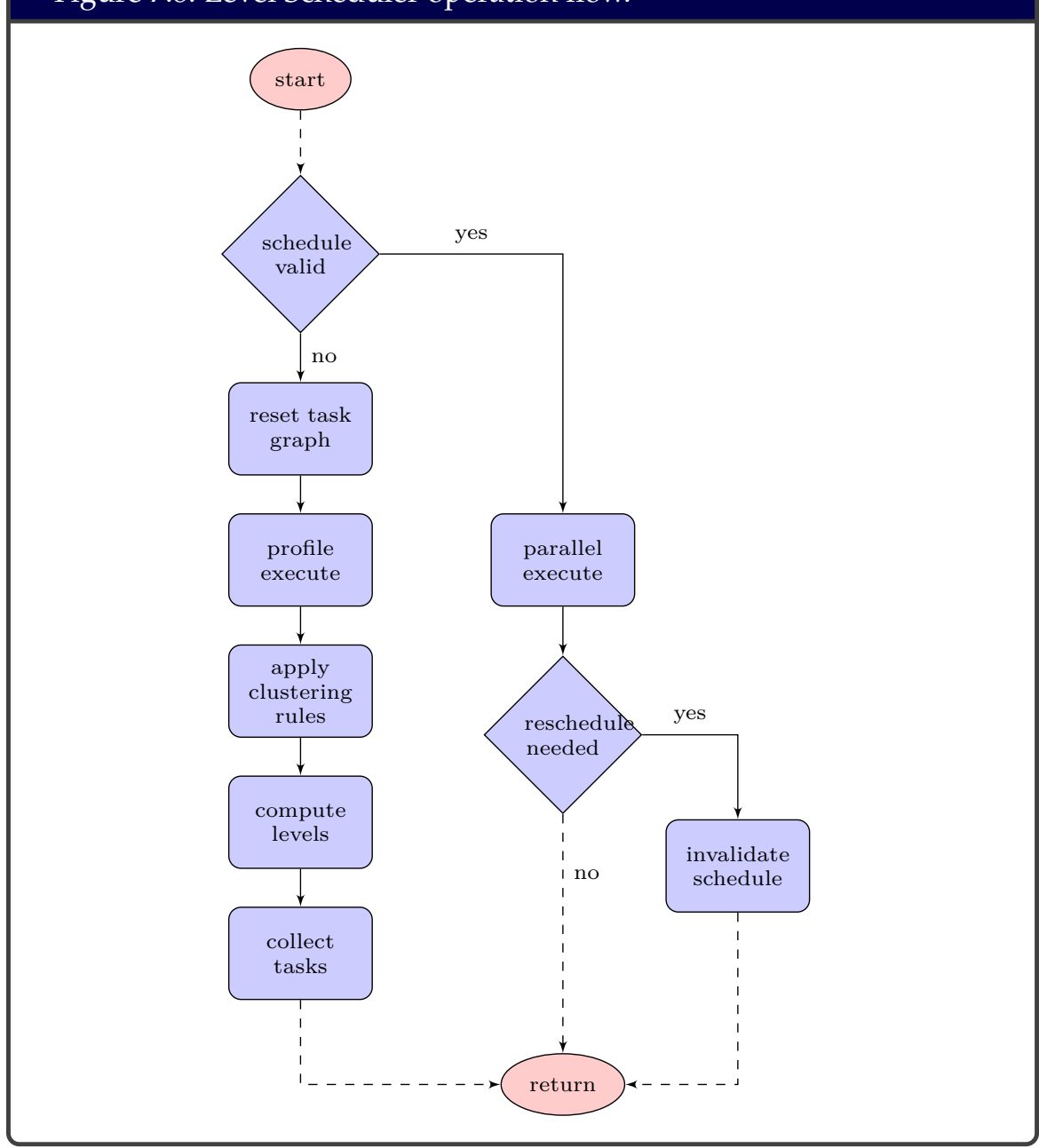

point. Whenever a new scheduling is performed the execution time of the last parallel evaluation before the rescheduling is recorded as a reference point. If at any point the current running average diverges too much above or below the recorded value a new rescheduling is performed and the last parallel evaluation cost is kept as a reference.

Level Evaluation The maximum number of threads the scheduler can use to perform parallel execution at any time can be adjusted. The clustering rules provided to the scheduler may result in a task graph that can potentially have more tasks at any level the maximum number of threads allowed. Therefore, the scheduler should be able to further decide each task should be executed. 
This is done in a simple round-robin manner. In the general case it is a good choice to provide a balancing clustering rule such as MLB heuristic discussed earlier as the last clustering rule for such scheduler if it is possible to generate a satisfactorily balanced structure.

Figure 7.8 roughly summarizes the complete operation flow of the Level Scheduler on each evaluation of the task graph.

\subsubsection{Flow Graph Scheduler}

This scheduler is a wrapper around Intel TBB's flow graph scheduler. This is a message passing dynamic scheduler based on a reference counting mechanism. At the time of creation each node keeps count of the number of predecessors it has. Up one completion of its execution a node tries to queue all its successors. The successor nodes increment their count internally for every queueing attempt. The nodes start executing when the count of queuing attempt is equal or more than their predecessor count. After the end of execution the counter is reset and the task attempts to enqueue all of its successors.

The reference counting mechanism adds some overhead to the execution of the graph. Directly applying this scheduler to large task systems can result in a too much overhead. For example most of the small tasks in a simulation system are made up single assignment statements. Reference counting for every single assignment task can end up in more time spent on management than it actually takes to execute the task. For this reason the creation of the flow graph can be preceded by a clustering phase. Similar to the Level Scheduler a set of clustering policies can be associated with each Flow Graph scheduler specialization. This rules are applied before the first execution of the task graph.

In addition to providing an extra scheduler option, this scheduler serves as point to compare and contrast the performance and usability of a semistatic scheduler such as the level scheduler discussed above versus a completely dynamic scheduler. The benefit of each scheduler dependent on the structure and runtime behaviour of the task system at hand. An overview of the comparisons in performance between the two schedulers can be seen in the test case results presented in Chapter 9. 


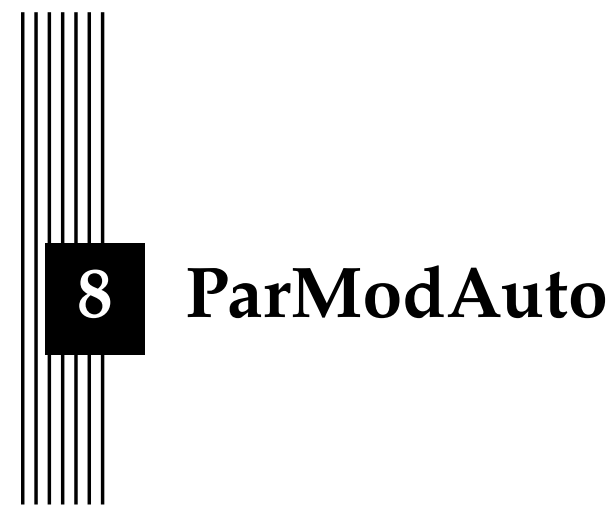

\subsection{Motivation}

There have been multiple parallelization research efforts and implementations related to Modelica and the mathematical modeling and simulation area in general. Almost all of these approaches and implementations are focused on automatic parallelization where the simulation tool or compiler is responsible for extracting and utilizing parallelism. It is not surprising to find that many of these implementations share a lot of ideas even though they were designed for different tools by different researchers. For example many of these approaches, see [7] [73] [102] [28] [19], use a graph based representation for dependency analysis with very similar task merging or clustering heuristics operating on this graph to construct balanced parallel executions.

One of the problems with all these implementations is that they are all tied to a specific tool and environment. Despite the fact that most of them use similar approaches and methods there is no portable way of moving one implementation to another to improve or extend functionality. All implementations have to start from scratch and go through the mundane and error prone processes of extending already complicated environments with even more complicated parallelization related processing. The ParModAuto task system parallelization library tries to address this complexity, extensibility and portability issues by providing a stand-alone task system parallelization implementation that is easy to integrate into existing systems. 


\subsection{Design Principles}

We have tried to follow some design principles to ensure that the ParModAuto library is flexible and efficient. First of all it should relieve researchers from reinventing the wheel every time they want to try out a new algorithm or heuristic that can utilize or improve parallelism. In addition to simplifying the technical effort needed to investigate a parallelization, we have provided implementations of the common heuristics used by many of the previous approaches as well as some more advanced clustering and scheduling approaches discussed in Chapter 7. The subsequent subsections will summarize the main principles which governed the design of the ParModAuto Task System Parallelization library.

\subsubsection{High Level Operation}

ParModAuto is a high level parallelization library. It is designed to encapsulate and hide the low-level parallelization details. Half of the difficulty of a parallel programming effort typically lies not in the design and implementation of features but rather in handling mundane technical problems and errors. The idea of ParModAuto is that the users' focus should be on their own algorithms or heuristics. The library provides a high level representation of tasks, clusters and schedulers to simplify this process.

Users do not need to interact with the underlying low-level framework or API such as OpenMP [12] or PThreads [13]. There is no need to interact directly with any thread creation and management from the end-users' perspective. Execution control is available at the whole system level only. Under normal operation the whole system is executed or nothing is executed at all. However, end-users have control over which clustering algorithms should be applied, what scheduler is used, and can also set certain parameters such as cut-off costs for task merging. Of course, when extending the library with new scheduling algorithms it is possible to have direct low-level control over all these things.

ParModAuto is implemented on top of the Boost Graph Library (BGL) [16]

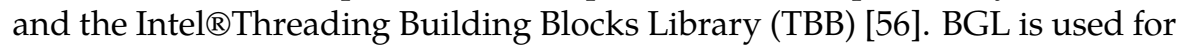
the underlying graph primitives and TBB for the multi-threading functionalities.

\subsubsection{Runtime Processing}

One of the most important factors for effective clustering/merging and scheduling of tasks is cost estimation. Before any scheduling is done, it is necessary to have a good estimate on the cost of each task. This cost estimation can be done in two ways: Static or Dynamic cost estimation. Static estimation is where cost is assigned from a predetermined set of estimations for 
each type of task. In Modelica based models the compiler can do this estimation at compile time by analysing the expressions that make up an equation. On the other hand dynamic cost estimation is based on profiling information. Tasks are executed at least once and execution times recorded. This information can then be used to generate an execution schedule.

Static cost estimation has been used in many parallelization efforts in Modelica environments. [7] [73] [28] estimate the cost of evaluating each equation at compile time with the help of a compiler. [102] uses an semi-static approach where profiling information from a previously completed simulation is used for subsequent simulations.

ParModAuto is based on runtime analysis. Profiling and cost information is recorded and used during an active simulation. In contrast to other implementations, graph creation, analysis, clustering or task merging, and scheduling are all done at runtime. This allows for an adaptive balanced execution over the available computational resources.

It is possible to address system load or even architecture variations between multiple runs since each simulation is self contained in terms of information needed for parallelization. Profiling and rescheduling can also be done multiple times during a single simulation. This means that it is possible to respond to variation not just in system load but also in the actual behaviour of the system being simulated due to changes such as numeric differences or event triggers.

\subsubsection{Portability Considerations}

The implementation should be portable. Portability in this context is relevant in two ways.

Tool Portability It should be possible to use the library on different tools with minor interfacing effort. For this reason we have very few assumptions on what a tool will provide. Representations should be generic and not concerned with tool specific compile time or runtime implementations. For example the library makes no assumptions about what solver is used, how linear or non-linear systems are handled, how events are handled and so on. A modified version of the implementation that uses the Boehm Garbage Collector [14] [15] is also available to accommodate tools with garbage collected runtime systems.

Architecture Portability As mentioned earlier the design is aimed at hiding the details of low level issues such as thread creation and management. This means that users will not have to deal with portability issues arising from using a certain parallelization API or framework directly, e.g., OpenMP or POSIX Threads. For example the connected component parallelization discussed earlier by generating the OpenMP constructs directly into the rest of 
the simulation code. Most of the effort was spent in making sure that changes needed to support parallelization did not affect normal operation, e.g. generated code should be compilable in platforms that do not support OpenMP. Experiences gained from previous attempts has led us to add one layer of encapsulation by using the Intel ${ }^{\circ}$ Threading Building Blocks library [56] to improve portability and scalability.

Performance Portability In addition to being platform portable the operations performed should be performance portable. The gained performance improvements should not be tied to a specific architecture. This is achieved to some extent by the use of Intel's TBB library which is expected to be well tested and adjusted to perform on wide range of architectures.

\subsubsection{Extensibility Considerations}

Users should be able to easily extend ParModAuto with their own algorithms or heuristics. The library should readily provide the information needed to design new clustering/merging algorithms or implement schedulers. For example cost data and dependency information, i.e., parents and children for each task, and basic traversal routines are readily available. In addition the library provides some basic task merging routines, that can take cost into consideration if needed, to ease up construction of more complicated heuristics or algorithms.

\subsubsection{Independence: Minimal Assumptions}

The design is intended to be as independent as possible. Even if the library is designed with the OpenModelica simulation environment and compiler in mind, experience with previous implementation has shown a closely tied approach will lead to unnecessary complications in the tools that use the library. The current design approach tries to make the minimum assumptions about what a tool or a compiler will provide with regards to parallelization. The whole aim to make the compiler as oblivious as possible to the parallelization process. One example of choices made due to this preference is the construction of the Directed Acyclic Graph used for dependency information at runtime instead of at compile time as explained in Section 8.3.3. decisions such as this are made even in the face incurring extra overhead in computational time in order to minimize the amount of change needed to be done to the compilers or tools that intend to utilize the parallelization implementation.

The design is also intended to be impervious to other simulation related choices such as solver selection. It makes no assumptions in what specific solvers provide or how they operate. It is solely concerned with executing a set of tasks with their specified dependencies. This means that a parallelized 
simulation can select its solver freely even after compilation if the tool allows it. It is also intended not to interfere with other simulation specifics operations such as event handling, logging and so on.

\subsection{Implementation}

\subsubsection{Task Abstraction}

A user defined task (think equation in this context) is represented as a $\mathrm{C}++$ class. This class must inherit from the abstract class TaskNode which provides the interface expected by the clustering and scheduling classes. A simplified task representation of a Modelica equation (simple or complex) is shown in Listing 8.1. An instance of this class is created for each equation that is part of original system.

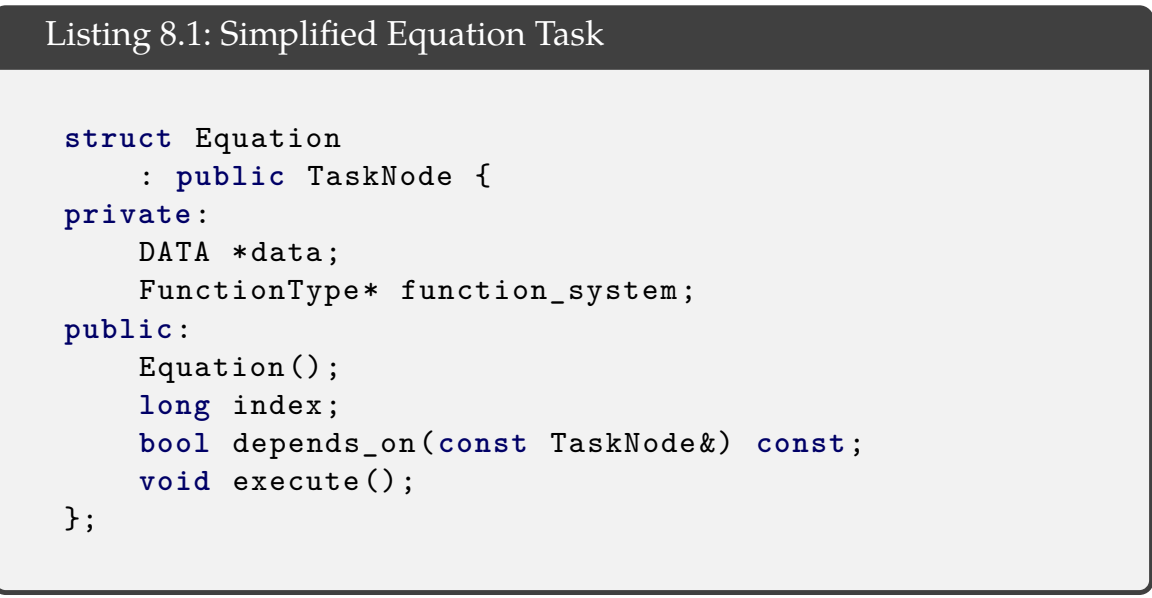

\section{Listing 8.2: execute Function definition for Equation functions}

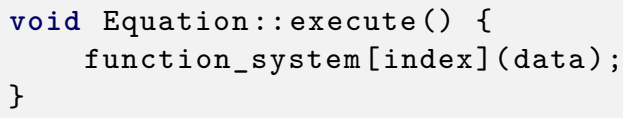

This external representation of an Equation by ParModAuto means that the code generated by the compiler or tool needs very minor modifications. For example consider the actual execute() method of the prototype OpenModelica implementation is shown in Listing 8.2. The only modification needed to allow this execution from the OpenModelica code generators side 
is to generate an array of function pointers to the functions representing each equation.

\subsubsection{Clusters}

Even though we refer to the complete representation of a mathematical equation system by the ParModAuto library as TaskSystem, the actual nodes in the graph are Clusters.

\section{Listing 8.3: A ParModAuto Cluster Class}

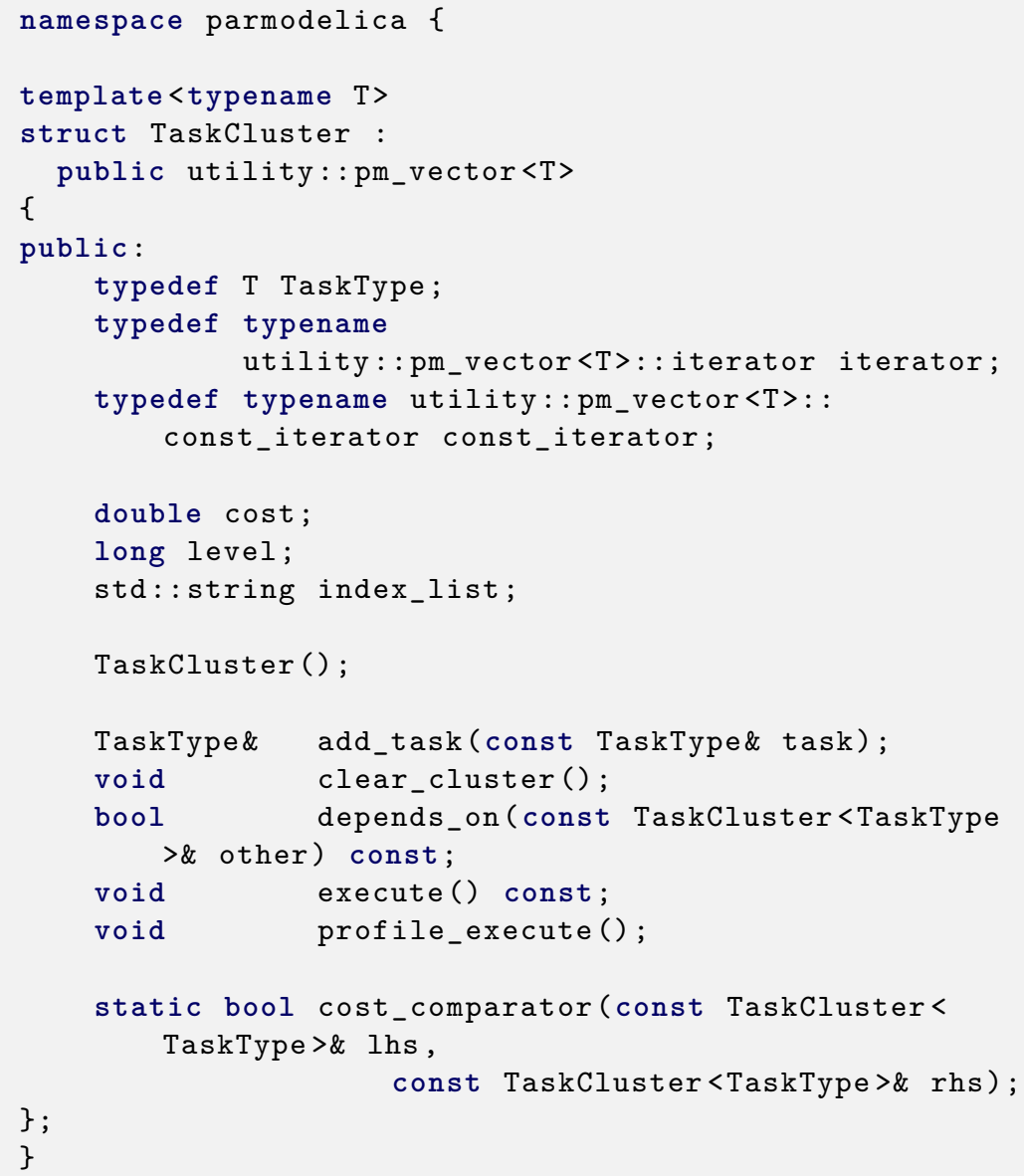

A cluster is just a collection of tasks. When a node - a node representing an equation in the model - is added at the initial creation of the graph a new 
cluster is created and added to the graph. his cluster initially contains that specific one task or equation. After the first profiling execution step is done the different clustering algorithms rewrite the system graph by moving tasks from one cluster to another. Each clustering algorithm decides where and how to move tasks from cluster to cluster. These operations are discussed in detail in Chapter 7.

All tasks in the same cluster are always executed sequentially in the order they are added to the cluster. If and when a cluster becomes empty due to one of these task movements it gets detached from the system and is destroyed. A simplified bare-bone cluster class from the current implementation is shown in Listing 8.3. The cluster class is a template class which means it can work on any task abstraction that satisfies the TaskNode class requirements. Clusters can also analyse dependency by going through all the tasks in the cluster. This is useful if there is a need to restructure the graph after applying any of the clustering algorithms. During normal operation, when tasks are moved from cluster to cluster, all dependency information is updated automatically, i.e., edges are created and destroyed accordingly.

Clusters are also responsible for the profiling of their tasks. They have two modes of execution: normal and profiling executions. In normal execution mode tasks in the clusters are simply executed in the order they are added to the cluster. In profiling mode, however, the cluster records execution time for each task one by one and updates the task nodes with the resulting cost information. This cost information is then used to perform clustering or scheduling operations.

\subsubsection{Dependency Specification and Task System Construction}

Dependency information is needed in order to construct a task graph that can be manipulated and executed accurately. In the context of Modelica models, the compilers can be reasonably expected to have this information at code generation time since most of the processing in a mathematical processing back-end of a Modelica tool is heavily reliant on dependencies between equations. However, transferring this information from the compiler to the ParModAuto library to be used at runtime is a matter of interest.

\section{Listing 8.4: Dependency JSON file}

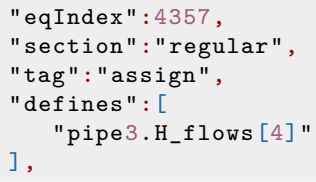


Listing - Continued

"uses" :

"pipe3.mediums [4].h",

"pipe3.mediums [3].h",

"pipe3.flowModel.m_flows [4]"

]

"equation" : [

"semilinear (pipe3.flowModel.m_flows [4], pipe3.mediums [3].h, pipe 3 .mediums [4].h)"

],

"source" : \{

"info": \{

"file": "D:/OpenModelica/build/lib/omlibrary/Modelica 3.2.1/

Fluid/Pipes.mo",

"I ineStart" : 494 ,

"IineEnd" : 494 ,

"colstart" : 9 ,

"colEnd" : 76

\},

"within" :

"Modelica.Fluid.Pipes. DynamicPipe \\$pipe3.\\$for loop scope $\backslash \$ . \backslash$ \$for loop scope $\ \$ "$ 


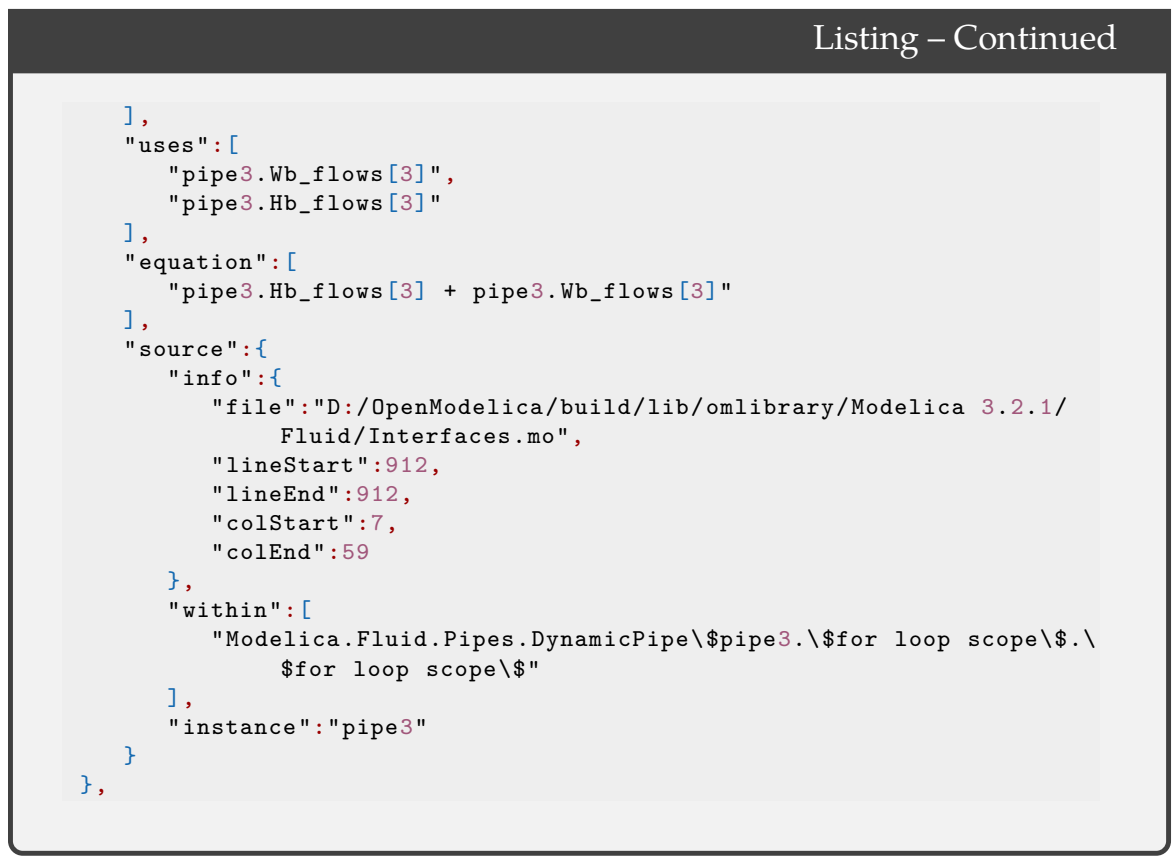

ParModAuto provides the basic methods for directly creating graphs as well as nodes and edges on this graph to create a representation of the equations and dependencies between them. Currently the analysis and creation of the dependency graph is done at runtime. This means the cost information for every single task or equation evaluation can be estimated much more accurately from runtime profiling information as compared to compile time cost estimation based on preset cost values assigned to each expression. It is technically possible to create the task graph with the complete dependency information at compile-time and augment this graph with profiled cost information at runtime. In fact this approach might save a bit of execution overhead since the graph will not be reconstructed live at runtime every time the model executable generated by the compiler is executed, i.e., simulated. In other words we can compile once and simulate multiple times without recreating the graph on every run. However, this approach is avoided for multiple reasons. First of all the relative time spent on creating the task graph at runtime is negligible for most fairly complex models. The actual simulation operation - evaluating the equations of the system - thousands of times during each simulation greatly outweighs and shadows the cost involved in creating the task graph. Secondly the implementation complexity of keeping track and augmenting each task node created at compile-time and then augmenting it at runtime can in itself incur another overhead. In addition a compile-time graph creation approach means that any compiler that wants to use the ParModAuto implementation will require considerable modifications. 
This modification can further complicate the already complicated process of handling complex mathematical models in the compiler back-ends. One of the main design consideration in the implementation is that it should require only the absolute minimum modifications from the compiler or tool side.

The current prototype implementation in the OpenModelica compiler uses the JSON ${ }^{1}$ file format to dump the dependency information. The file format specifies which variables are updated and used by each SCC or block of equation. An example dependency json file for the Modelica.Fluid.Examples. BranchingDynamicPipes model from the Modelica Standard Library (MSL) is shown in Listing 8.4. ParModAuto constructs a task graph by analysing the variable usages in each equation and deducing dependencies accordingly. In this example equation 4358 uses variable pipe3.H_flows [4] which is defined (assigned to) in equation 4357 resulting in an edge $4357 \rightarrow 4358$ in the task graph.

\subsubsection{Task System Representation}

The next level of abstraction after clusters is the actual graph itself. The TaskSystem class of the ParModAuto library provides this representation. This class uses the Boost Graph Library's (BGL) adjacency list representation internally and provides a wrapper class with additional functionalities. This is also a template class with expecting a TaskNode derived class (See Section 8.3.1) type as a template parameter. A simplified listing of the TaskSystem Class presenting the interface is shown in Listing 8.5. This class is responsible for constructing the Directed Acyclic Graph representation of the equation system from the dependency specification generated from the compiler or tool such as the one shown in Listing 8.4.

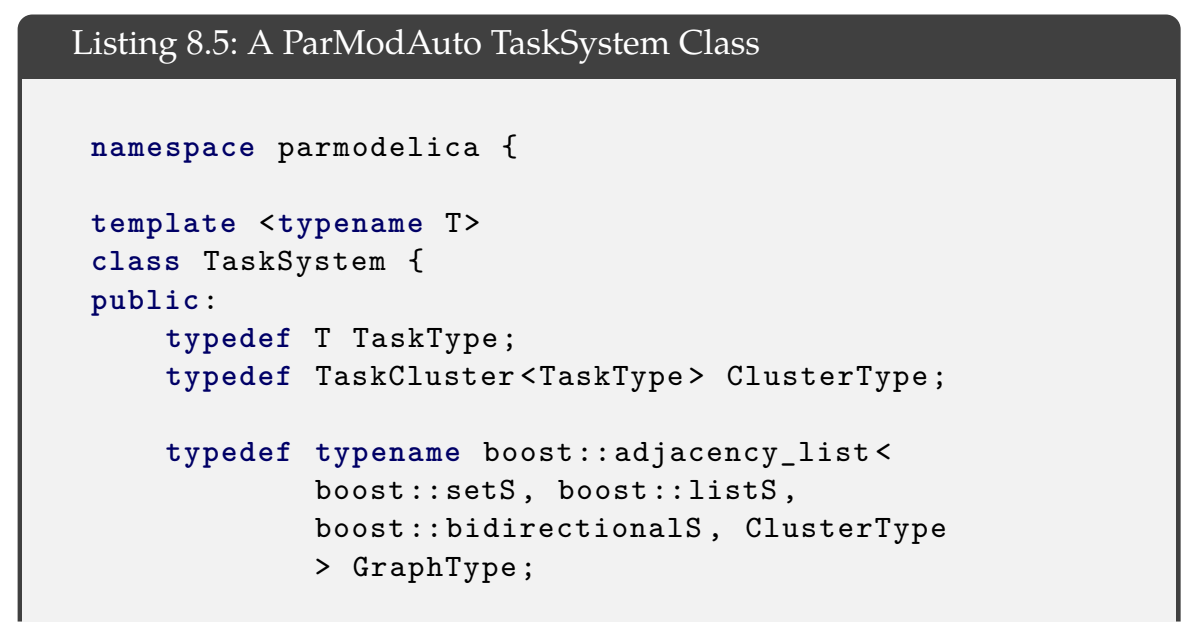

${ }^{1}$ http://www.json.org/ 


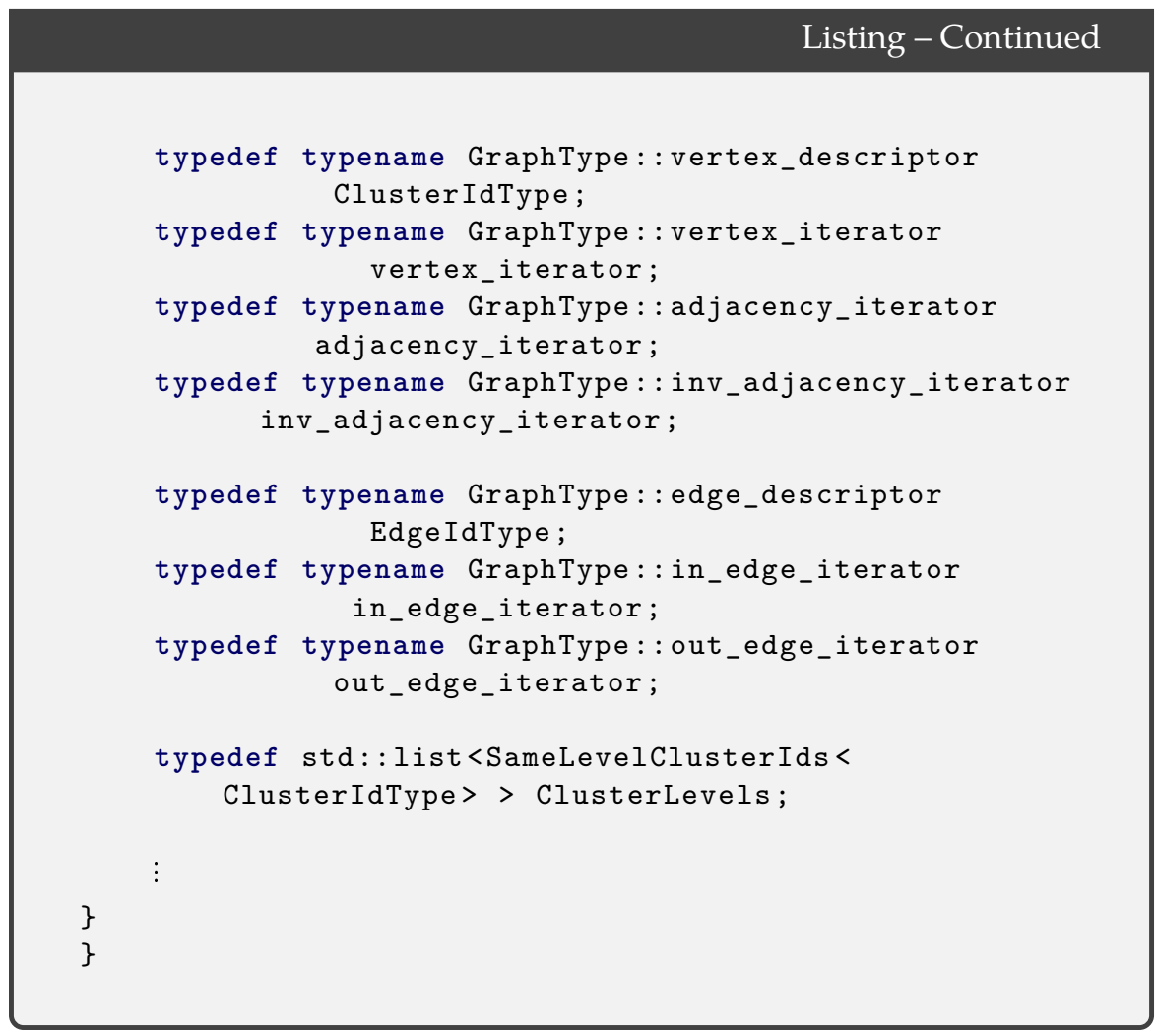

The class provides the necessary routines for traversing and manipulating the graph. For example addition, deletion and merging of different cluster nodes is performed here. In addition, it provides other common functionalities such as computing node levels to be used by the clustering heuristics. The scheduler classes use this class to schedule and execute the task nodes of an equation system.

\subsubsection{Schedulers}

Schedulers in ParModelica can be seen as composed of two layers. The clustering rules that are chosen as suitable for each scheduler can be seen as one layer. These clustering rules are applied in the order they are specified on each rescheduling attempt. The next layer of the scheduler interface is the actual synchronization, control, monitoring, and rescheduling rules/mechanisms. An example level scheduler interface is shown at the end of Listing 8.6. This scheduler specializes the core StepLevels executor with specific clustering rules suitable for generating balanced levels. In a way, the core StepLevels class is responsible for synchronizations and monitoring. This 
separation allows for a customizable interface which can adjust for existing or new clustering rules with existing schedulers.

\section{Listing 8.6: The ParModelica Level Scheduler class}

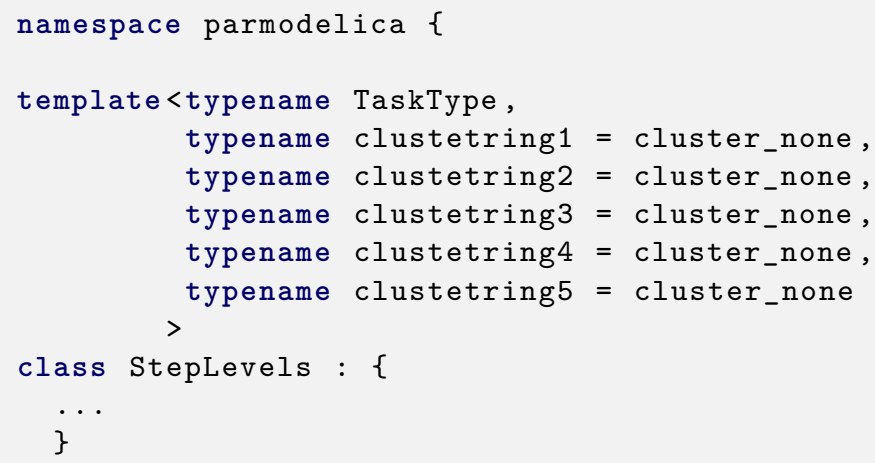

\subsubsection{Equation System Representation}

For a clean and flexible inter-operability with the compiler or tool, a dedicated interface mechanism or approach is needed. ParModAuto achievs this - currently for the OpenModelica Compiler - by providing a dedicated class interface that provides what is, and only what is, relevant and necessary for the task system representation and schedulers to manipulate and execute the equation system effectively.

\section{Listing 8.7: A Model class representing an OpenModelic model}

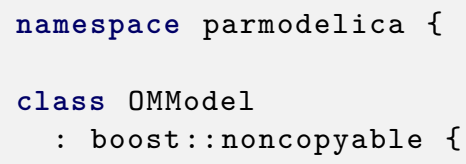


Listing - Continued

typedef Equation::FunctionType FunctionType;

typedef LevelScheduler<Equation> SchedulerT;

// typedef ClusterDynamicScheduler $\langle$ Equation $\rangle$ SchedulerT;

typedef TaskSystem<Equation> TaskSystemT;

private:

std:string model_name;

bool intialized;

DATA* data;

threadData_t* threadData;

public:

OMModel ();

void initialize(const char*, DATA*, threadData_t* , FunctionType*);

FunctionType* ini_system_funcs ;

TaskSystem T INI_system;

SchedulerT INI_scheduler;

FunctionType* dae_system_funcs ;

Tasksystem T DAE_system;

SchedulerT DAE_scheduler;

FunctionType* ode_system_funcs ;

TaskSystemT ODE_system;

SchedulerT ODE_scheduler;

\};

\}

Listing 8.7 shows a simplified model class representing a Modelica model code gnerated by the OpenModelica compiler. This class is what the ParModelica implementation considers as the model. All other details of the actual model simulation relelvant to the OpenModelica runtime system are all kept independent. This interface class provides all that is neccessary - together with the dependency graph - for a parallelized evaluation of either the ODE, DAE, initial system of equations generated by the OpenModelica compiler for the given Modelica model. The OpenModelica runtime crates an instance of and initializes this model class at the start of simulation. Once initialized the 
OpenModelica simulation runtime can invoke the library for a parallelized execution for any the three systems in the Model class above by using the corresponding scheduler.

\subsubsection{Extra Functionalities}

ParModAuto provides timer utility classes for easy use. These timer classes are Operating Systems specific. On Microsoft Windows OS variants it uses the QueryPerformanceCounter Windows API function that provides very high resolution $(<1 \mu \mathrm{s})$ time stamp that can be used for time-interval measurements. On POSIX based systems it uses the Boost Chrono Library's system_clock implementation. These timers are used for profiling the execution cost of each task as well as for general timing needs such as reporting total time consumption, measuring scheduling or clustering overheads and so on.

There are also functionalities for dumping the Directed Acyclic Graphs representing the task graph at any point during the simulation process. Currently there is support for graphs dumps in two different formats: Graphiz and Graphml. These dumps are quite valuable in visualizing the effects of different clustering heuristics that are applied to either the original or the modified task graph during the simulation process. 


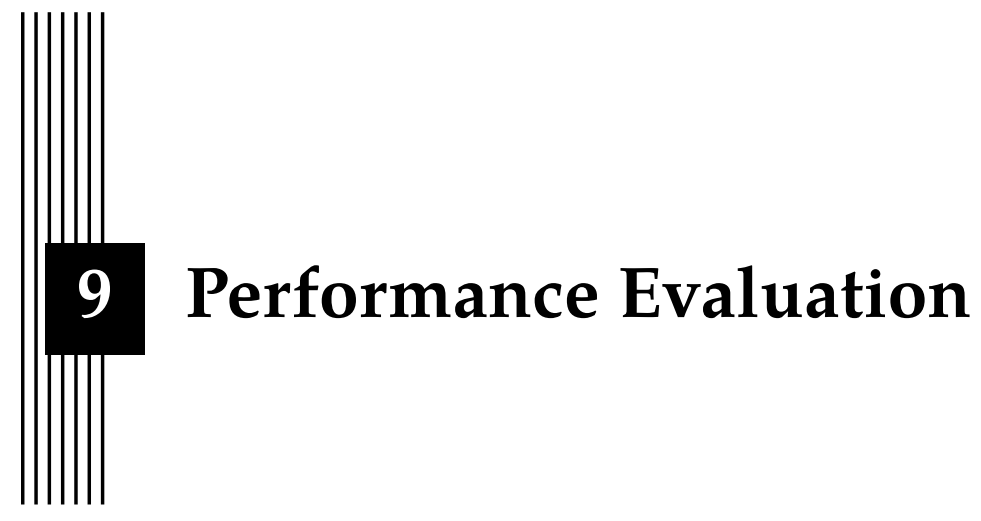

This chapter presents and summarizes results obtained from performance evaluation of the strongly connected component based automatic parallelization approach discussed in the previous three chapters.

\subsection{Overview}

Simulation of a model, at runtime, involves evaluations of multiple stages or components. First there is initialization. This stage involves finding a consistent set of initial values for the state variables. This is more or less a one time operation performed before normal integration steps, i.e. simulation, begins. Although it is possible to parallelize the initialization, since it also involves evaluating a set of equations, it is not included in this performance evaluation.

There are also solvers for linear and non-linear systems. These solvers are used whenever the need arises. If the set of equations for initialization, for example, contains such systems then these solvers are used. This goes the same for the rest of simulation. It is possible to parallelize these solvers too, although the parallelization is mostly data parallel and has little to do with what this work is intended for (See Section 4.3.3).

The main part of a simulation under normal circumstances in the evaluation of the equation system by the integration method. The integration methods require evaluation of the equation system at every time step they take. However, not all equations need be evaluated for the integration meth- 
ods. The set of equations which directly or indirectly affect the values of the state derivatives, i.e. the derivatives of the variables which are considered known, need to be evaluated. This set of equations is referred to as the ODE (for Ordinary Differential Equations). The rest of the equations need only be evaluated at output points or communication points. Output points are where we are interested in actually obtaining the values of the variables.

The evaluation of the ODE is typically the most expensive part of simulation since it accumulates over each time step taken by the integrators or solvers. The ODE might even be evaluated more than once per time step if the integration methods need to do so. Due to this the ODE system is the part that is parallelized in all the performance evaluations presented here. However, it should be noted that parallelizing the other equation systems of the model such as the DAE system or algebraic systems can be performed in exactly the same and straightforward manner.

\subsection{Setup}

The test models used are from libraries that are already available and widely used. Models from two libraries are used: the Modelica Standard Library [8] and the ScalableTestSuite Library [20]. They cover a wide range of fields from Analog Electrical, Digital Electrical, Mechanical, Fluid and Thermal application domains.

These models are not specifically written for this work. More importantly there was no consideration made with regard to the possibility of parallelization by the modellers. It should be noted that the models from the ScalableTestSuite library were intended to analyse the performance of the symbolic processing of Modelica compilers. As such, they were written to be easily scalable by adjusting some parameters. This makes them suitable for parallelization since large systems of organized structures can be generated. The main target here is to see how the implementation performs in libraries that are used on a day to day basis by many modellers.

All tests have been performed on a 64-bit Intel Core $^{\mathrm{TM}}$ i7-3930K CPU @ 3.20GHz machine running Ubuntu 18.04.1 LTS. The processor is a 6-core processor with support for hyper-threading up to 12 threads.

Speedup results are presented for the Level Scheduler and Flow Graph based scheduler. All timing results are averaged from 5 independent simulations for each test model. This means that each graph summarizes results gathered from 30 independent simulations for each test model. All time values are in milliseconds unless specified otherwise. All time values in the tables are also milliseconds.

As mentioned earlier only the ODE system is parallelized. The timing results are only for this portion of the simulation execution. This means that model compilation and other operations such as initialization are not 
included in any of the results. Profiling steps that are needed to collect cost information for tasks are included in the speedup computations since they are integral part of the parallel evaluation. All additional parallelization related operations such as clustering overheads are presented separately for each test case.

All simulations use the default OpenModelica solver, DASSL [88]. Simulation duration - the time duration for which we are interested in simulating, not the execution time - for each model is decided either by experimental annotations that are part of the model or the default OpenModelica simulation duration of 1 second is used. This, as mentioned earlier does not affect the speedup since the parallelization is concerned with each time step separately anyway unless these extra step possibly introduce some disturbance to the system. It should be noted that all models perform more than a thousand evaluations of the ODE. In some cases close to 100000. This means the average evaluation times are computed as an average of these thousands of steps per simulation and then over 5 independent simulations afterwards.

All simulations performed with the level scheduler used the running average based rescheduling policy discussed in Section 7.4.1 with a rescheduling threshold of $20 \%$. This means rescheduling is performed when the current parallel evaluation average is $20 \%$ higher or lower than the average at the last scheduling point.

The clustering heuristics MSP, MLP and MLB (See Section 7.3) are used for evaluations with the Level Scheduler in that specific order. The MLB heuristics is applied for two times the number of threads used for the evaluation. For example, for evaluations with 2 threads, the MLB heuristic is applied to produce 4 bins per level of the task graph. This is done to give the scheduler some dynamic scheduling freedom. It should be mentioned that increasing the number of bins by small amounts does not seem to produce any noticeable degradation in performance.

\subsection{Test Cases and Results}

For each test case model speedup results are presented. The structure of the model is also summarized to give some overview or impression of the expected behaviour. For each model the tables summarize the number of total tasks that the system is dealing with and the number of linear and non-linear blocks out of the total tasks. The average sequential cost of one evaluation of the system with no parallelization, i.e. default simulation with OpenModelica, is presented.

Level scheduler related attributes are also summarized. Each table presents the number of schedules performed during one simulation (this is a median of the 5 runs). The number of schedules performed can be expected to vary to some extent across simulations since no two simulations have exactly 
the same timing measurement. Nonetheless, for simulations on the same machine the number of rescheduling between multiple simulations can be expected to be very close to the values presented here. The average clustering time is also presented. This is a total of the cost of clustering the task system over all rescheduling performed by the simulation. The average profiling cost is presented to demonstrate how expensive profiling steps are compared to sequential evaluation steps with no profiling operations.

\subsubsection{CauerLowPassSC}

The Modelica.Electrical.Analog.Examples.CauerLowPassSC model is a fifth order low-pass-filter. It is made up of nine capacitors and eleven switched capacitors. It is simulated for time duration of 0 to 10 seconds. The structure of the system is summarized in Table 9.1.

The model results in a total of 50 tasks of which 24 are linear systems of equations. Such a system made up any linear-systems is expected to be a good selection for parallelization. The speedup results for this model are shown in Figure 9.1. The obtained speedup is not as big as expected. However, this is acceptable considering that one evaluation of this system takes about $0.078962 \mathrm{~ms}$. Even though it is suited for parallelization, the total execution as a whole, is too low to overcome the influence of the overhead of parallel execution to show the expected level of speedup.

The model is made up of multiple linear systems. Linear systems, as opposed to non-linear systems, are relatively static in the sense that the execution cost of the system does not vary much from execution to execution. However, this model performs as much as 9 reschedules per simulation when using the level scheduler. All of this reschedules happen in the first 40 or so evaluations out of 95000 total evaluations per simulation. The reason for this behaviour is as follows. The first execution of the graph is always sequential since it needs to collect cost information for each task. The second evaluation is, therefore, the evaluation where the threads are invoked for the first time. This first parallel step incurs a bit more extra overhead. Most of the time this is relatively insignificant. For this specific model however, since it has a very small overall execution cost, this first parallel execution is relatively a lot more expensive than the other parallel evaluations. The rescheduling policy used here is the average based rescheduling explain in Section 7.4.1. The running average starts too high due to the relatively expensive first parallel execution and then goes down quickly over the first few subsequent evaluations triggering multiple reschedules. Eventually the average stabilizes and the simulation performs the next 95000 evaluations without any further rescheduling. Although it is possible to avoid these reschedulings by increasing the threshold we have left it as it is to demonstrate how the scheduler adapts to changes. 


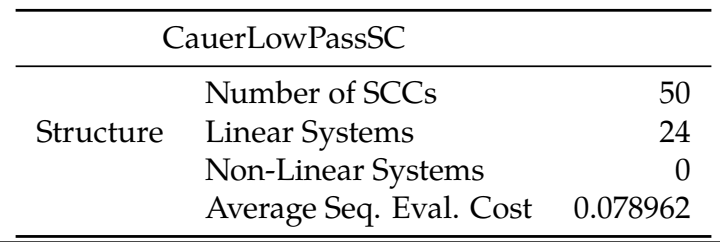

\begin{tabular}{lrrrr}
\hline \multicolumn{4}{c}{ Level Scheduler } & \\
\hline Threads & 2 & 4 & 8 & 12 \\
\hline Schedules & 5 & 9 & 9 & 9 \\
Clustering time & 0.542382 & 0.626609 & 0.391318 & 0.201698 \\
Avg. Profiling Eval. Cost & 0.174001 & 0.172801 & 0.177946 & 0.17298 \\
\hline
\end{tabular}

Table 9.1: Summary of the CauerLowPassSC model

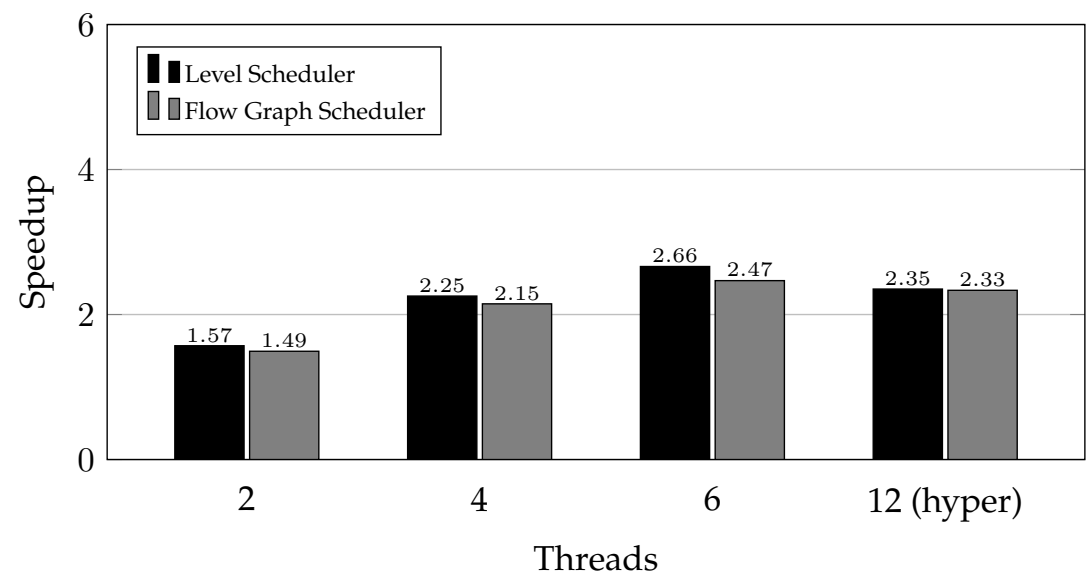

Figure 9.1: Speed-up for CauerLowPassSC model

\subsubsection{BranchingDynamicPipes}

The Modelica.Fluid.Examples.BranchingDynamicPipes model demonstrates the use of distributed pipe models with dynamic energy, mass, and momentum balances. It is simulated for the duration of 0 to 20 seconds. The structure of the BranchingDynamicPipes model is summarized in Table 9.2 and the speedups obtained are presented in Figure 9.2.

The structure of this model is suitable for parallelization. Even though the total sequential time needed for one evaluation is just $1.26 \mathrm{~ms}$, the system is made up of a few large linear and non-linear systems which are relatively expensive compared to the rest of the tasks. This structure allows for a good parallelization efficiency. 


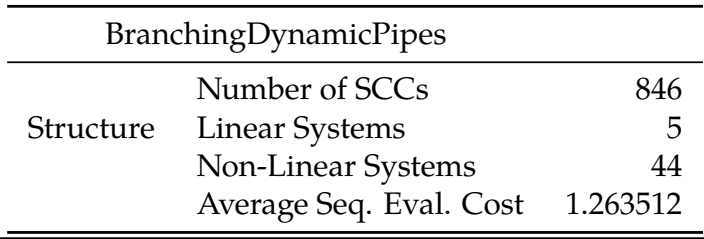

\begin{tabular}{lrrrr}
\hline \multicolumn{5}{c}{ Level Scheduler } \\
Threads & 2 & 4 & 8 & 12 \\
\hline Schedules & 3 & 3 & 4 & 5 \\
Clustering time & 5.99407 & 5.67078 & 6.79137 & 7.24449 \\
Avg. Profiling Eval. Cost & 2.53521 & 2.46265 & 2.29693 & 2.34305 \\
\hline
\end{tabular}

Table 9.2: Summary of the BranchingDynamicPipes model

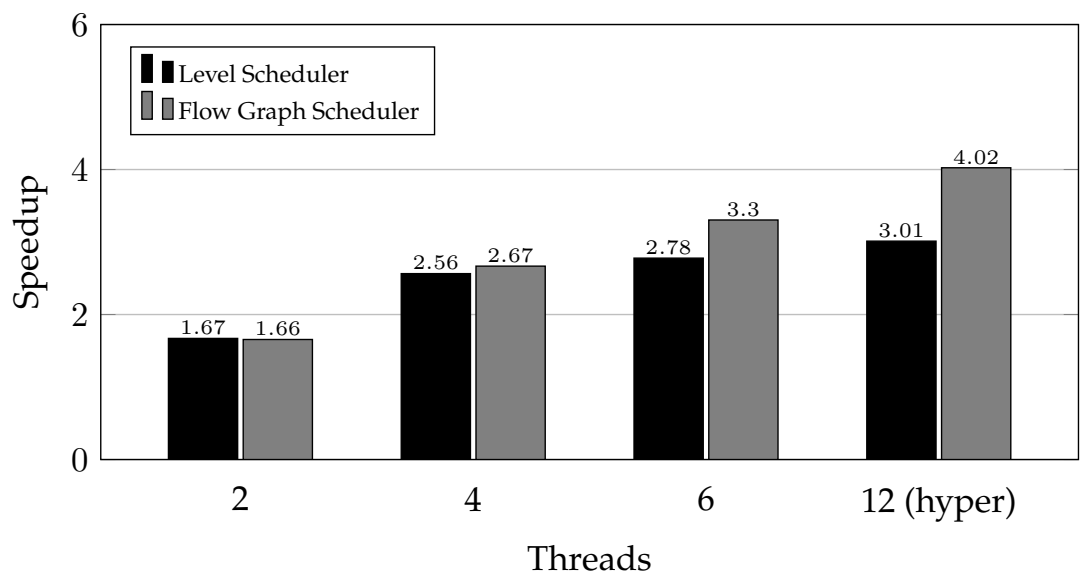

Figure 9.2: Speed-up for BranchingDynamicPipes model

\subsubsection{Spice3BenchmarkFourBitBinaryAdder}

The Modelica.Electrical.Spice3.Examples.Spice3BenchmarkFourBitBinaryAdder model is a digital adder circuit to add two four bit binary numbers. The model is built out of two two-bit adders which respectively are built out of two one bit adders. Each one-bit adder is built out of nine NAND circuits.

It is simulated for the duration of 0 to $10^{-8}$ seconds. Although the end time is set to a very low value the model is very expensive in terms of total simulation time because the integrator takes very small time steps leading to a large number of ODE evaluations. Regarding the cost of each evaluation, the average cost of a single sequential evaluation of the ODE takes $0.632 \mathrm{~ms}$ making it a less expensive than the BranchingDynamicPipes 


\begin{tabular}{llr}
\hline \multicolumn{3}{l}{ Spice3BenchmarkFourBitBinaryAdder } \\
\hline \multirow{4}{*}{ Structure } & Number of SCCs & 1130 \\
& Linear Systems & 104 \\
& Non-Linear Systems & 72 \\
& Average Seq. Eval. Cost & 1.118201 \\
\hline
\end{tabular}

\begin{tabular}{lrrrr}
\hline \multicolumn{5}{c}{ Level Scheduler } \\
\hline Threads & 2 & 4 & 8 & 12 \\
\hline Schedules & 2 & 4 & 3 & 4 \\
Clustering time & 9.70882 & 17.2434 & 6.48198 & 7.32118 \\
Avg. Profiling Eval. Cost & 4.09241 & 4.255 & 3.11138 & 3.05862 \\
\hline
\end{tabular}

Table 9.3: Summary of the Spice3BenchmarkFourBitBinaryAdder model

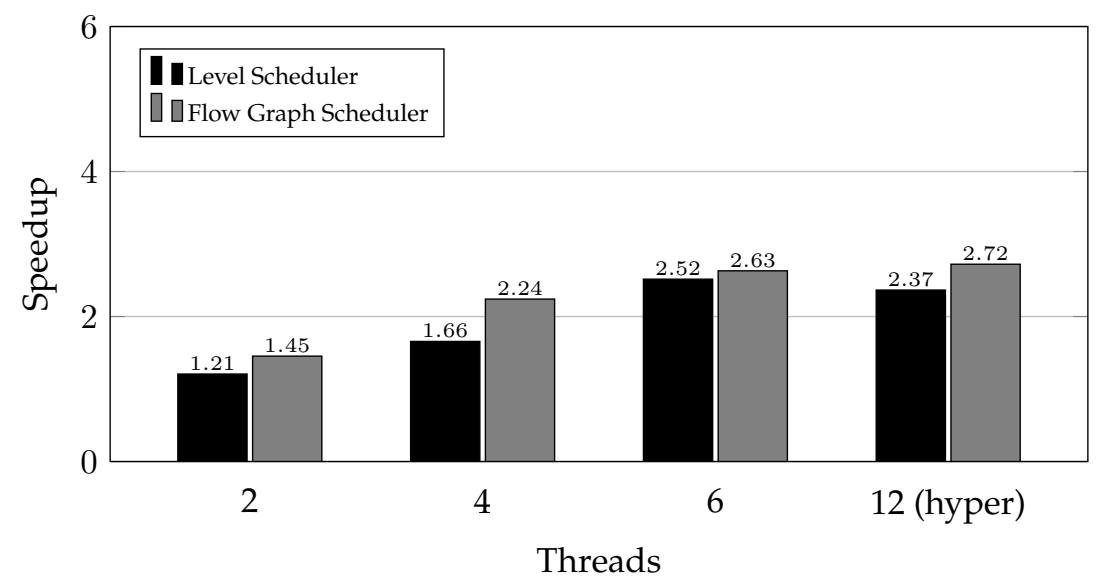

Figure 9.3: Speed-up for Spice3BenchmarkFourBitBinaryAdder model

model even though it has more tasks than the latter. The structure of Spice3BenchmarkFourBitBinaryAdder model is summarized in Table 9.3 and the speedups obtained are presented in Figure 9.3.

\subsubsection{EngineV6}

The Modelica.Mechanics.MultiBody.Examples.Loops.EngineV6 model is an engine made up of 6 cylinders arranged in $\mathrm{V}$ formation. The model belongs to a set of examples in the Loops package. These are example models to demonstrate the formation of large coupled linear or non-linear systems in the Mechanical modelling domains. 


\begin{tabular}{llr}
\hline & EngineV6 & \\
\hline \multirow{4}{*}{ Structure } & Number of SCCs & 420 \\
& Linear Systems & 14 \\
& Non-Linear Systems & 12 \\
& Average Seq. Eval. Cost & 0.280323 \\
\hline
\end{tabular}

\begin{tabular}{lrrrr}
\hline \multicolumn{5}{c}{ Level Scheduler } \\
Threads & 2 & 4 & 8 & 12 \\
\hline Schedules & 2 & 2 & 2 & 2 \\
Clustering time & 1.95484 & 1.68847 & 0.890957 & 0.300035 \\
Avg. Profiling Eval. Cost & 1.07373 & 1.16913 & 1.06109 & 1.02173 \\
\hline
\end{tabular}

Table 9.4: Summary of the EngineV6 model

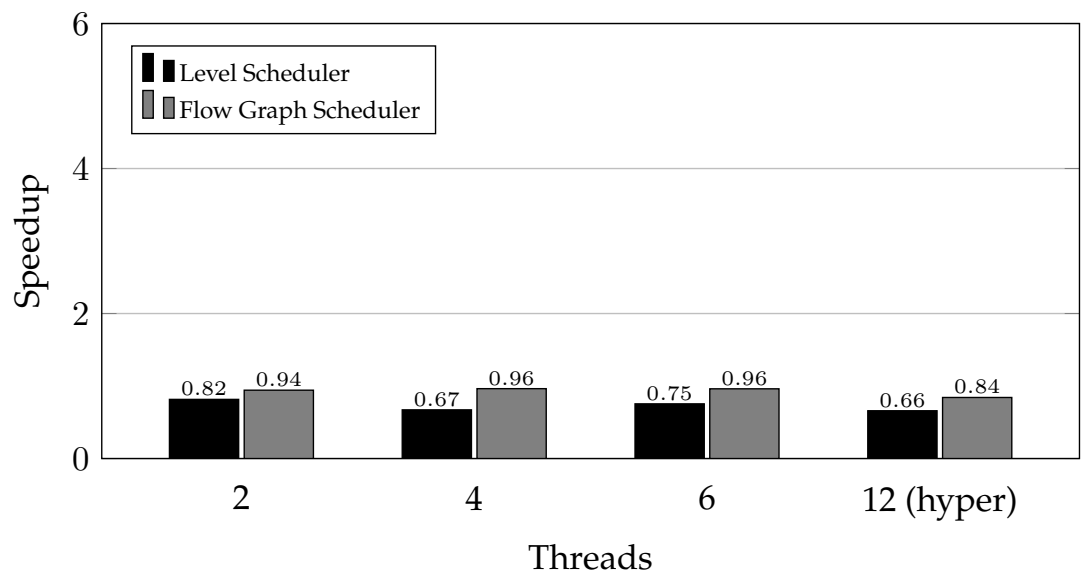

Figure 9.4: Speed-up for EngineV6 model

It is simulated for the duration of 0 to 1.01 seconds. The structure of the EngineV6 model is summarized in Table 9.4 and the speedups obtained are presented in Figure 9.4. Here parallelization seems to degrade performance across the board. This is as a result of increased overhead due to parallelization related processing while there is virtually nothing to benefit from. Even if the system has 420 tasks in total the 12 linear and non-linear systems dominate most of the execution. To make matters worse these large systems are heavily dependent on each other. This means the potential to evaluate them in parallel is non-existent. As far as the level scheduler is concerned, any further clustering operations performed on this task system will have little to no significance since the levels are dominated by the large chained tasks anyway. 


\subsubsection{SteamPipe}

These models are from the ScalableTestSuite Library [20] are designed to evaluate the performance of different Modelica compilers and simulation tools in scalable manner. The library is intended for analysing the performance and scalability of multiple operations of a typical Modelica tool such as flattening of object-oriented structures, symbolic processing operations such as causalization, and runtime simulation performance when faced with large models.

The models in this library can be scaled in size by modifying a few parameters. Test models from the Thermal library of this library are used to evaluate the performance of the parallelization when faced with large scale models. The selected ScalableTestSuite.Thermal.Advection.ScaledExperiments.SteamPipe model presents detailed thermal advection model with thermal expansion effects using IF97 water vapour. The model shows the mass, energy, and momentum balance equations for 1D flow of steam in a pipe, using the finite volume method.

Parallelized simulations of these models is performed with increasing sizes to see how parallelization performance responds to very large number of tasks. The library has scaled versions of the SteamPipe model each with different number of nodes. These example models, namely SteamPipe_N_320, SteamPipe_N_640, SteamPipe_N_1280 and SteamPipe_N_2560 are used for evaluation. The speedups gained from simulations for these models are presented in Figure 9.5, Figure 9.6, Figure 9.7, and Figure 9.8. The corresponding structures for each model can be seen in Table 9.5, Table 9.6, Table 9.7, and Table 9.8 respectively.

Even the smallest of these four models has a higher sequential evaluation cost than the models from the Modelica Standard Library presented earlier. This increase in size can be seen to translate well into gains for parallelization. The overhead of all the tracking and scheduling related operations starts to become less of a factor as the model sizes get bigger. These models also benefit from the fact that they are scaled versions of the base model. Scaling the size of models in such manner by simply increasing the number of components inside it while keeping the overall structure, i.e. the relationship between equations, more or less regular means that the resulting task graph can be balanced and/or scheduled conveniently.

Despite the fact that these sample models are regular in structure even on increased sizes, they demonstrate that the parallelization can be sufficiently effective once the models start getting bigger. As we can see in Figure 9.8 the parallelized simulations achieved around 4 times speed-up with 6 threads in a 6 core machine. Speedup of upto 5.5 times is obtained with hyper-threading signifying that there is room for scaling on processors with more cores as long as the models are past a certain size.

The example Modelica Standard Library models are small. They are designed to demonstrate the uses of Modelica. Even the models that we con- 


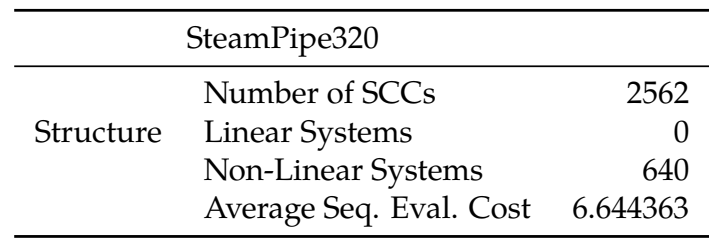

\begin{tabular}{lrrrr}
\hline \multicolumn{5}{c}{ Level Scheduler } \\
\hline Threads & 2 & 4 & 8 & 12 \\
\hline Schedules & 1 & 1 & 1 & 2 \\
Clustering time & 5.49261 & 5.61183 & 5.7761 & 12.1992 \\
Avg. Profiling Eval. Cost & 11.8376 & 11.7815 & 12.0153 & 12.3414 \\
\hline
\end{tabular}

Table 9.5: Summary of the SteamPipe320 model

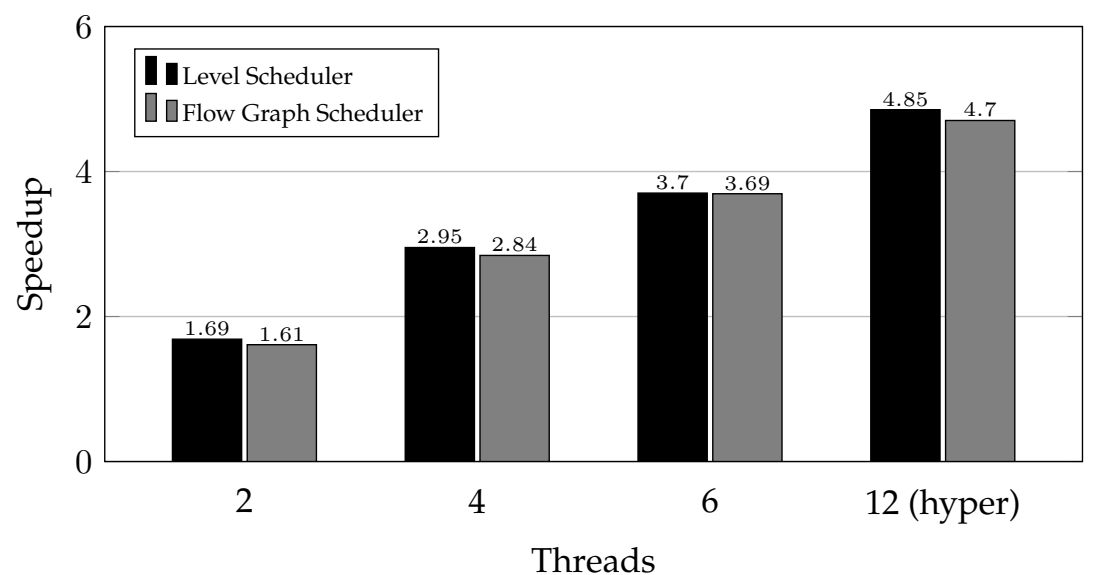

Figure 9.5: Speed-up for SteamPipe320 model

sider big, such as Spice3BenchmarkFourBitBinaryAdder or EngineV6 are not "expensive" if we are looking at each evaluation individually instead of the whole simulation (they cost 1.118 and 0.28 milliseconds per evaluation respectively). This is what parallelization is dealing with. Each step individually. The point is that actual real life models that are used by companies and professional users can get considerably bigger than the examples from the Modelica Standard Library. These models can benefit from parallelization even to a higher extent as demonstrated by the large ScalableTestSuite models. 


\begin{tabular}{|c|c|c|c|c|c|}
\hline \multicolumn{5}{|c|}{ SteamPipe640 } & \\
\hline Structure & $\begin{array}{l}\text { Num } \\
\text { Linea } \\
\text { Non- } \\
\text { Avere }\end{array}$ & $\begin{array}{l}\text { ber of SCC } \\
\text { r Systems } \\
\text { Linear Sys } \\
\text { ge Seq. E }\end{array}$ & $\begin{array}{l}\text { s } \\
\text { tems } \\
\text { al. Cost }\end{array}$ & $\begin{array}{r}5122 \\
0 \\
1280 \\
13.637166\end{array}$ & \\
\hline \multicolumn{6}{|c|}{ Level Scheduler } \\
\hline \multicolumn{2}{|l|}{ Threads } & 2 & 4 & 8 & 12 \\
\hline \multicolumn{2}{|l|}{ Schedules } & 1 & 1 & 1 & 1 \\
\hline \multicolumn{2}{|c|}{ Clustering time } & 11.8925 & 11.6056 & 12.0607 & 12.7789 \\
\hline \multicolumn{2}{|c|}{ Avg. Profiling Eval. Cost } & 24.0635 & 24.1303 & 23.9011 & 23.7406 \\
\hline
\end{tabular}

Table 9.6: Summary of the SteamPipe640 model

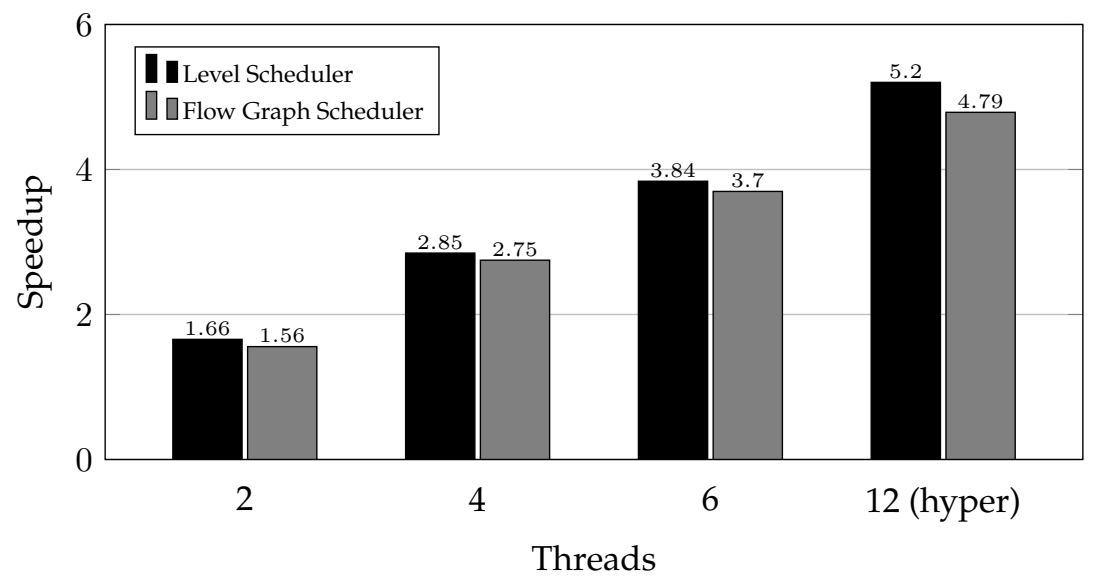

Figure 9.6: Speed-up for SteamPipe640 model 


\begin{tabular}{|c|c|c|c|c|c|}
\hline \multicolumn{5}{|c|}{ SteamPipe1280 } & \\
\hline Structure & $\begin{array}{l}\text { Num } \\
\text { Line } \\
\text { Non- } \\
\text { Aver }\end{array}$ & $\begin{array}{l}\text { oer of SCC } \\
\text { r Systems } \\
\text { inear Sys } \\
\text { ge Seq. E }\end{array}$ & $\begin{array}{l}\text { s } \\
\text { al. Cost }\end{array}$ & $\begin{array}{r}10242 \\
0 \\
2560 \\
27.404762\end{array}$ & \\
\hline \multicolumn{6}{|c|}{ Level Scheduler } \\
\hline \multicolumn{2}{|l|}{ Threads } & 2 & 4 & 8 & 12 \\
\hline \multicolumn{2}{|l|}{ Schedules } & 1 & 1 & 1 & 2 \\
\hline \multicolumn{2}{|c|}{ Clustering time } & 32.3829 & 28.777 & 30.4327 & 63.0318 \\
\hline \multicolumn{2}{|c|}{ Avg. Profiling Eval. Cost } & 47.8801 & 47.7594 & 48.0284 & 49.1183 \\
\hline
\end{tabular}

Table 9.7: Summary of the SteamPipe1280 model

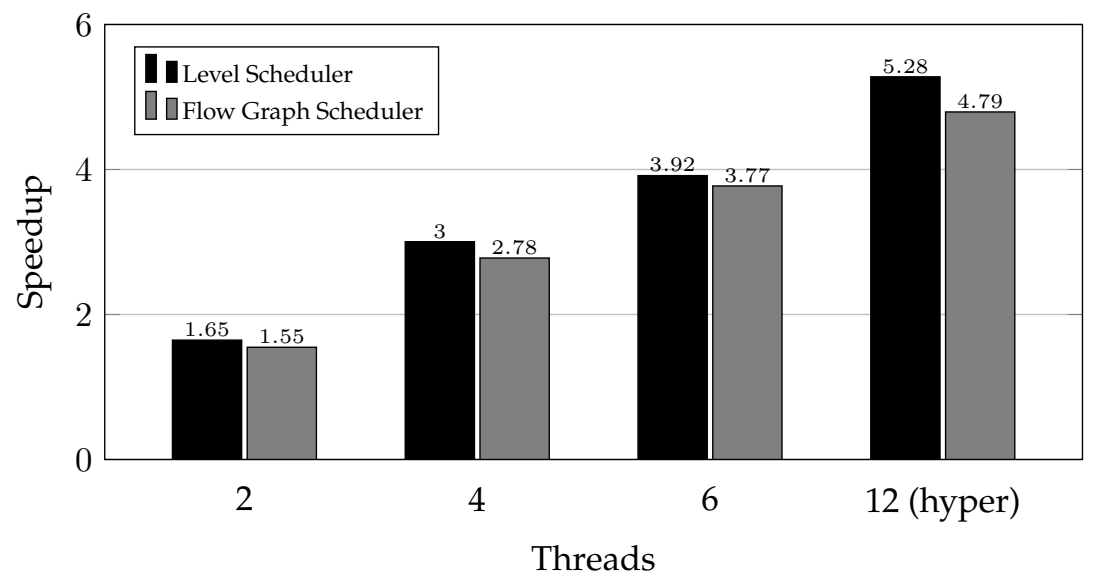

Figure 9.7: Speed-up for SteamPipe1280 model 


\begin{tabular}{|c|c|c|c|c|c|}
\hline \multicolumn{5}{|c|}{ SteamPipe2560 } & \\
\hline Structure & $\begin{array}{l}\text { Num } \\
\text { Line } \\
\text { Non- } \\
\text { Aver }\end{array}$ & $\begin{array}{l}\text { er of SCC } \\
\text { r Systems } \\
\text { inear Sys } \\
\text { ge Seq. E }\end{array}$ & $\begin{array}{l}\text { s } \\
\text { tems } \\
\text { al. Cost }\end{array}$ & $\begin{array}{r}20482 \\
0 \\
5120 \\
54.629950\end{array}$ & \\
\hline \multicolumn{6}{|c|}{ Level Scheduler } \\
\hline Threads & & 2 & 4 & 8 & 12 \\
\hline Schedules & & 1 & 2 & 1 & 1 \\
\hline Clustering time & & 74.8512 & 156.677 & 78.4642 & 80.2086 \\
\hline Avg. Profiling Eval & Cost & 94.8692 & 96.8807 & 96.2456 & 95.0243 \\
\hline
\end{tabular}

Table 9.8: Summary of the SteamPipe2560 model

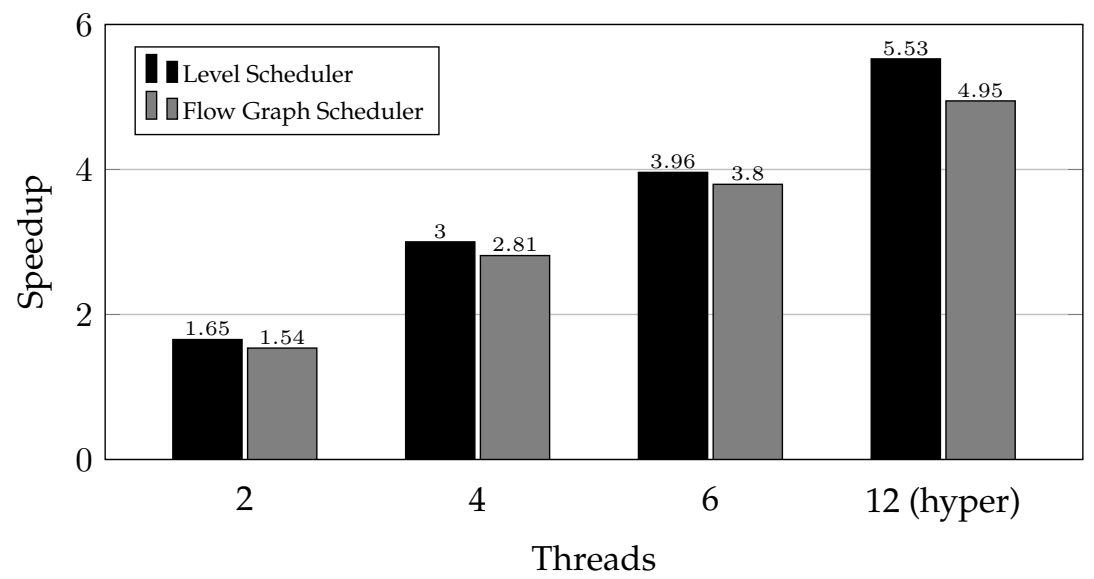

Figure 9.8: Speed-up for SteamPipe2560 model 



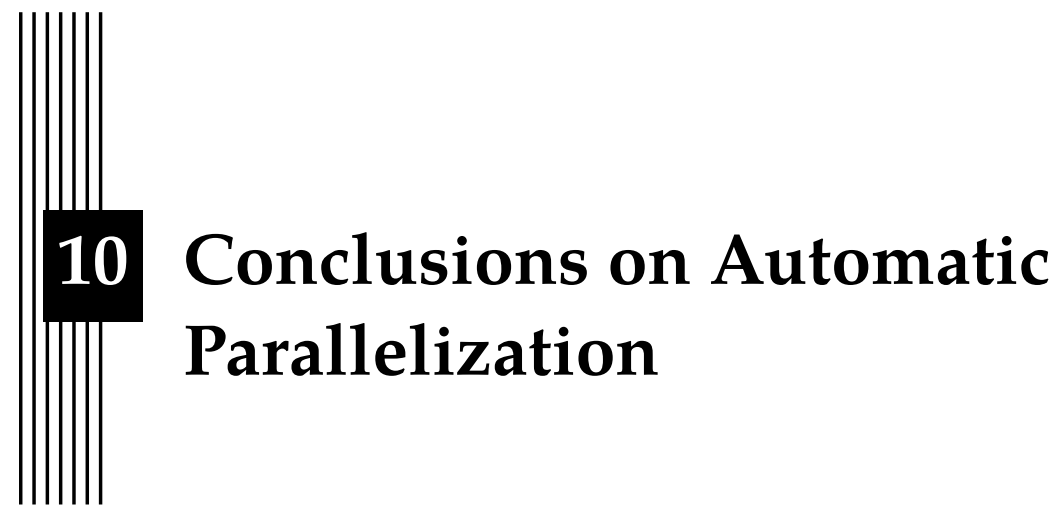

Parallelization of equation systems is feasible. As the models get bigger and bigger the benefit of parallelization will be even more prevalent. We have investigated two approaches of automatic parallelization of equation systems. The first approach is based on analysing and utilizing connected component structures in equation systems generated from modelling and simulation languages showed some benefits. It can be a feasible approach when certain amounts of decoupling can be expected in the physical model. Furthermore, this approach can be utilized even better with specific modelling techniques such as employing delayed systems when applicable. A thread safe memory management for the OpenModelica runtime was designed and implemented for this approach as well. It provided a low overhead and simple parallelization scheme.

However, this approach also suffered from certain limitations. It was limited by design to static cost estimations which meant that it could not adapt to dynamically changing loads of simulations. In addition, it is not applicable for a wide range of domains without modifications to existing models. In the cases where specific design techniques are applicable this still means that the models will not resemble the original system as much as they can if designed without such considerations.

In order to address these limitations a parallelization approach based on strongly connected components has investigated. This provides a convenient and widely applicable parallelization scheme that can deal with more complicated and highly connected equation systems. Different clustering heuristics 
and scheduling approaches are investigated and implemented. Furthermore, the approach was based on runtime processing. This allows for a more responsive design that can adjust to the dynamic behaviour of task systems with adaptive runtime scheduling and rescheduling capabilities.

The ParModAuto Task System parallelization library provides all the processing related to parallelization. All the needed functionalities such construction of task systems, profiling and cost estimations, clustering and scheduling and monitoring are provided in simple high-level stand-alone library that can be easily plugged into existing tools and frameworks. It is also designed to be flexible and easily extendible giving users the freedom to experiment with new approaches with ease.

ParModAuto is sufficiently high level to be used without requiring advanced knowledge in parallel programming. It is also designed with portability in mind so that it can be usable by multiple simulation tools. It can save time and effort for developers, researchers or tools looking to implement either a complete task parallelization implementation or try out different scheduling approaches

The performance of the strongly connected approach has been investigated within the OpenModelica Modeling and Simulation Environment. Tests have been performed with models from multiple domains such as Electrical, Mechanical and Thermal areas. The test cases demonstrate a considerable amount of performance improvement. Furthermore, the benefit of parallelization in this approach is going to get better as models get larger. 
Part II

Explicit Parallelization 



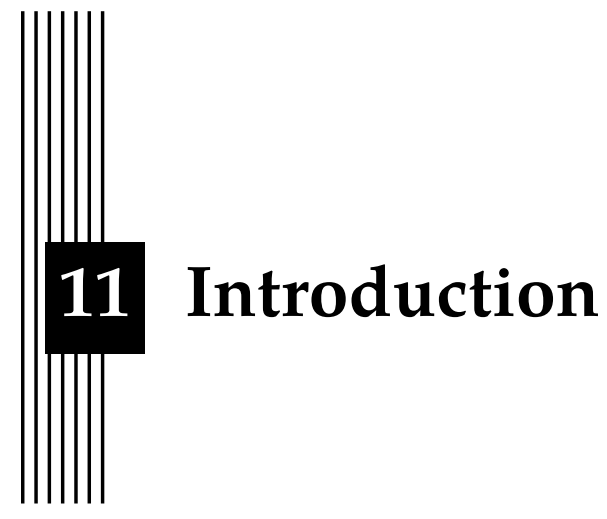

As mentioned earlier most previous work regarding parallel execution support in the OpenModelica compiler has been focused on automatic parallelization where the burden of finding and analysing parallelism has been put on the compiler. In this work, however, this responsibility is left to the end user programmer. The compiler provides additional high level language constructs needed for explicitly stating parallelism in the algorithmic part of the modeling language. This among others includes parallel variables, parallel functions, kernel functions and parallel for loops indicated by the parfor keyword. There are also some target language specific constructs and functions (in this case for OpenCL). All these extensions are collectively called ParModelica Extensions. These will all be presented in this chapter.

\subsection{General Purpose Graphic Processing Unit (GPGPU) programming}

A GPGPU is a general purpose Graphics Processing Units (GPUs) designed for use in data-parallel graphic as well as non-graphic computations. Traditionally the use of most GPUs has been limited to processing of only graphics data. However, in recent years it has become more common to use them also for processing of non-graphic scientific and engineering computations.

GPGPU programming is based on the concept of using the CPU and GPU as heterogeneous computing units. The CPU is used to execute serial parts of 
the computation and manage the GPU whereas the GPU is used as another highly parallel processing unit to perform parallel parts of the computation. Different frameworks of programming for GPUs are available now. OpenCL, CUDA, DirectX [78], OpenGL [63] and DirectCompute [77] are some examples. The last three frameworks are more focused on the traditional use of using GPUs for processing of graphic data. However CUDA and OpenCL provide rather complete support for proper GPGPU programming. These two are used widely to implement non-graphic heavy computations.

\subsection{OpenCL}

\subsubsection{The OpenCL Architecture}

OpenCL is the first open, royalty-free standard for cross-platform, parallel programming of modern processors found in personal computers, servers and hand-held/embedded devices. The OpenCL programming language is based on C99 with some extensions for parallel execution management. By using OpenCL it is possible to write parallel algorithms that can be easily ported between multiple devices with minimal or no changes to the source code. The framework is composed of the OpenCL; programming language, API, libraries and a runtime system to support software development. The OpenCL framework can be divided into a hierarchy of models: Platform Model, Memory model, Execution model and Programming model. A brief description of these models is given in the following sections. However, for a complete understanding of the OpenCL framework it is recommended that the reader accesses [64].

\subsubsection{Platform Model}

The OpenCL platform model is defined as a Host processor connected to one or more OpenCL devices. The OpenCL devices are divided into one or more Computing Units (CU) which in turn are divided into one or more Processing Elements (PE). The host is responsible for managing the executions on OpenCL devices. This management includes: identifying and initializing OpenCL devices, data copy operations and submitting parallel jobs to the OpenCL device.

\subsubsection{Execution Model}

The execution of an OpenCL program consists of two parts. The Host program which executes on the host processor and the OpenCL program which executes on the OpenCL device. The host program manages the execution of the OpenCL program. An OpenCL program is a collection of kernels which execute as separate and independent programs. Kernels are executed simul- 
taneously by all threads specified for the kernel execution. The number and mapping of threads to Computing Units of the OpenCL device is handled by the host program. Each thread executing an instance of a kernel is called a work-item. Each thread or work-item has unique id to help identify it. A work-item can have additional id fields depending on the arrangement specified by the host program. Work-items can be arranged into work-groups. Each work-group has a unique id. Work-items are assigned a unique local id within a work-group so that a single work-item can be uniquely identified by its global id or by a combination of its local id and work-group id. The workitems in a given work-group execute concurrently on the processing elements of a single compute unit.

A wide range of programming models can be mapped onto this execution model. OpenCL explicitly supports two of these models; the data parallel programming model and the task parallel programming model.

\subsubsection{Memory Model}

The OpenCL memory space is divided into four parts:

- Global Memory: This memory region permits read/write access to all work-items in all work-groups. Work-items can read from or write to any element of a memory object. Reads and writes to global memory may be cached depending on the capabilities of the device.

- Constant Memory: A region of global memory that remains constant during the execution of a kernel. The host allocates and initializes memory objects placed into constant memory.

- Local Memory: A memory region local to a work-group. This memory region can be used to allocate variables that are shared by all work-items in that work-group. The local memory space maybe implemented as dedicated regions of memory on the OpenCL device. Alternatively, it may be mapped onto sections of the global memory.

- Private Memory: A region of memory private to a work-item. Variables defined in one work-item's private memory are not visible to another work-item.

This division of memory spaces is shown in Figure 11.1. ${ }^{1}$. The access and allocation rights of the host and kernels to these memory spaces are shown in Table 11.1.

\footnotetext{
${ }^{1}$ Picture taken from [64]
} 


\section{Figure 11.1: OpenCL Memory Model.}

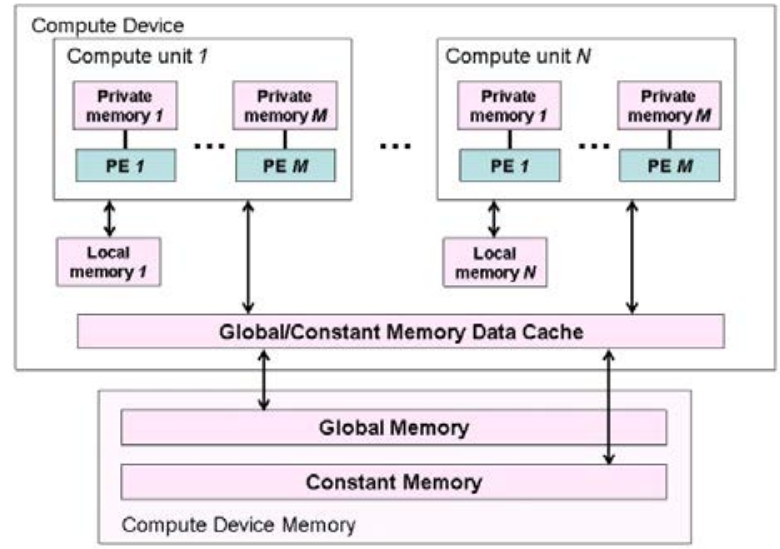

\begin{tabular}{lllll}
\hline & Global & Constant & Local & Private \\
\hline \multirow{2}{*}{ Host } & Dynamic alloc. & Dynamic alloc & Dynamic alloc. & No alloc. \\
& Read/Write & Read/Write & No access & No access \\
\hline \multirow{2}{*}{ Kernel } & No alloc. & Static alloc. & Static alloc. & Static alloc. \\
& Read/Write & Read-only & Read/Write & Read/Write \\
\hline
\end{tabular}

Table 11.1: OpenCL allocation and memory access capabilities

\subsubsection{Programming Model}

The OpenCL execution model supports data parallel and task parallel programming models, as well as supporting hybrids of these two models. The primary programming model driving the design of OpenCL is data parallel. The data parallel programming model defines a computation in terms of a sequence of instructions applied to multiple elements of a memory object. In a strictly data parallel model, there is a one-to-one mapping between the work-item and the element in a memory object over which a kernel can be executed in parallel. OpenCL implements a relaxed version of the data parallel programming model where a strict one-to-one mapping is not a requirement.

OpenCL provides a hierarchical data parallel programming model. There are two ways to specify the hierarchical subdivision. In the explicit model a programmer defines the total number of work-items to execute in parallel and also how the work-items are divided among work-groups. In the implicit model, the programmer only specifies the total number of work-items to exe- 
cute in parallel, and the division into work-groups is managed by the OpenCL implementation.

The OpenCL task parallel programming model defines a model in which a single instance of a kernel is executed independent of any index space. It is logically equivalent to executing a kernel on a Compute Unit with a workgroup containing a single work-item. Under this model, users can express parallelism by:

- Using vector data types implemented by the device,

- Enqueing multiple tasks, and/or

- Enqueing native kernels developed using a programming model orthogonal to OpenCL.

\subsection{Modelica for Scientific Computing}

A rather overlooked application area of Modelica is its usability in general scientific computations. The language, as it is right now, is quite well suited to be used in heavy scientific computations and not just in modeling areas. Most scientific computation algorithms involve linear algebra operations on large amounts of data usually organized as vectors, matrices, and higher dimensional arrays. Modelica provides a very powerful array representation and related features that can make writing these complicated algorithms more convenient. It is of course not possible to cover all the features Modelica provides for convenient algorithmic code implementation here. However, this section presents a few selected features that can give a general idea of what Modelica has to offer for the scientific computation community.

Modelica arrays can be declared with unknown sizes, specified by colons as dimension sizes, as shown in Listing 11.1. Here the size of the array $x$ which is the input to the function unknowInputSizeArray is not specified. The actual size is of this input array is determined at call time by the argument passed to it, as demonstrated by the calls in the function callMultiple. The output array y of the function is also flexible. Its size is determined by the size of the first dimension of the input array via the call size $(x, 1)$.

\section{Listing 11.1: Unknow size arrays}

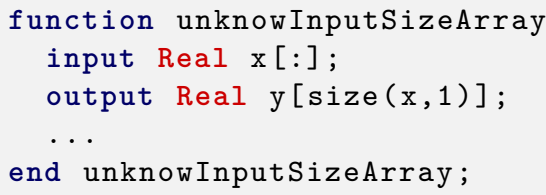




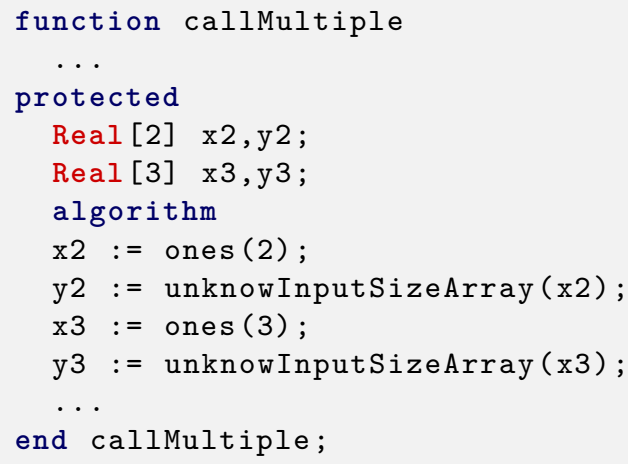

This kind dynamic adjustment of array sizes makes functions more generic and reusable. For example without the ability to work with unknown size arrays we would need one function for each size to perform the same operation.

Indexing and Slicing: there are many of ways to index Modelica arrays. Indexing an array can result in a scalar or an array of selected elements of the original array. The latter is what we call array slicing. Simple examples of array slicing operations are shown in Listing 11.2.

\section{Listing 11.2: Array slicing}

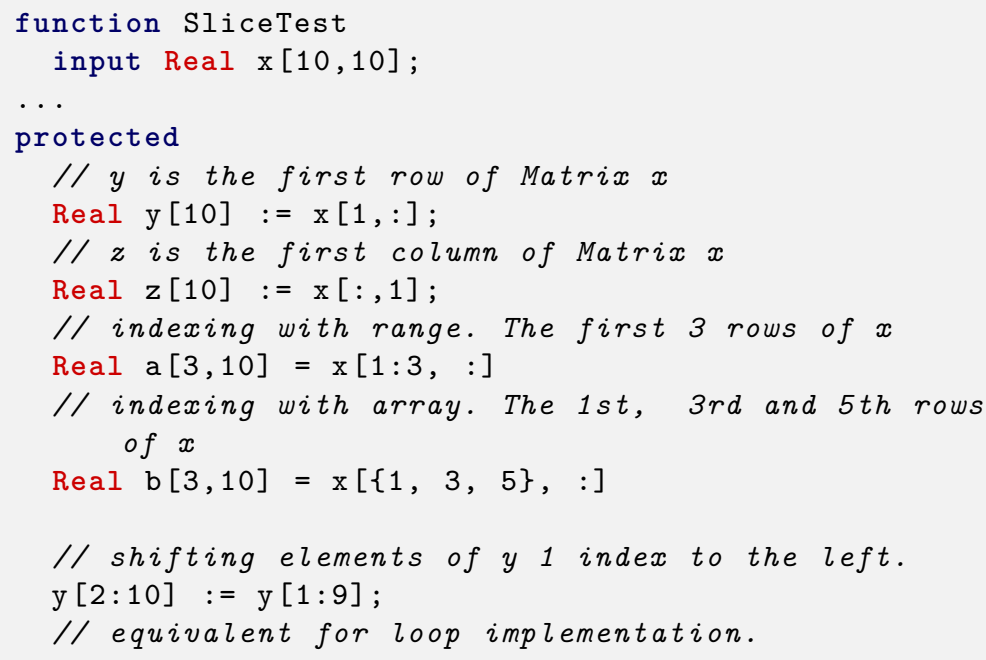


Listing - Continued

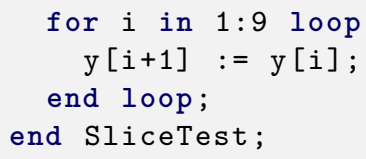

Slicing allows algorithms to be more concise and provides the opportunity to take advantage of code structure to generate a more efficient code. More complex usages of slicing than shown in these examples enable modellers/programmers to manipulate arrays in a convenient way.

Modelica overloads the normal built-in arithmetic operators $(+,-,,, /, \wedge)$ for vector, matrix and array arithmetical operations. Depending on the type of operands involved in the arithmetic operations these operators are resolved to the specific mathematical operation. For example

$$
C=A * B
$$

is resolved as matrix multiplication if $\mathrm{A}$ and $\mathrm{B}$ are matrices and the multiplication dimensions match $(m \times n * n \times k)$ and results in matrix $\mathrm{C}(m \times k)$. This kind of resolving of operators is done for more combinations of operands. The full list can be found in [9].

In addition to overloading the common arithmetic operators, Modelica also provides a set of element-wise operators for arrays. These operators operate on an element by element basis on arrays of matching dimensions and dimension sizes. For example

$$
C=A . * B
$$

is an element by element multiplication of $\mathrm{A}(m \times n)$ and $\mathrm{B}(m \times n)$ which results in the matrix $\mathrm{C}(m \times n)$. The full list and semantic rules of the element-wise operators can be found in [9].

Modelica also has support for range expressions and reduction operations for concise and readable representation of algorithms. Consider that we want to compute the sum of the first $n$ odd numbers starting from 1 . That is

$$
S=\sum_{i=1}^{n}(2 * i-1)
$$

Using for ranges expressions and the built-in sum reduction operator we can write this in Modelica as shown in Listing 11.3. 
Listing 11.3: Reduction operations

$S=\operatorname{sum}(2 i-1$ for $i$ in $1: n)$

In addition to these array and range related features the object oriented features of Modelica can help to further simplify scientific computation algorithms. For example records (which, in some contexts, are the Modelica equivalent of $\mathrm{C}++$ classes) with operator overloading can be used to manipulate structured data sets in a convenient manner. A good example of this usage is the Complex numbers library from the Modelica Standard Library.

There are many more simple and advanced features that make Modelica very suitable for algorithms of scientific computations. Yet it seems like Modelica has been rather overlooked by the scientific computation community so far. This might be due to two main reasons:

- Modelica is originally intended for physical system modeling. Hence the focus of the user community is more on what it has to offer for modeling. Being such a powerful language for modeling has somehow over shadowed its convenience and power in the other areas. Even modelers who use Modelica frequently seem to use other languages e.g. Matlab when they have the need to do some sort of complex scientific computation.

- With regard to scientific computations Modelica has a crippling lack of library support. The only substantial library for scientific computations that is available is the Modelica.Math library which mostly provides interfaces to external LAPACK routines. This lack of library support might be the main reason why even frequent Modelica users prefer other languages and tools for their computations.

The explicit data-parallel programming extensions presented in this work provide an even further improvement on how Modelica can handle complex heavy computations on modern multi-core and multi-processor architectures.

\subsection{Related work}

There hasn't been as much research focused on explicit parallelism around Modelica as automatic approaches. One implementation that offered the potential for explicit parallel programming was the NestStepModelica [61] that was based on NestStep [60] targeting BSP (Bulk-Synchronous Parallel) computation model which is an abstraction of a restricted message passing architecture and charges cost for communication. 
The focus of the work presented here is on parallelizing executions for highly data parallel SPMD (Single Program, Multiple Data) architectures. It generates OpenCL code for parallel algorithms. OpenCL was given priority over CUDA because of its portability. Generating OpenCL code ensures that simulations can be run with parallel support on OpenCL enabled Graphics and Central Processor Units (GPU and CPU). This includes many multi-core CPUs from Intel [55] and Advanced Micro Devices (AMD) [3] as well as a range of GPUs from NVIDIA [83] and AMD (for a complete list of supported devices see [62]). However explicit CUDA code generation is also planned to be supported and the current implementation provides most, if not all, constructs needed for CUDA code generation and execution as well. 



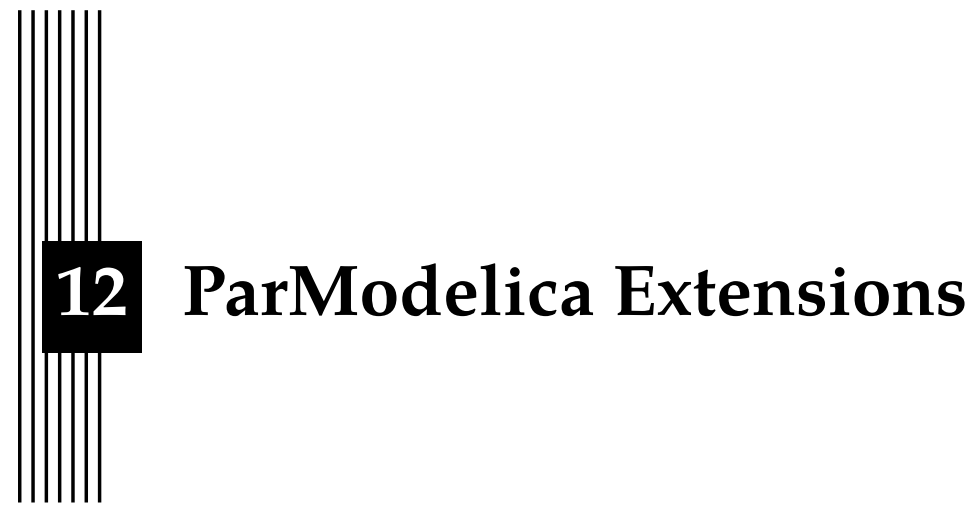

In the past years many high level programming languages have started introducing support for explicit parallel programming. MATLAB's Parallel Toolbox [76] (at 10,000 SEK for individual licence), Mathematica's (a.k.a The Wolfram Language) Parallel Computing [92] [93], and GNU Octave's Parallel Package [85] are some examples.

In Modelica the only way to utilize parallel computing capabilities of the prevalent multi-core and processor architectures has so far been limited to $e x$ ternalization. User have to write their parallel implementations in frameworks such as OpenMP, CUDA or OpenCL and then wrap these implementations in one of the languages that Modelica has external function support for, e.g. C. Then they have to provide the corresponding external functions in their Modelica code and use them. The mechanism of multi-step external function would be complicated and error prone even for experienced programmers familiar with multi-threaded programming and low level languages. For Modelica users, modellers who are mostly application and domain engineers, this task is even more daunting. Pointers, dynamic memory management and other low level concepts are not the strength of modellers in high level languages. They will end up spending more time in debugging and fixing mundane errors instead of focusing on their algorithms and models.

The ParModelica extensions are a set of constructs introduced to the Modelica language to enable describing of multithreaded execution. Instead of achieving parallelism through library and built-in functions the ParModelica extensions are designed to be integrated part of the language. For example 
Figure 12.1: Pre-ParModelica vs ParModelica parallel programming in Modelica

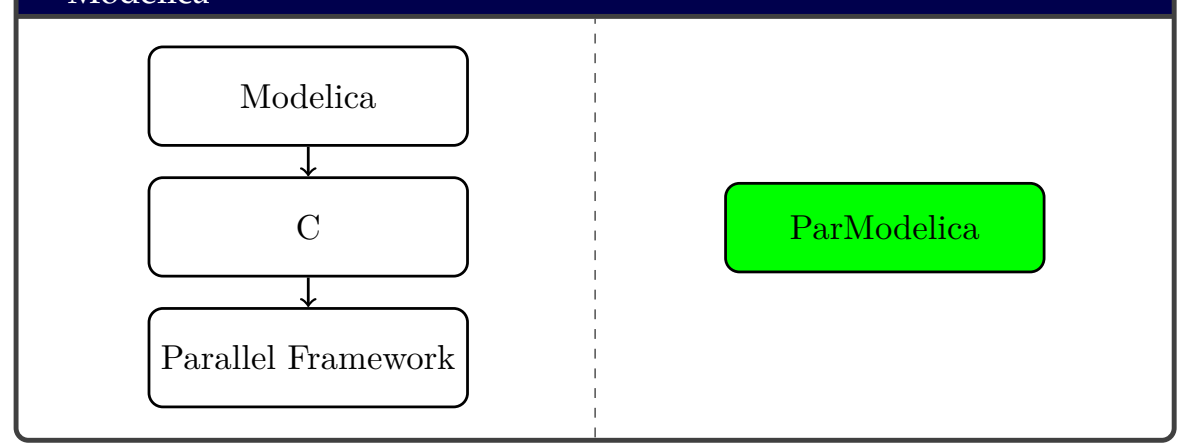

memory mappings of variables is treated as an extra attribute of a variable just like constness or visibility. This means that describing parallel programs fits very well with the rest of the language constructs. In addition, it allows the compiler to perform better syntactic and semantic checks.

\section{Listing 12.1: ParModelica vs MATLAB GPU variables}

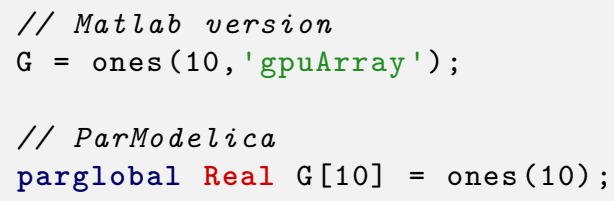

These extensions are designed to be very similar to parallel programming frameworks/languages such as OpenCL and CUDA. This is intended to make sure that users familiar with these frameworks can easily transition to the Modelica implementations. Staying close to these rather popular frameworks means that new programmers can to some extent refer to documentations, methods and discussions originally intended for those languages. Furthermore, they will be able to translate implementations originally done in OpenCL/CUDA to the ParModelica version without much difficulty.

There are a few of programmability and usage differences compared to the base OpenCL extensions. These are explained in the sections for their respective counter-parts. 


\subsection{Parallel Variables}

\section{Listing 12.2: ParModelica device variables}

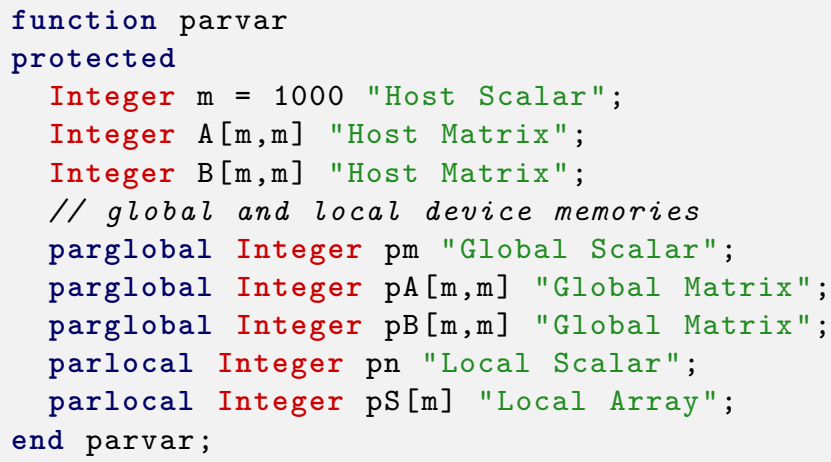

Parallel variables are variables allocated in the memory space of the device used for parallel computation. OpenCL code can be executed on host CPU as well as on GPUs whereas CUDA code executes only on GPU. Since the OpenCL and CUDA enabled GPUs use their own local (different from CPU) memory for execution all necessary data should be available on the specific device's memory. Even when running OpenCL computations on CPU the variables used for parallel execution need to be explicitly stated so that the OpenCL drivers and APIs can handle them properly.

ParModelica parallel variables are declared simply by preceding the variable declaration with the corresponding keyword for the intended memory space. The implementation currently does not support allocations or accesses to the constant mmory space, i.e., only global variables identified by parglobal and local variables identified by parlocal are allowed.

\begin{tabular}{|c|c|}
\hline $\mathrm{A}:=\mathrm{B}$ & Serial assignment. \\
\hline $\mathrm{pA}:=\mathrm{A}$ & $\begin{array}{l}\text { Copy from host memory }(A) \text { to device global memory }(p A) \text {. write } \\
\text { operation }\end{array}$ \\
\hline $\mathrm{B}:=\mathrm{pB}$ & $\begin{array}{l}\text { Copy from device global memory }(p B) \text { to host memory }(B) \text {. read } \\
\text { operation }\end{array}$ \\
\hline $\mathrm{pA}:=\mathrm{pB}$ & $\begin{array}{l}\text { Copy from one device global memory }(p B) \text { to other memory space } \\
\text { on the same device }(p A) \text {. }\end{array}$ \\
\hline $\begin{array}{l}\mathrm{pm}:=\mathrm{m} \\
\mathrm{n}:=\mathrm{pm} \\
\mathrm{pn}:=\mathrm{pm}\end{array}$ & Scalar versions of the above three assignments. \\
\hline
\end{tabular}

Table 12.1: Parallel Variable Assignment Operation 
Usage of these parallel variables is shown in Listing 12.2. The first three variables are allocated in the host memory. The last four variables are allocated in the corresponding memory space of the device used for parallel execution. In OpenCL case this can be the host CPU itself or any available GPU.

These parallel variables can be passed between functions as arguments. Copying data between host and parallel device memory is as simple as assigning the variables to each other. The compiler and the runtime system handle the details of the copy operation. The assignments shown in Table 12.1 would all be valid in the function shown above.

Parallel variables can only be declared inside a serial function. Variables in kernel (Section 12.3) and parallel functions (Section 12.2) without the qualifiers parglobal or parlocal are allocated as private variables.

Any computational algorithmic statements involving parallel variables should be in either in parallel for loops, parallel functions or kernel functions. These include arithmetic operations on scalar parallel variables and indexing of parallel arrays. Assignments are allowed anywhere in the algorithmic section of Modelica. The compiler performs these semantic checks and reports any such uses.

\subsection{Parallel Functions}

ParModelica parallel functions in this implementation correspond to OpenCL functions defined in kernel files or CUDA's device functions. These are functions available independently to every thread executing on a device. Parallel functions in ParModelica are defined in the same way as normal Modelica functions except that they are preceded by the parallel keyword as shown in Listing 12.3.

\section{Listing 12.3: ParModelica parallel functions}

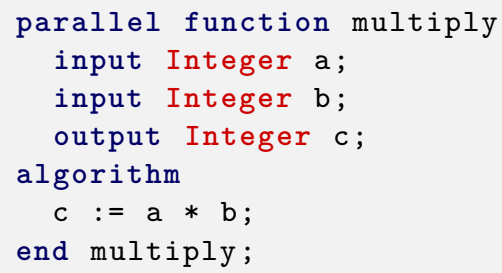


The code for parallel functions is generated in the target language for parallel execution. In the current implementation OpenCL code is generated. Parallel functions have some constraints

- They cannot have parallel for loops in their algorithm sections.

- They can only call other parallel functions or supported built-in functions.

- Recursion is not allowed.

- They can only be called from a body of a parfor loop or from kernel functions, i.e., they are not directly accessible to serial parts of the algorithm.

All of these semantic checks are performed by the compiler and reported.

\subsection{Kernel Functions}

\section{Listing 12.4: ParModelica kernel functions}

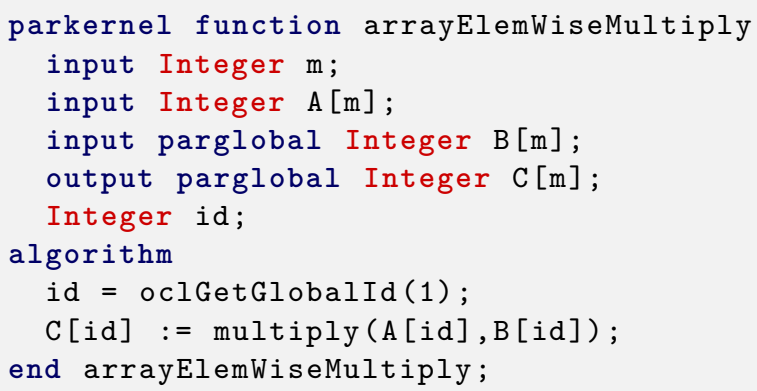

Kernels functions correspond to OpenCL and CUDA kernel functions and global functions respectively. These are entry functions to execution on a device. They can be called from serial parts of Modelica code to start parallel execution on a parallel device. Kernel functions are independently executed by every thread.

ParModelica kernel functions are defined in the same way as normal functions except that they are preceded by the parkernel keyword. A possible implementation example is shown in Listing 12.4. multiply is the parallel function listed in Listing 12.3. The special built-in utility function oclGetGloballd is discussed in Section 12.5.1. The number of threads to be used for the kernel execution can be set by using the function oclSetNumThreads also discussed in Section 12.5.1. This function should be called before any kernel call if the number of threads and their dimensional 
arrangement is needed to be different than the default behavior. Otherwise the default number of threads will be used to execute the kernel function which maximum number of threads of the parallel execution device in a one dimensional arrangement. The implementation supports full three dimensional arrangement of work groups and threads.

There are some constraints on usages of ParModelica kernel functions :

- They cannot have parfor loops in their algorithm body.

- They can only call parallel functions or supported built-in functions. They cannot call other kernel functions.

- They cannot be called from a body of parfor loop or from other kernel functions.

\subsection{Parallel For Loop: parfor}

ParModelica parallel for loops are syntactically very similar to normal for loops with some additional constraints on the body of the loop. These constraints are needed to make sure the iterations can be run simultaneously and independently without any specific order while giving the desired result, i.e., no loop-carried dependencies from one iteration to the next. A Modelica parallel for loop is identified by the keyword parfor as shown in Listing 12.5. multiply is the parallel function listed in Listing 12.3.

The iterations of a parfor loop are equally distributed among available processors. If the range of the iteration is smaller than or equal to the number of threads the parallel device supports, each iteration will be done by a separate thread. If for example our device supports 1024 threads and the loop has 512 iterations then 512 threads will be launched and will each execute a separate iteration. If the number of iterations is larger than the number of threads available then some threads might perform more than one iteration. If for example we have a loop with 768 iterations and a device with a 512 thread limit then 512 threads will be launched which will execute iterations 1 to 512 . The remaining 256 iterations will be done by the first 256 threads out of the 512 as a second step. In future enhancements parfor will be given the extra feature for specifying the desired number of threads explicitly instead of automatically launching threads as described above.

\section{Listing 12.5: ParModelica parallel for loops}

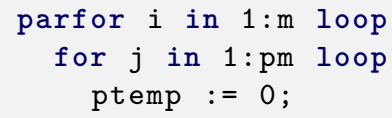




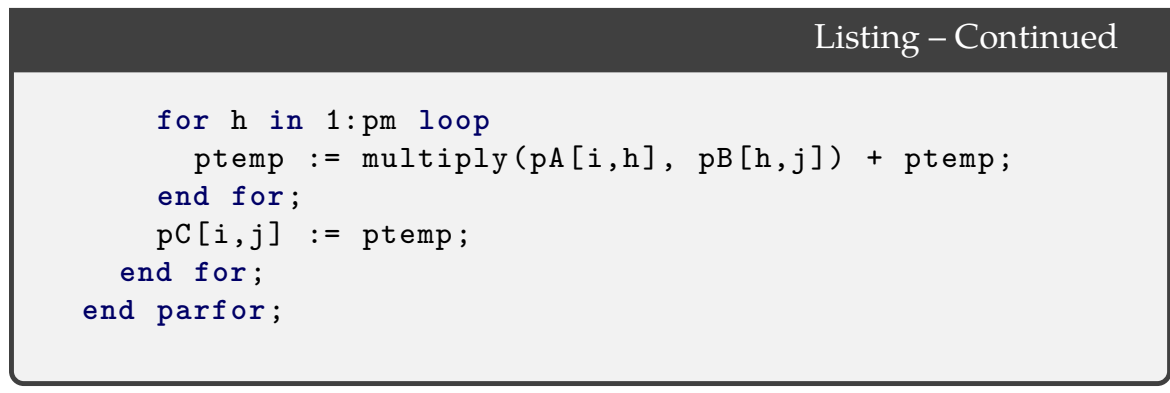

The choice of target architecture and language has put some constraints on parfor loops.

- All variable references in the loop body must be to parglobal variables.

- Iterations should not be dependent on other iterations : no loop-carried dependencies.

- All function calls in the body should be to parallel functions or supported built-in functions only.

- The iterator of a parallel for loop must be of integral type.

- The start, step and end values of a parallel for loop iterator should be integral types.

The first constraint is needed since OpenCL executions can be run on another device than the host CPU where the rest of the simulation code is being executed. To make sure that desired data is made available in the device memory before start of parallel execution this rule must be obeyed. If for example OpenMP has been used for the parallel execution then we would not need this constraint since OpenMP code always runs on the CPU with threads accessing CPU shared memory. There is a reason why the compiler does not automatically detect and copy all variables used or referenced in the loop body. Even if it would be reasonable to automatically copy all needed variables to the device memory, which variables should be copied back? Copying all variables back after the execution of the parfor loop means that potentially unnecessary copy operations would have to be performed. In addition this gives the programmer a better control over the rather expensive memory operations.

\subsection{Built-in Functions}

Some built-in functions have been extended to accept parallel variables as arguments. Accepting parallel arguments means that the computations of the function will be performed on the parallel execution device instead of a single 
thread on the host CPU. The return values from these extended parallel builtin functions are currently only parallel variables. For example consider the built-in function transpose which is used to compute the transpose of a matrix. If a serial matrix is passed to this function as argument the computation will be done on the host CPU and a serial matrix is returned. However if a parallel matrix is given as argument then the computation will be done in parallel on the available device. The return variable will be a parallel variable.

The serial/parallel combination of arguments/return values should be diversified in the future to give more options for the programmer. The compiler should detect the types assigned to return variables and handle any necessary copying automatically. The rules set above on serial/parallel arguments/return-values combination are not hard rules. They are more of choice of implementation and might change in the future. However according to the current implementation any built-in function call involving parallel arguments will return parallel variables.

\subsubsection{Synchronization and Thread Management}

A number of functions related to Synchronization and thread management are also available. These functions are very similar to the OpenCL work-item function (see [web:workitemfuncs]). These functions are:

- oclSetNumThreads $(\ldots, \ldots)$ : is used to specify the number of threads to be used for a kernel function execution. The implementation supports full three dimensional arrangement work groups and items. The function is overloaded for each corresponding dimensional arrangement and should only be called from inside a serial function. It should be called prior to any kernel function call if the number of threads is to be specified for the kernel. Otherwise the kernels will execute with the default number of threads which is the supported maximum. This function is also overloaded to take just one Integer argument. In this case the given integer value will specify the number of threads or work-items to be launched. The actual arrangement of these threads into work-groups will be decided automatically by the OpenCL runtime system. This usage can be seen in Listing 12.6.

- oclGetWorkDim() : returns the number of dimensions used in thread arrangement.

- oclGetGlobalSize (Integer) : returns the total number of threads currently executing the function or kernel. This function should only be called from inside a parallel function or kernel function.

- oclGetLocalSize (Integer) : returns the total number of threads in the work-group of the calling thread in the given dimension. This function should only be called from inside a parallel function or kernel function. 
- oclGetGlobalId (Integer) : returns the global id of the calling thread in the given dimension. This function should only be called from inside a parallel function or kernel function.

- oclGetLocalId(Integer) : returns the local id of the calling thread in the given dimension. This function should only be called from inside a parallel function or kernel function.

- oclGetNumGroups (Integer) : returns the number of work groups in the given dimension. This function should only be called from inside a parallel function or kernel function.

- oclGetGroupId(Integer) : returns the work group id of the calling thread in the given dimension. This function should only be called from inside a parallel function or kernel function.

- oclGlobalBarrier() : will either flush any variables stored in local memory or queue a memory fence to ensure correct ordering of memory operations to local memory of the parallel device. corresponds to OpenCL barrier (CLK_GLOBAL_MEM_FENCE). This function can only be called from inside a parallel function, kernel function or body of parfor loop.

- oclGLocalBarrier () : is used to queue a memory fence to ensure correct ordering of memory operations to global memory of the parallel device. corresponds to OpenCL barrier (CLK_LOCAL_MEM_FENCE). This function can only be called from inside a parallel function, kernel function or body of parfor loop.

\subsubsection{Extra OpenCL Functionalities}

Automatically generated code might not always be as efficient as a manually written code. If the need arrives for a finer control over operations like data distribution and synchronization built-in functions are available for compiling and executing user-written OpenCL code directly from another source file.

- oclbuild (String): This function takes only one String argument. The argument is the name of the OpenCL source file to be built. It returns an integer (type defined as cl_program for clarity) which is used as an id of the built program. This id is used in consequent calls to refer to this OpenCL program. Users can have as many as 10 files built in the same Modelica code (10 within scope) at a time. This limit can be increased in the future. It is just assumed to be enough. 
- oclkernel (oclprogram, String): This function takes two arguments. The first one is the id (Integer) of the OpenCL program built by a previous call to oclbuild. The second argument is the name the kernel or function in that specific program which the user wants to create a kernel for. Users can create as many as 10 kernels at any time. This function also returns an Integer (type defined as cl_kernel) for the same reason as oclbuild.

- oclsetargs (oclkernel,...) : This function is used to set arguments to an OpenCL kernel. It takes a variable number of arguments. However the first argument should be the id of the kernel to be executed (an Integer or cl_kernel). After the first argument a variable number of parallel variables follow. These are the actual arguments to the OpenCL kernel. This function does not return anything.

- oclexecute (oclkernel): This function is used to execute a kernel. It takes the id of the kernel as an argument. After executing the kernel the user can copy back any of the arguments attached to the kernel earlier to obtain just the desired results.

Users can declare OpenCL programs as cl_program and kernels as cl_kernel. These types are just normal type definitions of Integer made just for clarity purposes. They are included with built-in functions so they can be used readily any time. A simple usage of theses utility functions is shown in Listing 12.6. The OpenCL kernel function can perform any operation as long as the arguments match.

\section{Listing 12.6: Loading and executing external OpenCL kernels}

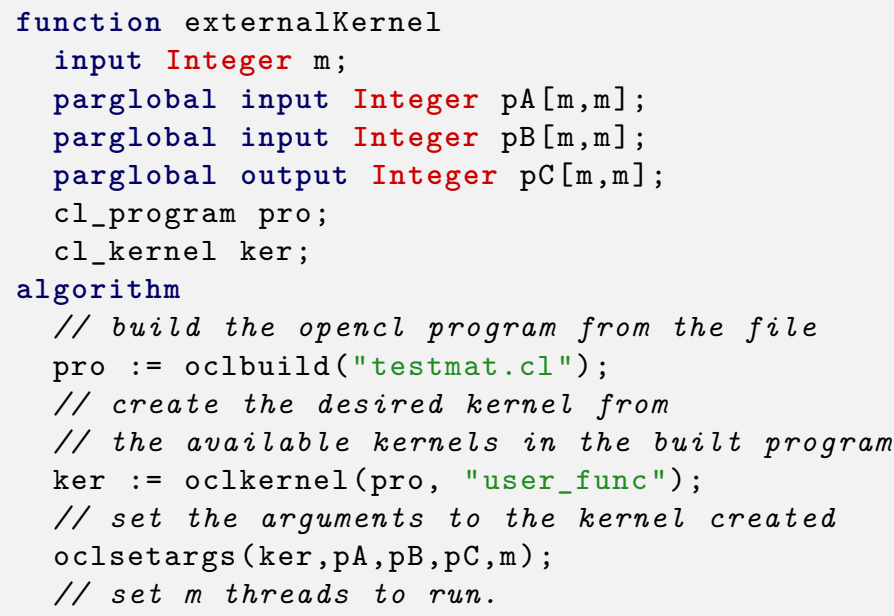




\section{Listing - Continued}

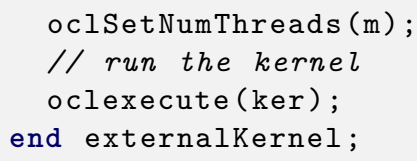

All of the above operations are synchronous in OpenCL jargon. They will return only when the specified operation is completed. Further functionality is planned to be added to these functions to provide better control over execution. 



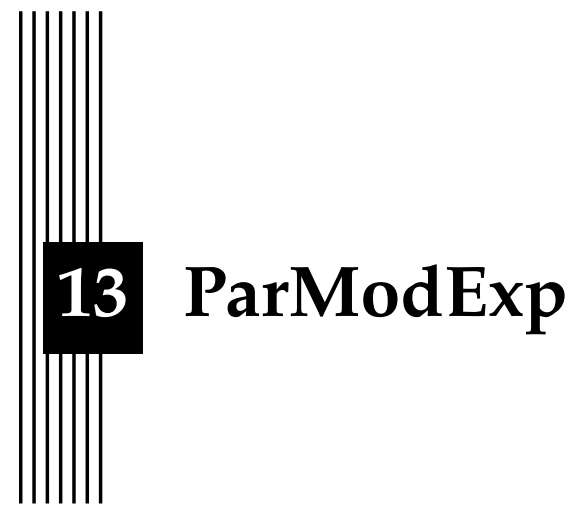

The ParModelica OpenCL runtime provides support for execution and interoperation of the generated OpenCL code and the existing OpenModelica runtime system. The runtime system implementation mainly consists of the ParModelica OpenCL-C runtime library and the ParModelica OpenCL utility headers.

\subsection{ParModelica OpenCL-C Runtime Library}

The OpenCL-C runtime library provides the mechanisms for connecting the OpenCL device execution and the host serial $\mathrm{C}$ execution. The library provides a number of functionalities which allow the OpenModelica normal runtime system and the generated OpenCL code work seamlessly.

\section{Listing 13.1: ParModelica device array}

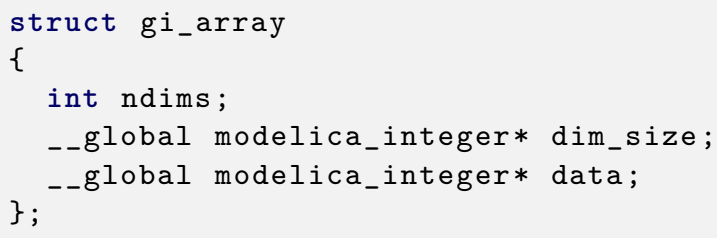




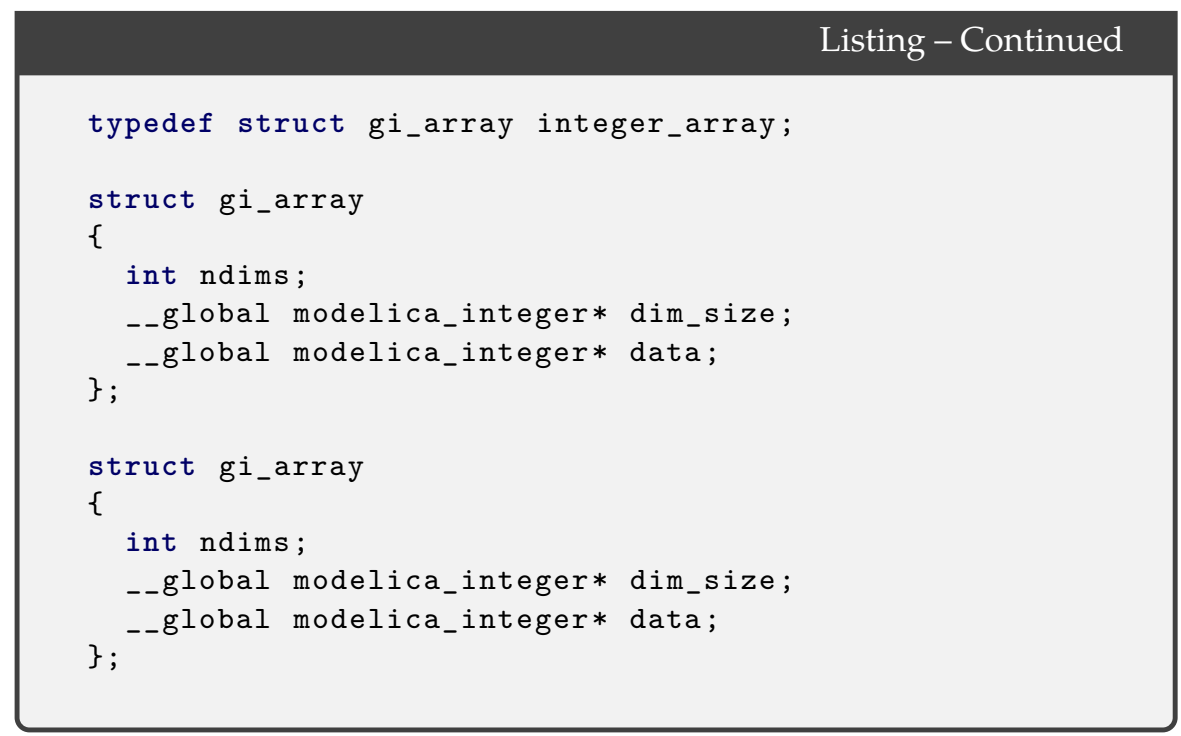

It defines the data structures used to represent parallel variables. These data structures are used to characterize parallel variables in the serial C code. The device integer array structure representing an integer array on a parallel device is shown in Listing 13.1.

The library is also responsible for all OpenCL related initialization operations like device selection, creating contexts on devices, building OpenCL source code from a source file or a string buffer, setting arguments to and launching kernels an so on. It provides clear and concise functions for OpenCL operations by hiding the rather long and complicated OpenCL operations in the background. It also provides all the mechanisms necessary for data transfer operations between the host and the OpenCL device. These include allocation and copy operations of: host to device, device to host and device to device.

Parallel implementations of some built-in functions (e.g. transpose()) are also available in the library. All the necessary mechanism for runtime error reporting related to OpenCL operations are also part of this library. Functions for debugging operations are also available in the library.

\subsection{ParModelica OpenCL Utility Headers}

Simulating a model using the OpenModelica compiler and runtime system involves $C$ code generation. The generated $C$ code is then compiled and linked with the libraries which provide operations for the simulation. For example the OpenModelica SimulationRuntimeC library (SimulationRuntimeC.a Win- 
dows or SimulationRuntimeC.so Linux) provides, among many other things, the structures and operations necessary to represent arrays.

With $\mathrm{C} / \mathrm{C}++$ it is possible to implement operations in one library and link them later if they are needed. OpenCL, on the other hand, has no linking mechanisms. This lack of linking mechanisms means that any utility methods needed by the implementation have to be in source code format and have to be compiled with the rest of the generated OpenCL code. the ParModelica utility headers provide this functionality. These headers provide the structures used for representing arrays, methods for copying and manipulating these arrays, a number of Modelica built-in functions, device specific configurations and so on. 



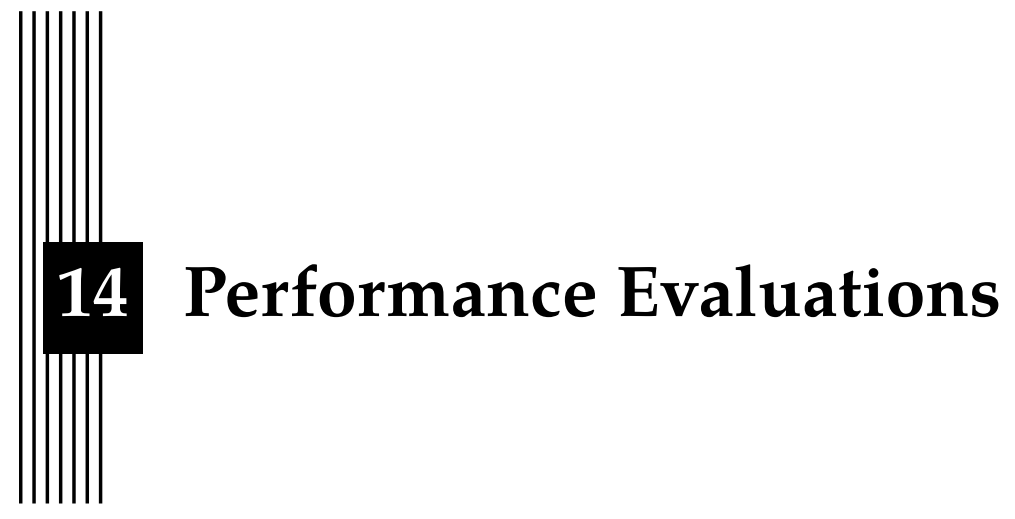

\subsection{The MPAR Benchmark Suite}

The MPAR benchmark test suite is a set of Modelica test models written using the new parallel extensions. It was developed as a separate Master's thesis work [51] to evaluate the performance of the ParModelica implementations.

The suite contains seven different algorithms from well-known benchmark applications such as the LINear equations software PACKage (LINPACK) [24], and Heat Conduction [71]. These benchmarks have been collected and implemented as algorithmic time-independent Modelica models. The algorithms implemented in this suite involve rather large computations and impose well defined work-loads on the OpenModelica compiler and the run-time system. Moreover, they include different kinds of for-loops and function calls which provide parallelism for domain and task decomposition.

Performance results for three out of the seven test models: Matrix Multiplication, Eigen value computation, and Stationary Heat Conduction, are presented in the next section. Time measurements have been performed for both sequential and parallel implementations of three models on both CPU and GPU architectures. For executing sequential codes generated by the standard sequential OpenModelica compiler an Intel Xeon E5520 CPU which has 16 cores, each with $2.27 \mathrm{GHz}$ clock frequency is used. For executing generated code by our the OpenCL based parallel code generator, we have used the same CPU as well as the NVIDIA Fermi-Tesla M2050 GPU with 448 cores. 


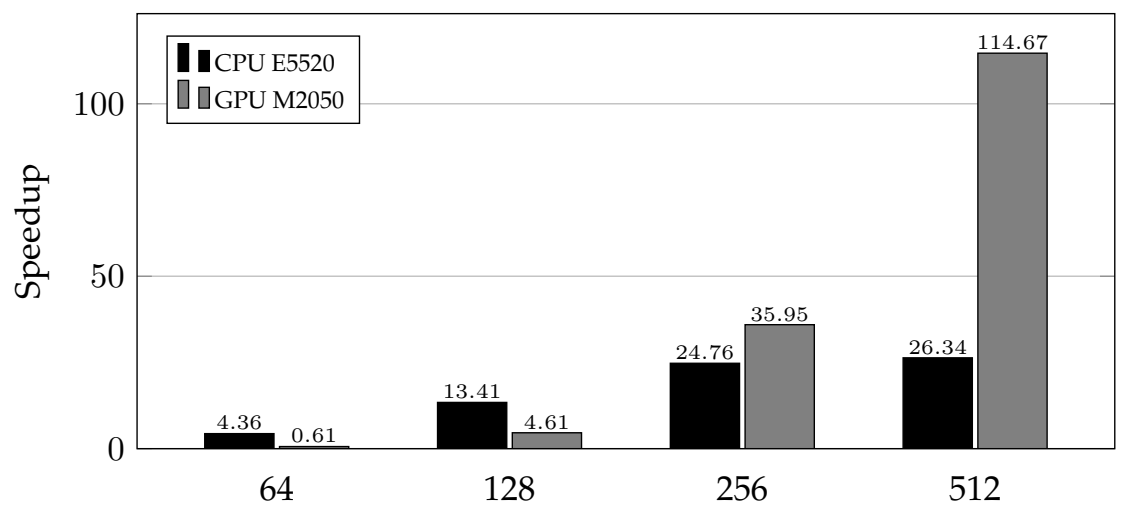

Figure 14.1: Speedups for matrix multiplication

\subsubsection{Results}

The first test case that is presented is the classic Matrix Multiplication computation. the function computes the result of multiplication of two square matrices of size $\mathrm{N}$ as shown in Equation (14.1).

$$
c_{i j}=\sum_{i=1}^{N} \sum_{j=1}^{N} \sum_{k=1}^{N} a_{i k} * b_{k j}
$$

Although rather straight forward, matrix multiplication is a good way to evaluate the performance of the new extensions and implementation. There are a number of ways to parallelize matrix multiplication with the available extensions. It can be implemented as a simple parfor loop, a parfor loop combined with parallel functions or a complete optimized parallelization using kernels and better thread and management features. This model presents a very large level of data-parallelism for which a considerable speedup has been achieved as a result of parallel simulation of this model on parallel platforms. Figure 14.1 shows the achieved speed-ups for the computation of different matrix sizes.

The second test model performs eigenvalue computation which is also a common linear algebra routine with a wide range of applications in many modeling areas. The test model computes all the eigenvalues of computation of a tridiagonal symmetric matrix. The observed speedups for this model are show in Figure 14.2.

The third test models specifies a stationary thermal conduction in a 2dimensional plate. The heat conduction problem computes the temperature distribution of a surface square plate $[0,1] \times[0,1]$ with given initial boundary 


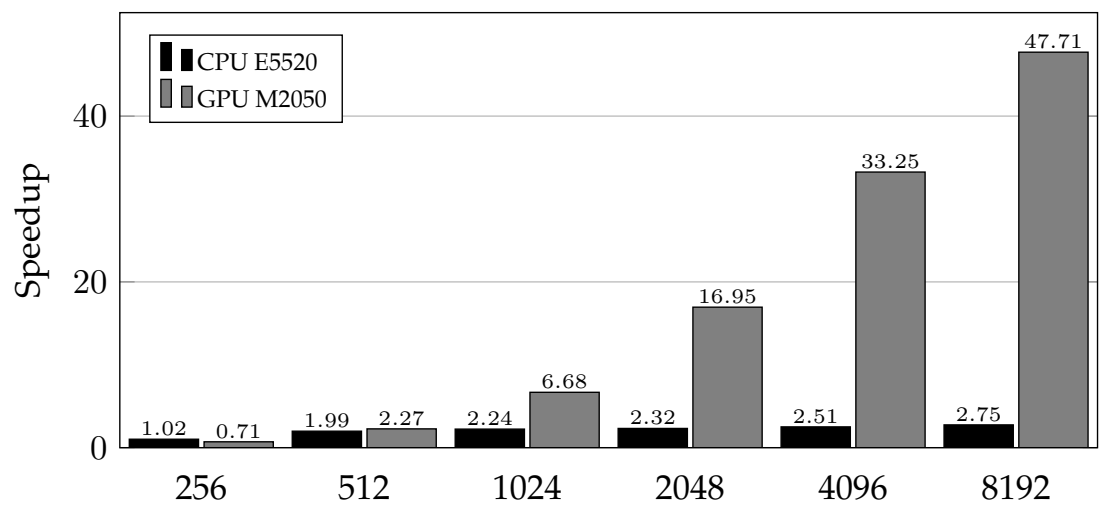

Figure 14.2: Speedups for Eigenvalue computations

\section{Figure 14.3: Equidistant computation grid}

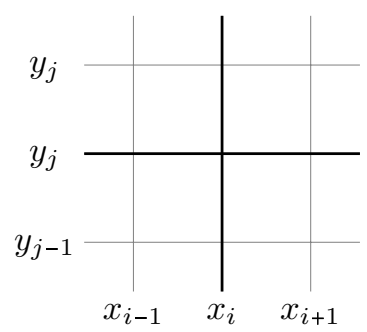

conditions / temperatures. The thermal distribution can be represented by the differential equation shown in Equation (14.2).

$$
\frac{\partial^{2} T}{\partial x^{2}}+\frac{\partial^{2} T}{\partial y^{2}}=0, \quad 0<x, y<1
$$

By defining an equidistant grid $\left(x_{i}, y_{i}\right)_{i, j=0}^{N+1}$ as shown in Figure 14.3 and using finite difference approximation methods [70] the heat distribution equation can be discretized as shown in Equation (14.3)

$$
-4 T_{i, j}+T_{i+1, j}+T_{i-1, j}+T_{i, j-1}+T_{i, j-1}=0
$$

where $T_{i, j}=T\left(x_{i}, y_{j}\right)$ is the approximated temperature at point $\left(x_{i}, y_{j}\right)$ in the discretized plate. 


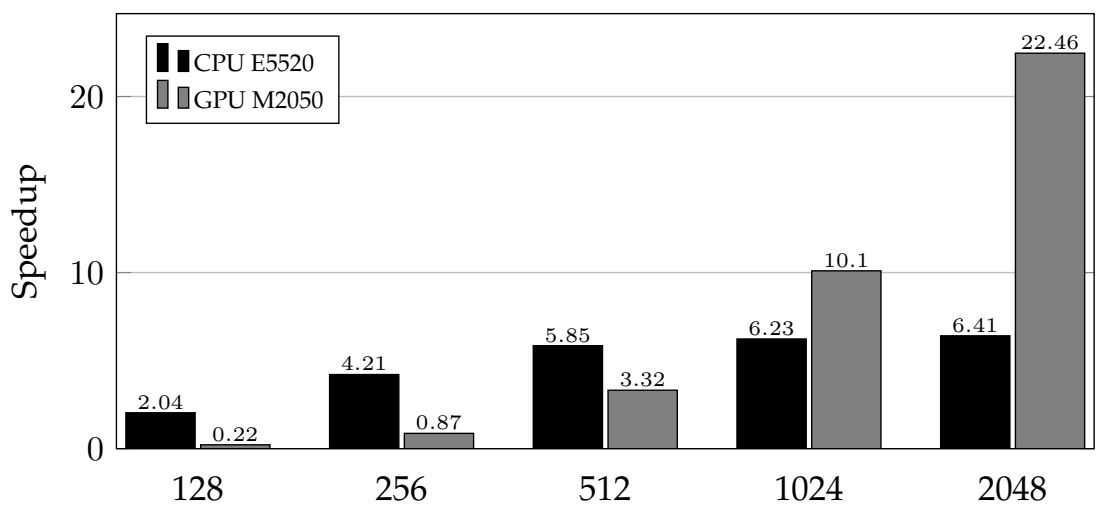

Figure 14.4: Speedups for 2d heat plate computations

Direct numerical methods such as Gaussian Elimination can be used to solve the system of linear equations. However, if the number of grid points is large, iterative methods such as Jacobi method are better suited. The iteration tries to approximate the next solution of the equation using the obtained solutions in previous iterations. If the approximate temperature $T_{i, j}^{k}$ is known for point $\left(x_{i}, y_{j}\right)$ at the $k_{t h}$ then the value at $T_{i, j}^{k+1}$ can be approximated as shown in Equation (14.4).

$$
T_{i, j}^{k+1}=\frac{T_{i+1, j}^{k}+T_{i-1, j}^{k}+T_{i, j-1}^{k}+T_{i, j-1}^{k}}{4} \quad 1 \leq i, j \leq N-1
$$

This computation is a bit more complicated to parallelize since iterations should be synchronized properly and data dependencies should be obeyed strictly. Specially since values from previous iterations are used to compute values for subsequent iterations.

The achieved speedups for the $2 \mathrm{~d}$ plate thermal conduction model are shown in Figure 14.4. 


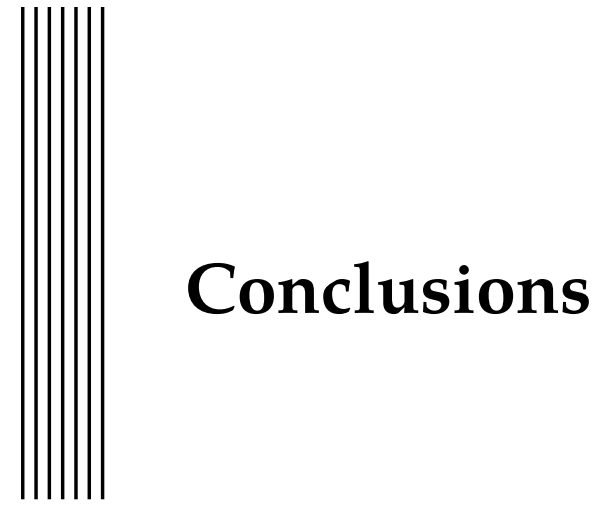

This thesis work attempts to reduce the prevalent gap between advances in modern parallel programming methods and modelling/simulation tools. The work investigated two main parallelization approaches: Automatic and Explicit parallelization. We formulated and analysed methods and techniques for mathematical simulations to be able to take advantage of the computational capabilities provided by modern multi-core CPUs and GPUs. The formalisms for each parallelization approach are augmented by the corresponding necessary implementations and assessed with evaluations to determine estimate the feasibility, usability, and performance of each approach.

The automatic parallelization direction, covered in Part I, investigated two separate approaches. The first approach, parallelization based on analysis of connected components discussed in Chapter 5, investigated low overhead, compile-time, static, and integrated methods for multi-threaded evaluations of equation systems with a high degree of decoupling. This is shown to be feasible in specific cases where modelling methods or symbolic manipulations by modelling tools result in systems of equations with multiple disconnected subsystems of equations. This approach can also benefit from specific modelling techniques that can formulate physical systems in ways that improve decouplings.

The second automatic parallelization approach, covered in Chapters 6 to 9 tries to address and improve the analysis, design, and implementation issues encountered by the first approach. The main target in this approach is to design and provide a widely applicable, adaptive, flexible and portable parallelization scheme. We investigate an approach based on analysis of strongly 
connected components of large and complex equation system with heavy dependencies. Multiple clustering heuristics and scheduling methods are investigated and implemented. These heuristics and methods are shown to be effective in processing and executing large systems of complicated equation systems resulting from models implemented in equation-based modelling languages such as Modelica. The approach is based on runtime processing which allows for a more responsive design that can adjust to the dynamic behaviour of task systems with adaptive runtime scheduling and rescheduling capabilities.

Most of the processing and functionality needed for this parallelization approach is implemented in the ParModAuto task system library. The implementation is designed to be sufficiently high level, flexible, and portable. This library-based design opens up the opportunity for other tools to adapt and incorporate automatic parallelization with relative ease. The performance of this approach and the corresponding implementation has been investigated within the OpenModelica Modeling and Simulation Environment. Evaluations are performed with models from multiple domains such as the Electrical, Mechanical and Thermal areas. The test cases demonstrate a considerable amount of performance improvement. Furthermore, the benefit of parallelization in this approach is going to get better as models get larger.

The second part of the thesis, Part II, investigates the feasibility of explicit parallel programming in high level modelling and simulation languages, specifically in Modelica. We introduced the ParModelica Extensions to the Modelica language to allow for an explicit parallel programming approach inspired by modern GPGPU frameworks/languages. This provided, for the first time, an integrated, seamless and clean way to design parallel programs in the Modelica language. The new extensions together with the high level nature of Modelica open up the possibility to design parallelized algorithms directly in Modelica. This is a much needed feature considering the fact that most modellers who use the language are not familiar with low level languages which are, usually, the choice for parallel programming. The explicit programming extensions are demonstrated in a selected number of small applications and show large speedups on suitable algorithms with large data-parallel computational problems. 


\section{Part III}

Appendix 



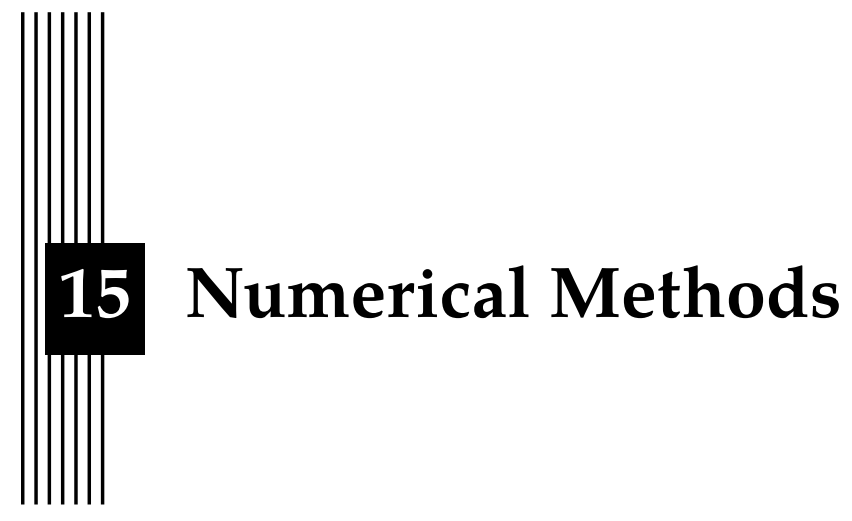

Mathematical simulation and modelling is inherently dependent on a good deal of Linear Algebra, Differential and Numerical Methods. These include, but are not limited to, methods for solving Linear and Non-Linear Equation solvers, event handling methods, numerical differentiation and integration methods for Ordinary and Algebraic Differential Equation. Modelling languages and tools need to utilize a number of these methods if they are to handle the wide variety of physical problems nature puts forward.

The next sections will try to give a brief overview of some of these methods. Of course, this is by no means a complete discussion. It is only meant to be quick reference for a few selected numerical methods to help with the discussion is the main part of the document.

\subsection{Non-Linear Systems: Root Finding and Newton's method}

Newton's method also known as Newton-Raphson method is one of the most popular methods for solving a system of non-linear equations. Given a function $f: \mathbb{R} \rightarrow \mathbb{R}$ and an initial guess for $x=x^{0}$, Newton's method tries to find the root of the function, i.e., find

$$
x: f(x)=0
$$


with a fixed point iteration scheme

$$
x^{k+1}=x^{k}-\frac{f\left(x^{k}\right)}{f^{\prime}\left(x^{k}\right)}
$$

If started with a good enough initial guess $x^{0}$, Newton's method usually very efficient in finding the solution. So the intial guess should be chosen systematically and with care.

Newton's method can be used not just for single functions but also for a system of multi-variable functions or equation systems. For a system of, possibly non-linear, multi-variable functions $\mathcal{F}: \mathbb{R}^{n} \rightarrow \mathbb{R}^{n}$

$$
\mathcal{F}(X)=\left[\begin{array}{c}
f_{1}(X) \\
f_{2}(X) \\
\vdots \\
f_{n}(X)
\end{array}\right]= \begin{cases}f_{1}\left(x_{1}, x_{2}, \ldots, x_{n}\right) & =0 \\
f_{2}\left(x_{1}, x_{2}, \ldots, x_{n}\right) & =0 \\
\vdots & \\
f_{n}\left(x_{1}, x_{2}, \ldots, x_{n}\right) & =0\end{cases}
$$

where $\mathcal{F}$ is a column vector of functions, $X$ is a column vector of variables, Newton's method's approximation scheme of Equation (15.2) can be generalized as

$$
X^{k+1}=X^{k}-\frac{\mathcal{F}\left(X^{k}\right)}{\mathcal{J}\left(X^{k}\right)}
$$

where $\mathcal{J}$ is the Jacobian matrix defined over the function vector $\mathcal{F}$ as:

$$
\mathcal{J}=\left[\begin{array}{cccc}
\frac{\partial f_{1}}{\partial x_{1}} & \frac{\partial f_{1}}{\partial x_{2}} & \cdots & \frac{\partial f_{1}}{\partial x_{n}} \\
\frac{\partial f_{2}}{\partial x_{1}} & \frac{\partial f_{2}}{\partial x_{2}} & \cdots & \frac{\partial f_{2}}{\partial x_{n}} \\
\vdots & \vdots & \ddots & \vdots \\
\frac{\partial f_{n}}{\partial x_{1}} & \frac{\partial f_{n}}{\partial x_{2}} & \cdots & \frac{\partial f_{n}}{\partial x_{n}}
\end{array}\right]
$$

with the $i j$ th element being $\frac{\partial f_{i}}{\partial x_{j}}$. We can now solve for the next approximation of $X$ as

$$
X^{k+1}=X^{k}-\mathcal{J}^{-1}\left(X^{k}\right) \mathcal{F}\left(X^{k}\right)
$$

where $\mathcal{J}^{-1}$ is the inverse of the Jacobian matrix. However, inverting a matrix is a very expensive operation and is not usually done directly in practice, even for relatively small matrix sizes. Instead, we can write Equation (15.4) as the linear system

$$
\begin{aligned}
\mathcal{J}\left(X^{k}\right)\left(X^{k+1}-X^{k}\right) & =-\mathcal{F}\left(X^{k}\right) \\
\mathcal{J}\left(X^{k}\right) s^{k} & =-\mathcal{F}\left(X^{k}\right)
\end{aligned}
$$

We can now solve for $\mathbf{s}^{k}$ in every iteration using a linear system method, such as Gaussian Elimination, and compute the next approximate solution, $X^{k+1}$ as 


$$
X^{k+1}=X^{k}+s^{k}
$$

Newton's Method and its variants are some of the most popular and widely used methods for solving systems of non-linear equations. In practice, with an educated initial guess, they usually have satisfactory convergence and stability. Modelling and Simulation tools such as OpenModelica use these methods to handle non-linear systems of equations composed of hundreds of equations efficiently.

\subsection{Numerical Integration}

A decent mathematical modelling language and tool is expected to support systems including differential equations such as the form shown in Equation (3.24) since rates of changes, i.e. derivatives, are a vital part of our perception of the world. The relationship between variables and their derivatives allows us to compute one from the other using the Fundamental Theorems of Calculus. Numerical integration methods essentially allow the computation of a function $f(x)$ given its derivative $f^{\prime}(x)$. That is compute an approximate solution to a definite integral:

$$
f(x)=\int_{a}^{b} f^{\prime}(x) d x
$$

Suppose we have a derivative $\dot{x}$ of the variable $x$ defined by a function/equation over an independent variable $t$ and an initial value $x_{0}$ for that variable at $t_{0}$, that is:

$$
\dot{x}=f(t), \quad x_{0}=\left.x\right|_{t_{0}}
$$

The value of $x$ at a finite interval $t_{1}=t_{0}+\delta t$ can be computed as

$$
x_{1}=x_{0}+\int_{t_{0}}^{t_{1}} f(t) d t
$$

This can be generalized for points $x_{n}, t_{n}$ and $t_{n+1}$ as

$$
x_{n+1}=x_{n}+\int_{t_{n}}^{t_{n+1}} f(t) d t
$$

Numerical Integration methods can be used compute the integral on the right hand side of Equation (15.12) and approximate the solution trajectory of $x$ by successive evaluations.

There exist wide variety of numerical integration algorithms developed over the years. Some are general purpose methods while others target specific types of problems. A detailed and advanced analysis of these methods can be found in [90] [67] and a good deal of other literature. Some of the most widely used class of numerical integrations methods are based on Polynomial Interpolations that approximate the solution trajectories. 


\section{Figure 15.1: Euler's Methods}

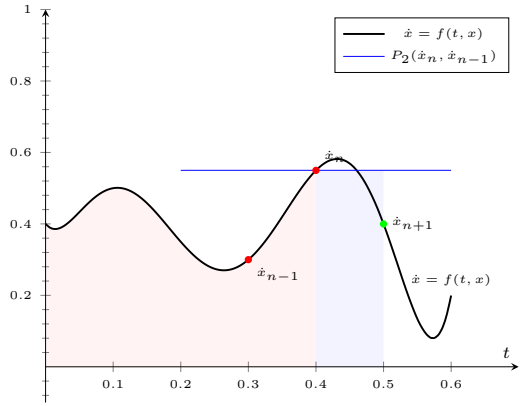

(a) Forward Euler

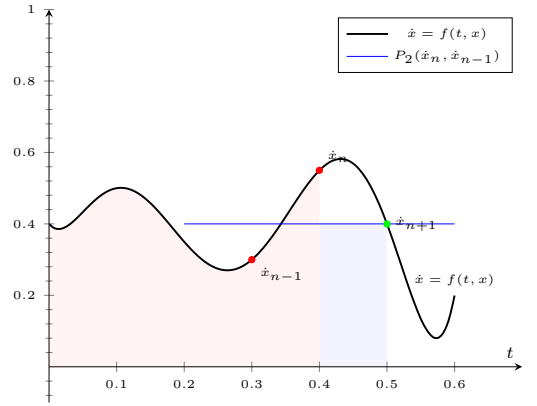

(a) Backward Euler

\subsubsection{Euler's Methods}

Euler's Methods are perhaps the simplest Numerical Integration methods. These methods use a 0 degree polynomial, i.e., a constant, to approximate $\dot{x}$ around a given point. There are two forms of this method depending on which time point is used for the polynomial. The first method is the Forward Euler's Method (or just Euler's Method) illustrated in Figure 15.2a. Here $\dot{x}_{n}$, the value of $\dot{x}$ at time step $t_{n}$, is used as the 0 degree polynomial (a constant) ${ }^{1} P_{0}$ to approximate $\dot{x}$ around $t_{n}$. That is

$$
P_{0}(t)=\dot{x}_{n}
$$

Integrating this polynomial from $t_{n}$ to $t_{n+1}$

$$
\begin{aligned}
\int_{t_{n}}^{t_{n+1}} P_{0}(t) d t & =\left(t_{n+1}-t_{n}\right) \cdot \dot{x}_{n} \\
& =h \cdot \dot{x}_{n} \\
& =h \cdot f\left(t_{n}, x_{n}\right)
\end{aligned}
$$

now we can substitute Equation (15.14) into Equation (15.12) and solve for $x_{n+1}$

$$
x_{n+1}=x_{n}+h \cdot f\left(t_{n}, x_{n}\right)
$$

Which is the Forward Euler's Method. In much simpler terms The Forward Euler's Method calculates the are area under the curve $\dot{x}=f(x, t)$ between $t_{n}$ and $t_{n+1}$ using the rectangle of height $\dot{x}_{n}$ as illustrated by the blue shaded area in Figure 15.2a. The area shaded red is $x_{n}$.

\footnotetext{
${ }^{1}$ Technically the degree of the zero polynomial $\mathrm{P}(\mathrm{t})=0$ is left undefined. Here, however, it is of no consequence for the discussion so it is treated as degree 0 polynomial.
} 


\section{Figure 15.4: $3^{\text {rd }}$ order Adam-Bashforth}

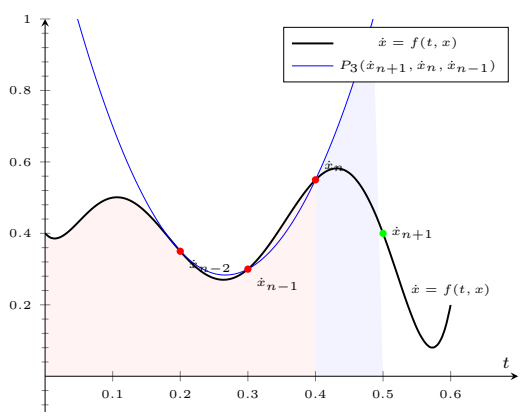

(a)

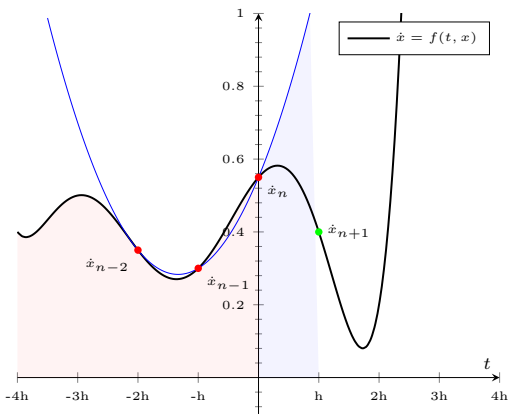

(b)

Similarly, using $P_{0}(t)=\dot{x}_{n+1}$ as shown in Figure 15.3a, the Backward Euler's Method can be derived as

$$
x_{n+1}=x_{n}+h \cdot f\left(t_{n+1}, x_{n+1}\right)
$$

This is an implicit method. It is an implicit method since $x_{n+1}$, the value we are trying to compute, is used in the right hand side of the equation. One way to solve such an implicit formulation for $x_{n+1}$ would be to employ NewtonRaphson's root finding explained in Section 15.1.

Perhaps it is already evident that Euler's methods are inadequate methods in terms of accuracy. Both methods basically assume the derivative, $\dot{x}$, is constant for the whole time-step. In other words these two methods disregard any behaviour of the system around the current time instant and only consider the instantaneous value. While they are simple and very easy to implement they are often either highly inaccurate or inefficient. One possible way to improve the accuracy of these methods is to use perpetually smaller time-steps. However, this will lead to a very inefficient method in terms of computational time. Another approach is to make use of more previously solved $\dot{x}$ or $x$ values to obtain a better approximating polynomial. Such methods are called Multi-Step Methods.

\subsubsection{Adam-Bashforth Methods}

These methods are a family of multi-step explicit methods. The AdamBashforth family of methods use previous values of the derivative $\dot{x}_{n}, \dot{x}_{n-1}, \dot{x}_{n-2} \ldots$ to interpolate a polynomial approximation of $\dot{x}=f(x, t)$. Then they use these polynomial to extrapolate the value of $\dot{x}_{n+1}$.

Consider the graph of a certain $\dot{x}=f(x, t)$ in Figure 15.5a. Here we can use the three points $\left\{\dot{x}_{n-2}, \dot{x}_{n-1}, \dot{x}_{n}\right\}$ to get an approximating polynomial for 
$f(x, t)$ around these points. To make the amount of algebra we have to deal with, we can instead imagine the y-axis being shifted to align with $\dot{x}_{n}$ as shown in Figure $15.5 \mathrm{~b}^{2}$. In addition, note that $t_{n+1}-t_{n}=h$ for any $n$, i.e., we have a fixed step size.

The quadratic polynomial interpolating the three points $\left\{\dot{x}_{n-2}, \dot{x}_{n-1}, \dot{x}_{n}\right\}$ in Lagrange form can now be written as

$$
P_{2}(t)=\frac{(t+2 h)(t+h)}{2 h^{2}} \cdot \dot{x}_{n}-\frac{t(t+2 h)}{h^{2}} \cdot \dot{x}_{n-1}+\frac{t(t-h)}{2 h^{2}} \cdot \dot{x}_{n-2}
$$

The area under the curve of this approximating polynomial between the points $\left\{\dot{x}_{n}, \dot{x}_{n+1}\right\}$ is the definite integral between 0 and $h^{3}$, i.e.,

$$
\int_{0}^{h} P_{2}(t) d t=\frac{h}{12}\left(23 \dot{x}_{n}-16 \dot{x}_{n-1}+5 \dot{x}_{n-2}\right)
$$

We can now substitute Equation (15.18) into Equation (15.12) and simplifying accordingly to get an approximation of $x_{n+1}$ as

$$
\begin{aligned}
x_{n+1} & =x_{n}+\frac{h}{12}\left(23 \dot{x}_{n}-16 \dot{x}_{n-1}+5 \dot{x}_{n-2}\right) \\
& =x_{n}+\frac{h}{12}\left(23 f\left(t_{n}, x_{n}\right)-16 f\left(t_{n-1}, x_{n-1}\right)+5 f\left(t_{n-2}, x_{n-2}\right)\right)
\end{aligned}
$$

This is the explicit third order Adam-Bashforth Linear Multi-Step Method. these are explicit methods since the next approximation $x_{n+1}$ is computed using only previously computed values of $\dot{x}$. Higher degree Adam-Bashforth Methods can be formulated in a similar manner using more points to compute the approximating polynomial function.

\subsubsection{Adam-Moulton Methods}

The Adam-Bashforth family of methods discussed in the previous section use previous values of the derivative $\dot{x}_{n}, \dot{x}_{n-1}, \dot{x}_{n-2} \ldots$ to interpolate a polynomial approximation of $\dot{x}=f(x, t)$. Then they use these polynomial to extrapolate the value of $\dot{x}_{n+1}$. Adam-Moulton methods are quite similar except they approximate $\dot{x}_{n+1}$ using interpolation instead of extrapolation. That means the point $\dot{x}_{n+1}$ is used in computing the approximating polynomial.

Consider the graph of a certain $\dot{x}=f(x, t)$ in Section 15.2.3. Here we can use the three points $\left\{\dot{x}_{n-1}, \dot{x}_{n}, \dot{x}_{n+1}\right\}$ to get an approximating polynomial

\footnotetext{
${ }^{2}$ Equivalent to replacing $t$ with $t=m+4 h$ and then replacing $m$ by $t-4 h$ at the end. It should not make any difference in the result because $t$ does not appear in the final result as we will see.

${ }^{3}$ solve
} 


\section{Figure 15.6: $3^{\text {rd }}$ order Adam-Moulton}

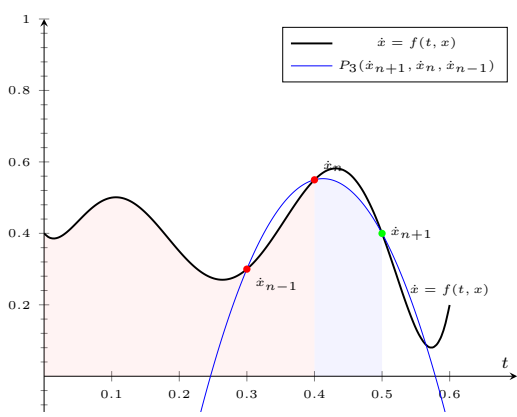

(a)

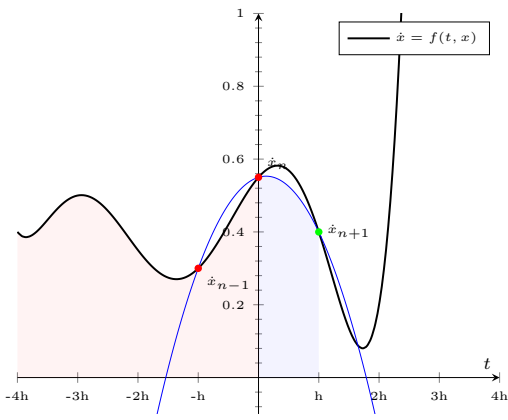

(b)

for $f(x, t)$ around these points. Using the same approach used for AdamBashforth methods above, we can imagine the y-axis being shifted to align with $\dot{x}_{n}$ as shown in Figure 15.7b. In addition, note that $t_{n+1}-t_{n}=h$ for any $n$, i.e., we have a fixed step size.

The quadratic polynomial interpolating the three points $\left\{\dot{x}_{n-1}, \dot{x}_{n}, \dot{x}_{n+1}\right\}$ in Lagrange form can now be written as

$$
P_{2}(t)=\frac{t(t+h)}{2 h^{2}} \cdot \dot{x}_{n+1}-\frac{(t-h)(t+h)}{h^{2}} \cdot \dot{x}_{n}+\frac{(t-h) t}{2 h^{2}} \cdot \dot{x}_{n-1}
$$

The area under this curve between the points $\left\{\dot{x}_{n}, \dot{x}_{n+1}\right\}$ is the definite integral of this polynomial between 0 and $h^{4}$, i.e.,

$$
\begin{aligned}
\int_{0}^{h} P_{2}(t) d t & =\frac{h}{12}\left(5 \dot{x}_{n+1}+8 \dot{x}_{n}-\dot{x}_{n-1}\right) \\
& =\frac{h}{12}\left(5 f\left(t_{n+1}, x_{n+1}\right)+8 f\left(t_{n}, x_{n}\right)-f\left(t_{n-1}, x_{n-1}\right)\right)
\end{aligned}
$$

We can now substitute Equation (15.21) into Equation (15.12) and simplifying accordingly to get an approximation of $x_{n+1}$ as

$$
x_{n+1}=x_{n}+\frac{h}{12}\left(5 f\left(t_{n+1}, x_{n+1}\right)+8 f\left(t_{n}, x_{n}\right)-f\left(t_{n-1}, x_{n-1}\right)\right)
$$

This is the third order Adam-Moulton Linear Multi-Step Method. Note that these methods are implicit methods since the value we are trying to approximate, $x_{n+1}$, is involved in the right hand side of the formulation. These

${ }^{4}$ solve 


\section{Figure 15.8: $2^{\text {nd }}$ order Backward Differentiation Formulae}

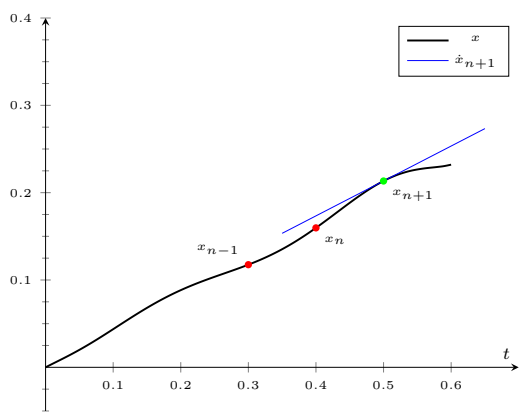

(a)

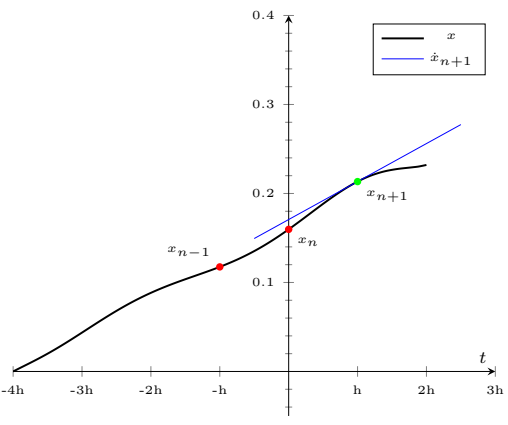

(b)

methods require non-linear solvers such as Newton's method to solve this implicit formulation on every evaluation. Higher degree Adam-Moulton Methods can be formulated in a similar manner using more points to compute the approximating polynomial function.

\subsubsection{Backward Differentiation Formulae (BDF) Methods}

The Adam-Moulton and Adam-Bashforth family of methods were based on interpolating the polynomial approximation of $\dot{x}$ and using this polynomial to compute the approximated integral $x$. The Backward Differentiation Formulae family of methods, on the other hand, use a polynomial approximation of $x$. We can then differentiate this polynomial to get an approximation of the derivative $\dot{x}$.

Consider the system shown in Figure 15.9a. If we want to approximate $\mathrm{x}$ by the polynomial interpolating the three points $\left\{x_{n-1}, x_{n}, x_{n+1}\right\}$, we can use the same approach we used above and shift the axis to line up with $x_{n}$ as shown in Figure 15.9b. Then the polynomial through these three points in Lagrange form can be written as

$$
x=P_{2}(t)=\frac{t(t+h)}{2 h^{2}} \cdot x_{n+1}-\frac{(t-h)(t+h)}{h^{2}} \cdot x_{n}+\frac{(t-h) t}{2 h^{2}} \cdot x_{n-1}
$$

Differentiating this polynomial gives

$$
\dot{x}=f(x, t)=\frac{d P_{2}}{d t}(t)=\frac{x_{n+1} \cdot(h+2 t)-x_{n-1} \cdot(h-2 t)-4 x_{n} \cdot t}{2 h^{2}}
$$


Evaluating Equation (15.24) at $t_{n+1}$, i.e., $t=h$ gives

$$
\begin{aligned}
\dot{x}_{n+1} & =\frac{x_{n+1} \cdot(h+2 h)-x_{n-1} \cdot(h-2 h)-4 x_{n} \cdot h}{2 h^{2}} \\
& =\frac{3 x_{n+1} h+x_{n-1} h-4 x_{n} h}{2 h^{2}}
\end{aligned}
$$

Simplifying and solving for $x_{n+1}$

$$
\begin{aligned}
x_{n+1} & =\frac{1}{3}\left(4 x_{n}-x_{n-1}+2 h \cdot \dot{x}_{n+1}\right) \\
& =\frac{1}{3}\left(4 x_{n}-x_{n-1}+2 h \cdot f\left(x_{n+1}, t_{n+1}\right)\right)
\end{aligned}
$$

This is the implicit BDF2 method. It should be noted that the BDF methods use previous values of $x$ as opposed to the Adam-Moulton and AdamBashforth methods which use previous $\dot{x}$ values to approximate the solution.

\subsubsection{DASSL}

BDF methods are some of the most widely used implicit multi-step methods in practice. The default solver in the OpenMoldeica (and also Dymola) simulation environment is called DASSL. DASSL [88] is a variable-order, variablestep BDF solver. It uses adaptive implicit BDF methods of order one to five to solve a given differential algebraic system. The step size and order of the BDF method at each step is chosen depending on the behaviour of the system around that time step. DASSL (or in general any 5th order implicit multistep method) can be relatively inefficient when solving non-stiff systems compared to other lower order multi-step methods. However, large and complex DAE systems with many equations are usually stiff. Considering that it can also adapt the order of the BDF method and the step size used depending on the problem, plus the fact that the implementation (the interface and the code) has been quite robust in practical usage, DASSL (and its successor SUNDIALS IDA [52]) have become the default choice of solver in many modelling and simulation environments. 



\section{$\|$ |||||| ParModelica (Extended Modelica) Concrete Syntax}

\subsection{Lexical conventions}

The following syntactic meta symbols are used (extended BNF):

[ ] optional

\{\} repeat zero or more times

I or

"text" The text is treated as a single token (no whitespace between any characters)

The following lexical units are defined (the ones in boldface are the ones used in the grammar, the rest are just internal to the definition of other lexical units):

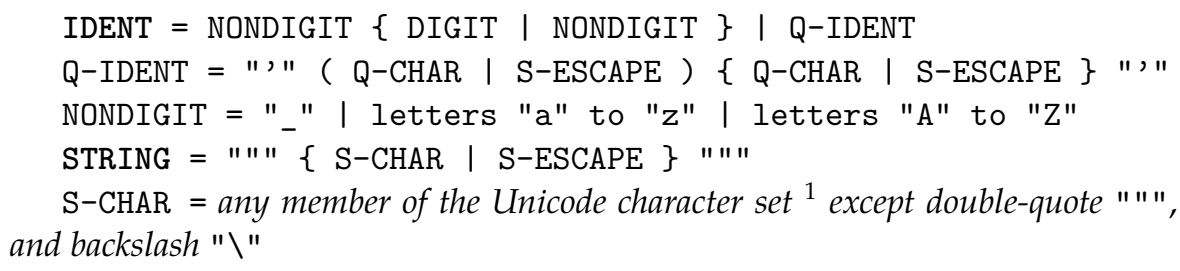
and backslash "\"

\footnotetext{
${ }^{1}$ http://www.unicode.org
} 


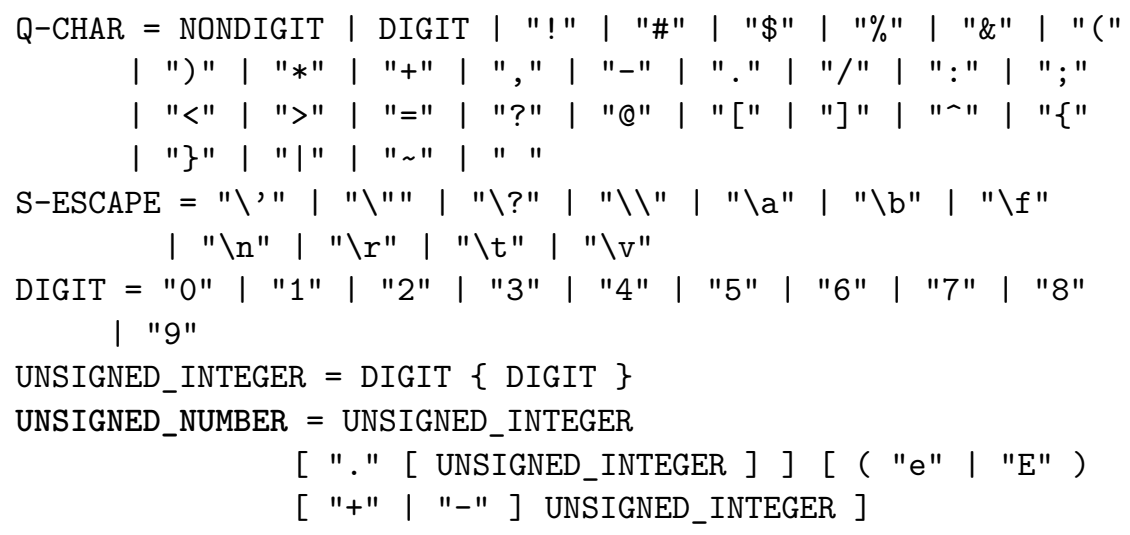

[The single quotes are part of an identifier. E.g. ' $x$ ' and $x$ are different IDENTs].

Note:

- Whitespace and comments can be used between separate lexical units and/or symbols, and also separates them. Whitespace and comments cannot be used inside other lexical units, except for STRING and QIDENT where they are treated as part of the STRING or Q-IDENT lexical unit.

- String constant concatenation "a" "b" becoming "ab" (as in C) is replaced by the "+" operator in Modelica.

- Modelica uses the same comment syntax as $\mathrm{C}++$ and Java (i.e., // signals the start of a line comment and $/ * \ldots *$ / is a multi-line comment); comments may contain any Unicode character. Modelica also has structured comments in the form of annotations and string comments.

- Description strings (= production "string_comment" in the grammar) and strings in annotations (= STRING with production annotation in the grammar) may contain any member of the Unicode character set. All other strings have to contain only the sub-set of Unicode characters identical with the 7-bit US-ASCII character set. [As a consequence, operators like " $>$ " or "<", and external functions only operate on ASCII strings and not on Unicode-strings. Within a description string the tags $<H T M L>$ and $</ H T M L>$ or $<h t m l>$ and $</ h t m l>$ define optionally begin and end of content that is HTML encoded.]

- Boldface denotes keywords of the Modelica language. Keywords are reserved words and may not be used as identifiers. 


\subsection{Grammar}

\subsubsection{Stored Definition - Within}

$$
\begin{aligned}
\langle\text { stored_definition }\rangle \quad::= & {[\text { 'within' [ 'name' }] \text { '; ' }] } \\
& \{[\text { 'final' }]\langle\text { class_definition }\rangle \text { '; }\}
\end{aligned}
$$

\subsubsection{Class Definition}

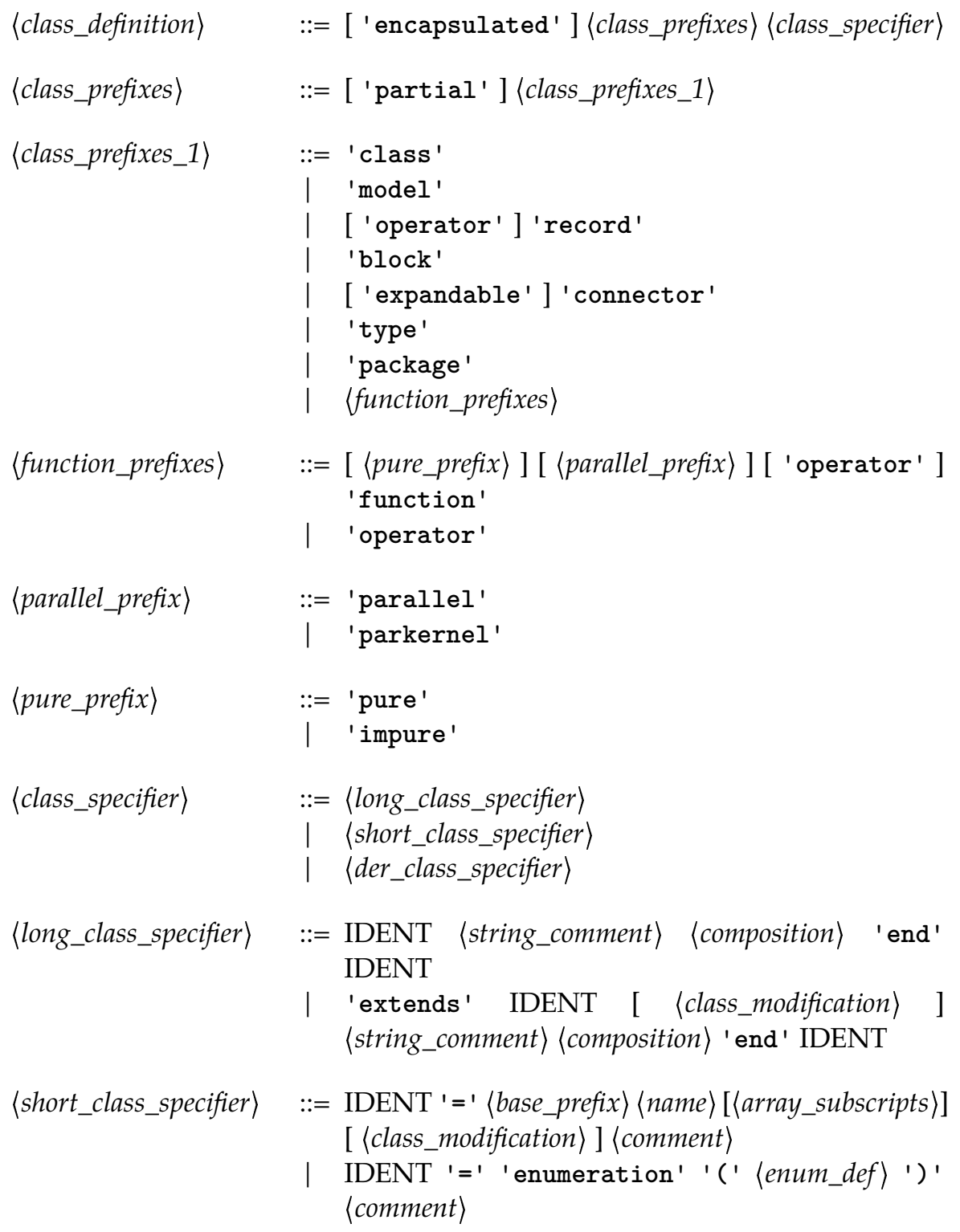




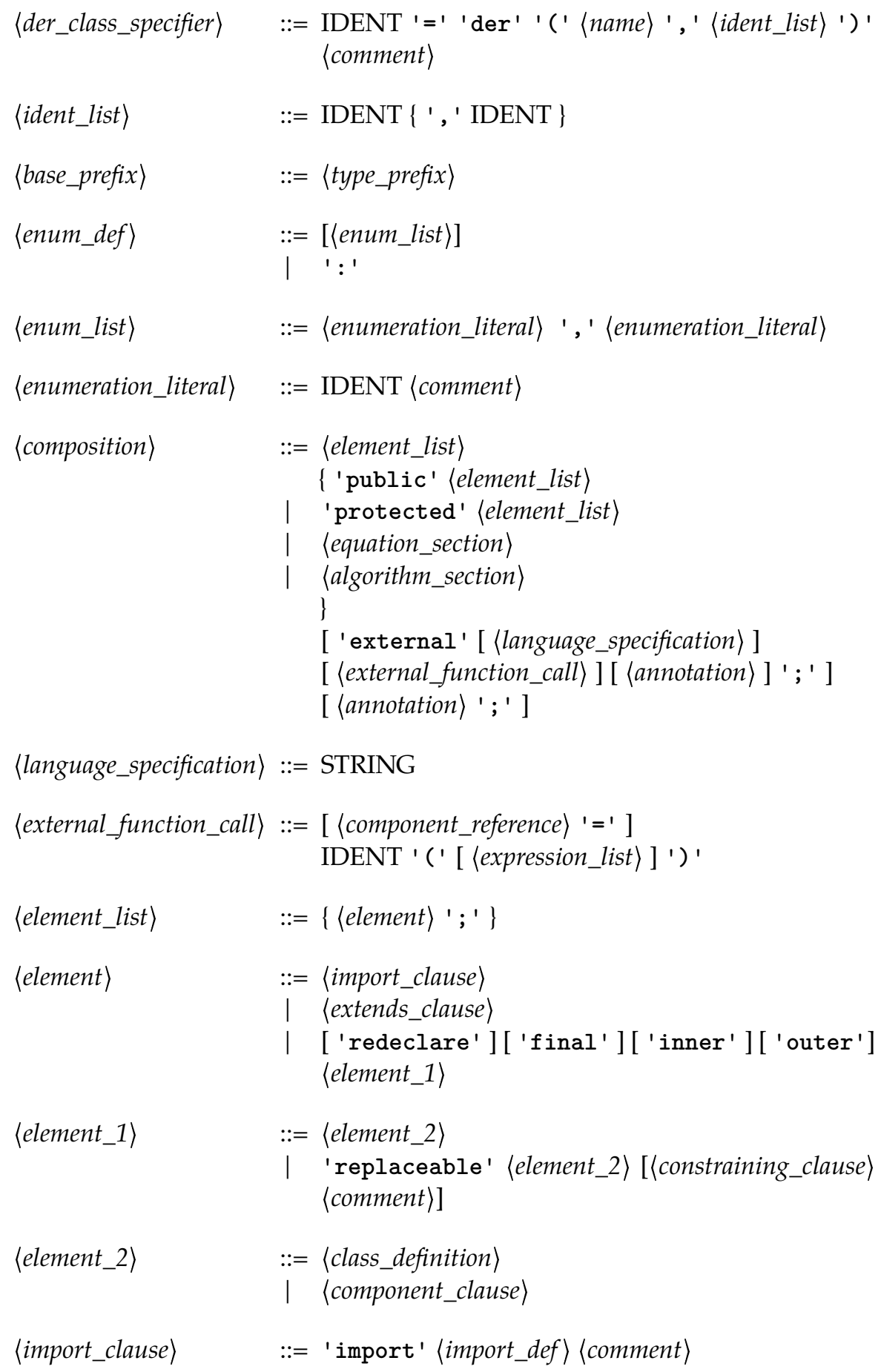




\begin{tabular}{|c|c|}
\hline$\langle$ import_def $\rangle$ & $\begin{array}{l}::=\text { IDENT }{ }^{\prime}=\text { ' }\langle\text { name }\rangle \\
\mid \quad\langle\text { name }\rangle\left[{ }^{\prime} . '\langle\text { import_def_1 }\rangle\right]\end{array}$ \\
\hline$\langle$ import_def_1 $\rangle$ & $::=' * 1$ \\
\hline
\end{tabular}

\subsubsection{Extends}

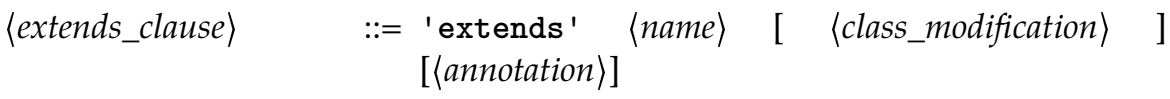

$\langle$ constraining_clause $\rangle \quad::=$ 'constrainedby' $\langle$ name $\rangle[\langle$ class_modification $\rangle]$

\subsubsection{Component Clause}

\begin{tabular}{|c|c|}
\hline$\langle$ component_clause $\rangle$ & $\begin{aligned}::= & \langle\text { type_prefix }\rangle\langle\text { type_specifier }\rangle[\langle\text { array_subscripts }\rangle \\
& \langle\text { component_list }\rangle\end{aligned}$ \\
\hline$\langle$ type_prefix $\rangle$ & $\begin{aligned}::= & {[\text { 'flow' | 'stream' ] }} \\
& \text { [ 'discrete' | 'parameter' | 'constant'] } \\
& \text { [ 'input' | 'output' ] } \\
& \text { [ 'parlocal' | 'parglobal'] }\end{aligned}$ \\
\hline$\langle$ type_specifier $\rangle$ & $::=\langle$ name $\rangle$ \\
\hline$\langle$ component_list $\rangle$ & $::=\langle$ component_decl $\rangle\left\{{ }^{\prime}, '\langle\right.$ component_decl $\left.\rangle\right\}$ \\
\hline component_decl $\rangle$ & $::=\langle$ declaration $\rangle[\langle$ condition_attribute $\rangle]\langle$ comment $\rangle$ \\
\hline$\langle$ condition_attribute $\rangle$ & $::=$ 'if' $\langle$ expression $\rangle$ \\
\hline claration $\rangle$ & $::=$ IDENT [ $\langle$ array_subscripts $\rangle][\langle$ modification $\rangle]$ \\
\hline
\end{tabular}

\subsubsection{Modification}

(modification

$::=\langle$ class_modification $\rangle\left[\mathrm{I}^{\prime}=\mathrm{I}^{\prime}\langle\right.$ expression $\left.\rangle\right]$

$\mid \quad '=1$ 'expression $\rangle$

$\mid \quad ':={ }^{\prime}\langle$ expression $\rangle$

$\langle$ class_modification $\rangle \quad::={ }^{\prime}\left({ }^{\prime}[\langle\text { argument_list }\rangle] \text { ' }\right)^{\prime}$

$\langle$ argument_list $\rangle \quad::=\langle$ argument $\rangle\left\{{ }^{\prime},{ }^{\prime}\langle\right.$ argument $\left.\rangle\right\}$

$\langle$ argument $\rangle \quad::=\langle$ element_mod_or_repl $\rangle$

| 〈element_redeclaration>

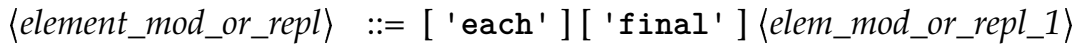




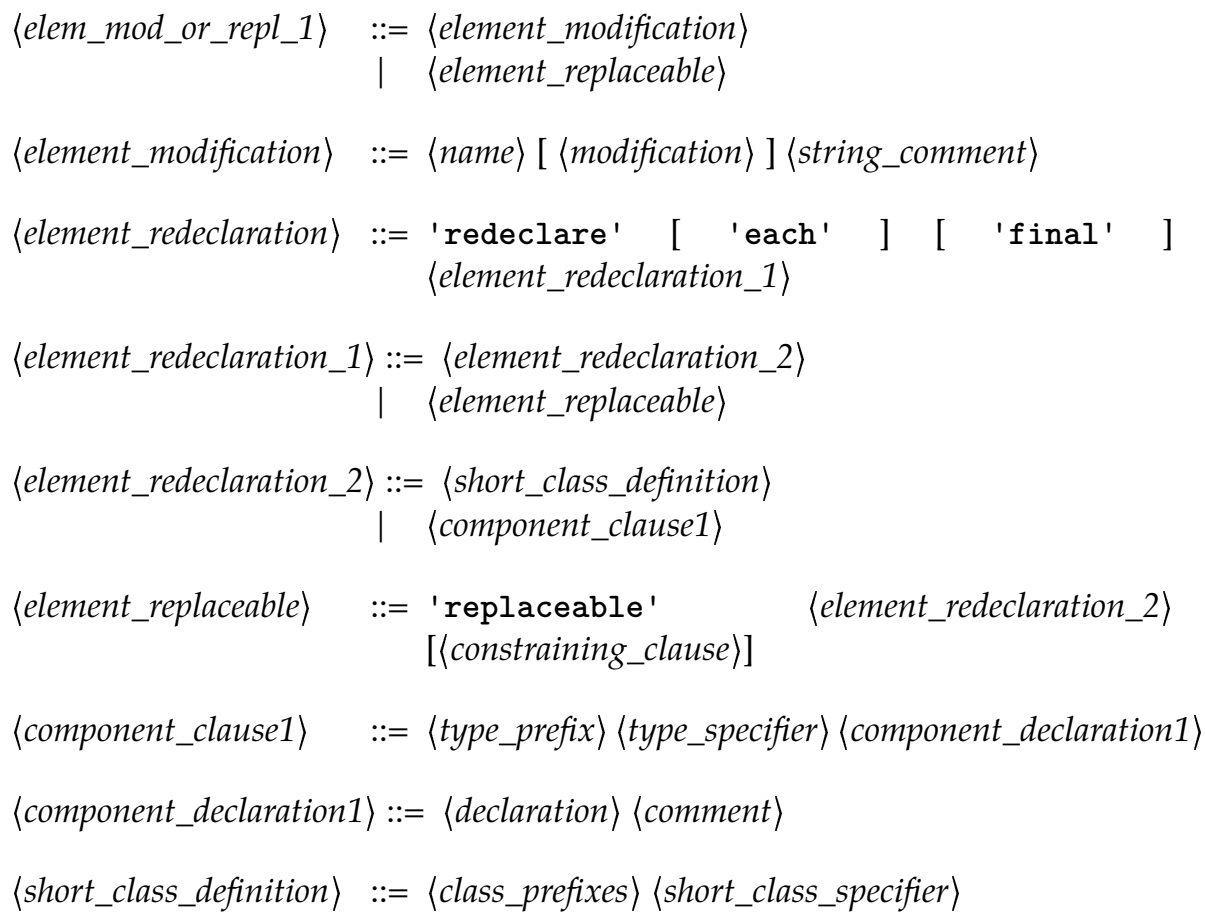

\subsubsection{Equations}

\begin{tabular}{|c|c|}
\hline$\langle$ equation_section $\rangle$ & $::=$ 'initial' 'equation' 〈equation_list $\rangle$ \\
\hline$\langle$ algorithm_section $\rangle$ & $::=$ 'initial' 'algorithm' $\langle$ statement_list $\rangle$ \\
\hline$\langle$ equation_list $\rangle$ & $:=\{\langle$ equation $\rangle$ ' ;' $\}$ \\
\hline$\langle$ statement_list $\rangle$ & $:=\{\langle$ statement $\rangle$ ';' $\}$ \\
\hline$\langle$ equation $\rangle$ & $::=\langle$ equation_1 $\rangle\langle$ comment $\rangle$ \\
\hline$\langle$ equation_1 $\rangle$ & $\begin{aligned}:= & \langle\text { equality_equation }\rangle \\
\mid & \langle\text { if_equation }\rangle \\
\mid & \langle\text { for_equation }\rangle \\
\mid & \langle\text { connect_clause }\rangle \\
\mid & \langle\text { when_equation }\rangle \\
\mid & \langle\text { name }\rangle \text { function_call_args }\rangle\end{aligned}$ \\
\hline$\langle$ statement $\rangle$ & $::=\langle$ statement_1 $\rangle\langle$ comment $\rangle$ \\
\hline$\langle$ statement_1 $\rangle$ & $\begin{array}{l}::=\langle\text { assignment_statement }\rangle \\
\mid \quad\langle\text { component_reference }\rangle\langle\text { function_call_args }\rangle\end{array}$ \\
\hline
\end{tabular}




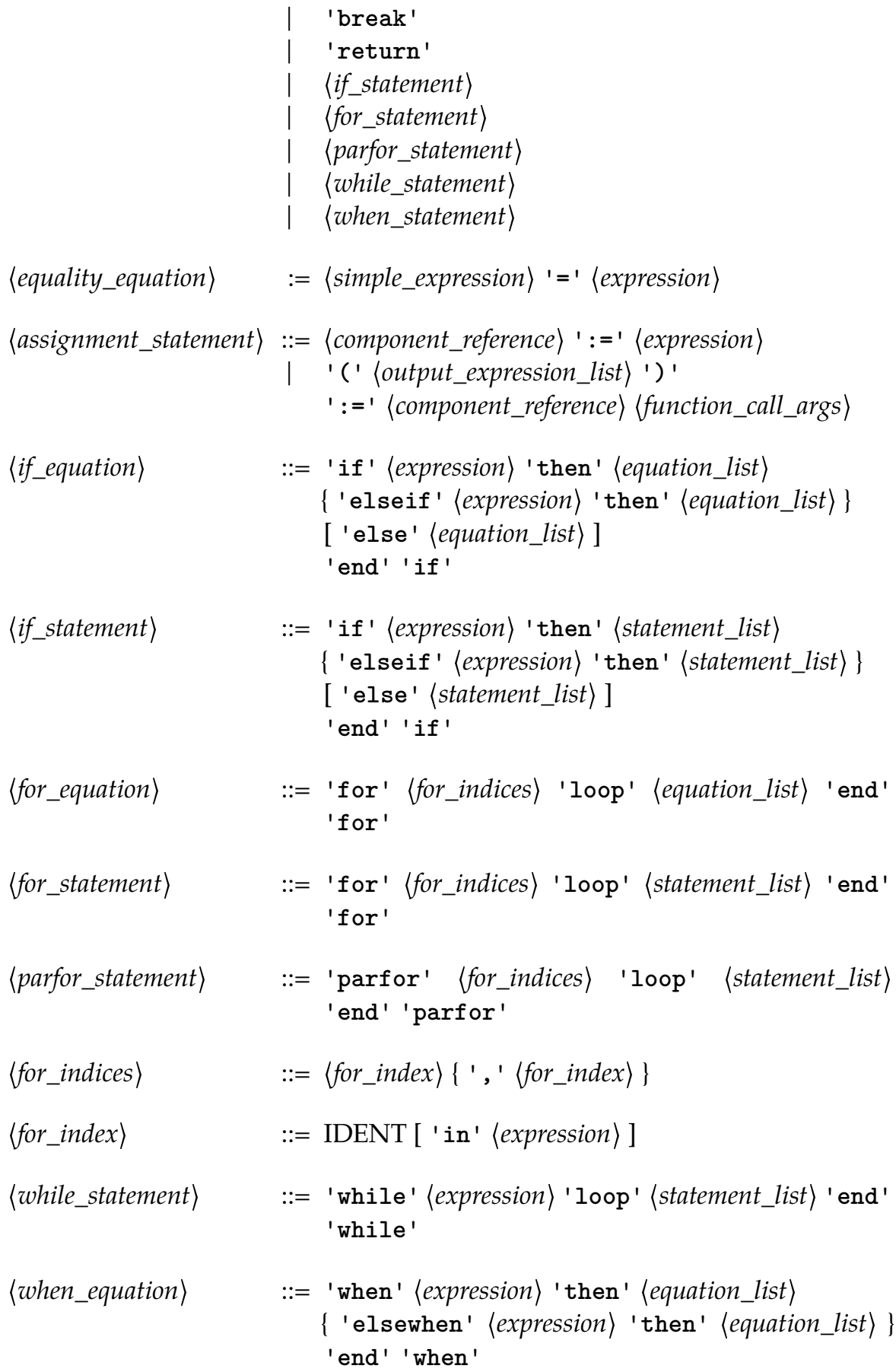




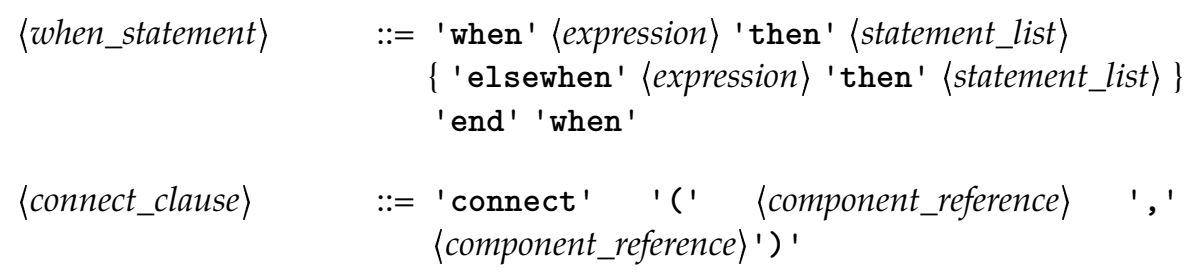

\subsubsection{Expressions}

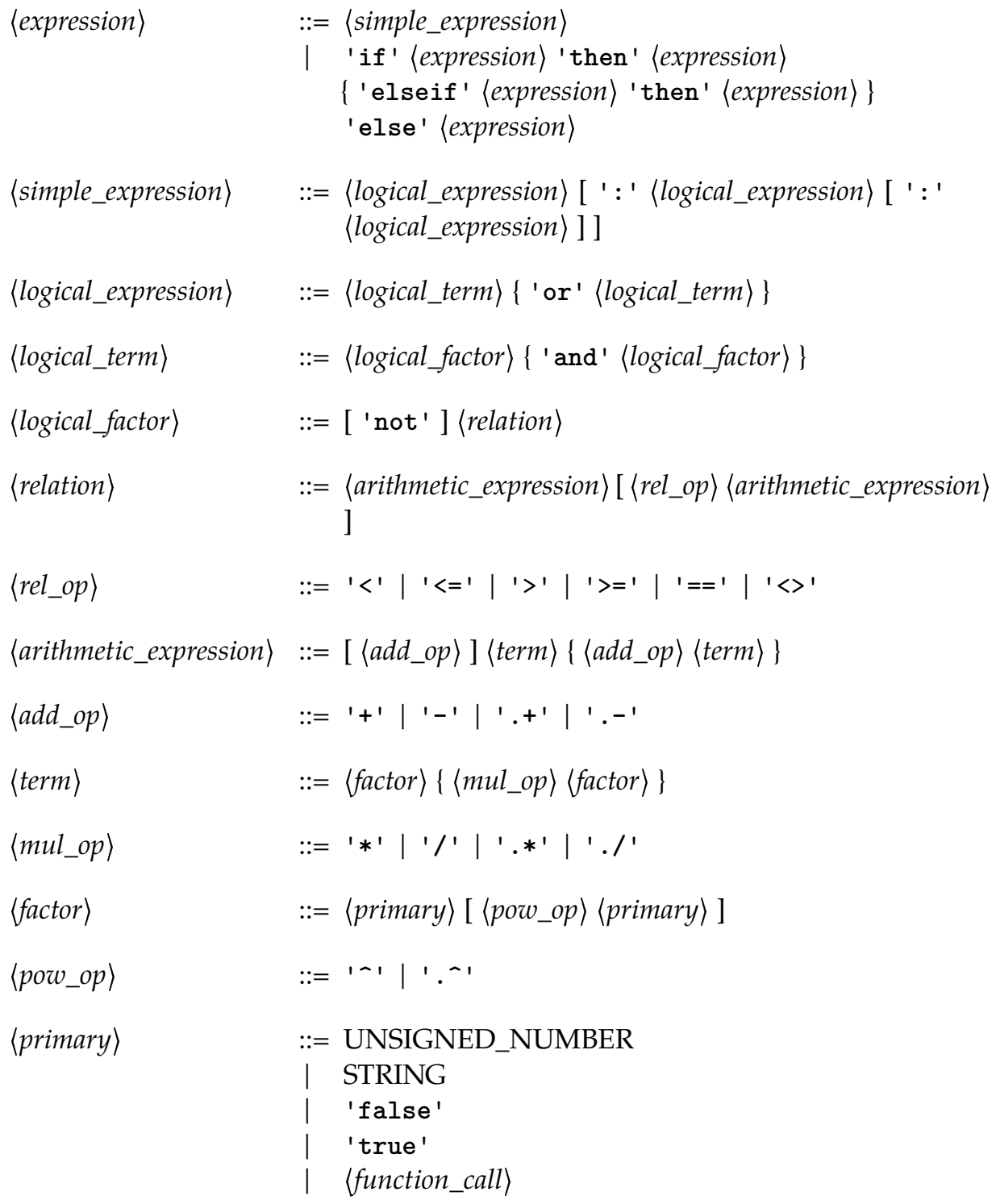




$$
\begin{aligned}
& \text { | 〈component_reference } \\
& \text { | '('〈output_expression_list ' ')' } \\
& \text { | ' ['〈expression_list }\rangle\{\text { ';'〈expression_list }\rangle\} \text { ']' } \\
& \text { | '\{' (function_arguments }\rangle \text { ' }\} \text { ' } \\
& \text { 'end' } \\
& \langle\text { function_call }\rangle \\
& ::=\langle\text { name }\rangle\langle\text { function_call_args }\rangle \\
& \text { | 'der' (function_call_args) } \\
& \text { 'initial' 〈function_call_args) } \\
& \langle\text { name }\rangle \\
& ::=[\text { ' . ' }] \text { IDENT }\{\text { ' . IDENT }\} \\
& \langle\text { component_reference }\rangle \quad:=[\text { [ .' ] IDENT [ } \\
& \{\text { '. ' IDENT [ }\langle\text { array_subscripts }\rangle]\} \\
& \left\langle\text { function_call_args } \quad::={ }^{\prime}(\text { [ }[\text { function_arguments }\rangle]\right. \text { ' )' } \\
& \langle\text { function_arguments }\rangle \quad:=\langle\text { function_argument }\rangle[\text { ', ' 〈function_arguments }\rangle \text { | } \\
& \langle\text { for for_indices }\rangle] \\
& \text { | 〈named_arguments } \\
& \langle\text { named_arguments }\rangle \quad::=\langle\text { named_argument }\rangle[\text { ', '〈named_arguments }\rangle] \\
& \langle\text { named_argument }\rangle \quad::=\text { IDENT ' }=\text { ' function_argument }\rangle \\
& \langle\text { function_argument } \quad::=\text { expression } \\
& \text { | 'function' }\langle\text { name }\rangle \text { ' (' [ }\langle\text { named_arguments }\rangle \text { ] ')' } \\
& \langle\text { output_expression_list }\rangle::=[\langle\text { expression }\rangle]\left\{\mathrm{I}^{\prime},[\langle\text { expression }\rangle]\right\} \\
& \langle\text { expression_list }\rangle \quad::=\langle\text { expression }\rangle\left\{{ }^{\prime},{ }^{\prime}\langle\text { expression }\rangle\right\} \\
& \left.\langle\text { array_subscripts }\rangle \quad::={ }^{\prime}\left[\text { ' }\langle\text { subscript }\rangle\left\{{ }^{\prime},{ }^{\prime}\langle\text { subscript }\rangle\right\}\right]^{\prime}\right]^{\prime} \\
& \langle\text { subscript } \quad::=\text { : } \\
& \text { | }\langle\text { expression }\rangle \\
& \langle\text { comment }\rangle \quad::=\langle\text { string_comment }\rangle[\langle\text { annotation }\rangle] \\
& \langle\text { string_comment }\rangle \quad::=[\text { STRING }\{+ \text { STRING }\}] \\
& \langle\text { annotation }\rangle \quad::=\text { 'annotation' 〈class_modification }
\end{aligned}
$$





\section{Selected ParModExp OpenCL Library API definitions}

\begin{tabular}{|c|c|}
\hline char* load_source_file(const char* fileName) & Reads kernels from a file. \\
\hline void ocl_initialize() & Initializes OCL environment on first call. \\
\hline void ocl_get_device() & Gets available OCL-enabled devices. \\
\hline void ocl_create_context_and_comm_queue() & $\begin{array}{l}\text { Creates context and command queues necessary for } \\
\text { launching kernel. }\end{array}$ \\
\hline $\begin{array}{l}\text { cl_kernel ocl_create_kernel(cl_program program, const char* ker- } \\
\text { nel_name) }\end{array}$ & Extracts and creates a kernel from a given program. \\
\hline void ocl_set_kernel_args(cl_kernel kernel, int count, ...) & $\begin{array}{l}\text { Sets Kernel arguments. Count is the number of argu- } \\
\text { ments being passed. }\end{array}$ \\
\hline void ocl_execute_kernel(cl_kernel kernel) & Executes a kernel. \\
\hline cl_program ocl_build_p_from_src(const char* source, int isfile) & $\begin{array}{l}\text { Builds a program from a source file containing Kernels } \\
\text { or from a text buffer if isfile }=0 .\end{array}$ \\
\hline cl_mem ocl_alloc_init(void* src_data, size_t size) & $\begin{array}{l}\text { Allocates memory space on device and returns the } \\
\text { handle to the buffer object also initializes if from host } \\
\text { memory IF src_data is not NULL. Size is the actual size } \\
\text { in bytes. }\end{array}$ \\
\hline $\begin{array}{l}\text { void ocl_create_execution_memory_buffer(device_buffer* } \\
\text { d_buff) }\end{array}$ & $\begin{array}{l}\text { This function allocates a large amount of memory } \\
\text { to be used for creating arrays inside parallel func- } \\
\text { tions. If this fails try reducing the amount by in- } \\
\text { creasing the \#define OCL_BUFFER_SIZE_FRACTION } \\
\text { which defines the fraction of memory from the avail- } \\
\text { able GLOBAL_MEM_SIZE to be used as buffer. }\end{array}$ \\
\hline $\begin{array}{l}\text { cl_mem ocl_alloc_init_real_arr(modelica_real* host_array, int } \\
\text { a_size) }\end{array}$ & $\begin{array}{l}\text { Allocates a double array on device and returns the han- } \\
\text { dle to the buffer object also initializes it from host ar- } \\
\text { ray IF host array is not NULL. Use size } 1 \text { to allocate a } \\
\text { Scalar. }\end{array}$ \\
\hline
\end{tabular}


cl_mem ocl_alloc_init_integer_arr(modelica_integer* host_array, int a_size)
Allocates an integer array on device and returns the handle to the buffer object also initializes if from host array IF host array is not NULL. Use size 1 to allocate a Scalar.

\begin{tabular}{|c|c|}
\hline $\begin{array}{l}\text { void ocl_copy_device_to_device_real(cl_mem dev_src_array, } \\
\text { cl_mem device_dest_array, int a_size) }\end{array}$ & Copies one real buffer to another on the device. \\
\hline $\begin{array}{l}\text { void ocl_copy_device_to_device_integer(cl_mem dev_src_array, } \\
\text { cl_mem device_dest_array, int a_size) }\end{array}$ & Copies one integer buffer to another on the device. \\
\hline $\begin{array}{l}\text { void ocl_copy_back_to_host_real(cl_mem dev_output_array, } \\
\text { modelica_real* }{ }^{*} \text { dest_host_array, int a_size) }\end{array}$ & Copies a double array back to host. \\
\hline $\begin{array}{l}\text { void ocl_copy_back_to_host_integer(cl_mem dev_output_array, } \\
\text { modelica_integer* dest_host_array, int a_size) }\end{array}$ & Copies an integer array back to host. \\
\hline $\begin{array}{l}\text { void ocl_copy_to_device_real(cl_mem dev_dest_array, model- } \\
\text { ica_real* src_host_array, int a_size) }\end{array}$ & $\begin{array}{l}\text { Copies a double array to already allocated device } \\
\text { buffer. }\end{array}$ \\
\hline $\begin{array}{l}\text { void ocl_copy_to_device_real(cl_mem dev_dest_array, model- } \\
\text { ica_real* src_host_array, int a_size) }\end{array}$ & $\begin{array}{l}\text { Copies an integer array to ALREADY allocated device } \\
\text { buffer. }\end{array}$ \\
\hline void ocl_error_check(int operation, cl_int error_code) & Checks error codes. \\
\hline $\begin{array}{l}\text { void ocl_real_matrix_matrix_matrix(const } \\
\text { op,modelica_real }{ }^{*} \text { src_1, int } \mathrm{M} \text {, modelica_real }{ }^{*} \text { src_2, int } \mathrm{N} \text {, } \\
\left.\text { modelica_real }{ }^{*} \text { dest, int } \mathrm{K}\right)\end{array}$ & $\begin{array}{l}\text { Matrix-matrix operations returning a matrix. Used for } \\
\text { easy arrangement of built-in functions. }\end{array}$ \\
\hline $\begin{array}{l}\text { void ocl_real_matrix_matrix(const char* op, modelica_real* } \\
\text { src_1,int } \mathrm{M} \text {,int } \mathrm{N} \text {, modelica_real }{ }^{*} \text { dest) }\end{array}$ & $\begin{array}{l}\text { Matrix operations returning a matrix. Used for easy } \\
\text { arrangement of built-in functions. }\end{array}$ \\
\hline void ocl_clean_up () & Cleaning up operations \\
\hline size_t modelica_array_nr_of_elements(base_array_t *a) & $\begin{array}{l}\text { Returns the number of elements in a given modelica } \\
\text { base host array. }\end{array}$ \\
\hline size_t device_array_nr_of_elements(device_array *a) & $\begin{array}{l}\text { Returns the number of elements in a given modelica } \\
\text { base device array. }\end{array}$ \\
\hline $\begin{array}{l}\text { cl_mem ocl_device_alloc_init(modelica_integer* host_array, } \\
\text { size_t size) }\end{array}$ & $\begin{array}{l}\text { Allocates memory on parallel device and initializes the } \\
\text { memory from the given host Integer array. }\end{array}$ \\
\hline $\begin{array}{l}\text { cl_mem ocl_device_alloc_init(modelica_real }{ }^{*} \text { host_array, size_t } \\
\text { size) }\end{array}$ & $\begin{array}{l}\text { Allocates memory on parallel device and initializes the } \\
\text { memory from the given host Real array. }\end{array}$ \\
\hline cl_mem ocl_device_alloc(size_t size) & Allocates memory buffer on parallel device. \\
\hline
\end{tabular}




\section{Bibliography}

[1] Equa AB. IDA Simulation Environment. 2018. uRL: http : / /www . equa . se/eng. se.html (visited on Nov. 29, 2018).

[2] Advanced Micro Devices. OpenCL Static C++ Kernel Language Extension. 2013. URL: http : / / amd-dev . wpengine . netdna - cdn . com / wordpress/media/2012/10/CPP_kernel_language.pdf (visited on Nov. 29, 2018).

[3] Advanced Micro Devices, Inc. The AMD OpenCL Zone. 2013. urL: http: //developer.amd.com/tools-and-sdks/opencl-zone/ (visited on Nov. 29, 2018).

[4] Johan Åkesson. "Optimica an extension of Modelica Supporting Dynamic Optimization". In: Proceedings of the 6th International Modelica Conference. Ed. by Bernhard Bachmann. Bielefeld, Germany: Modelica Association, Mar. 2008.

[5] Johan Åkesson, K-E Årzén, Magnus Gäfvert, Tove Bergdahl, and Hubertus Tummescheit. "Modeling and optimization with Optimica and JModelica. org-Languages and tools for solving large-scale dynamic optimization problems". In: Computers $\mathcal{E}$ Chemical Engineering 34.11 (2010), pp. 1737-1749.

[6] Helmut Alt, Norbert Blum, Kurt Mehlhorn, and Markus Paul. “Computing a maximum cardinality matching in a bipartite graph in time o (n1. 5mlog n)". In: Information Processing Letters 37.4 (1991), pp. 237240. 
[7] Peter Aronsson. "Automatic Parallelization of Equation-Based Simulation Programs". Doctoral thesis No 1022. Department of Computer and Information Science: Linköping University, June 2006. IsBN: 9185523-68-2. uRL: http : / / urn . kb . se / resolve ? urn=urn : nbn : se : liu:diva-7446 (visited on Oct. 7, 2011).

[8] Modelica Association et al. "Modelica Standard library". In: Online, URL: http://www. modelica. org/libraries/Modelica (2008).

[9] Modelica Association et al. "Modelica-A Unified Object-Oriented Language for Physical Systems Modeling-Language Specification Version 3.3 Revision 1, 2014". In: URL http://www. modelica. org ().

[10] Sochacki Bartosz. "The OpenCL C++ specification, version: 1.0, document revision". In: Khronos OpenCL Working Group (2016).

[11] Federico Bergero, Xenofon Floros, Joaquín Fernández, Ernesto Kofman, and François E Cellier. "Simulating Modelica models with a stand-alone quantized state systems solver". In: Proceedings of the 9th International Modelica Conference. Ed. by Martin Otter and Dirk Zimmer. Munich, Germany: Linköping University Electronic Press, Sept. 2012. DOI: $10.3384 /$ ecp 12076 .

[12] Blaise Barney, Lawrence Livermore National Laboratory. OpenMP). 2018. URL: https : / computing . llnl . gov/tutorials / openMP/ (visited on Nov. 29, 2018).

[13] Blaise Barney, Lawrence Livermore National Laboratory. POSIX Threads Programming). 2018. uRL: https : / / computing . llnl . gov / tutorials/pthreads/ (visited on Nov. 29, 2018).

[14] Hans-J. Boehm, Alan J. Demers, and Scott Shenker. "Mostly parallel garbage collection". In: SIGPLAN Not. 26.6 (May 1991), pp. 157-164. ISSN: 0362-1340. DOI: 10.1145/113446.113459.

[15] Hans-Juergen Boehm and Mark Weiser. "Garbage collection in an uncooperative environment". In: Software: Practice and Experience 18.9 (1988), pp. 807-820. Issn: 1097-024X. DOI: 10.1002/spe. 4380180902.

[16] Boost.org. Boost C++ Libraries. 2015. URL: http : / / www . boost . org/ (visited on Nov. 29, 2018).

[17] Dag Brück, Hilding Elmqvist, Sven Erik Mattsson, and Hans Olsson. "Dymola for multi-engineering modeling and simulation". In: Proceedings of the 2nd International Modelica Conference. Ed. by Martin Otter. Oberpfaffenhofen, Germany: Modelica Association, Mar. 2002.

[18] J.D. Burton, K.A. Edge, and C.R. Burrows. "Partitioned Simulation of Hydraulic Systems Using Transmission-Line Modelling". In: ASME WAM. New Orleans, USA, 1993. 
[19] Francesco Casella. "A Strategy for Parallel Simulation of Declarative Object-Oriented Models of Generalized Physical Networks". In: Proceedings of the 5th International Workshop on Equation-Based Object-Oriented Modeling Languages and Tools. Ed. by Henrik Nilsson. Nottingham, UK: Linköping University Electronic Press, Apr. 2013. uRL: http: //www.ep.liu.se/ecp_home/index.en.aspx?issue=084 (visited on Mar. 31, 2013).

[20] Francesco Casella. "Simulation of Large-Scale Models in Modelica: State of the Art and Future Perspectives". In: Proceedings of the 11th International Modelica Conference, Versailles, France, September 21-23, 2015. Ed. by Peter Fritzson and Hilding Elmqvist. 118. Versailles, France: Linköping University Electronic Press, Linköpings universitet, 2015, pp. 459-468. DoI: 10.3384/ecp15118.

[21] François E Cellier, Ernesto Kofman, Gustavo Migoni, and Mario Bortolotto. "Quantized State System Simulation". In: Proc. GCMS'08, Grand Challenges in Modeling and Simulation (2008), pp. 504-510.

[22] Tim Davis, WW Hager, and IS Duff. "SuiteSparse". In: URL: faculty. cse. tamu. edu/davis/suitesparse. html (2014).

[23] Efim A Dinic. "Algorithm for solution of a problem of maximum flow in networks with power estimation". In: Soviet Math. Doklady. Vol. 11. 1970, pp. 1277-1280.

[24] Jack J Dongarra, James R Bunch, Cleve B Moler, and Gilbert W Stewart. LINPACK users' guide. Vol. 8. Siam, 1979.

[25] György Dósa. “The tight bound of first fit decreasing bin-packing algorithm is FFD $(\mathrm{I}) \leq 11 / 9$ OPT $(\mathrm{I})+6 / 9^{\prime \prime}$. In: Proceedings of the First international conference on Combinatorics, Algorithms, Probabilistic and Experimental Methodologies. Springer-Verlag. 2007, pp. 1-11.

[26] Jack Edmonds and Richard M Karp. "Theoretical improvements in algorithmic efficiency for network flow problems". In: Journal of the ACM (JACM) 19.2 (1972), pp. 248-264.

[27] Hilding Elmqvist. "A structured model language for large continuous systems". PhD thesis. Lund Institute of Technology, 1978.

[28] Hilding Elmqvist, Sven Erik Matsson, and Hans Olsson. "Parallel Model Execution on Many Cores". In: Proceedings of the 10th International Modelica Conference. Ed. by Hubertus Tummescheit and KarlErik Årzén. Lund, Sweden: Modelica Association and Linköping University Electronic Press, 2014. Dor: 10.3384/ecp14096363.

[29] Hilding Elmqvist, Sven Erik Mattsson, and Martin Otter. "Modelica-a language for physical system modeling, visualization and interaction". In: Computer Aided Control System Design, 1999. Proceedings of the 1999 IEEE International Symposium on. IEEE. 1999, pp. 630-639. 
[30] Björn Eriksson, Peter Nordin, and Petter Krus. "Hopsan, A C++ Implementation Utilising TLM Simulation Technique". In: Proceedings of the 51st Conference on Simulation and Modelling (SIMS). Oulu, Finland, Oct. 2010.

[31] Joaquín Fernández and Ernesto Kofman. "A stand-alone quantized state system solver for continuous system simulation". In: Simulation 90.7 (2014), pp. 782-799.

[32] Joaquín Fernandez, Ernesto Kofman, and Federico Bergero. "A parallel Quantized State System Solver for ODEs". In: Journal of Parallel and Distributed Computing 106 (2017), pp. 14-30.

[33] Xenofon Floros. "Exploiting model structure for efficient hybrid dynamical systems simulation". PhD thesis. ETH Zurich, 2014.

[34] Xenofon Floros, Federico Bergero, François E Cellier, and Ernesto Kofman. "Automated Simulation of Modelica Models with QSS Methods: The Discontinuous Case". In: Proceedings of the 8th International Modelica Conference. Ed. by Christoph Clauß. Dresden, Germany: Linköping University Electronic Press, Mar. 2011. Dor: 10.3384/ecp11063.

[35] Peter Fritzson. Introduction to Modeling and Simulation of Technical and Physical Systems with Modelica. John Wiley \& Sons, 2011.

[36] Peter Fritzson. Principles of Object-Oriented Modeling and Simulation with Modelica 3.3: A Cyber-Physical Approach. 2nd ed. Wiley-IEEE Press, Apr. 2015. 1256 pp. ISBN: 978-1-118-85912-4.

[37] Peter Fritzson, Peter Aronsson, Håkan Lundvall, Kaj Nyström, Adrian Pop, Levon Saldamli, and David Broman. "The OpenModelica Modeling, Simulation, and Software Development Environment". In: Simulation News Europe 44.45 (Dec. 2005).

[38] Peter Fritzson, Peter Aronsson, Adrian Pop, Hakan Lundvall, Kaj Nystrom, Levon Saldamli, David Broman, and Anders Sandholm. "OpenModelica-A free open-source environment for system modeling, simulation, and teaching". In: Computer Aided Control System Design, 2006 IEEE International Conference on Control Applications, 2006 IEEE International Symposium on Intelligent Control, 2006 IEEE. IEEE. 2006, pp. 1588-1595.

[39] Peter Fritzson and Peter Bunus. "Modelica-a general object-oriented language for continuous and discrete-event system modeling and simulation". In: Simulation Symposium, 2002. Proceedings. 35th Annual. IEEE. 2002, pp. 365-380. 
[40] Peter Fritzson, Johan Gunnarsson, and Mats Jirstrand. "MathModelica-an extensible modeling and simulation environment with integrated graphics and literate programming". In: Proceedings of the 2 nd International Modelica Conference. Ed. by Martin Otter. Oberpfaffenhofen, Germany: Modelica Association, Mar. 2002.

[41] Peter Fritzson, Adrian Pop, Adeel Asghar, Bernhard Bachmann, Willi Braun, Robert Braun, Lena Buffoni, Francesco Casella, Rodrigo Castro, Alejandro Danós, Rüdiger Franke, Mahder Gebremedhin, Bernt Lie, Alachew Mengist, Kannan Moudgalya, Lennart Ochel, Arunkumar Palanisamy, Wladimir Schamai, Martin Sjölund, Bernhard Thiele, Volker Waurich, and Per Östlund. "The OpenModelica Integrated Modeling, Simulation and Optimization Environment". In: Proceedings of the 1st American Modelica Conference. Cambridge, MA, USA: Linköping University Electronic Press, 2018.

[42] Peter Fritzson, Adrian Pop, and Martin Sjölund. Towards Modelica 4 Meta-Programming and Language Modeling with MetaModelica 2.0. Tech. rep. 2011:10. Linköping University, The Institute of Technology, 2011, p. 297.

[43] Peter Fritzson, Adrian Pop, Martin Sjölund, Per Östlund, Adeel Asghar, Willi Braun, Jens Frenkel, Lennart Ochel, Mahder Gebremedhin, Lena Buffoni, Peter Aronsson, Mikael Axin, Bernhard Bachmann, Bernhard Thiele, Vasile Baluta, Robert Braun, David Broman, Stefan Brus, Francesco Casella, Filippo Donida, Anand Ganeson, Marcus Walther, Volker Waurich, Christian Schubert, Pavel Grozman, Daniel Hedberg, Michael Hanke, Alf Isaksson, Kim Jansson, Daniel Kanth, Tommi Karhela, Juha Kortelainen, Abhinn Kothari, Petter Krus, Alexey Lebedev, Oliver Lenord, Ariel Liebman, Rickard Lindberg, Håkan Lundvall, Abhi Raj Metkar, Eric Meyers, Tuomas Miettinen, Afshin Moghadam, Maroun Nemer, Hannu Niemistö, Peter Nordin, Kristoffer Norling, Arunkumar Palanisamy, Karl Pettersson, Pavol Privitzer, Jhansi Reddy, Reino Ruusu, Per Sahlin, Wladimir Schamai, Gerhard Schmitz, Alachew Shitahun, Anton Sodja, Ingo Staack, Kristian Stavåker, Sonia Tariq, Mohsen Torabzadeh Tari, Parham Vasaiely, Niklas Worschech, Robert Wotzlaw, Björn Zackrisson, Azam Zia, et al. OpenModelica Users Guide. 2018. urL: https : / / openmodelica . org/ (visited on Nov. 29, 2018).

[44] Komei Fukuda and Tomomi Matsui. "Finding all minimum-cost perfect matchings in Bipartite graphs". In: Networks 22.5 (1992), pp. 461468.

[45] Komei Fukuda and Tomomi Matsui. "Finding all the perfect matchings in bipartite graphs". In: Applied Mathematics Letters 7.1 (1994), pp. 15-18. 
[46] Edgar Gabriel, Graham E Fagg, George Bosilca, Thara Angskun, Jack J Dongarra, Jeffrey M Squyres, Vishal Sahay, Prabhanjan Kambadur, Brian Barrett, Andrew Lumsdaine, et al. "Open MPI: Goals, concept, and design of a next generation MPI implementation". In: European Parallel Virtual Machine/Message Passing Interface Users' Group Meeting. Springer. 2004, pp. 97-104.

[47] Michael R. Garey and David S. Johnson. Computers and Intractability; A Guide to the Theory of NP-Completeness. New York, NY, USA: W. H. Freeman \& Co., 1990. ISBN: 0716710455.

[48] Mahder Gebremedhin, Afshin Moghadam, Peter Fritzson, and Kristian Stavåker. "A Data-Parallel Algorithmic Modelica Extension for Efficient Execution on Multi-Core Platforms". In: Proceedings of the 9th International Modelica Conference. Ed. by Martin Otter and Dirk Zimmer. Munich, Germany: Linköping University Electronic Press, Sept. 2012. DOI: 10.3384 /ecp12076393.

[49] CD Godsil. "Inverses of trees". In: Combinatorica 5.1 (1985), pp. 33-39.

[50] Ronald L. Graham. "Bounds on multiprocessing timing anomalies". In: SIAM journal on Applied Mathematics 17.2 (1969), pp. 416-429.

[51] Afshin Hemmati Moghadam. "Modelica PARallel benchmark suite (MPAR) - a test suite for evaluating the performance of parallel simulations of Modelica models". MA thesis. Linköping University, PELAB - Programming Environment Laboratory, 2011, p. 96.

[52] Alan C Hindmarsh, Radu Serban, and Aaron Collier. "User Documentation for IDA v3. 1.1 (sundials v3. 1.1)”. In: (2018).

[53] Alan C Hindmarsh and Allan G Taylor. PVODE and KINSOL: Parallel software for differential and nonlinear systems. Tech. rep. Lawrence Livermore National Lab., CA (United States), 1998.

[54] John E Hopcroft and Richard M Karp. "An $n^{5 / 2}$ algorithm for maximum matchings in bipartite graphs". In: SIAM Journal on computing 2.4 (1973), pp. 225-231.

[55] Intel Corporation. OpenCL Technology - Intel Developer Zone. 2013. URL: https : / / software. intel . com/en-us/intel-opencl (visited on Nov. 29, 2018).

[56] Intel Corporation. Threading Building Blocks (IntelßTBB). 2015. URL: https : / / www . threadingbuildingblocks . org/ (visited on Nov. 29, 2018).

[57] Iso.org. ISO/IEC 9899:1999-Programming languages - C. 2011. uRL: http: //www . iso.org/iso/iso_catalogue/catalogue_ics/catalogue_ detail_ics.htm?csnumber=29237 (visited on Nov. 29, 2018).

[58] David S Johnson. "Near-optimal bin packing algorithms". PhD thesis. Massachusetts Institute of Technology, 1973. 
[59] David S. Johnson, Alan Demers, Jeffrey D. Ullman, Michael R Garey, and Ronald L. Graham. "Worst-case performance bounds for simple one-dimensional packing algorithms". In: SIAM Journal on Computing 3.4 (1974), pp. 299-325.

[60] Christoph W Keßler. "NestStep: nested parallelism and virtual shared memory for the BSP model". In: The Journal of Supercomputing 17.3 (2000), pp. 245-262.

[61] Christoph Kessler, Peter Fritzson, and Mattias Eriksson. "NestStepModelica-Mathematical Modeling and Bulk-Synchronous Parallel Simulation". In: International Workshop on Applied Parallel Computing. Springer. 2006, pp. 1006-1015.

[62] Khronos Group. Conformant Products. 2015. uRL: https : / / www . khronos . org / conformance / adopters / conformant - products / opencl (visited on Nov. 29, 2018).

[63] Khronos Group. OpenGL - The Industry Standard for High Performance Graphics. 2015. URL: https : / / www . opengl . org/ (visited on Nov. 29, 2018).

[64] Khronos OpenCL Working Group. The OpenCL Specification. Version: 1.0 Document Revision: 29. 2012. URL: https : / / www . khronos . org / registry/cl/specs/opencl-1.0.29.pdf (visited on Nov. 29, 2018).

[65] Ernesto Kofman and Sergio Junco. "Quantized-state systems: a DEVS Approach for continuous system simulation". In: Transactions of The Society for Modeling and Simulation International 18.3 (2001), pp. 123132.

[66] Richard E Korf. "Multi-Way Number Partitioning." In: IJCAI. 2009, pp. 538-543.

[67] Arnold R Krommer. Numerical integration: on advanced computer systems. Vol. 848. Springer Science \& Business Media, 1994.

[68] Petter Krus. "Robust System Modelling Using Bi-lateral Delay Lines". In: Proceedings of the 2nd Conference on Modeling and Simulation for Safety and Security (SimSafe). Linköping, Sweden, 2005.

[69] Petter Krus, Robert Braun, Peter Nordin, Björn Eriksson, et al. HopSAN project. 2015. URL: http : / / www . iei . liu . se / flumes / systemsimulation/hopsan (visited on Apr. 11, 2015).

[70] Randall J LeVeque. Finite difference methods for ordinary and partial differential equations: steady-state and time-dependent problems. Vol. 98. Siam, 2007.

[71] John H Lienhard. A heat transfer textbook. Courier Corporation, 2013. 
[72] Håkan Lundvall. "Automatic Parallelization using Pipelining for Equation-Based Simulation Languages". Licentiate thesis No 1381. Department of Computer and Information Science: Linköping University, 2008. ISBN: 978-91-7393-799-3. uRL: http: //urn . kb. se/resolve? urn=urn:nbn:se: liu:diva-12504 (visited on Oct. 7, 2011).

[73] Håkan Lundvall, Kristian Stavåker, Peter Fritzson, and Christoph Kessler. "Automatic Parallelization of Simulation Code for Equationbased Models with Software Pipelining and Measurements on Three Platforms". In: Computer Architecture News 36.5 (2008).

[74] MapleSoft. MapleSim. 2018. urL: http: //www.maplesoft.com (visited on Nov. 29, 2018).

[75] Wolfram Mathcore. Wolfram System Modeler. 2018. uRL: http : / / www . wolfram.com/system-modeler (visited on Nov. 29, 2018).

[76] MathWorks. Parallel Computing Toolbox. 2018. urL: https : / / se . mathworks . com/products / parallel-computing . html (visited on Nov. 29, 2018).

[77] Microsoft. Developing games - Windows app development. 2015. URL: https://msdn.microsoft.com/en-us/library/windows/desktop/ $\mathrm{ff} 476331 \% 28 \mathrm{v}=\mathrm{vs} .85 \% 29$. aspx (visited on Nov. 29, 2018).

[78] Microsoft. DirectX. 2015. urL: https : / / msdn . microsoft . com / library/windows/apps/hh452744 (visited on Nov. 29, 2018).

[79] Martin Otter, ed. Proceedings of the 2nd International Modelica Conference. Oberpfaffenhofen, Germany: Modelica Association, Mar. 2002.

[80] Martin Otter and Dirk Zimmer, eds. Proceedings of the 9th International Modelica Conference. Munich, Germany: Linköping University Electronic Press, Sept. 2012. Dor: 10.3384/ecp12076.

[81] Hubertus Tummescheit and Karl-Erik Årzén, eds. Proceedings of the 10th International Modelica Conference. Lund, Sweden: Modelica Association and Linköping University Electronic Press, Mar. 2014. DOI: 10 . 3384/ecp14096.

[82] Nvidia. Nvidia CUDA Compute Unified Device Architecture programming guide. Version: 2.0. 2008.

[83] NVIDIA Corporation. NVIDIA OpenCL. 2015. urL: https : / / developer. nvidia. com/opencl (visited on Nov. 29, 2018).

[84] Lennart Ochel, Robert Braun, Bernhard Thiele, Adeel Asghar, Lena Buffoni, Magnus Eek, Peter Fritzson, Dag Fritzson, Sune Horkeby, Robert Hällquist, Åke Kinnander, Arunkumar Palanisamy, Adrian Pop, and Martin Sjölund. "OMSimulator - Integrated FMI and TLMbased Co-simulation with Composite Model Editing and SSP". In: Proceedings of the 13th International Modelica Conference. OTH Regensburg, 
Germany: Modelica Association and Linköping University Electronic Press, 2019.

[85] GNU Octave. Parallel package. 2018. uRL: https://wiki. octave .org/ Parallel_package (visited on Nov. 29, 2018).

[86] Per Östlund. "Simulation of Modelica Models on the CUDA Architecture". MA thesis. Department of Computer and Information Science: Linköping University, Nov. 2009. uRL: http : / /urn . kb . se/resolve? urn=urn:nbn:se:liu:diva-52060.

[87] Per Östlund, Kristian Stavåker, and Peter Fritzson. "Parallel Simulation of Equation-Based Models on CUDA-Enabled GPUs". In: Proceedings of the 9th Workshop on Parallel/High-Performance Object-Oriented Scientific Computing. Reno, Nevada: ACM, 2010. Dor: 10 .1145/2039312. 2039317.

[88] Linda R Petzold. Description of DASSL: a Differential/Algebraic System Ssolver. Tech. rep. Sandia National Labs., Livermore, CA (USA), 1982.

[89] Adrian Pop and Peter Fritzson. "MetaModelica: A unified equationbased semantical and mathematical modeling language". In: Modular Programming Languages. Springer, 2006, pp. 211-229.

[90] WH Press, SA Teukolsky, WT Vetterling, and BP Flannery. “Numerical Recipes in Fortran 77: The Art of Scientific Computing. Cambridge Univ". In: Press, Cambridge (1992).

[91] Thomas Rauber and Gudula Rünger. "Parallel execution of embedded and iterated Runge-Kutta methods". In: Concurrency: Practice and Experience 11.7 (1999), pp. 367-385.

[92] Wolfram Research. Parallel Computing. 2018. uRL: https://reference. wolf ram . com/language/guide / ParallelComputing . html (visited on Nov. 29, 2018).

[93] Wolfram Research. Parallel Computing Tools User Guide. 2018. URL: https : / / reference . wolfram . com / language / ParallelTools / tutorial/Overview .html (visited on Nov. 29, 2018).

[94] Conrad Sanderson. "Armadillo: An open source C++ linear algebra library for fast prototyping and computationally intensive experiments". In: (2010).

[95] E Schikuta. "Message-passing-interface-forum: MPI: A MessagePassing Interface Standard". In: Techn. Ber., University of Tennessee, Knoxville, Tennesee (1994).

[96] Alexander Siemers, Dag Fritzson, and Peter Fritzson. "Meta-Modeling for Multi-physics Co-simulations applied for OpenModelica". In: Proceedings of International Congress on Methodologies for Emerging Technologies in Automation (ANIPLA). 2006. 
[97] ITI GmbH. SimulationX. Simulation X. 2018. uRL: http://www . itisim. com/simulationx.html (visited on Nov. 29, 2018).

[98] Martin Sjölund, Robert Braun, Peter Fritzson, and Petter Krus. "Towards Efficient Distributed Simulation in Modelica using Transmission Line Modeling". In: Proceedings of the 3rd International Workshop on Equation-Based Object-Oriented Modeling Languages and Tools. Ed. by Peter Fritzson, Edward Lee, François Cellier, and David Broman. Oslo, Norway: Linköping University Electronic Press, Oct. 2010, pp. 71-80. URL: http: //www.ep.liu.se/ecp/047/ (visited on Oct. 7, 2011).

[99] Alexander Souza. Combinatorial Algorithms. 2011. urL: http : / / www2 . informatik . hu - berlin . de / alcox / lehre / lvws1011 / coalg / combinatorial_algorithms.pdf (visited on Nov. 29, 2018).

[100] Michael Tiller. Modelica by Example. Xogeny, 2014.

[101] Peter J Van Der Houwen and Benjamin P Sommeijer. "Parallel iteration of high-order Runge-Kutta methods with stepsize control". In: Journal of Computational and Applied Mathematics 29.1 (1990), pp. 111-127.

[102] Marcus Walther, Volker Waurich, Christian Schubert, and Ines Gubsch. "Equation based parallelization of Modelica models". In: Proceedings of the 10th International Modelica Conference. Ed. by Hubertus Tummescheit and Karl-Erik Årzén. Lund, Sweden: Modelica Association and Linköping University Electronic Press, Mar. 2014. DoI: 10 . 3384/ecp140961213.

[103] Volker Waurich, Ines Gubsch, Christian Schubert, and Marcus Walther. "Reshuffling: A symbolic pre-processing algorithm for improved robustness, performance and parallelization for the simulation of differential algebraic equations". In: Proceedings of the 6th International Workshop on Equation-Based Object-Oriented Modeling Languages and Tools. ACM. 2014, pp. 3-10.

[104] Binzhou Xia and Zhiyi Tan. "Tighter bounds of the First Fit algorithm for the bin-packing problem". In: Discrete Applied Mathematics 158.15 (2010), pp. 1668-1675. IssN: 0166-218X. Dor: https : // doi . org/ 10 . 1016/j.dam . 2010 . 05 . 026. URL: http : / /www . sciencedirect . com/ science/article/pii/S0166218X10002088.

[105] Bernard P Zeigler and Jong Sik Lee. "Theory of quantized systems: formal basis for DEVS/HLA distributed simulation environment". In: Enabling Technology for Simulation Science II. Vol. 3369. International Society for Optics and Photonics. 1998, pp. 49-59. 
Department of Computer and Information Science

Linköpings universitet

\section{Dissertations}

\section{Linköping Studies in Science and Technology Linköping Studies in Arts and Science \\ Linköping Studies in Statistics \\ Linköping Studies in Information Science}

\section{Linköping Studies in Science and Technology}

No 14 Anders Haraldsson: A Program Manipulation System Based on Partial Evaluation, 1977, ISBN 917372-144-1.

No 17 Bengt Magnhagen: Probability Based Verification of Time Margins in Digital Designs, 1977, ISBN 91-7372157-3.

No 18 Mats Cedwall: Semantisk analys av processbeskrivningar i naturligt språk, 1977, ISBN 91- 7372168-9.

No 22 Jaak Urmi: A Machine Independent LISP Compiler and its Implications for Ideal Hardware, 1978, ISBN 91-7372-188-3.

No 33 Tore Risch: Compilation of Multiple File Queries in a Meta-Database System, 1978, ISBN 91- 7372-232-4.

No 51 Erland Jungert: Synthesizing Database Structures from a User Oriented Data Model, 1980, ISBN 917372-387-8.

No 54 Sture Hägglund: Contributions to the Development of Methods and Tools for Interactive Design of Applications Software, 1980, ISBN 91-7372-404-1.

No 55 Pär Emanuelson: Performance Enhancement in a Well-Structured Pattern Matcher through Partial Evaluation, 1980, ISBN 91-7372-403-3.

No 58 Bengt Johnsson, Bertil Andersson: The HumanComputer Interface in Commercial Systems, 1981, ISBN 91-7372-414-9.

No 69 H. Jan Komorowski: A Specification of an Abstract Prolog Machine and its Application to Partial Evaluation, 1981, ISBN 91-7372-479-3.

No 71 René Reboh: Knowledge Engineering Techniques and Tools for Expert Systems, 1981, ISBN 91-7372489-0.

No 77 Östen Oskarsson: Mechanisms of Modifiability in large Software Systems, 1982, ISBN 91- 7372-527-7.

No 94 Hans Lunell: Code Generator Writing Systems, 1983, ISBN 91-7372-652-4.

No 97 Andrzej Lingas: Advances in Minimum Weight Triangulation, 1983, ISBN 91-7372-660-5.

No 109 Peter Fritzson: Towards a Distributed Programming Environment based on Incremental Compilation, 1984, ISBN 91-7372-801-2.

No 111 Erik Tengvald: The Design of Expert Planning Systems. An Experimental Operations Planning System for Turning, 1984, ISBN 91-7372- 805-5.

No 155 Christos Levcopoulos: Heuristics for Minimum Decompositions of Polygons, 1987, ISBN 91-7870133-3.

No 165 James W. Goodwin: A Theory and System for NonMonotonic Reasoning, 1987, ISBN 91-7870-183-X.

No 170 Zebo Peng: A Formal Methodology for Automated Synthesis of VLSI Systems, 1987, ISBN 91-7870-225-9.

No 174 Johan Fagerström: A Paradigm and System for Design of Distributed Systems, 1988, ISBN 91-7870301-8.
No 192 Dimiter Driankov: Towards a Many Valued Logic of Quantified Belief, 1988, ISBN 91-7870-374-3.

No 213 Lin Padgham: Non-Monotonic Inheritance for an Object Oriented Knowledge Base, 1989, ISBN 917870-485-5.

No 214 Tony Larsson: A Formal Hardware Description and Verification Method, 1989, ISBN 91-7870-517-7.

No 221 Michael Reinfrank: Fundamentals and Logical Foundations of Truth Maintenance, 1989, ISBN 917870-546-0.

No 239 Jonas Löwgren: Knowledge-Based Design Support and Discourse Management in User Interface Management Systems, 1991, ISBN 91-7870-720-X.

No 244 Henrik Eriksson: Meta-Tool Support for Knowledge Acquisition, 1991, ISBN 91-7870-746-3.

No 252 Peter Eklund: An Epistemic Approach to Interactive Design in Multiple Inheritance Hierarchies, 1991, ISBN 91-7870-784-6.

No 258 Patrick Doherty: NML3 - A Non-Monotonic Formalism with Explicit Defaults, 1991, ISBN 917870-816-8.

No 260 Nahid Shahmehri: Generalized Algorithmic Debugging, 1991, ISBN 91-7870-828-1.

No 264 Nils Dahlbäck: Representation of DiscourseCognitive and Computational Aspects, 1992, ISBN 91-7870-850-8.

No 265 Ulf Nilsson: Abstract Interpretations and Abstract Machines: Contributions to a Methodology for the Implementation of Logic Programs, 1992, ISBN 917870-858-3.

No 270 Ralph Rönnquist: Theory and Practice of Tensebound Object References, 1992, ISBN 91-7870-873-7.

No 273 Björn Fjellborg: Pipeline Extraction for VLSI Data Path Synthesis, 1992, ISBN 91-7870-880-X.

No 276 Staffan Bonnier: A Formal Basis for Horn Clause Logic with External Polymorphic Functions, 1992, ISBN 91-7870-896-6.

No 277 Kristian Sandahl: Developing Knowledge Management Systems with an Active Expert Methodology, 1992, ISBN 91-7870-897-4.

No 281 Christer Bäckström: Computational Complexity of Reasoning about Plans, 1992, ISBN 91-7870-979-2.

No 292 Mats Wirén: Studies in Incremental Natural Language Analysis, 1992, ISBN 91-7871-027-8.

No 297 Mariam Kamkar: Interprocedural Dynamic Slicing with Applications to Debugging and Testing, 1993, ISBN 91-7871-065-0.

No302 Tingting Zhang: A Study in Diagnosis Using Classification and Defaults, 1993, ISBN 91-7871-0782.

No 312 Arne Jönsson: Dialogue Management for Natural Language Interfaces - An Empirical Approach, 1993, ISBN 91-7871-110-X.

No 338 Simin Nadjm-Tehrani: Reactive Systems in Physical Environments: Compositional Modelling and Framework for Verification, 1994, ISBN 91-7871-237-8. 
No 371 Bengt Savén: Business Models for Decision Support and Learning. A Study of Discrete-Event Manufacturing Simulation at Asea/ABB 1968-1993, 1995, ISBN 91-7871-494-X.

No 375 Ulf Söderman: Conceptual Modelling of Mode Switching Physical Systems, 1995, ISBN 91-7871-5164.

No 383 Andreas Kågedal: Exploiting Groundness in Logic Programs, 1995, ISBN 91-7871-538-5.

No 396 George Fodor: Ontological Control, Description, Identification and Recovery from Problematic Control Situations, 1995, ISBN 91-7871-603-9.

No 413 Mikael Pettersson: Compiling Natural Semantics, 1995, ISBN 91-7871-641-1.

No 414 Xinli Gu: RT Level Testability Improvement by Testability Analysis and Transformations, 1996, ISBN 91-7871-654-3.

No 416 Hua Shu: Distributed Default Reasoning, 1996, ISBN 91-7871-665-9.

No 429 Jaime Villegas: Simulation Supported Industrial Training from an Organisational Learning Perspective - Development and Evaluation of the SSIT Method, 1996, ISBN 91-7871-700-0.

No 431 Peter Jonsson: Studies in Action Planning: Algorithms and Complexity, 1996, ISBN 91-7871-7043.

No 437 Johan Boye: Directional Types in Logic Programming, 1996, ISBN 91-7871-725-6.

No 439 Cecilia Sjöberg: Activities, Voices and Arenas: Participatory Design in Practice, 1996, ISBN 91-7871728-0.

No 448 Patrick Lambrix: Part-Whole Reasoning in Description Logics, 1996, ISBN 91-7871-820-1.

No 452 Kjell Orsborn: On Extensible and Object-Relational Database Technology for Finite Element Analysis Applications, 1996, ISBN 91-7871-827-9.

No 459 Olof Johansson: Development Environments for Complex Product Models, 1996, ISBN 91-7871-855-4.

No 461 Lena Strömbäck: User-Defined Constructions in Unification-Based Formalisms, 1997, ISBN 91-7871857-0.

No 462 Lars Degerstedt: Tabulation-based Logic Programming: A Multi-Level View of Query Answering, 1996, ISBN 91-7871-858-9.

No 475 Fredrik Nilsson: Strategi och ekonomisk styrning En studie av hur ekonomiska styrsystem utformas och används efter företagsförvärv, 1997, ISBN 917871-914-3.

No 480 Mikael Lindvall: An Empirical Study of Requirements-Driven Impact Analysis in Object-Oriented Software Evolution, 1997, ISBN 91-7871-927-5.

No 485 Göran Forslund: Opinion-Based Systems: The Cooperative Perspective on Knowledge-Based Decision Support, 1997, ISBN 91-7871-938-0.

No 494 Martin Sköld: Active Database Management Systems for Monitoring and Control, 1997, ISBN 917219-002-7.

No 495 Hans Olsén: Automatic Verification of Petri Nets in a CLP framework, 1997, ISBN 91-7219-011-6.

No 498 Thomas Drakengren: Algorithms and Complexity for Temporal and Spatial Formalisms, 1997, ISBN 917219-019-1.

No 502 Jakob Axelsson: Analysis and Synthesis of Heterogeneous Real-Time Systems, 1997, ISBN 91-7219-035-3.
No 503 Johan Ringström: Compiler Generation for DataParallel Programming Languages from Two-Level Semantics Specifications, 1997, ISBN 91-7219-045-0.

No 512 Anna Moberg: Närhet och distans - Studier av kommunikationsmönster i satellitkontor och flexibla kontor, 1997, ISBN 91-7219-119-8.

No 520 Mikael Ronström: Design and Modelling of a Parallel Data Server for Telecom Applications, 1998, ISBN 91-7219-169-4.

No 522 Niclas Ohlsson: Towards Effective Fault Prevention - An Empirical Study in Software Engineering, 1998, ISBN 91-7219-176-7.

No 526 Joachim Karlsson: A Systematic Approach for Prioritizing Software Requirements, 1998, ISBN 917219-184-8.

No 530 Henrik Nilsson: Declarative Debugging for Lazy Functional Languages, 1998, ISBN 91-7219-197-X.

No 555 Jonas Hallberg: Timing Issues in High-Level Synthesis, 1998, ISBN 91-7219-369-7.

No 561 Ling Lin: Management of 1-D Sequence Data - From Discrete to Continuous, 1999, ISBN 91-7219-402-2.

No 563 Eva L Ragnemalm: Student Modelling based on Collaborative Dialogue with a Learning Companion, 1999, ISBN 91-7219-412-X.

No 567 Jörgen Lindström: Does Distance matter? On geographical dispersion in organisations, 1999, ISBN 917219-439-1.

No 582 Vanja Josifovski: Design, Implementation and Evaluation of a Distributed Mediator System for Data Integration, 1999, ISBN 91-7219-482-0.

No 589 Rita Kovordányi: Modeling and Simulating Inhibitory Mechanisms in Mental Image Reinterpretation - Towards Cooperative HumanComputer Creativity, 1999, ISBN 91-7219-506-1.

No 592 Mikael Ericsson: Supporting the Use of Design Knowledge - An Assessment of Commenting Agents, 1999, ISBN 91-7219-532-0.

No 593 Lars Karlsson: Actions, Interactions and Narratives, 1999, ISBN 91-7219-534-7.

No 594 C. G. Mikael Johansson: Social and Organizational Aspects of Requirements Engineering Methods - A practice-oriented approach, 1999, ISBN 91-7219-541$X$.

No 595 Jörgen Hansson: Value-Driven Multi-Class Overload Management in Real-Time Database Systems, 1999, ISBN 91-7219-542-8.

No 596 Niklas Hallberg: Incorporating User Values in the Design of Information Systems and Services in the Public Sector: A Methods Approach, 1999, ISBN 917219-543-6.

No 597 Vivian Vimarlund: An Economic Perspective on the Analysis of Impacts of Information Technology: From Case Studies in Health-Care towards General Models and Theories, 1999, ISBN 91-7219-544-4.

No 598 Johan Jenvald: Methods and Tools in ComputerSupported Taskforce Training, 1999, ISBN 91-7219547-9.

No 607 Magnus Merkel: Understanding and enhancing translation by parallel text processing, 1999, ISBN 917219-614-9.

No 611 Silvia Coradeschi: Anchoring symbols to sensory data, 1999, ISBN 91-7219-623-8.

No 613 Man Lin: Analysis and Synthesis of Reactive Systems: A Generic Layered Architecture Perspective, 1999, ISBN 91-7219-630-0. 
No 618 Jimmy Tjäder: Systemimplementering i praktiken En studie av logiker i fyra projekt, 1999, ISBN 917219-657-2.

No 627 Vadim Engelson: Tools for Design, Interactive Simulation, and Visualization of Object-Oriented Models in Scientific Computing, 2000, ISBN 91-7219709-9.

No 637 Esa Falkenroth: Database Technology for Control and Simulation, 2000, ISBN 91-7219-766-8.

No 639 Per-Arne Persson: Bringing Power and Knowledge Together: Information Systems Design for Autonomy and Control in Command Work, 2000, ISBN 91-7219796-X.

No 660 Erik Larsson: An Integrated System-Level Design for Testability Methodology, 2000, ISBN 91-7219-890-7.

No 688 Marcus Bjäreland: Model-based Execution Monitoring, 2001, ISBN 91-7373-016-5.

No 689 Joakim Gustafsson: Extending Temporal Action Logic, 2001, ISBN 91-7373-017-3.

No 720 Carl-Johan Petri: Organizational Information Provision - Managing Mandatory and Discretionary Use of Information Technology, 2001, ISBN 91-7373-1269.

No 724 Paul Scerri: Designing Agents for Systems with Adjustable Autonomy, 2001, ISBN 91-7373-207-9.

No 725 Tim Heyer: Semantic Inspection of Software Artifacts: From Theory to Practice, 2001, ISBN 917373-208-7.

No 726 Pär Carlshamre: A Usability Perspective on Requirements Engineering - From Methodology to Product Development, 2001, ISBN 91-7373-212-5.

No 732 Juha Takkinen: From Information Management to Task Management in Electronic Mail, 2002, ISBN 917373-258-3.

No 745 Johan Åberg: Live Help Systems: An Approach to Intelligent Help for Web Information Systems, 2002, ISBN 91-7373-311-3.

No 746 Rego Granlund: Monitoring Distributed Teamwork Training, 2002, ISBN 91-7373-312-1.

No 757 Henrik André-Jönsson: Indexing Strategies for Time Series Data, 2002, ISBN 917373-346-6.

No 747 Anneli Hagdahl: Development of IT-supported Interorganisational Collaboration - A Case Study in the Swedish Public Sector, 2002, ISBN 91-7373-314-8.

No 749 Sofie Pilemalm: Information Technology for NonProfit Organisations - Extended Participatory Design of an Information System for Trade Union Shop Stewards, 2002, ISBN 91-7373-318-0.

No 765 Stefan Holmlid: Adapting users: Towards a theory of use quality, 2002, ISBN 91-7373-397-0.

No 771 Magnus Morin: Multimedia Representations of Distributed Tactical Operations, 2002, ISBN 91-7373-4217.

No 772 Pawel Pietrzak: A Type-Based Framework for Locating Errors in Constraint Logic Programs, 2002, ISBN 91-7373-422-5.

No 758 Erik Berglund: Library Communication Among Programmers Worldwide, 2002, ISBN 91-7373-349-0.

No 774 Choong-ho Yi: Modelling Object-Oriented Dynamic Systems Using a Logic-Based Framework, 2002, ISBN 91-7373-424-1.

No 779 Mathias Broxvall: A Study in the Computational Complexity of Temporal Reasoning, 2002, ISBN 917373-440-3.
No 793 Asmus Pandikow: A Generic Principle for Enabling Interoperability of Structured and Object-Oriented Analysis and Design Tools, 2002, ISBN 91-7373-479-9.

No 785 Lars Hult: Publika Informationstjänster. En studie av den Internetbaserade encyklopedins bruksegenskaper, 2003, ISBN 91-7373-461-6.

No 800 Lars Taxén: A Framework for the Coordination of Complex Systems' Development, 2003, ISBN 917373-604-X.

No 808 Klas Gäre: Tre perspektiv på förväntningar och förändringar i samband med införande av informationssystem, 2003, ISBN 91-7373-618-X.

No 821 Mikael Kindborg: Concurrent Comics programming of social agents by children, 2003, ISBN 91-7373-651-1.

No 823 Christina Ölvingson: On Development of Information Systems with GIS Functionality in Public Health Informatics: A Requirements Engineering Approach, 2003, ISBN 91-7373-656-2.

No 828 Tobias Ritzau: Memory Efficient Hard Real-Time Garbage Collection, 2003, ISBN 91-7373-666-X.

No 833 Paul Pop: Analysis and Synthesis of Communication-Intensive Heterogeneous Real-Time Systems, 2003, ISBN 91-7373-683-X.

No 852 Johan Moe: Observing the Dynamic Behaviour of Large Distributed Systems to Improve Development and Testing - An Empirical Study in Software Engineering, 2003, ISBN 91-7373-779-8.

No 867 Erik Herzog: An Approach to Systems Engineering Tool Data Representation and Exchange, 2004, ISBN 91-7373-929-4.

No 872 Aseel Berglund: Augmenting the Remote Control: Studies in Complex Information Navigation for Digital TV, 2004, ISBN 91-7373-940-5.

No 869 Jo Skåmedal: Telecommuting's Implications on Travel and Travel Patterns, 2004, ISBN 91-7373-935-9.

No 870 Linda Askenäs: The Roles of IT - Studies of Organising when Implementing and Using Enterprise Systems, 2004, ISBN 91-7373-936-7.

No 874 Annika Flycht-Eriksson: Design and Use of Ontologies in Information-Providing Dialogue Systems, 2004, ISBN 91-7373-947-2.

No 873 Peter Bunus: Debugging Techniques for EquationBased Languages, 2004, ISBN 91-7373-941-3.

No 876 Jonas Mellin: Resource-Predictable and Efficient Monitoring of Events, 2004, ISBN 91-7373-956-1.

No 883 Magnus Bång: Computing at the Speed of Paper: Ubiquitous Computing Environments for Healthcare Professionals, 2004, ISBN 91-7373-971-5.

No 882 Robert Eklund: Disfluency in Swedish humanhuman and human-machine travel booking dialogues, 2004, ISBN 91-7373-966-9.

No 887 Anders Lindström: English and other Foreign Linguistic Elements in Spoken Swedish. Studies of Productive Processes and their Modelling using Finite-State Tools, 2004, ISBN 91-7373-981-2.

No 889 Zhiping Wang: Capacity-Constrained Production-inventory systems - Modelling and Analysis in both a traditional and an e-business context, 2004, ISBN 9185295-08-6.

No 893 Pernilla Qvarfordt: Eyes on Multimodal Interaction, 2004, ISBN 91-85295-30-2.

No 910 Magnus Kald: In the Borderland between Strategy and Management Control - Theoretical Framework and Empirical Evidence, 2004, ISBN 91-85295-82-5. 
No 918 Jonas Lundberg: Shaping Electronic News: Genre Perspectives on Interaction Design, 2004, ISBN 9185297-14-3.

No 900 Mattias Arvola: Shades of use: The dynamics of interaction design for sociable use, 2004, ISBN 9185295-42-6.

No 920 Luis Alejandro Cortés: Verification and Scheduling Techniques for Real-Time Embedded Systems, 2004, ISBN 91-85297-21-6.

No 929 Diana Szentivanyi: Performance Studies of FaultTolerant Middleware, 2005, ISBN 91-85297-58-5.

No 933 Mikael Cäker: Management Accounting as Constructing and Opposing Customer Focus: Three Case Studies on Management Accounting and Customer Relations, 2005, ISBN 91-85297-64-X.

No 937 Jonas Kvarnström: TALplanner and Other Extensions to Temporal Action Logic, 2005, ISBN 9185297-75-5.

No 938 Bourhane Kadmiry: Fuzzy Gain-Scheduled Visual Servoing for Unmanned Helicopter, 2005, ISBN 9185297-76-3.

No 945 Gert Jervan: Hybrid Built-In Self-Test and Test Generation Techniques for Digital Systems, 2005, ISBN 91-85297-97-6.

No 946 Anders Arpteg: Intelligent Semi-Structured Information Extraction, 2005, ISBN 91-85297-98-4.

No 947 Ola Angelsmark: Constructing Algorithms for Constraint Satisfaction and Related Problems - Methods and Applications, 2005, ISBN 91-85297-99-2.

No 963 Calin Curescu: Utility-based Optimisation of Resource Allocation for Wireless Networks, 2005, ISBN 91-85457-07-8

No 972 Björn Johansson: Joint Control in Dynamic Situations, 2005, ISBN 91-85457-31-0.

No 974 Dan Lawesson: An Approach to Diagnosability Analysis for Interacting Finite State Systems, 2005, ISBN 91-85457-39-6.

No 979 Claudiu Duma: Security and Trust Mechanisms for Groups in Distributed Services, 2005, ISBN 91-8545754-X.

No 983 Sorin Manolache: Analysis and Optimisation of Real-Time Systems with Stochastic Behaviour, 2005, ISBN 91-85457-60-4

No 986 Yuxiao Zhao: Standards-Based Application Integration for Business-to-Business Communications, 2005, ISBN 91-85457-66-3.

No 1004 Patrik Haslum: Admissible Heuristics for Automated Planning, 2006, ISBN 91-85497-28-2.

No 1005 Aleksandra Tešanovic: Developing Reusable and Reconfigurable Real-Time Software using Aspects and Components, 2006, ISBN 91-85497-29-0.

No 1008 David Dinka: Role, Identity and Work: Extending the design and development agenda, 2006, ISBN 9185497-42-8.

No 1009 Iakov Nakhimovski: Contributions to the Modeling and Simulation of Mechanical Systems with Detailed Contact Analysis, 2006, ISBN 91-85497-43-X.

No 1013 Wilhelm Dahllöf: Exact Algorithms for Exact Satisfiability Problems, 2006, ISBN 91-85523-97-6.

No 1016 Levon Saldamli: PDEModelica - A High-Level Language for Modeling with Partial Differential Equations, 2006, ISBN 91-85523-84-4.

No 1017 Daniel Karlsson: Verification of Component-based Embedded System Designs, 2006, ISBN 91-85523-79-8
No 1018 Ioan Chisalita: Communication and Networking Techniques for Traffic Safety Systems, 2006, ISBN 9185523-77-1.

No 1019 Tarja Susi: The Puzzle of Social Activity - The Significance of Tools in Cognition and Cooperation, 2006, ISBN 91-85523-71-2.

No 1021 Andrzej Bednarski: Integrated Optimal Code Generation for Digital Signal Processors, 2006, ISBN 9185523-69-0.

No 1022 Peter Aronsson: Automatic Parallelization of Equation-Based Simulation Programs, 2006, ISBN 9185523-68-2.

No 1030 Robert Nilsson: A Mutation-based Framework for Automated Testing of Timeliness, 2006, ISBN 9185523-35-6

No 1034 Jon Edvardsson: Techniques for Automatic Generation of Tests from Programs and Specifications, 2006, ISBN 91-85523-31-3.

No 1035 Vaida Jakoniene: Integration of Biological Data, 2006, ISBN 91-85523-28-3.

No 1045 Genevieve Gorrell: Generalized Hebbian Algorithms for Dimensionality Reduction in Natural Language Processing, 2006, ISBN 91-85643-88-2.

No 1051 Yu-Hsing Huang: Having a New Pair of Glasses Applying Systemic Accident Models on Road Safety, 2006, ISBN 91-85643-64-5.

No 1054 Åsa Hedenskog: Perceive those things which cannot be seen - A Cognitive Systems Engineering perspective on requirements management, 2006, ISBN 91-85643-57-2.

No 1061 Cécile Åberg: An Evaluation Platform for Semantic Web Technology, 2007, ISBN 91-85643-31-9.

No 1073 Mats Grindal: Handling Combinatorial Explosion in Software Testing, 2007, ISBN 978-91-85715-74-9.

No 1075 Almut Herzog: Usable Security Policies for Runtime Environments, 2007, ISBN 978-91-85715-65-7.

No 1079 Magnus Wahlström: Algorithms, measures, and upper bounds for Satisfiability and related problems, 2007, ISBN 978-91-85715-55-8.

No 1083 Jesper Andersson: Dynamic Software Architectures, 2007, ISBN 978-91-85715-46-6.

No 1086 Ulf Johansson: Obtaining Accurate and Comprehensible Data Mining Models - An Evolutionary Approach, 2007, ISBN 978-91-85715-34-3.

No 1089 Traian Pop: Analysis and Optimisation of Distributed Embedded Systems with Heterogeneous Scheduling Policies, 2007, ISBN 978-91-85715-27-5.

No 1091 Gustav Nordh: Complexity Dichotomies for CSPrelated Problems, 2007, ISBN 978-91-85715-20-6.

No 1106 Per Ola Kristensson: Discrete and Continuous Shape Writing for Text Entry and Control, 2007, ISBN 97891-85831-77-7.

No 1110 He Tan: Aligning Biomedical Ontologies, 2007, ISBN 978-91-85831-56-2.

No 1112 Jessica Lindblom: Minding the body - Interacting socially through embodied action, 2007, ISBN 978-9185831-48-7.

No 1113 Pontus Wärnestål: Dialogue Behavior Management in Conversational Recommender Systems, 2007, ISBN 978-91-85831-47-0.

No 1120 Thomas Gustafsson: Management of Real-Time Data Consistency and Transient Overloads in Embedded Systems, 2007, ISBN 978-91-85831-33-3. 
No 1127 Alexandru Andrei: Energy Efficient and Predictable Design of Real-time Embedded Systems, 2007, ISBN 978-91-85831-06-7.

No 1139 Per Wikberg: Eliciting Knowledge from Experts in Modeling of Complex Systems: Managing Variation and Interactions, 2007, ISBN 978-91-85895-66-3.

No 1143 Mehdi Amirijoo: QoS Control of Real-Time Data Services under Uncertain Workload, 2007, ISBN 97891-85895-49-6.

No 1150 Sanny Syberfeldt: Optimistic Replication with Forward Conflict Resolution in Distributed Real-Time Databases, 2007, ISBN 978-91-85895-27-4.

No 1155 Beatrice Alenljung: Envisioning a Future Decision Support System for Requirements Engineering - A Holistic and Human-centred Perspective, 2008, ISBN 978-91-85895-11-3.

No 1156 Artur Wilk: Types for XML with Application to Xcerpt, 2008, ISBN 978-91-85895-08-3.

No 1183 Adrian Pop: Integrated Model-Driven Development Environments for Equation-Based Object-Oriented Languages, 2008, ISBN 978-91-7393-895-2.

No 1185 Jörgen Skågeby: Gifting Technologies Ethnographic Studies of End-users and Social Media Sharing, 2008, ISBN 978-91-7393-892-1.

No 1187 Imad-Eldin Ali Abugessaisa: Analytical tools and information-sharing methods supporting road safety organizations, 2008, ISBN 978-91-7393-887-7.

No 1204 H. Joe Steinhauer: A Representation Scheme for Description and Reconstruction of Object Configurations Based on Qualitative Relations, 2008, ISBN 978-91-7393-823-5.

No 1222 Anders Larsson: Test Optimization for Core-based System-on-Chip, 2008, ISBN 978-91-7393-768-9.

No 1238 Andreas Borg: Processes and Models for Capacity Requirements in Telecommunication Systems, 2009, ISBN 978-91-7393-700-9.

No 1240 Fredrik Heintz: DyKnow: A Stream-Based Knowledge Processing Middleware Framework, 2009, ISBN 978-91-7393-696-5.

No 1241 Birgitta Lindström: Testability of Dynamic RealTime Systems, 2009, ISBN 978-91-7393-695-8.

No 1244 Eva Blomqvist: Semi-automatic Ontology Construction based on Patterns, 2009, ISBN 978-91-7393-683-5.

No 1249 Rogier Woltjer: Functional Modeling of Constraint Management in Aviation Safety and Command and Control, 2009, ISBN 978-91-7393-659-0.

No 1260 Gianpaolo Conte: Vision-Based Localization and Guidance for Unmanned Aerial Vehicles, 2009, ISBN 978-91-7393-603-3

No 1262 AnnMarie Ericsson: Enabling Tool Support for Formal Analysis of ECA Rules, 2009, ISBN 978-91-7393598-2.

No 1266 Jiri Trnka: Exploring Tactical Command and Control: A Role-Playing Simulation Approach, 2009, ISBN 978-91-7393-571-5.

No 1268 Bahlol Rahimi: Supporting Collaborative Work through ICT - How End-users Think of and Adopt Integrated Health Information Systems, 2009, ISBN 978-91-7393-550-0.

No 1274 Fredrik Kuivinen: Algorithms and Hardness Results for Some Valued CSPs, 2009, ISBN 978-91-7393-525-8.

No 1281 Gunnar Mathiason: Virtual Full Replication for Scalable Distributed Real-Time Databases, 2009, ISBN 978-91-7393-503-6.
No 1290 Viacheslav Izosimov: Scheduling and Optimization of Fault-Tolerant Distributed Embedded Systems, 2009, ISBN 978-91-7393-482-4.

No 1294 Johan Thapper: Aspects of a Constraint Optimisation Problem, 2010, ISBN 978-91-7393-464-0.

No 1306 Susanna Nilsson: Augmentation in the Wild: User Centered Development and Evaluation of Augmented Reality Applications, 2010, ISBN 978-917393-416-9.

No 1313 Christer Thörn: On the Quality of Feature Models, 2010, ISBN 978-91-7393-394-0.

No 1321 Zhiyuan He: Temperature Aware and DefectProbability Driven Test Scheduling for System-onChip, 2010, ISBN 978-91-7393-378-0.

No 1333 David Broman: Meta-Languages and Semantics for Equation-Based Modeling and Simulation, 2010, ISBN 978-91-7393-335-3.

No 1337 Alexander Siemers: Contributions to Modelling and Visualisation of Multibody Systems Simulations with Detailed Contact Analysis, 2010, ISBN 978-91-7393317-9.

No 1354 Mikael Asplund: Disconnected Discoveries: Availability Studies in Partitioned Networks, 2010, ISBN 978-91-7393-278-3.

No 1359 Jana Rambusch: Mind Games Extended: Understanding Gameplay as Situated Activity, 2010, ISBN 978-91-7393-252-3.

No 1373 Sonia Sangari: Head Movement Correlates to Focus Assignment in Swedish, 2011, ISBN 978-91-7393-1540.

No 1374 Jan-Erik Källhammer: Using False Alarms when Developing Automotive Active Safety Systems, 2011, ISBN 978-91-7393-153-3.

No 1375 Mattias Eriksson: Integrated Code Generation, 2011, ISBN 978-91-7393-147-2.

No 1381 Ola Leifler: Affordances and Constraints of Intelligent Decision Support for Military Command and Control - Three Case Studies of Support Systems, 2011, ISBN 978-91-7393-133-5.

No 1386 Soheil Samii: Quality-Driven Synthesis and Optimization of Embedded Control Systems, 2011, ISBN 978-91-7393-102-1.

No 1419 Erik Kuiper: Geographic Routing in Intermittentlyconnected Mobile Ad Hoc Networks: Algorithms and Performance Models, 2012, ISBN 978-91-7519981-8.

No 1451 Sara Stymne: Text Harmonization Strategies for Phrase-Based Statistical Machine Translation, 2012, ISBN 978-91-7519-887-3.

No 1455 Alberto Montebelli: Modeling the Role of Energy Management in Embodied Cognition, 2012, ISBN 978-91-7519-882-8.

No 1465 Mohammad Saifullah: Biologically-Based Interactive Neural Network Models for Visual Attention and Object Recognition, 2012, ISBN 978-91-7519-838-5.

No 1490 Tomas Bengtsson: Testing and Logic Optimization Techniques for Systems on Chip, 2012, ISBN 978-917519-742-5.

No 1481 David Byers: Improving Software Security by Preventing Known Vulnerabilities, 2012, ISBN 97891-7519-784-5.

No 1496 Tommy Färnqvist: Exploiting Structure in CSPrelated Problems, 2013, ISBN 978-91-7519-711-1. 
No 1503 John Wilander: Contributions to Specification, Implementation, and Execution of Secure Software, 2013, ISBN 978-91-7519-681-7.

No 1506 Magnus Ingmarsson: Creating and Enabling the Useful Service Discovery Experience, 2013, ISBN 97891-7519-662-6.

No 1547 Wladimir Schamai: Model-Based Verification of Dynamic System Behavior against Requirements: Method, Language, and Tool, 2013, ISBN 978-917519-505-6.

No 1551 Henrik Svensson: Simulations, 2013, ISBN 978-917519-491-2.

No 1559 Sergiu Rafiliu: Stability of Adaptive Distributed Real-Time Systems with Dynamic Resource Management, 2013, ISBN 978-91-7519-471-4.

No 1581 Usman Dastgeer: Performance-aware Component Composition for GPU-based Systems, 2014, ISBN 978-91-7519-383-0.

No 1602 Cai Li: Reinforcement Learning of Locomotion based on Central Pattern Generators, 2014, ISBN 978-917519-313-7.

No 1652 Roland Samlaus: An Integrated Development Environment with Enhanced Domain-Specific Interactive Model Validation, 2015, ISBN 978-917519-090-7.

No 1663 Hannes Uppman: On Some Combinatorial Optimization Problems: Algorithms and Complexity, 2015, ISBN 978-91-7519-072-3.

No 1664 Martin Sjölund: Tools and Methods for Analysis, Debugging, and Performance Improvement of Equation-Based Models, 2015, ISBN 978-91-7519-071-6.

No 1666 Kristian Stavåker: Contributions to Simulation of Modelica Models on Data-Parallel Multi-Core Architectures, 2015, ISBN 978-91-7519-068-6.

No 1680 Adrian Lifa: Hardware/Software Codesign of Embedded Systems with Reconfigurable and Heterogeneous Platforms, 2015, ISBN 978-91-7519-0402.

No 1685 Bogdan Tanasa: Timing Analysis of Distributed Embedded Systems with Stochastic Workload and Reliability Constraints, 2015, ISBN 978-91-7519-022-8.

No 1691 Håkan Warnquist: Troubleshooting Trucks Automated Planning and Diagnosis, 2015, ISBN 97891-7685-993-3.

No 1702 Nima Aghaee: Thermal Issues in Testing of Advanced Systems on Chip, 2015, ISBN 978-91-7685949-0.

No 1715 Maria Vasilevskaya: Security in Embedded Systems: A Model-Based Approach with Risk Metrics, 2015, ISBN 978-91-7685-917-9.

No 1729 Ke Jiang: Security-Driven Design of Real-Time Embedded System, 2016, ISBN 978-91-7685-884-4.

No 1733 Victor Lagerkvist: Strong Partial Clones and the Complexity of Constraint Satisfaction Problems: Limitations and Applications, 2016, ISBN 978-91-7685856-1.

No 1734 Chandan Roy: An Informed System Development Approach to Tropical Cyclone Track and Intensity Forecasting, 2016, ISBN 978-91-7685-854-7.

No 1746 Amir Aminifar: Analysis, Design, and Optimization of Embedded Control Systems, 2016, ISBN 978-917685-826-4.

No 1747 Ekhiotz Vergara: Energy Modelling and Fairness for Efficient Mobile Communication, 2016, ISBN 978-91-7685-822-6.
No 1748 Dag Sonntag: Chain Graphs - Interpretations, Expressiveness and Learning Algorithms, 2016, ISBN 978-91-7685-818-9.

No 1768 Anna Vapen: Web Authentication using ThirdParties in Untrusted Environments, 2016, ISBN 978-91-7685-753-3.

No 1778 Magnus Jandinger: On a Need to Know Basis: A Conceptual and Methodological Framework for Modelling and Analysis of Information Demand in an Enterprise Context, 2016, ISBN 978-91-7685-713-7.

No 1798 Rahul Hiran: Collaborative Network Security: Targeting Wide-area Routing and Edgenetwork Attacks, 2016, ISBN 978-91-7685-662-8.

No 1813 Nicolas Melot: Algorithms and Framework for Energy Efficient Parallel Stream Computing on Many-Core Architectures, 2016, ISBN 978-91-7685623-9.

No 1823 Amy Rankin: Making Sense of Adaptations: Resilience in High-Risk Work, 2017, ISBN 978-917685-596-6.

No 1831 Lisa Malmberg: Building Design Capability in the Public Sector: Expanding the Horizons of Development, 2017, ISBN 978-91-7685-585-0.

No 1851 Marcus Bendtsen: Gated Bayesian Networks, 2017, ISBN 978-91-7685-525-6.

No 1852 Zlatan Dragisic: Completion of Ontologies and Ontology Networks, 2017, ISBN 978-91-7685-522-5.

No 1854 Meysam Aghighi: Computational Complexity of some Optimization Problems in Planning, 2017, ISBN 978-91-7685-519-5.

No 1863 Simon Ståhlberg: Methods for Detecting Unsolvable Planning Instances using Variable Projection, 2017, ISBN 978-91-7685-498-3.

No 1879 Karl Hammar: Content Ontology Design Patterns: Qualities, Methods, and Tools, 2017, ISBN 978-91-7685-454-9.

No 1887 Ivan Ukhov: System-Level Analysis and Design under Uncertainty, 2017, ISBN 978-91-7685-426-6.

No 1891 Valentina Ivanova: Fostering User Involvement in Ontology Alignment and Alignment Evaluation, 2017, ISBN 978-91-7685-403-7.

No 1902 Vengatanathan Krishnamoorthi: Efficient HTTPbased Adaptive Streaming of Linear and Interactive Videos, 2018, ISBN 978-91-7685-371-9.

No 1903 Lu Li: Programming Abstractions and Optimization Techniques for GPU-based Heterogeneous Systems, 2018, ISBN 978-91-7685$370-2$.

No 1913 Jonas Rybing: Studying Simulations with Distributed Cognition, 2018, ISBN 978-91-7685-3481.

No 1936 Leif Jonsson: Machine Learning-Based Bug Handling in Large-Scale Software Development, 2018, ISBN 978-91-7685-306-1.

No 1964 Arian Maghazeh: System-Level Design of GPUBased Embedded Systems, 2018, ISBN 978-91-7685175-3. 
No 1967 Mahder Gebremedhin: Automatic and Explicit Parallelization Approaches for Equation Based Mathematical Modeling and Simulation, 2019, ISBN 978-91-7685-163-0.

\section{Linköping Studies in Arts and Science}

No 504 Ing-Marie Jonsson: Social and Emotional Characteristics of Speech-based In-Vehicle Information Systems: Impact on Attitude and Driving Behaviour, 2009, ISBN 978-91-7393-478-7.

No 586 Fabian Segelström: Stakeholder Engagement for Service Design: How service designers identify and communicate insights, 2013, ISBN 978-91-7519-554-4.

No 618 Johan Blomkvist: Representing Future Situations of Service: Prototyping in Service Design, 2014, ISBN 978-91-7519-343-4.

No 620 Marcus Mast: Human-Robot Interaction for SemiAutonomous Assistive Robots, 2014, ISBN 978-917519-319-9.

No677 Peter Berggren: Assessing Shared Strategic Understanding, 2016, ISBN 978-91-7685-786-1.

No 695 Mattias Forsblad: Distributed cognition in home environments: The prospective memory and cognitive practices of older adults, 2016, ISBN 97891-7685-686-4

\section{Linköping Studies in Statistics}

No 9 Davood Shahsavani: Computer Experiments Designed to Explore and Approximate Complex Deterministic Models, 2008, ISBN 978-91-7393-976-8.

No 10 Karl Wahlin: Roadmap for Trend Detection and Assessment of Data Quality, 2008, ISBN 978-91-7393792-4.

No 11 Oleg Sysoev: Monotonic regression for large multivariate datasets, 2010, ISBN 978-91-7393-412-1.

No 13 Agné Burauskaite-Harju: Characterizing Temporal Change and Inter-Site Correlations in Daily and Subdaily Precipitation Extremes, 2011, ISBN 978-91-7393110-6.

No 14 Måns Magnusson: Scalable and Efficient Probabilistic Topic Model Inference for Textual Data, 2018, ISBN 978-91-7685-288-0.

\section{Linköping Studies in Information Science}

No 1 Karin Axelsson: Metodisk systemstrukturering- att skapa samstämmighet mellan informationssystemarkitektur och verksamhet, 1998. ISBN 9172-19-296-8.

No 2 Stefan Cronholm: Metodverktyg och användbarhet en studie av datorstödd metodbaserad systemutveckling, 1998, ISBN 9172-19-299-2.

No 3 Anders Avdic: Användare och utvecklare - om anveckling med kalkylprogram, 1999. ISBN 91-7219606-8

No 4 Owen Eriksson: Kommunikationskvalitet hos informationssystem och affärsprocesser, 2000, ISBN 917219-811-7.

No 5 Mikael Lind: Från system till process - kriterier för processbestämning vid verksamhetsanalys, 2001, ISBN 91-7373-067-X.

No 6 Ulf Melin: Koordination och informationssystem i företag och nätverk, 2002, ISBN 91-7373-278-8.

No 7 Pär J. Ågerfalk: Information Systems Actability - Understanding Information Technology as a Tool for Business Action and Communication, 2003, ISBN 917373-628-7.
No 8 Ulf Seigerroth: Att förstå och förändra systemutvecklingsverksamheter - en taxonomi för metautveckling, 2003, ISBN 91-7373-736-4.

No 9 Karin Hedström: Spår av datoriseringens värden Effekter av IT i äldreomsorg, 2004, ISBN 91-7373-9634.

No 10 Ewa Braf: Knowledge Demanded for Action Studies on Knowledge Mediation in Organisations, 2004, ISBN 91-85295-47-7.

No 11 Fredrik Karlsson: Method Configuration method and computerized tool support, 2005, ISBN 91-8529748-8.

No 12 Malin Nordström: Styrbar systemförvaltning - Att organisera systemförvaltningsverksamhet med hjälp av effektiva förvaltningsobjekt, 2005, ISBN 91-8529760-7.

No 13 Stefan Holgersson: Yrke: POLIS - Yrkeskunskap, motivation, IT-system och andra förutsättningar för polisarbete, 2005, ISBN 91-85299-43-X.

No 14 Benneth Christiansson, Marie-Therese Christiansson: Mötet mellan process och komponent - mot ett ramverk för en verksamhetsnära kravspecifikation vid anskaffning av komponentbaserade informationssystem, 2006, ISBN 91-8564322-X. 


\section{FACULTY OF SCIENCE AND ENGINEERING}

Linköping Studies in Science and Technology, Dissertation No. 1967, 2018

Department of Computer and Information Science

Linköping University

SE-581 83 Linköping, Sweden

www.liu.se 